\title{
UAB
}

Universitat Autònoma de Barcelona

\section{Volume Resistive Switching in metallic perovskite oxides driven by the Metal-Insulator Transition}

\author{
Juan Carlos Gonzalez Rosillo
}

ADVERTIMENT. L'accés als continguts d'aquesta tesi queda condicionat a l'acceptació de les condicions d'ús establertes per la següent llicència Creative Commons:

(c) (i) $\odot$ http://cat.creativecommons.org/?page_id=184

ADVERTENCIA. El acceso a los contenidos de esta tesis queda condicionado a la aceptación de las condiciones de uso establecidas por la siguiente licencia Creative Commons: (c) (i) @ $\Theta$ http://es.creativecommons.org/blog/licencias/

WARNING. The access to the contents of this doctoral thesis it is limited to the acceptance of the use conditions set by the following Creative Commons license: (c) (i) () $\Theta$ https://creativecommons.org/licenses/?lang=en 
UAB

Universitat Autònoma

de Barcelona

\title{
Volume Resistive Switching in metallic perovskite oxides driven by the Metal-Insulator Transition
}

\section{Juan Carlos Gonzalez Rosillo}

\author{
Tesi Doctoral \\ Departament de Física, Facultat de Ciències \\ Programa de Doctorat en Ciència de Materials \\ Universitat Autònoma de Barcelona
}

Supervisors: Prof. Teresa Puig Molina i Dr. Anna Palau Masoliver Tutor: Prof. Àlvar Sanchez Moreno Institut de Ciència de Materials de Barcelona (ICMAB-CSIC) 



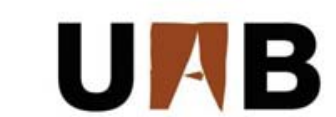

Universitat Autònoma de Barcelona
MICMAB

INSTITUT DE CIÈNCIA DE MATERIALS DE BARCELONA

EXCELENCIA SEVERO OCHOA

Memòria presentada per aspirar al Grau de Doctor per

Juan Carlos Gonzalez-Rosillo

Vist i plau

Teresa Puig Molina

(directora)
Anna Palau

Masoliver

(directora)
Àlvar Sanchez

Moreno

(tutor) 



\section{UAB \\ MICMAB \\ Universitat Autònoma \\ INSTITUT DE CIÈNCIA DE MATERIALS DE BARCELONA \\ de Barcelona \\ EXCELENCIA \\ SEVERO

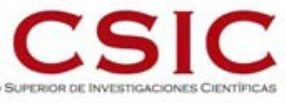

La Prof. Teresa Puig Molina, profesora de investigación a l'Institut de Ciència de Materials de Barcelona (ICMAB), pertenyent al Consejo Superior de Investigaciones Científicas,

i la Dr. Anna Palau Masoliver, científica titular a l'Institut de Ciència de Materials de Barcelona (ICMAB), pertenyent al Consejo Superior de Investigaciones Científicas,

i el Prof. Àlvar Sanchez Moreno, catedràtic d'universitat numerari del Departament de Físic de la Universitat Autònoma de Barcelona,

\section{CERTIFIQUEN:}

Que Juan Carlos Gonzalez Rosillo ha realitzat sota la seva direcció el treball d'investigació que s'exposa a la memòria titulada "Volume Resistive Switching in metallic perovskite oxides driven by the Metal-Insulator Transition" per optar al grau de Doctor per la Universitat Autónoma de Barcelona.

Que el disseny dels experiments, síntesi de mostres, llur caracteritzatió, l'anàlisi dels resultats i la redacció d'aquesta memòria són fruit del treball d'investigació realitzat per Juan Carlos Gonzalez Rosillo.

I perqué així consti, signen el present certificat,

Teresa Puig Molina Anna Palau Masoliver Àlvar Sanchez Moreno 

"Muchos años después, frente al pelotón de fusilamiento, el coronel Aureliano Buendía había de recordar aquella tarde remota en que su padre lo llevó a conocer el hielo."

Gabriel García Marquez, 100 años de soledad 



\section{Abstract}

Strongly correlated perovskite oxides are a class of materials with fascinating intrinsic physical functionalities due to the interplay of charge, spin, orbital ordering and lattice effects. The exotic phenomena arising from these competing degrees of freedom include superconductivity, ferromagnetism, ferroelectricity and metalinsulator transitions, among others. The use of these exotic phenomena in a new generation of devices with new and enhanced functionalities is continuing inspiring the research community.

In this sense, Resistive-Random Access Memories (RRAM) are one of the most promising candidates to win the race towards the universal memory of the future, which could overcome the limitations of actual technologies (Flash and DynamicRAM), due to their excellent properties in terms of scalability, endurance, retention and switching speeds. They are based on the Resistive Switching effect (RS), where the application of an electric field produces a reversible, non-volatile change in the resistance between two or more resistive states. This phenomenon has been observed in a large variety of oxide materials, where the motion of oxygen is widely accepted to play a key role in their outstanding properties. However, the exact mechanism governing this effect is material-dependent and for some of them it is still far to be understood. This lack of understanding is actually one of the main bottlenecks preventing the widespread use of this technology.

In this thesis, we present a novel Resistive Switching mechanism based on the Metal-Insulator Transition (MIT) in metallic perovskite oxides with strong electronelectron interaction. We analyse the RS behaviour of three different families of metallic perovskites: $\mathrm{La}_{1-x} \mathrm{Sr}_{x} \mathrm{MnO}_{3}, \mathrm{YBa}_{2} \mathrm{Cu}_{3} \mathrm{O}_{7-\delta}$ and $\mathrm{ReNiO}_{3}$ and demonstrate that the MIT of these mixed electronic-ionic conductors can be tuned upon the application of an electric field, being able to transform the entire bulk volume. This volume RS is different in nature from interfacial or filamentary type and opens new possibilities of robust device design.

Thorough nanoscale electrical characterization of the RS effect in these systems has been performed by means of Conductive-Atomic Force Microscopy (C-AFM). Scanning Tunnelling Spectroscopy (STS) and temperature-dependent transport measurements were performed in the different resistive states to get insight into their electronic features. The nanoscale memristive behaviour of these systems is successfully reproduced at a micrometric scale with $\mathrm{W}$-Au tips in probe station experiments. Using this approach, atmosphere dependent measurements were undertaken, where oxygen exchange with the ambience is strongly evidenced. In addition, we present a proof-of-principle result from a 3-Terminal configuration where the RS effect is applied at the gate of the device. In the particular case of superconducting $\mathrm{YBa}_{2} \mathrm{Cu}_{3} \mathrm{O}_{7-\delta}$ films, we have studied the influence of high resistance areas, which are embedded in the material, on the superconducting transport properties enabling vortex pinning modification and paving the way towards novel reconfigurable vortex pinning sites.

We interpret the RS results of these strongly correlated systems in terms of a Mott volume transition, that we believe to be of general validity for metallic perovskite complex oxides. We have verified that strongly correlated metallic perovskite oxides are a unique class of materials very promising for RS applications due to its intrinsic MIT properties that boosts a robust volumetric resistive switching effect. This thesis settles down the framework to understand the RS effect in these strongly 
correlated pervoskites, which could eventually lead to a new generation of devices exploiting the intrinsic MIT of these systems. 


\section{Contents}

Abstract vii

Motivation $\quad$ viii

1 Introduction 3

1.1 Resistive Switching phenomena in oxides . . . . . . . . . . . 3

1.1.1 Device phenomenology . . . . . . . . . . . . . . 4

1.1.2 Classification of resistive switching mechanisms . . . . . . . 5

1.2 Metal-Insulator Transitions . . . . . . . . . . . . . . . . 6

1.2.1 Mechanisms behind the Metal-Insulator Transition . . . . . 7

1.2.2 Metal-Insulator Transition for Resistive Switching applications 8

1.3 Metallic perovskite oxides displaying MIT . . . . . . . . . . . 9

1.3.1 Lanthanum strontium manganites . . . . . . . . . . . 10

Cystral and electronic structure of $\mathrm{LSMO} \ldots \ldots \ldots$

Phase diagram of $\mathrm{La}_{1-x} \mathrm{Sr}_{x} \mathrm{MnO}_{3} \ldots \ldots \ldots \ldots$

1.3.2 Rare-earth nickelates . . . . . . . . . . . . . . . . . . . . 13

Crystal and electronic structure of $\mathrm{RENiO}_{3} \ldots \ldots \ldots$

Phase diagram of $\mathrm{RENiO}_{3} \ldots \ldots \ldots \ldots$

1.3.3 Cuprate $\mathrm{YBa}_{2} \mathrm{Cu}_{3} \mathrm{O}_{7-\delta} \ldots \ldots \ldots \ldots \ldots \ldots$

Crystal and electronic structure of $\mathrm{YBa}_{2} \mathrm{Cu}_{3} \mathrm{O}_{7-\delta} \ldots \ldots \ldots$

Superconducting properties of $\mathrm{YBa}_{2} \mathrm{Cu}_{3} \mathrm{O}_{7-\delta} \ldots \ldots \ldots$

1.3 .4 Cerium dioxide . . . . . . . . . . . . . . . . . 17

1.4 Outline of the thesis . . . . . . . . . . . . . . . . 18

$\begin{array}{llr}2 & \text { Experimental procedure } & 21\end{array}$

2.1 Sample preparation . . . . . . . . . . . . . . . . . 21

2.1.1 Substrate selection and preparation . . . . . . . . . 21

2.1.2 Growth and characterization of $\mathrm{La}_{1-x} \mathrm{Sr}_{x} \mathrm{MnO}_{3}$ thin films . . . 23

CSD route for $\mathrm{LSMO}$ growth $\ldots . \ldots . \ldots 23$

Structural characterization of LSMO thin films . . . . . . . . 25 
Morphological characterization of LSMO thin films . . . . . 26

Physical properties of LSMO thin films . . . . . . . . . 27

2.1.3 Growth and characterization of $\mathrm{RENiO}_{3}$ thin films . . . . . . 28

CSD route for RENO growth . . . . . . . . . . . . . . . . . . 29

Structural characterization of RENO thin films . . . . . . . . 29

Morphological characterization of RENO thin films . . . . . 30

Physical properties of RENO thin films . . . . . . . . . . . 31

2.1.4 Growth and characterization of $\mathrm{YBa}_{2} \mathrm{Cu}_{3} \mathrm{O}_{7-\delta}$ thin films . . . 32

PLD route for $\mathrm{YBCO}$ growth $\ldots \ldots \ldots$. . . . . . . . 32

Structural characterization of YBCO thin films . . . . . . . 33

Morphological characterization of YBCO thin films . . . . . 34

Physical properties of YBCO superconducting films . . . . . 35

2.1.5 Growth and characterization of $\mathrm{CeO}_{2} / \mathrm{LSMO}$ and $\mathrm{CeO}_{2} / \mathrm{YBCO}$ bilayer systems $\ldots \ldots \ldots \ldots . \ldots . \ldots 36$

ALD route for $\mathrm{CeO}_{2}$ growth . . . . . . . . . . . . . 37

Structural characterization of $\mathrm{CeO}_{2}$ bilayer systems . . . . . 37

Morphological characterization of $\mathrm{CeO}_{2}$ bilayer systems . . . 38

Physical properties of $\mathrm{CeO}_{2}$ bilayer systems $\ldots \ldots \ldots . . . .38$

2.1.6 Metal deposition and device fabrication . . . . . . . . . . . . 39

Photolithography . . . . . . . . . . . . . . . . . . . 39

Metal deposition and lift-off . . . . . . . . . . . . . . . . . 40

Oxide etching . . . . . . . . . . . . . . . 4 41

2.2 Characterization techniques . . . . . . . . . . . . . . 42

2.2.1 Scanning Tunnelling Microscopy . . . . . . . . . . . . . . . 42

Operation modes . . . . . . . . . . . . . . . 43

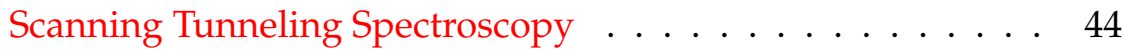

STM protocol measurement . . . . . . . . . . . . . . 45

2.2.2 Atomic Force Microscopy . . . . . . . . . . . . . . . . . 47

Operation modes . . . . . . . . . . . . . . . . . 48 48

Conductive-Atomic Force Microscope . . . . . . . . . . . . 50

Protocol for RS measurement by C-AFM . . . . . . . . . . 51

2.2.3 Probe Station measurements for RS at a micrometer scale . . . 54

2.2.4 Other characterization techniques . . . . . . . . . 55

2.3 Conclusions . . . . . . . . . . . . . . . . . . . 56 
3 Resistive Switching in LSMO thin films 59

3.1 Resistive Switching in LSMO films studied by C-AFM . . . . . . . . . 60

3.1.1 Electrical characterization by I-V sweeps . . . . . . . . . . . 60

3.1.2 Micrometric scale HRS areas induced by C-AFM . . . . . . . . 63

3.1.3 Volume Resistive Switching in LSMO thin films . . . . . . . 66

3.1.4 Dependence of Sr content on the RS properties . . . . . . . . . 69

3.2 Ex-situ characterization of LSMO thin films by STM/S . . . . . . 72

3.2.1 Sample preparation and measurement procedure . . . . . . 72 Sample preparation at ICMAB $\ldots \ldots \ldots \ldots$

Measurement procedure at University of Geneva . . . . . . 73

3.2.2 STM/S studies in the pristine state of $\mathrm{La}_{1-\mathrm{x}} \mathrm{Sr}_{\mathrm{x}} \mathrm{MnO}_{3}$ thin films 75

3.2.3 STM/S of ex-situ generated HRS regions in $\mathrm{La}_{0.8} \mathrm{Sr}_{0.2} \mathrm{MnO}_{3}$ thin films . . . . . . . . . . . . . . . 77

3.2.4 RS study in $\mathrm{La}_{1-x} \mathrm{Sr}_{x} \mathrm{MnO}_{3}$ films with different Sr content . . . 79

3.3 Electrical transport properties of HRS areas . . . . . . . . . . . 82

3.3.1 Sample preparation and measurement protocol . . . . . . 82

3.3.2 Selection of voltages to induce the HRS . . . . . . . . . 83

3.3.3 The $-8 \mathrm{~V}$ HRS case . . . . . . . . . . . . . . . . . 84

3.3.4 The -10 V HRS case . . . . . . . . . . . . . . . 85

3.3 .5 Discussion . . . . . . . . . . . . . . . . 88

3.4 Atmosphere dependence of the RS effect . . . . . . . . . . . . . 89

RS studies with microscopic tips . . . . . . . . . . . 90

3.4.1 RS at different total pressures . . . . . . . . . . . . . . 92

3.4.2 RS at different oxygen pressures . . . . . . . . . . . . 94

3.4.3 RS at different moisture conditions . . . . . . . . . . . . . 95

3.4 .4 Discussion . . . . . . . . . . . . . . . . . 96

3.5 Proof of concept of 3-Terminal device . . . . . . . . . . . 100

3.5.1 Device fabrication and operation . . . . . . . . . . 100

3.5.2 Results and discussion . . . . . . . . . . . . . . . . . . 101

3.6 Conclusions . . . . . . . . . . . . . . . . . . 104

$\begin{array}{lll}4 & \text { Resistive Swithching in other complex oxides } & 107\end{array}$

4.1 RS behaviour in YBCO thin films . . . . . . . . . . . . . . 107

4.1 .1 Introduction . . . . . . . . . . . . . . . . 107

4.1.2 RS characterization in bare YBCO thin films . . . . . . . . 107 
Electrical characterization by I-V sweeps _. . . . . . . . 108

Micrometic scale HRS areas induced by C-AFM . . . . . . . 110

Volume Resistive Switching in PLD YBCO thin films . . . . . 113

Transport studies of HRS arrays and its effect on the superconducting transition . . . . . . . . . . . . 114

4.1.3 RS characterization in $\mathrm{CeO}_{2} / \mathrm{YBCO}$ bilayer systems . . . . . 118

I-V curves and HRS maps induced by C-AFM . . . . . . . 119

Transport properties of HR arrays in bilayer systems. . . . . . 120

4.1 .4 Conclusions . . . . . . . . . . . . . . . . . . . . . . . . . 124

4.2 Resistive Switching in $\mathrm{RENiO}_{3}$ systems . . . . . . . . . . . . 126

4.2 .1 Introduction . . . . . . . . . . . . . . . . . . . 126

4.2.2 RS characterization in $\mathrm{RENiO}_{3}$ grown by CSD . . . . . . . 126

Electrical characterization by I-V sweeps . . . . . . . . 126

Microstructure of RENO thin films . . . . . . . . . . . . . . . 129

Micrometric HR induced areas . . . . . . . . . . . . . . 131

Volume Switching in RENO thin films . . . . . . . . . . . . 132

RS characterization with micrometric W-Au probes . . . . . 132

Resistive Switching mechanism in RENO systems . . . . . . . 134

4.2.3 RS characterization in $\mathrm{RENiO}_{3}$ grown by RF-Sputtering . . 134

Electrical characterization by I-V sweeps . . . . . . . . . . 135

Discussion . . . . . . . . . . . . . . . . . 136

4.2 .4 Conclusions . . . . . . . . . . . . . . . . . . . . 138

5 General conclusions $\quad 141$

$\begin{array}{ll}\text { A Experimental techniques } & \mathbf{1 4 5}\end{array}$

A.1 Field Emission Scanning Electron Microscopy . . . . . . . . . . . . . 145

A.2 X-ray diffraction . . . . . . . . . . . . . . . 146

A.3 Physical Properties Measurement System ～. . . . . . . . . . . . . 147

A.4 Superconducting Quantum Interference Device . . . . . . . . . . 147

$\begin{array}{lr}\text { Bibliography } & 149\end{array}$ 


\section{List of Abbreviations}

\begin{tabular}{|c|c|}
\hline $3-\mathrm{T}$ & Three-Terminal \\
\hline ads & Adsorbates \\
\hline AFM & Atomic Force Microscopy \\
\hline ALD & Atomic Layer Deposition \\
\hline C-AFM & Conductive-AFM \\
\hline CCDC & Current Carrier Density Collapse Model \\
\hline CC's & Coated Conductors \\
\hline $\mathrm{CeO}_{2}$ & Cerium dioxide or ceria \\
\hline CITS & Current-imaging Tunnelling Spectroscopy \\
\hline CMR & Colossal Magnetoresistance \\
\hline CSD & Chemical Solution Deposition \\
\hline DE & Double Exchange Mechanism \\
\hline DOS & Density of States \\
\hline $\mathbf{E}_{f}$ & Fermi Energy \\
\hline ECM & Electrochemical Metallization Mechanism \\
\hline FET & Field Effect Transistor \\
\hline FV & Formal Valence \\
\hline G & Conductance $G=d I / d V$ \\
\hline HRS & High Resistance State \\
\hline HTS & High Temperature Superconductors \\
\hline $\mathbf{I}_{c}$ & Critical current \\
\hline $\mathbf{I}_{s p}$ & Current set-point \\
\hline I-V or I(V) & Current-voltage curves \\
\hline $\mathbf{J}_{c}$ & Critical Current Density \\
\hline $\mathrm{J}-\mathrm{T}$ & Jahn-Teller Distortions \\
\hline LAO & Lanthanum Aluminate $\mathrm{LaAlO}_{3}$ \\
\hline LDOS & Local Density of States \\
\hline LHB & Lower Hubbard Band \\
\hline LDOS & Local Density of States \\
\hline LNO & Lanthanum Nickelate $\mathrm{LaNiO}_{3}$ \\
\hline LRS & Low Resistance State \\
\hline LSMO & Lanthanum Strontium Manganite $\left(\mathrm{La}_{1-x} \mathrm{Sr}_{x} \mathrm{MnO}_{3}\right.$ \\
\hline MIT & Metal-Insulator Transition \\
\hline $\mathbf{M n}_{F V}$ & Mn ion with oxidation state $=\mathrm{FV}$ \\
\hline NNO & Niodimium Nickelate $\mathrm{NdNiO}_{3}$ \\
\hline OM & Optical Microscopy \\
\hline $\mathbf{p}\left(\mathrm{O}_{2}\right)$ & Oxygen partial pressure \\
\hline PCM & Phase Change Materials \\
\hline PLD & Pulsed Laser Deposition \\
\hline PPMS & Physical Properties Measurement System \\
\hline $\mathbf{R}$ & Resistance \\
\hline RAM & Random Access Memory \\
\hline RDS & Rate Determining Step \\
\hline
\end{tabular}




\begin{tabular}{|c|c|}
\hline RE & Rare-Earth \\
\hline RENO & Rare-earth nickeates $\mathrm{ReNiO}_{3}$ \\
\hline RH & Relative Humidity \\
\hline RMS & Root mean square \\
\hline RPF & Ruddlesden Popper Fault \\
\hline RRAM & Resistive RAM \\
\hline RS & Resistive Switching \\
\hline SC & Superconductivity \\
\hline SEM & Scanning Electron Microscopy \\
\hline SPM & Scanning Probe Microscopy \\
\hline SQUID & Superconducting Quantum Interference Device \\
\hline STEM & Scanning Transmission Electron Microscopy \\
\hline STM & Scanning Tunnelling Microscopy \\
\hline STO & Strontium Titanate $\mathrm{SrTiO}_{3}$ \\
\hline STS & Scanning Tunnelling Spectroscopy \\
\hline $\mathbf{T}$ & Temperature \\
\hline $\mathbf{T}_{c}$ & Superconducting Transition Temperature \\
\hline $\mathbf{T}_{M} I T$ & Temperature at which the MIT occurs \\
\hline TCM & Thermochemical Mechanism \\
\hline TEM & Transmission Electron Microscopy \\
\hline TF & Tolerance Factor \\
\hline TMO & Transition Metal Oxide \\
\hline UHB & Upper Hubber Band \\
\hline UV & Ultraviolet \\
\hline$V_{\text {bias }}$ & Voltage Applied \\
\hline$V_{\max }$ & Maximum voltage \\
\hline VCM & Valence Change Mechanism \\
\hline YBCO & Yttrium Barium Copper Oxide $\mathrm{Yba}_{2} \mathrm{Cu}_{3} \mathrm{O}_{7-\delta}$ \\
\hline ZBC & Zero Bias Conductance \\
\hline
\end{tabular}




\section{Motivation}

The demand for data storage has rapidly increased in the last decades with the outbreak of the digital era. Nowadays, the memory market is dominated by two technologies: Dynamic Random access memories (DRAM) and Flash memories. However, there is a lack of an universal memory with the advantages of both technologies (fast switching and large retention and endurance) which can overcome the scalability limit that the conventional technologies present.

A lot of efforts are currently being undertaken in the research community to address this issue. In this sense, the resistive switching (RS) phenomenon is a very appealing physical effect owing to a memory behaviour with outstanding performance, beating the actual Flash and DRAM technologies.

The RS effect has been observed in a large variety of materials, including chalcogenides, binary oxides and complex oxides. In this latter class of materials, the intricate interplay of charge, spin, orbital ordering and lattice effects leads to exotic phenomena, such as ferromagnetism, ferroelectricity, superconductivity and metal-insulator transition (MIT).

These properties can be controlled upon material engineering in order to design novel devices that could fully exploit the intrinsic functional properties of the material. In particular, materials showing MIT can present a wide range of resistive states upon small variations in the charge carrier density. This is the case of three different families of metallic perovskite oxides: manganites, nickelates and cuprates.

In this thesis, we explore the RS effect in these three families at the nano- and microscales with local probe techniques. We demonstrate the link between the RS effect observed in these compounds and their MIT, thus shedding new light into the physical origin of the effect. Despite the different origin of the MIT in the three systems, we have found common features among them that allow us to generalize a model for metallic perovskite oxides with MIT.

This work is intended to depict the framework for the analysis of the RS effect in strongly correlated metallic perovskite systems and to demonstrate their promising capabilities for further device integration. We will show the unique opportunities of these materials by thoroughly studying their resistive switching properties at the nano- and microscales, mainly by scanning probe techniques. We will prove the capabilities of these materials to show an homogeneous volumetric Resistive Switching effect with excellent performances, making them highly appealing candidates for the next generation of R-RAM devices. 



\section{Chapter 1}

\section{Introduction}

The concise summary made in this chapter is addressed to provide a comprehensive overview of the resistive switching effect and the state-of-the-art knowledge on the main mechanisms responsible for it. The recent advancements in the field are also highlighted. Special emphasis lays on the metal-insulator transitions in strongly correlated oxides. A brief introduction on the basics of the materials employed in this thesis is also given.

\subsection{Resistive Switching phenomena in oxides}

Our information-based society requires handling large amounts of data and computing power. In 2007, it was estimated that more than 2 zettabytes of information were stored worldwide, and it is predicted to reach 16 ZB in 2017 [1]. Therefore, there is a huge research interest in technologies that could increase our information storage capacity in a near future. At present, two main types of memories dominates the market: Dynamic random access memories (DRAM) and Flash memories. On the one hand, DRAM are based on capacitance effects and present excellent performance (writing, erasing and reading times of less then $10 \mathrm{~ns}$ ) and large endurance ( $>10^{6}$ cycles). However, DRAM devices present poor retention times $(\sim 60 \mathrm{~ms})$ and they have to be periodically refreshed. Therefore, DRAM are volatile memories with high power-consumption in operation. On the other hand, Flash technology is based on charge trapping effects and show slower performance than DRAM (writing, erasing and reading times in the $0.1-1 \mathrm{~ms}$ range) and poor endurance ( $>10^{4}$ cycles). Advantageously, they present large retention times $(10-20 y r s)$, being thus non-volatile memories. The fast performance of DRAM makes them suitable for instructions computing, whereas Flash memories are widely used in data storage. The seek of an universal memory combining the advantages of the two aforementioned technologies, i.e., high density and speed, low power consumption and long data retention, is currently inspiring remarkable efforts in the research community and industry.

The prediction of Gordon E. Moore in 1965 in which the number of transistors doubles every two years, known as "Moore's Law" [2], has proven to be accurate up to now. However, further reduction of transistor size is limited by high dissipation and quantum mechanical effects, which degrade device properties in the current CMOS technology [3]. Therefore, other concepts have to be developed for further improvement of devices to meet the required performances. One of the strategies adopted by the community to overcome these problems is the so called "Beyond CMOS". Here, new state variables such as spin, molecular state, photons, phonons, 
nano-structures, mechanical state, resistance, quantum state (including phase) and quantized magnetic flux are investigated for their suitability in future nanoelectronic devices [4].

Different random access memories (RAM) are currently being considered as alternatives to Flash technology [5, 6], such as magnetic-RAM (MRAM) [7, 8], phase change-RAM (PCRAM) [9, 10], ferroelectric-RAM (Fe-RAM) [11, 12] or ResistiveRAM (RRAM) [13-16]. The latter case, RRAM, is considered a very promising emerging alternative for its simple architecture and excellent performances. RRAM devices are based on the so called Resistive Switching (RS) effect, where two or more reversible resistance states can be induced upon the application of an electric field due to a thermal, chemical or electronic change. The more conducting state is called the ON or Low Resistance State (LRS), whereas the less conducting state is called OFF or high resistance state (HRS). In the literature, the $\mathrm{OFF} \rightarrow \mathrm{ON}$ transition is called SET, while the $\mathrm{ON} \rightarrow \mathrm{OFF}$ process is called RESET. However, this nomenclature might induce confusion in our case as it will be shown later. Thus, in our experiments the terms HRS (or OFF state) and LRS (or ON state) will be employed and the transition (HRS $\rightarrow$ LRS or LRS $\rightarrow$ HRS) specified.

Several oxide systems present RS, including binary and complex oxides, such as $\mathrm{TiO}_{2}$ [17], $\mathrm{NiO}$ [18], $\mathrm{TaO}_{x}$ [19], $\mathrm{Pr}_{x} \mathrm{Ca}_{1-x} \mathrm{MnO}_{3}$ [20-22], or $\mathrm{SrTiO}_{3}$ [23-26]. Diverse thermochemical and electrochemical effects have been proposed as the microscopic origin of the RS for the different materials. They include the formation/rupture of conducting filaments $[17,27,28]$, modification of a Schottky barrier close to the metal/oxide interface [29, 30] and metal-insulator (MIT) Mott transition [31, 32] among others. Before classifying the different mechanisms in more detail, let us introduce the device phenomenology. For a more detailed comprehension, the reader is referred to the following reviews $[15,16,33]$.

\subsubsection{Device phenomenology}

The typical metal oxide memory RS cell is a simple metal-insulator-metal (MIM) structure as shown in figure 1.1 (a), since most of the materials studied are insulators. The switching modes of these cells can be broadly classified into two different types: unipolar and bipolar [14]. In the unipolar resistive switching (URS) the switching process depends on the amplitude of the applied voltage but not on the polarity, and usually it can symmetrically occur at both forward and reversed voltages, as depicted in figure 1.1 (b). The compliance current (CC) limits the current during operation of the device, and it is usually larger in the $\mathrm{ON} \rightarrow \mathrm{OFF}$ than in the $\mathrm{OFF} \rightarrow \mathrm{ON}$ processes. In contrast, in the bipolar resistive switching (BRS), both transitions occur only at one polarity and at a certain bias, i.e., the switching depends on the polarity and on the magnitude of the applied bias. In both cases, the electrical readout of memory state of the cell is performed at very low bias to assure that it does not affect its resistive state. A scheme is shown in figure 1.1 (c). In addition, both mechanisms have been observed even in the same material and considerable effort is undertaken to control the coexistence of both [34-37].

In some cases a larger intial voltage is needed in a fresh device to induce the resistive switching effect in the subsequent cycles. This is called the Electroforming process $[38,39]$. 
a)

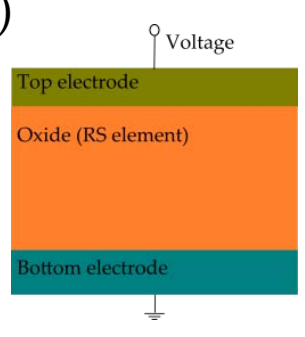

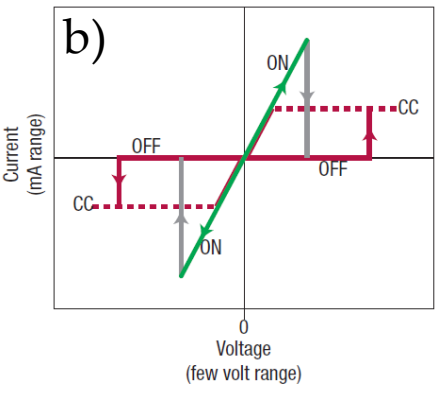

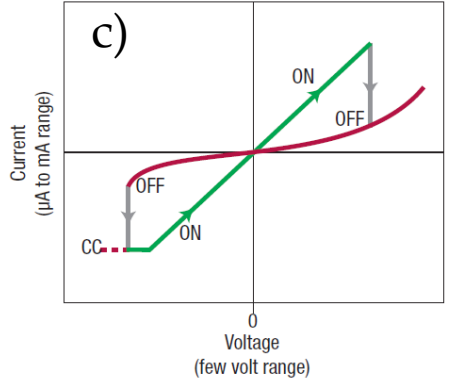

FIGURE 1.1: (a) Typical structure of a MIM cell. I-V sweeps showing the two mentioned operation modes: (b) unipolar and (c) bipolar. Adapted from [14].

\subsubsection{Classification of resistive switching mechanisms}

Several physical phenomena produce a non-volatile resistance switch that might lead to a RS memory effect. For instance, a single molecule might switch between resistance states upon a change in its molecular configuration [40] or by controlling the direction of a ferroelectric domain polarization one can tune the tunnelling current [41] across the tunnel junction. In PCM, either the Joule heating effect or heating through optical pulses is used to induce a change between a crystalline phase (LRS) and an amorphous phase (HRS) in tellurides and selenides [9, 42]. Besides them, there are three main RS mechanisms which involve chemical effects [15], i.e., the switching involves red-ox processes in the memristive devices either triggered by temperature or electrical voltage or both: the thermochemical mechanism (TCM), the electrochemical metallization mechanism (ECM) and valence change mechanism (VCM). The TCM [15, 43] occurs due to a change of the stoichiometry which varies locally the conductivity produced by current-induced increase of the temperature. It is inherently unipolar and it is associated to filamentary conduction paths. The ECM [28] is based on an electrochemically active metal electrode such as $\mathrm{Ag}$ or $\mathrm{Cu}$, whose highly mobile cations are transported through the solid electrolytes (oxides or higher chalcogenides) forming $(\mathrm{ON})$ and dissolving (OFF) metallic filaments. ECM is an inherently bipolar mechanism. For the VCM, which is mainly observed in transition metal oxides (TMO), several explanations have been proposed, depending on the material. For instance, it has been proposed that pure electronic effects might be responsible of the RS effect, such as the charge-trapping model at impurities [44], charge trapping at interface sites [45], pure electronic metal-insulator transition [46, 47] and polaron ordering [48]. However, in most of the TMO it is widely accepted that the valence change is mainly triggered by migration of oxygen anions (usually described as oxygen vacancies), much more mobile than the cations [15]. VCM is also inherently bipolar, since the polarity determines the reduction or oxidation of the material.

In addition, there are two different scenarios according to the geometrical localization of the switching event: the filamentary switching scenario (figure 1.2 (a) and the area distributed switching scenario (figure 1.2 (b) [15, 49]. In the case of filamentary conduction, oxygen vacancies (or metallic cation species in the ECM cells) organize into filaments connecting the two electrodes. It can be associated both to unipolar (TCM) or to bipolar (ECM and VCM) switching. In the case of interface conduction, oxygen vacancies distribute along a depleted interface which 
ultimately controls the conductivity of the device. This mechanism is usually associated to a bipolar behaviour.
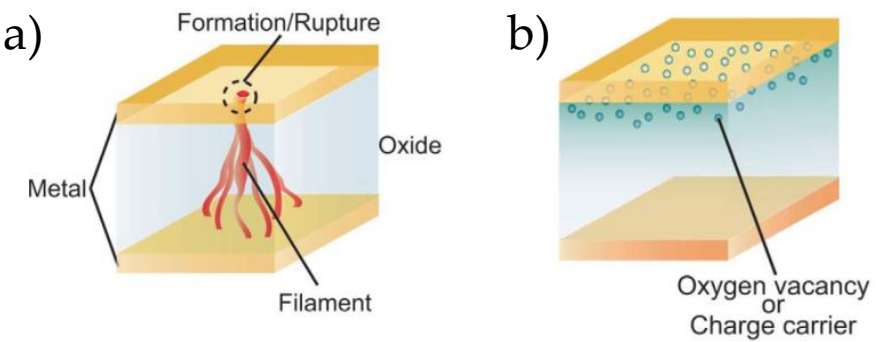

FIGURE 1.2: Geometrical localization of the switching event. (a) Filamentary conducting path and (b) interface-type conducting path. Adapted from [49].

In this thesis, we present a VCM mechanism based on the metal-insulator transition (MIT) of three different mixed-valence-mixed-conductors metallic perovskites, $\mathrm{La}_{1-x} \mathrm{Sr}_{x} \mathrm{MnO}_{3}, \mathrm{YBa}_{2} \mathrm{Cu}_{3} \mathrm{O}_{7-\delta}$ and $\mathrm{ReNiO}_{3}$. In the following chapters, we show strong evidences supporting an homogeneous switching even through the full thickness of the film. The MIT might be then induced in a volumetric and homogeneous way upon the application of an intense electric field. We thoroughly explored and extend earlier studies in the group [50,51], where a volume resistive switching was suggested for the first time in $\mathrm{La}_{0.7} \mathrm{Sr}_{0.3} \mathrm{MnO}_{3}$ thin films. Before presenting the metallic perovskites studied in this thesis, a brief overview of metalinsulator transitions is given.

\subsection{Metal-Insulator Transitions}

The metal-insulator transition (MIT) is widely observed in a large variety of materials, specifically in strongly correlated transition metal oxides (TMO). It produces an intrinsic change in the material from an insulator to a metallic state and it can be triggered by different factors, such as pressure, temperature or electric field, among others. Usually, the MIT is accompanied by colossal resistivity changes, even over several orders of magnitude. In this section, a brief overview of MIT mechanisms is presented. Deep theoretical and experimental reviews can be found elsewhere [52-57].

In the single electron band picture, the electronic structure of a solid is described as free electrons moving in an effective periodic potential forced by ions. This periodic potential produces a discontinuity in the electron dispersion curves at the boundaries of the Brillouin zone, thus creating energy bands and gaps in the momentum space. Metals present a partially filled band whereas the electron bands of insulators are fully occupied at $T=0 K$. This simple view explains exceptionally well the physical properties of many materials. However, it ignores disorder-electron interaction, phonon-electron interaction and electron-electron interaction or treats them as perturbations. Therefore, the deficiency of the simple single electron band picture is remarkable in some scenarios where these interactions become relevant. 


\subsubsection{Mechanisms behind the Metal-Insulator Transition}

Metal-insulator transitions can be categorized based on the dominant interaction driving the material into the insulating phase: disorder-electron interaction (Anderson localization), lattice-electron interaction (Peierls transition) and/or electronelectron interaction (Mott transition).

The Anderson localization [58] is produced by lattice disorder such as impurities and vacancies. Conducting electrons are eventually scattered by defects, thus decreasing their mean free path and consequently, the material conductivity. Experimentally, Anderson localization has been reported in various highly doped semiconductors, such as Sb:Ge[59] and Si:P [60, 61]. The Peierls MIT [62-64] occurs from an electron-phonon (electron- lattice) interaction. Generally, this transition arises from a structural change in the lattice of the material. This lattice deformation modifies the periodic ionic potential in the system. Thus, a change in the band structure occurs and hence the MIT is achieved. Experimentally, Peierls transition has been observed in highly anisotropic materials and quasi-one-dimensional band structures, such as $\mathrm{K}_{0.3} \mathrm{MoO}_{3}$ [65] and $\mathrm{NbS}_{3}$ [64]. In the Mott transition [52, 53, 66] a critical carrier density $n_{c}$ is proposed in such a way that, if the carrier density in the material exceeds the critical value $n_{c}$, the system is driven to the insulating state due to the strong electron-electron interaction which localizes the charge carriers. This effectively splits the original transition metal $3 \mathrm{~d}$ band into an upper and a lower Hubbard band [67] (UHB and LHB, respectively), as seen in figure 1.3. The magnitude of the gap is denoted as $\mathrm{U}$ whereas $\mathrm{W}$ corresponds to the bandwidth. By tuning the relative magnitude of $U$ and $\mathrm{W}$, a MIT might be achieved.

a)

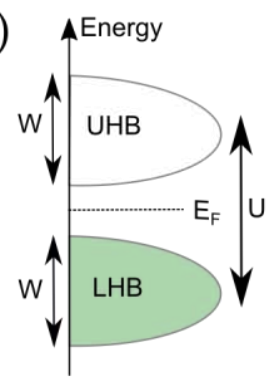

b)

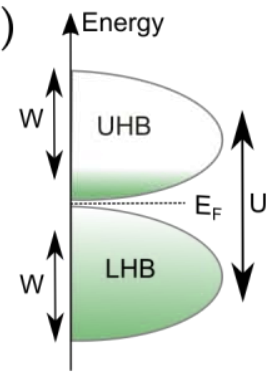

FIGURE 1.3: (a) Schematic of the splitting of the transition metal 3d band into the Upper Hubbard Band and Lower Hubbard Band. (b) Induction of the MIT by changing the $\mathrm{U} / \mathrm{W}$ ratio.

Two different scenarios arise depending on the relevance of the oxygen $2 p$ band on electron (hole) conduction: a) the oxygen $2 p$ band falls below the lower Hubbard band (called Mott-Hubbard insulators, main gap called $U$ ) and $b$ ) the oxygen $2 p$ band falls in between the two Hubbard bands (called Charge-transfer insulators, main gap called $\Delta$ ) (see reference [57] and references therein), as shown in figure 1.4. In the former case, conducting electrons/holes hop from transition metal to transition metal, while in the latter the conduction takes place by electron/hole hopping from transition metal to oxygen to transition metal. In both cases, a metal insulator transition (MIT) is induced and can be tuned either by controlling the bandwidth (e.g., by changing interatomic distances (cation substitution [68], pressure [69], strain [70-72]) or temperature) or by controlling the band-filling (chemical doping [56, 73], application of an electric field $[50,56])$. 
a)

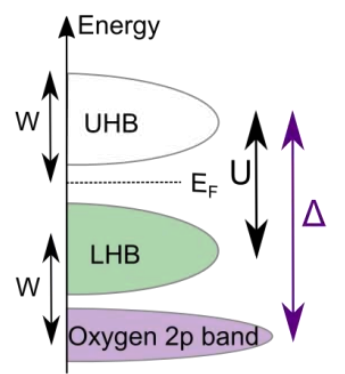

b)

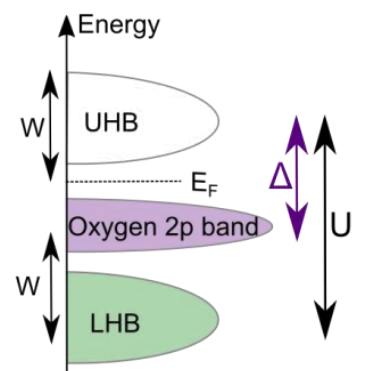

FIGURE 1.4: Mott-Hubbard insulator where the oxygen p-band falls below the lower Hubbard band (a) and charge-transfer insulator where oxygen $\mathrm{p}$-band is in between the Hubbard bands (b).

MIT in cuprates [74, 75] and manganites [76] is mostly controlled by the bandfilling mechanism whereas in rare-earth nickelates a bandwidth-control of the MIT $[68,72,77]$ can be easily achieved . Figure 1.5 summarizes the control types and the classification of the MIT presented in this section.

\begin{tabular}{|c|c|c|}
\hline \multicolumn{3}{|l|}{ Classifications of insulators } \\
\hline Types & \multicolumn{2}{|c|}{ Origin } \\
\hline Band insulator & \multicolumn{2}{|c|}{ Under the framework of conventional band theory } \\
\hline Mott-Hubbard insulator & \multicolumn{2}{|c|}{ MIT occurs due to electron-electron interactions } \\
\hline Peierls insulator & \multicolumn{2}{|c|}{ MIT occurs due to electron-phonon interactions } \\
\hline Anderson insulator & \multicolumn{2}{|c|}{ MIT occurs due to disorder-induced localization } \\
\hline \multicolumn{3}{|l|}{ Control mechanisms of MIT } \\
\hline Control type & Representative approach & Materials \\
\hline Bandwidth control & Re cation substitution & $\mathrm{ReNiO}_{3}(\mathrm{Re}=\mathrm{La}, \mathrm{Nd})$ \\
\hline Band-filling control & Chemical doping $(\mathrm{Sr}, \mathrm{O})$ & $\mathrm{YBa}_{2} \mathrm{Cu}_{3} \mathrm{O}_{7-\delta}, \mathrm{La}_{1-\mathrm{x}} \mathrm{Sr}_{x} \mathrm{MnO}_{3}$ \\
\hline
\end{tabular}

FIGURE 1.5: Classification of insulators and control mechanisms of metal-insulator transition. Adapted from [56].

\subsubsection{Metal-Insulator Transition for Resistive Switching applications}

By proper engineering the growth of the material, one can design and tune its electronic properties in such a way that the MIT lies close to the operating temperature [78]. Ideally, a material designed in that way could easily undergo a resistance switch, since the proximity to the MIT can induce a colossal resistance change with a small variation of the carrier density upon the application of an electric field, which should lead to low operating voltages. In the last years the study of RS in Mott systems has gained some attention due to its unique opportunities and huge potential for applications [47, 56, 79-81]. An extensive review on this topic and its potential has been recently published by Janod et al [32]. In this thesis, we propose the use of perovskite Mott systems that are in the metallic state at room temperature to reversibly drive them into the insulating state, upon the application of an electric field, as shown in figure 1.6. We demonstrate that a volumetric resistive transition of the whole material is achieved. In the following section, a concise description of the materials studied in this thesis will be presented. 


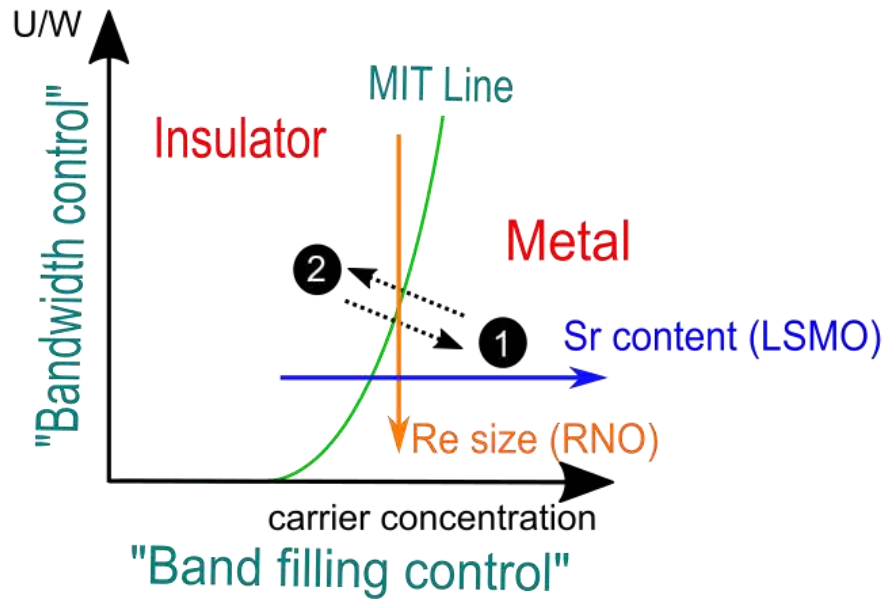

FIGURE 1.6: The diagram shows the relation between U/W ("bandwidth control") and carrier concentration ("bandfilling control"). The green line represents the MIT line. Position 1 and 2 represent the initial and final state respectively. Black dotted lines represent the switch from 1 to 2 under the application of an electric field, and therefore, the RS is induced by the MIT.

\subsection{Metallic perovskite oxides displaying MIT}

Perovskite compounds, which belong to the broader category of strongly correlated complex oxides, are a fascinating class of materials in which the interactions between the different degrees of freedom, such as charge, spin, orbital and lattice effects, strongly compete with each other and lead to exotic physical properties. Strongly correlated oxides can show ferroelectricity [11], multiferroicity [82], ferromagnetism [83], metal-insulator transitions [55], colossal magnetoresistance [84] and high temperature superconductivity [85], paving the way to a wide range of new functionalities that might enhance the performance of actual devices and bring new opportunities.

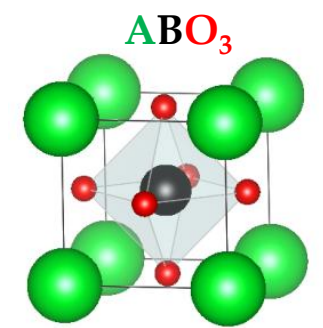

FIGURE 1.7: $\mathrm{ABO}_{3}$ cubic perovskite unit cell.

A perovskite compound has the chemical formula $\mathrm{ABO}_{3}$, where $\mathrm{A}$ and $\mathrm{B}$ are cations, $\mathrm{B}$ being generally a $3 \mathrm{~d}$ transition metal. At high temperatures, the structure is usually cubic (see figure 1.7). However, deviations from an ideal cubic structure might occur, thus reducing the symmetry of the system. The tendency to deviate from the ideal cubic structure can be quantified with the so called Goldsmith tolerance factor (TF), which is defined as

$$
\mathrm{TF}=\frac{\mathrm{R}_{\mathrm{A}}+\mathrm{R}_{\mathrm{O}}}{\sqrt{2}\left(\mathrm{R}_{\mathrm{B}}+\mathrm{R}_{\mathrm{O}}\right)}
$$


Where $R_{A}, R_{B}$ and $R_{O}$ are the radius of the $\mathrm{A}$ cation, the $\mathrm{B}$ cation and the oxygen anion, respectively. The TF is a relative measure of the ionic bond distances $\mathrm{r}_{A-O}$ and $\mathrm{r}_{B-O}$. The value of $\mathrm{TF}=1$ corresponds to a ideally perovskite structure with no distortions. In general, TF deviates from 1 and therefore the structure tends to accommodate with lower symmetry. Typical values to stabilize the perovskite structure range between $0.89<\mathrm{TF}<1.02$. If $\mathrm{TF}>1$, the system has a tendency towards a polar distortion, where a displacement of the cation with respect to the oxygen anion produces an electrical dipole, which might induce ferroelectricity. In the case where TF $<1$, non-polar instabilities are more common and the oxygen octahedra surrounding the $\mathrm{B}$ cation might either rotate or buckle, thus reducing the so-called superexchange angle B-O-B. This angle is particularly relevant in materials where conduction or magnetism originates from states that overlap between the $3 \mathrm{~d}$ orbitals of the $\mathrm{B}$ cation and the $2 \mathrm{p}$ orbitals of the $\mathrm{O}$ anion. This is the case for the three perovskite families studied in this thesis: manganites, rare-earth nickelates and the superconducting cuprate $\mathrm{YBa}_{2} \mathrm{Cu}_{3} \mathrm{O}_{7-\delta}$.

\subsubsection{Lanthanum strontium manganites}

The term manganites makes reference to a family of perovskite compounds under the general formula of $\mathrm{A}_{1-x} \mathrm{~B}_{x} \mathrm{MnO}_{3}$ where $\mathrm{A}$ and $\mathrm{B}$ can be a trivalent or divalent element, mostly rare earth (e.g., $\mathrm{La}, \mathrm{Nd}, \mathrm{Pr}, \mathrm{Sm}$ ) or alkaline earth (e.g, $\mathrm{Sr}, \mathrm{Ca}, \mathrm{Ba}, \mathrm{Pb})$, respectively. For this reason, they are also known as mixedvalence manganites. Although manganites were discovered in the 1950's [86], the discovery of the "colossal" magnetoresistance effect $[87,88]$ triggered enormous interest in the last decades [84, 89-91]. Depending on the composition of the cations A and B the material can show a variety of physical properties, such as ferromagnetism, antiferromagnetism, charge and orbital ordering or metalinsulator transition [90]. Among the whole family, lanthanum strontium manganites, $\mathrm{La}_{1-x} \mathrm{Sr}_{x} \mathrm{MnO}_{3}$ (LSMO), present metal-insulator transition tunable with the doping level linked to a ferromagnetic-paramagnetic transition. This composition is widely studied for its metallic-ferromagnetic behaviour at room temperature. In the following, the most relevant properties of LSMO for this thesis are presented.

\section{Cystral and electronic structure of LSMO}

Lanthanum strontium manganites crystallize in a cubic structure with the Mn atom occupying the centre site of an oxygen octahedron while La and Sr cations occupy the corners of the unit cell, as shown in figure 1.8.

Since the A site of the $\mathrm{ABO}_{3}$ perovskite structure is partially occupied by two different atoms, $\mathrm{La}^{3+}$ and $\mathrm{Sr}^{2+}$, the $\mathrm{Mn}$ cation presents a mixed-valence state, $\mathrm{Mn}^{3+}$ and $\mathrm{Mn}^{4+}$. Thus, the TF is strongly affected due to the different ionic radii of the species, modifying the Mn-O-Mn bond angle, which is a key parameter to explain the physical properties of LSMO. The crystal field generated by the oxygen octahedron partially raises the degeneracy of the $3 \mathrm{~d}$ Mn multiplet, splitting it into a low energy 3 -fold degenerate state $t_{2 g}$ and a high energy 2 -fold degenerate state $e_{g}$. Figure 1.9 shows a basic scheme of the $3 \mathrm{~d}$ atomic levels of the $\mathrm{Mn}^{3+}$ ion. In addition, there is an extra distortion arising from electronic interactions in these systems known as the Jahn-Teller (J-T) distortion [92]. An spontaneous elongation or compression of the oxygen octahedron along the axial direction occurs. This means 


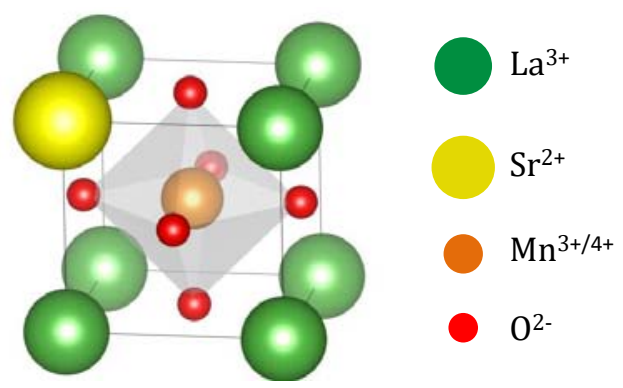

FIGURE 1.8: Basic scheme of LSMO crystal structure. Sr or La occupy the corners of the unit cell. Mn atom is located at the centre of the unit cell coordinated with an oyxgen octahedron.

an extra lift of the degeneracy of the $t_{2} g$ and $e_{g}$ levels in the $\mathrm{Mn}^{3+}$ ions. In contrast, this degeneracy does not occur in the $\mathrm{Mn}^{4+}$ where the $e_{g}$ levels are unoccupied.
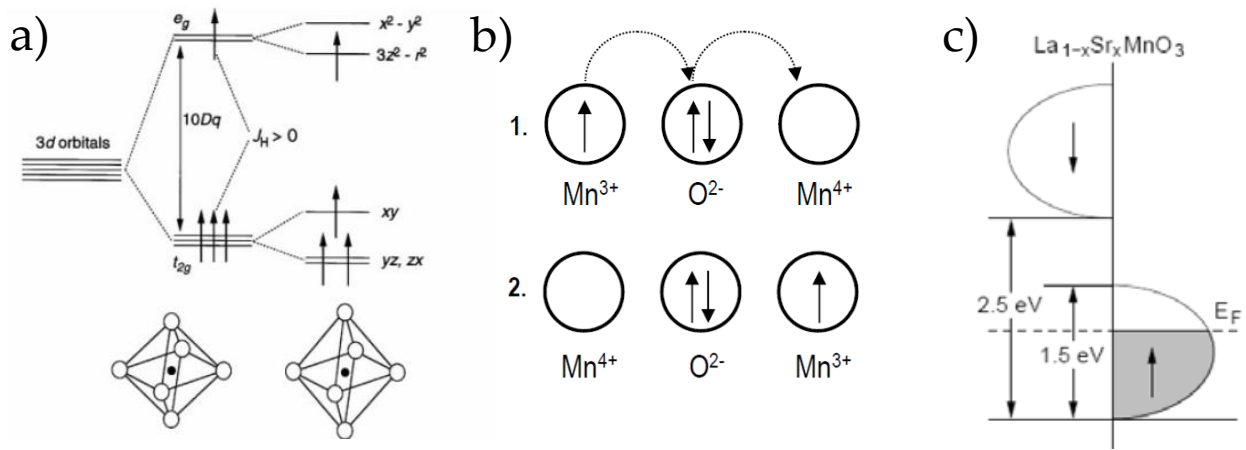

FIGURE 1.9: a) 3d atomic levels of the $\mathrm{Mn}^{3+}$ ion with the different degeneracy levels. Adapted from [91] b) Schematic representation of the Double Exchange mechanism. Taken from [84] c) Band diagram of LSMO. Taken from [89]

The lower energy $t_{2 g}$ electrons are less hybridized with oxygen $2 p$ orbitals and therefore strongly localized. Contrariwise $e_{g}$ electrons can be itinerant and consequently, responsible for the electric conduction, providing the presence of empty $e_{g}$ states in the system. To generate $e_{g}$ empty states hole-doping is required, i.e., to introduce $\mathrm{Mn}^{4+}$ into the crystal by substituting the trivalent La for a divalent alkaline earth (in our case, $\mathrm{Sr}$ ).

These electronic features are key elements to describe the link between ferromagnetism and metallicity in these compounds, both constricted by the large exchange energy $\left(J_{H} \sim 2-3 \mathrm{eV}\right)$ [84] or the large on-site Hund coupling between Mn $3 d$ electrons. The Double Exchange (DE) mechanism [93] consists of two simultaneous electron hopping processes. In the first, one electron in the $\mathrm{Mn}^{3+}$ ion jumps from an $e_{g}$ state to a neighbouring oxygen $2 p$ orbital. In the second, a second electron from this same oxygen $2 p$ orbital and with parallel spin is transferred to a contiguous $\mathrm{Mn}^{4+}$ ion with an unoccupied $e_{g}$ state, as depicted in figure $1.9 \mathrm{~b}$. The probablity of the $e_{g}$ electron transfer from a $\mathrm{Mn}^{3+}$ to a $\mathrm{Mn}^{4+}$ is basically proportional to $\cos \left(\frac{\theta}{2}\right)$, being $\theta$ the angle between the Mn spins. Thus, the electron hopping is larger when spins are parallel, i.e., in a ferromagnetic configuration, and zero if they are in an anti-parallel configuration.

In particular, $\mathrm{La}_{1-\mathrm{x}} \mathrm{Sr}_{\mathrm{x}} \mathrm{MnO}_{3}$ has a strong double exchange mechanism and therefore, the $e_{g}$ electrons become delocalized in the stabilized ferromagnetic phase 
for a certain range of doping, centered around $x=0.3$. An schematic diagram of the electronic band structure [91] in LSMO is shown in figure 1.9 (c). The spin up and down bands are separated by a gap of $1 \mathrm{eV}$. The upper band (spin down in the scheme) is empty, leading to a half-metallic ferromagnetic behaviour. $\mathrm{La}_{1-\mathrm{x}} \mathrm{Sr}_{\mathrm{x}} \mathrm{MnO}_{3}$ with doping $x<0.5$ has a conduction band more than half-filled (and therefore, carriers are holes) while if $x>0.5$ the conduction band is less than half-filled and the carriers are electrons.

\section{Phase diagram of $\mathrm{La}_{1-x} \mathrm{Sr}_{x} \mathrm{MnO}_{3}$}

Despite its simplicity and usefulness, the briefly introduced DE mechanism cannot account for the rich phase diagram and variety of phenomena observed in the manganites systems. Further considerations should be made to fully understand the underlying physics, such as collective Jahn-Teller distortions, charge/orbital ordering or antiferromagnetic superexchange. In fact, the complexity of the manganites phase diagram emerge from all these interactions and their mutual competition.

Strongly correlated systems such as manganites can be characterized by two main parameters: the electron hopping amplitude $t$ and the band-filling $n$ (the density of carriers). Both parameters can be varied by changing the chemical composition of the compound. For example, modifying the average cation radius leads to a change in the Mn-O-Mn bond angle which directly affects the hopping amplitude $t$ [94]. On the other hand, by varying the doping level $x$, changes in the band filling $\mathrm{n}(\mathrm{n}=1-\mathrm{x})$ can be achieved.

a)

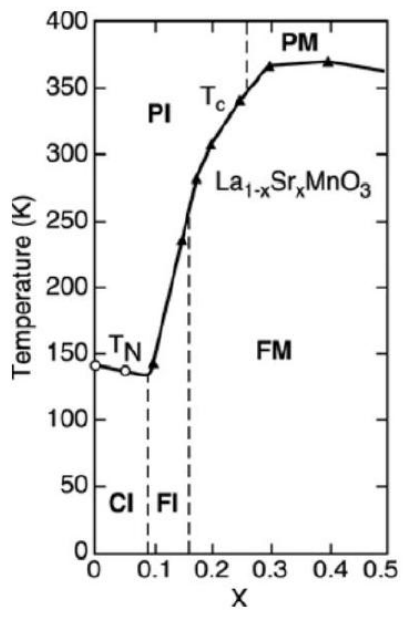

b)

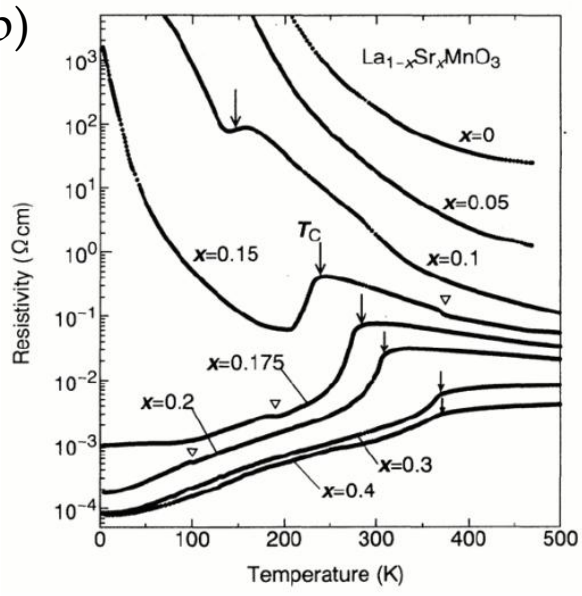

FIGURE 1.10: (a) Magnetic phase diagram of $\mathrm{La}_{1-x} \mathrm{Sr}_{x} \mathrm{MnO}_{3}$ showing the transition temperature as a function of the doping $\mathrm{x}$. Adapted from [91]. (b) Resistivity as a function of temperature in zero field $\mathrm{La}_{1-\mathrm{x}} \mathrm{Sr}_{\mathrm{x}} \mathrm{MnO}_{3}$ for various $\mathrm{x}$ content. Arrows indicate the $T_{c}$ and open triangles represent structural transitions. Taken from [73]

Figure 1.10 (a) shows the phase diagram of $\mathrm{La}_{1-x} \mathrm{Sr}_{x} \mathrm{MnO}_{3}$ [91]. It shows a stable ferromagnetic state for $0.16<x<0.5$, with a maximum in the temperature of the ferromagnetic-paramagnetic transition, or Curie temperature, $\mathrm{T}_{c}$, at $x=0.3$, where $T_{c} \sim 360 \mathrm{~K}$. Other phases are observed in the diagram, such as paramagnetic metallic (PM), paramagnetic insulating (PI) and spin-canted insulating (CI). 
The doping level in $\mathrm{La}_{1-x} \mathrm{Sr}_{x} \mathrm{MnO}_{3}$ strongly influences the temperature dependence of the resistivity [73]. At low doping levels, LSMO remains insulating $(d \rho / d T<0)$ for the full temperature range. As the doping level is increased by introducing $\mathrm{Mn}^{4+}$ into the system, a metal-insulator transition occurs. This transition occurs at different temperatures $\left(\mathrm{T}_{\mathrm{M}-\mathrm{I}}\right)$ as a function of the doping level, as stated in figure 1.10 (b). As a first approach, MIT in manganites can be understood with the Current Carrier Density Collapse (CCDC) model $[95,96]$. The CCDC model states that the MIT is driven by an exchange interaction of polaronic carriers with localized spins. It also accounts for a huge decrease in the number of charge carriers during the transition, which is a consequence of bound pairs formation in the paramagnetic phase.

\subsubsection{Rare-earth nickelates}

The rare-earth nickelates are mainly known for their sharp temperature-driven metal-insulator transition with a resistance change of orders of magnitude. As it was shown in a previous section, the MIT of these systems is bandwidth-controlled. The stabilization of the $\mathrm{Ni}^{3+}$ state in these systems requires very high temperatures and high pressures, and therefore, these materials are difficult to synthesize in its bulk form. Initially, research studies were conducted in powdered polycrystalline samples. Single crystals of nickelates with rare earths ranging from La to Sm have so far only been achieved via thin film growth by either physical [71, 97-100] or chemical [101, 102] methods. Nickelates are therefore a relatively young research field and it evolves rapidly. In the following, a concise summary of their main properties will be presented. More detailed reviews on these fascinating materials can be found in references [68, 103].

\section{Crystal and electronic structure of $\mathrm{RENiO}_{3}$}

The rare earth nickelates (RENO) present a perovskite structure with the generic formula $\mathrm{RENiO}_{3}$ (see figure 1.11), where $\mathrm{RE}$ is a rare earth and $\mathrm{Ni}$ is in its $3+$ low spin state with fully occupied $\mathrm{t}_{2 g}$ bands and one electron in the $\mathrm{e}_{g}$ band. Conduction is believed to occur due to the hybridization of the Ni $3 \mathrm{~d}$ and $\mathrm{O} 2 \mathrm{p}$ orbitals.

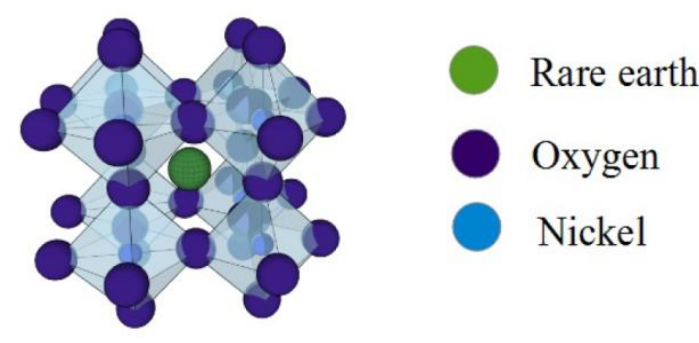

FIGURE 1.11: Perovskite structure, for rare-earth nickelates, where the rare earth cation occupies the central position and $\mathrm{Ni}$ cations are surrounded by the oxygen octahedron.

Apart from $\mathrm{LaNiO}_{3}(\mathrm{LNO})$, which behaves as a paramagnetic metal at all temperatures with a rhombohedral $R \overline{3} c$ structure in the bulk, perovskite nickelates exhibit a MIT from a high temperature orthorhombic $(\mathrm{Pbnm})$ metallic phase to a low temperature monoclinic $\left(P 2_{1} / n\right)$ insulating phase. It is worth to mention that MIT has 
been also reported in ultrathin LNO films [100]. Two phenomena characterize the ground state of these systems. The first is related to a charge ordering, also called charge disproportion. The $\mathrm{Ni}$ sites splits their unique $3+$ valence in the metallic phase, into two adjacent $\mathrm{Ni}$ sites in the insulating phase with respective valences of $3+\delta$ and $3-\delta$. Charge disproportion can be expressed as $2 \mathrm{Ni}^{3+} \rightarrow \mathrm{Ni}^{3+\delta}+\mathrm{Ni}^{3-\delta}$ where $\delta$ is below 1 and depends on the distortion (rare earth cation) and the temperature. This phenomenon has been observed in the whole nickelates family in the bulk $[104,105]$ and for $\mathrm{NdNiO}_{3}$ thin films [106]. The second phenomenon is a peculiar antiferromagnetic ordering that develops in all nickelates [104,107]. In the pseudocubic notation, the spins in the (111) planes are ferromagnetically coupled, while they are stacked in a $\uparrow \uparrow \downarrow \downarrow$ fashion along the [111] direction.

\section{Phase diagram of $\mathrm{RENiO}_{3}$}

The physical properties of the nickelates present a strong dependency on rare earth cation, which makes out of these materials a canonical example of a bandwidthcontrolled MIT [68, 69], as shown on figure 1.12 (a). By choosing a smaller rare earth, the tolerance factor defined in equation 1.1 and subsequently the Ni-O-Ni bonding angle are decreased and the transition temperature $\mathrm{T}_{M I}$ (or $\mathrm{T}_{\mathrm{MIT}}$ ) between the metallic and the insulating phase increases almost linearly, as observed in figure 1.12 (b). As previously noticed, $\mathrm{LaNiO}_{3}$ does not present MIT, since it has the largest tolerance factor, with a rhombohedral structure instead of an orthorhombic. The $180^{\circ} \mathrm{Ni}-\mathrm{O}-\mathrm{Ni}$ bonding angle produces a large orbital overlapping preventing the gap opening, except for ultrathin films [100].
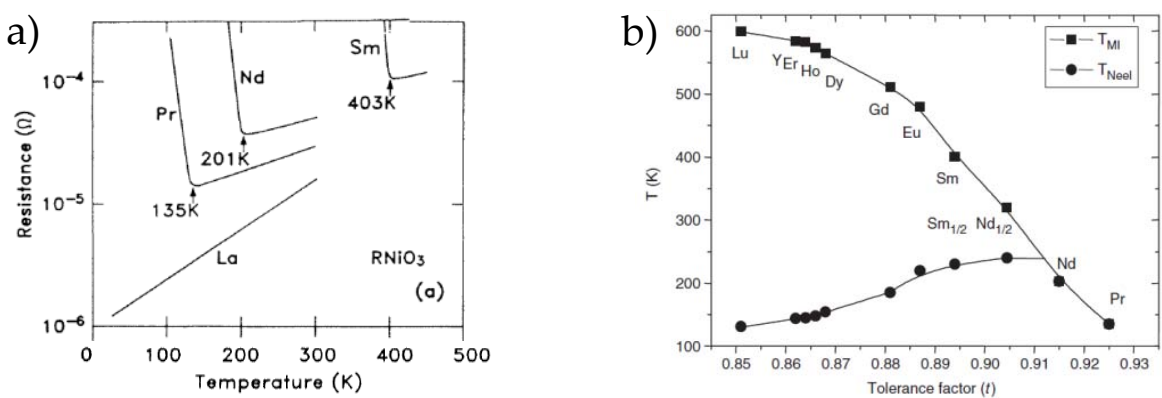

FIGURE 1.12: (a) Resistivity curves and (b) magnetic transitions temperature as a function of the tolerance factor $t$. Taken from [103].

Reducing the Ni-O-Ni angle leads to a decrease of the bandwidth, and therefore, to an increase of the U/W strength, which drives the system deeper into the insulating state. Thus, even though the rare earth cation is not directly connected to the electronic properties, it enables to control the bandwidth by 30-40\% [55]. In the antiferromagnetic phase, two regimes are observed. In the first part of the diagram, the Néel temperature $\mathrm{T}_{N}$ of the antiferromagnetic ordering coincides with the $\mathrm{T}_{\mathrm{MIT}}$ and also increases with increasing $\mathrm{U} / \mathrm{B}$ strength. This is the case for $\mathrm{PrNiO}_{3}$ and $\mathrm{NdNiO}_{3}$. For Sm and smaller rare earth, $\mathrm{T}_{N}$ is decoupled from $\mathrm{T}_{\mathrm{MIT}}$ and decreases with increasing $\mathrm{U} / \mathrm{W}$ strength or equivalently, decreasing tolerance factor. Therefore, two transitions can be observed: from a paramagnetic metallic to a paramagnetic insulating phase at high temperatures, and eventually to an antiferromagnetic insulating phase at lower temperatures [108]. 
The phase diagram gets even more complex for thin films. In particular, by selecting the film/substrate lattice misfit, substrate symmetry and orientation and dimensionality of the film, the $\mathrm{Ni}-\mathrm{O}$ bond lengths and the $\mathrm{Ni}-\mathrm{O}-\mathrm{Ni}$ bond angles can be tuned. These distortions can modify the crystal field and bandwidth of the rare-earth nickelate compounds [71, 78, 100].

\subsubsection{Cuprate $\mathrm{YBa}_{2} \mathrm{Cu}_{3} \mathrm{O}_{7-\delta}$}

High temperature superconductors (HTS) have attracted worldwide attention since the discovery by Bednorz and Muller in 1986 [85] of superconducting copper oxide perovskite structures. Despite intensive research in these 30 years since HTS were discovered, a general theory explaining superconductivity in these materials remains elusive. Because these compounds have transition temperatures much higher than conventional superconductors, they have opened the possibility to use them in technological applications at temperatures above the boiling point of liquid nitrogen and thus, reducing the cost of the cryogenics involved by working at temperatures in the range of 65-77 K. Many exciting applications have been proposed, such as quantum computing [109], medical imaging [110], superconducting electronics [111] and radioastronomy [112]. However, HTS are mostly known for their excellent capabilities of conducting high currents at high magnetic fields. In particular, among the series of superconducting cuprates, $\mathrm{YBa}_{2} \mathrm{Cu}_{3} \mathrm{O}_{7-\delta}(\mathrm{YBCO})$ presents the best performance and huge efforts are being done to trigger $\mathrm{YBCO}$ coated conductors (CC's) for power applications [113, 114].

\section{Crystal and electronic structure of $\mathrm{YBa}_{2} \mathrm{Cu}_{3} \mathrm{O}_{7-\delta}$}

YBCO has a triple perovskite structure composed of $\mathrm{BaCuO}_{3}$ and $\mathrm{YCuO}_{2}$ units. The structure is formed by a succession of $\mathrm{CuO}_{2}$ planes lying normal to the c-axis. These planes play a key roles, since the transport of charge carriers occurs in the planes and superconductivity (SC) is believed to take place there. $\mathrm{CuO}_{2}$ planes are separated by charge-reservoir interleaved layers which contain $\mathrm{CuO}_{x}$ chains. In the chains the oxygen content is not fixed and it determines the crystallographic structure that $\mathrm{YBCO}$ adopts. The oxygen concentration of the chains defines the stoichiometry of the compound, and it is denoted with $x=7-\delta$ in the $\mathrm{YBa}_{2} \mathrm{Cu}_{3} \mathrm{O}_{7-\delta}$ unit cell formula. The charge transfer occurring between the chains and the planes results in a very complex phase diagram [115], as shown in figure 1.13.

a)

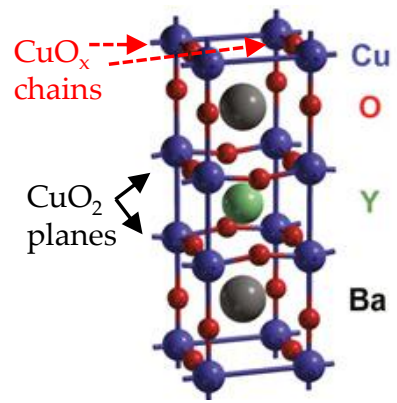

b)

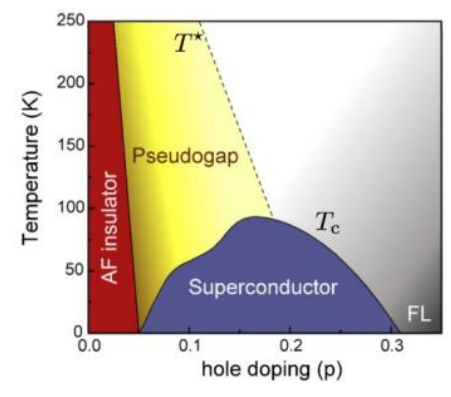

FIGURE 1.13: Crystal structure of YBCO. Taken from [116].

When there is no oxygen in the chains $(\delta=0), \mathrm{YBCO}$ is an antiferromagnetic Mott insulator adopting a tetragonal $P 4 / \mathrm{mmm}$ structure. By progressively changing the 
oxygen content in the chains, the $\mathrm{CuO}_{2}$ planes are doped with holes providing free carriers to the Mott insulator, and eventually the compound becomes a strange metal with superconducting behaviour and a structural transition to an orthorhombic Pmmm structure occurs. This MIT is achieved via band-filling control.

The intrinsic anisotropy of the cuprates due to their layered structure is directly observed in the transport properties. The conductivity is confined in the a-b planes. Along the c-axis normal conductivity is typically three orders of magnitude smaller than in the a-b planes. Among the cuprates, YBCO has the lowest anisotropy and therefore, very appealing from the point of view of applications.

\section{Superconducting properties of $\mathrm{YBa}_{2} \mathrm{Cu}_{3} \mathrm{O}_{7-\delta}$}

In the following, the main structural and superconducting properties of YBCO will be presented. For a deeper understanding of the basics of superconductivity, the reader is referred to the following references $[117,118]$.

YBCO is a high-temperature type II superconductor. Superconducting materials are characterized by their ability to carry electrical current without energy dissipation when cooling them below a certain temperature known as critical temperature $\mathrm{T}_{c}$ (In YBCO, $T_{c} \approx 93 K$ ). This transition to a zero-resistance state is schematically depicted in figure 1.14 (a). Another specific characteristic of type II superconductors is their complex magnetic phase diagram [119]. The figure 1.14 (b) schematically shows the magnetic phase diagram in YBCO. In the first region, below a first critical field known as lower critical field, $\mathrm{H}_{c 1}$, the applied magnetic field is expelled from the interior of the superconductor. This is called the Meissner effect.

a)

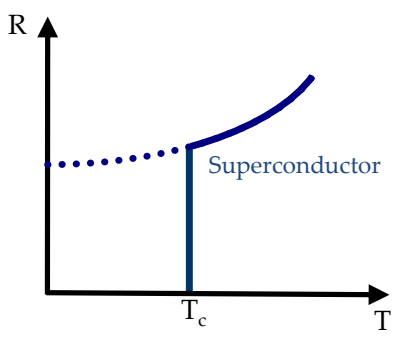

b)

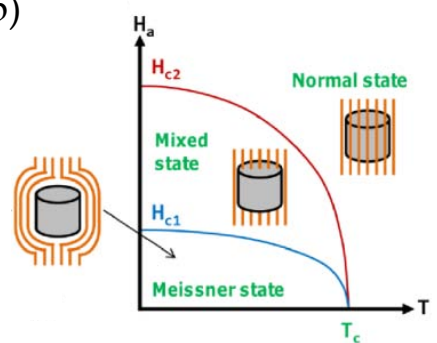

FIGURE 1.14: a) Scheme of a superconducting transition at $\mathrm{T}_{c}$. b) Magnetic phase diagram in type-II superconductors.

Then, at much higher fields, a second critical field known as upper critical field $\mathrm{H}_{c 2}$ appears. Above, the superconducting state is lost and the transition to the normal state occurs. In the region between $\mathrm{H}_{c 1}$ and $\mathrm{H}_{c 2}$, the magnetic flux penetrates into the superconductor forming quantized cylindrical flux lines called vortices. This region is known as the mixed state. Vortices are non-superconducting nanometersize entities, and therefore, when a current is applied to the supeconductor, vortices will feel a Lorentz like force, $F_{L}$, according to:

$$
\overrightarrow{F_{L}}=\vec{J} x \vec{B}
$$

where $\vec{J}$ is the current density and $\vec{B}$ is the magnetic field into the superconductor. This force will induce vortex motion and thus producing dissipation of energy. 
However, there are different kinds of nanometric intrinsic defects in the superconducting matrix that are able to pin the vortices and immobilize them, such as low angle grain boundaries, stacking faults, dislocations, twin boundaries [114, 120]. In addition, artificial pinning centres can be added to the crystal structure in order to enhance vortex pinning and consequently, the critical current density, $\mathrm{J}_{c}$ of the material. This critical current density, $\mathrm{J}_{c}$, is defined as the maximum current that the superconductor can carry without energy dissipation due to vortex motion.

As it was explained above, superconductivity emerges in the phase diagram upon hole doping in the $\mathrm{CuO}_{2}$ planes. The doping level depends on the oxygen content of the $\mathrm{CuO}_{x}$ chains and strongly determines the superconducting properties. Figure 1.15 shows the $\mathrm{T}_{c}$ dependence on the oxygen content $\delta$. Therefore, a nanoscale control of the oxygen content could enable the generation of micro- or nanoregions where the superconducting state has been diminished (or even suppressed) through the metal-insulator transition of YBCO. These non-superconducting regions might act as suitable pinning sites for vortices under applied magnetic field.

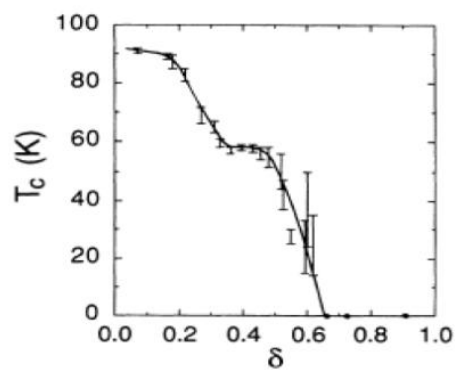

FIGURE 1.15: Critical temperature of YBCO crystals as a function of the oxygen content. Taken from [121]

Resistive switching effect has been previously reported in YBCO. A deeper review on this field is presented in chapter 4. Therefore, in this thesis the study of the resistive switching properties is twofold justified. Its RS behaviour at RT is interesting enough to justify its investigation for RRAM applications. Besides, the interaction of RS states and the superconducting matrix below $\mathrm{T}_{c}$ might pave the way for future reconfigurable pinning sites for fluxonic devices.

\subsubsection{Cerium dioxide}

Cerium dioxide $\left(\mathrm{CeO}_{2}\right)$ is briefly overviewed in this section in order to settle its excellent properties as ion conductor. In this thesis, $\mathrm{CeO}_{2}$ is used as a thin capping layer to modify the resistive switching properties of the $\mathrm{La}_{1-\mathrm{x}} \mathrm{Sr}_{x} \mathrm{MnO}_{3}$ and $\mathrm{YBa}_{2} \mathrm{Cu}_{3} \mathrm{O}_{7-\delta}$ films, thus creating bilayer systems. $\mathrm{CeO}_{2}$ adopts a fluorite structure [122] (see figure 1.16) where the $\mathrm{Ce}^{4+}$ cations occupy face-centered positions in a cubic unit cell and the $\mathrm{O}^{2-}$ anions occupy the eight tetrahedral sites in between.

The functional properties of $\mathrm{CeO}_{2}$ heterostructures make it a very appealing material for scientific and technological applications. For instance, it can be used in electronics due to its compatibility with silicon, high chemical and mechanical stability and high dielectric constant of $\kappa \sim 26$ [79, 123, 124]. In addition, it has been chosen as a buffer layer for HTS coated conductor applications [125]. Finally, $\mathrm{CeO}_{2}$ also 


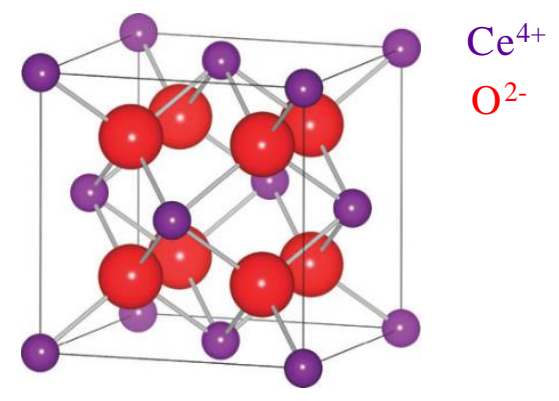

FIGURE 1.16: The fluorite crystal structure adopted by $\mathrm{CeO} 2$. Large red spheres represent $\mathrm{O}^{2-}$ ions and small purple spheres $\mathrm{Ce}^{4+}$ ions. Taken from [122].

presents remarkable catalytic properties and high oxygen diffusion. These characteristics can be exploited in fuel cells and sensing devices [122, 126, 127]. Under reducing conditions, non-stoichiometric $\mathrm{CeO}_{2-y}$ can be formed by oxygen release and consequently, $\mathrm{Ce}^{4+}$ is reduced to $\mathrm{Ce}^{3+}$, with the concomitant formation of oxygen vacancies within the crystal structure, and thus increasing its conductivity. [128, 129]. This reaction can be expressed as

$$
\mathrm{CeO}_{2} \rightarrow \mathrm{CeO}_{2-y}+\frac{y}{2} \mathrm{O}_{2}
$$

This process leads to modifications of $\mathrm{CeO}_{2}$ to $\mathrm{CeO}_{2-x}$ out of stoichiometry and will be used along this thesis to act as an oxygen exchange layer, i.e. as an oxygen reservoir in the proposed bilayer devices upon the application of an electric field to the bilayer.

\subsection{Outline of the thesis}

The resistive switching phenomena observed in a large variety of oxides might play a key role in the future non-volatile memories. Its outstanding performance (writing, reading and erasing speeds below the $<100$ ns regime) and its potential scalability make out of this effect a very appealing candidate for an universal memory which overcomes the limitations of actual technologies. Besides this, the RS effect might find more niches of applications such as memristor-based reconfigurable electronics and neuromorphic computing architectures.

Despite its potentiality, the lack of understanding of the underlying physical mechanism is one of the main issues to overcome for widespread application of this technology. The origin of the RS is determined by the oxide material employed. In this regard, we propose a novel approach based on the use of metallic perovskite oxides with strong electron-electron interaction: $\mathrm{La}_{1-x} \mathrm{Sr}_{x} \mathrm{MnO}_{3}, \mathrm{ReNiO}_{3}$ and $\mathrm{YBa}_{2} \mathrm{Cu}_{3} \mathrm{O}_{7-\delta}$. These systems present a metal-insulator transition, an intrinsic feature which might be the ultimate responsible of the RS effect in these compounds and might boost the performance of the devices and show extra functionalities, as superconductivity, ferromagnetism and colossal magnetoresistance. In addition, the metallic character of the films might facilitate its device integration due to its capability of being the active element and the word line connectors in the integrated circuit. 
The manuscript has been divided into five chapters. The present Chapter 1 presents a brief introduction to the resistive switching phenomena, including the device phenomenology according to the polarity operation and the physical mechanism behind the effect. We also present a general overview of the metal-insulator transition mechanisms and a short introduction regarding the materials employed, with special emphasis in the control of their MIT. The role of $\mathrm{CeO}_{2}$ as oxygen reservoir is also exposed.

In chapter 2, we present the growth methodologies and the processing of the four compounds involved in this thesis. Their structural, morphological and physical properties are also presented. The main tools employed to induce and/or characterize the resistive switching behaviour of the films are discussed: Atomic Force Microscopy, Scanning Tunnelling Microscopy and macroscopic electrical characterization in probe stations. The measurement procedure and precautions adopted to ensure reproducible and reliable RS behaviour are discussed.

In chapter 3, the resistive switching properties of $\mathrm{La}_{1-x} \mathrm{Sr}_{x} \mathrm{MnO}_{3}$ thin films studied by C-AFM are presented. I-V point contacts and micrometric arrays are used to propose a volume switching mechanism based on the MIT through oxygen exchange with the atmosphere. Evidences supporting this proposal are presented by tuning the RS parameters through material processing with the Sr content and transport properties of RS areas. Atmosphere dependent measurements are performed in LSMO films and $\mathrm{CeO}_{2} / \mathrm{LSMO}$ bilayer systems. Finally, a three-terminal configuration is used to demonstrate the potential of these materials in future nanoelectronics.

Chapter 4 can be divided into two different subchapters. The first subchapter comprises our investigations on YBCO thin films. It includes the basic RS phenomenology and its relation with microstructural features. In addition, our efforts towards reconfigurable vortex pinning sites are presented. Finally, the second subchapter contains our investigation on $\mathrm{RENiO}_{3}$. A fine correlation between structural defects of our CSD-RENO films and the observed resistive switching behaviour is presented. As a comparison, we have also investigated the point-contact RS properties of three $\mathrm{RENiO}_{3}$ films grown by a RF-Sputtering, which present to a different defect landscape.

The main conclusions of our research are summarized in Chapter 5. The characterization techniques employed during this thesis has been including as an appendix at the end on the manuscript. 



\section{Chapter 2}

\section{Experimental procedure}

In this chapter, we detail the experimental methodology used for the preparation of the different metallic perovskite oxides and bilayer systems employed. It contains a description of the growth methodologies used in this thesis, the structural, morphological and physical properties characterization of the thin films and the selection and surface conditioning of substrates. Lithography and metal electrode evaporation processes are also presented. Due to the key role that scanning probe microscopy techniques have played in this thesis, a detailed description of their work principle and setup configuration is also provided in this chapter. Finally, a list of the characterization techniques described in the Appendix A and my implication in each one is also mentioned.

\subsection{Sample preparation}

In this section, we report the procedure followed to prepare the samples for resistive switching measurements. It includes the growth and microstructural characterization of the metallic perovskite thin films employed in this thesis and eventual lithography and metal deposition processes. A brief introduction to the growth techniques and my contribution to each step is also commented.

\subsubsection{Substrate selection and preparation}

In the past, substrates were considered a passive element supplying only mechanical support. However, they have been shown to act as an active template during the epitaxial growth of materials. In particular, interfacial effects at the nanoscale have been demonstrated to play a key role in the development of new and exotic functionalities[130,131], and consequently, great efforts have been done to control the quality and the sharpness of both surface and interfaces [132, 133].

Nucleation and growth of oxide heterostructures are determined by the presence of the substrate below, and therefore, their crystalline structure and surface characteristics are essential to determine the final properties and morphology of the system. During the development of this thesis, two different single-crystalline oxide substrates with perovskite structure have been used in the growth of the epitaxial thin films, namely $\mathrm{SrTiO}_{3}$ (STO) and $\mathrm{LaAlO}_{3}$ (LAO).

$\mathrm{SrTiO}_{3}$ Strontium titanate (STO) is a paradigmatic example of cubic perovskite structure $\left(\mathrm{ABO}_{3}\right)$, with $\mathrm{A}=\mathrm{Sr}$ and $\mathrm{B}=\mathrm{Ti}$ ) at room temperature (space group 
$P m \overline{3} m$. STO single crystal substrates have been widely used for its compatibility due to the low lattice mismatch with the active layers in different applications, such as high temperature superconductors [134, 135], ferroelectricity [136] and ionic conductivity [131]. Furthermore, STO itself present very interesting properties as a functional material. For instance, it can be employed as gate dielectric material due to its high- $k$ and can be integrated on silicon without outgrowth of silicon dioxide [137], and even promoting the integration of other perovskite oxides on silicon [138]. In the resistive switching community, STO has been widely studied [25, 26, 139, 140]. According to our supplier (Crystec), STO single crystal substrates have a lattice parameter $a_{S T O}=3.905 \AA$, in good agreement with the values reported in the literature [141] and confirmed by XRD measurements performed in our facilities.

$\mathrm{LaAlO}_{3}$ Lanthanum aluminate (LAO) exhibits an ideal cubic perovskite structure at high temperatures, but goes through a second order phase transition to the rhombohedral $R \overline{3} c$ structure at around $800 \mathrm{~K}$, owing lattice parameters of $a=b=c=5.3547 \AA$ and $\alpha=\beta=\gamma=60.113^{\circ}$. This rhombohedral distortion can be described as a pseudocubic cell with $a_{L A O}=3.79$ Åand $\alpha=90.096^{\circ}$. This phase transition is accompanied by the formation of twin planes to relieve the stress produced by the lattice distortion. An extensive description of twinning in LAO is given by Bueble and co-workers [142]. The supplier (Crystec) provides a lattice parameter of $a_{L A O}=3.82 \AA$, which is close to the literature values $\left.\left(a_{L A O}=3.789\right) \AA\right)$ [142] and to our XRD measurements $\left(a_{L A O}=3.79 \AA\right)$.

In this thesis (001)-oriented LAO and STO substrates of $5 \times 5 \times 0.5 \mathrm{~mm}$ in size and one side polished were employed. The growth of the metallic perovskite oxides studied in this thesis on STO and LAO substrates leads to the growth of epitaxial films due to the the small lattice mismatch between them. Table 2.1 summarizes the strain of the films. This mismatch was calculated using the formula $\epsilon=\frac{a_{\text {substrate }}-a_{\text {film }}}{a_{\text {substrate }}}$ :

\begin{tabular}{|c|c|c|c|}
\hline Compound & $\mathrm{a}(\mathrm{b})$ bulk $(\AA)$ & $\epsilon_{S T O}(\%)$ & $\epsilon_{L A O}(\%)$ \\
\hline LSMO & 3.873 & +0.9 & -2.3 \\
\hline YBCO $^{*}$ & $3.886(3.821)$ & +1.2 & -1.7 \\
\hline LNO & 3.840 & +1.7 & -1.3 \\
\hline NNO & 3.806 & +2.6 & -0.6 \\
\hline
\end{tabular}

TABLE 2.1: Mismatch between substrates and the materials employed in this thesis. *An average cell parameter has been taken to calculate the mismatch to the substrates. 3.853

Surface conditioning to assure a single-terminated and atomically flat morphology is a essential step prior to the deposition and growth of heteroepitaxial functional thin films. Therefore, processing of the as-received substrates is required to achieve a clean, smooth surface, single-terminated and free of impurities surfaces. In our case, the following protocol was applied:

Cleaning procedure The general cleaning procedure for the as-received substrates includes ultrasonication in acetone and methanol during 5 minutes in each solvent. This procedure allows the removal of non-polar and polar adsorbate impurities respectively, while maintaining the stoichiometry and without surface degradation. (This will be the general cleaning procedure applied for all the substrates and samples unless something else is mentioned.) 
Chemical selectivity of substrate termination In the case of STO, we have followed a methodology based on the work of Koster et al. [143]. There, an etching $\mathrm{NH}_{4} \mathrm{~F}-\mathrm{HF}$ solution of controlled $\mathrm{pH}$ is used to obtain single-terminated surface. The process implies the chemical reaction of the Sr-O terminated planes with $\mathrm{CO}_{2}$ and water by cleaning the substrates in Mili-Q purified water. This produces $\mathrm{SrCO}_{3}$ and $\mathrm{Sr}(\mathrm{OH})_{2}$. The latter is dissolved in the $\mathrm{NH}_{4} \mathrm{~F}-\mathrm{HF}$ diluted solution (5:1, from Sigma-Aldrich) for 60-90 s. Afterwards, the acid is removed with a gently bath in Mili-Q water. This process leads to $\mathrm{Ti}-\mathrm{O}_{2}$ single terminated surface.

Surface reconstruction Finally, a thermal treatment is employed to promote the formation of the steps morphology due to the miscut. STO and LAO substrates are placed on an alumina crucible and are introduced in a quartz tube inside a high-temperature tubular furnace. Then, they are heated up to $900^{\circ} \mathrm{C}$ at $15^{\circ} \mathrm{C} \cdot \mathrm{min}^{-1}$ and remain at that temperature for 5 hours under a constant oxygen flow of $0.5 \mathrm{~L} \cdot \mathrm{min}^{-1}$. Cooling to room temperature is performed at a rate of $10^{\circ} \mathrm{C} \cdot \mathrm{min}^{-1}$. In the case of $\mathrm{LAO}$, treatments at higher temperatures are reported in the literature [144] to promote surface reconstruction. However, this thermal treatent has been tested in several thesis in our research group, showing high reproducibility and successful results [145, 146].

At the end of the process, atomically flat surfaces with stair-like morphology are obtained. Under this procedure, the substrates are conditioned and it leads to a reproducible growth and excellent performances of the metallic perovskite oxide films.

\subsubsection{Growth and characterization of $\mathrm{La}_{1-\mathrm{x}} \mathrm{Sr}_{x} \mathrm{MnO}_{3}$ thin films}

Different strategies to achieve high quality epitaxial LSMO thin films can be obtained by either physical [147] or chemical methods[51, 148]. In this thesis, we have used this latter approach, the Chemical Solution Deposition (CSD) route to achieve highly-crystalline epitaxial samples. In the following, a brief summary of the growth methodology and the structural material characterization is presented.

\section{CSD route for LSMO growth}

The Chemical Solution Deposition (CSD) technique is a very versatile, low cost and scalable process for the growth of epitaxial thin films [149-151]. The CSD route can be employed to grow materials in a wide range of applications such as superconducting coated conductors [152], ferroelectricity [153] and ferromagnetic structures[154, 155].

The conventional CSD procedure is a multi-step deposition process. As depicted in figure 2.1, it consists of the preparation of a stoichiometric solution, its deposition on a single crystalline substrate which then goes through a thermal treatment which first decompose the organic species at lower temperatures and then induces the oxide crystallization at higher temperatures.

Several parameters should be taken into account to choose the adequate precursor and solvents, such as solubility, stability, ageing, molarity of the solution, its rheological properties and homogeneity after decomposition. For the 


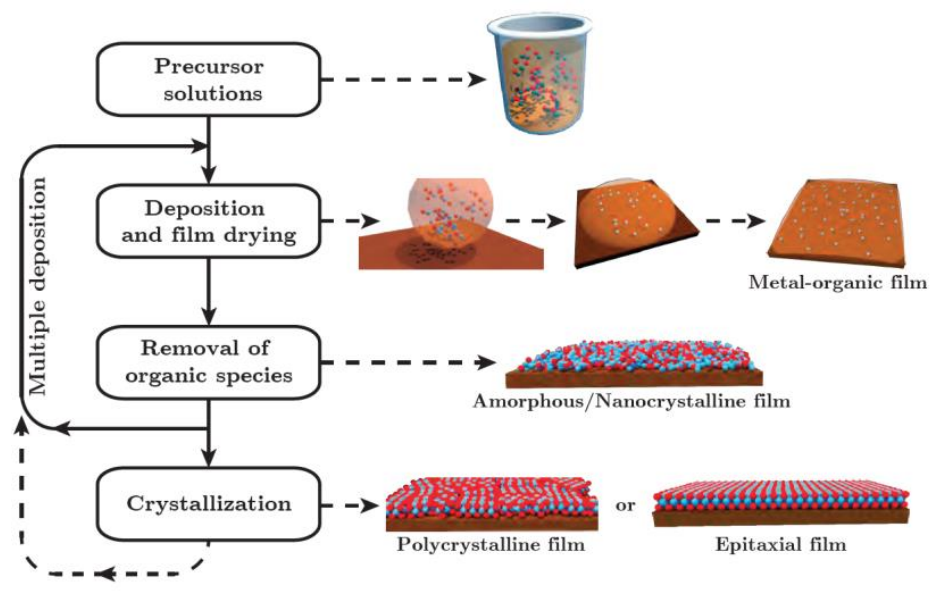

FIGURE 2.1: Steps involved in the CSD methodology.

growth of epitaxial $\mathrm{La}_{1-x} \mathrm{Sr}_{x} \mathrm{MnO}_{3}$ thin films, in this thesis, we have employed a precursor solution route that have been developed in our group [51, 145, 156]. It consists of mixing stoichiometric amounts of lanthanum (III) acetate hydrate $\left(\mathrm{La}\left[\mathrm{CH}_{3} \mathrm{COOO}\right]_{3} \cdot \mathrm{H}_{2} \mathrm{O}\right)$, strontium acetate $\left(\mathrm{Sr}\left[\mathrm{CH}_{3} \mathrm{COOO}\right]_{3}\right)$, and manganese (II) acetate $\left(\mathrm{Mn}\left[\mathrm{CH}_{3} \mathrm{COOO}_{2}\right)\right.$. The amount of each salt depends on the desired $\mathrm{Sr}$ content (x). In this thesis, we have grown films with three different Sr content: $x=0.3,0.2$ and 0.15 . These salts are dissolved in a mixture $4: 1$ of acetic acid and water. These products are commercially available and were supplied by Sigma Aldrich. The initial solution is then stirred up for $30 \mathrm{~min}$ at $50^{\circ} \mathrm{C}$ and afterwards, filtered with a $200 \mathrm{~nm}$ filter. This solution is stable for months and can be kept at room temperature.

Once the stoichiometric solution has been prepared with the desired Sr content and concentration, a volume of $15 \mu \mathrm{L}$ is deposited on a substrate and then spin-coated. The deposition is performed in a low humidity atmosphere $(<10 \%)$. The substrate reaches $6000 \mathrm{rpm}$ in 1s, and then this speed is kept for 2 minutes, as depicted in figure 2.2 (a). Then, the substrate is placed on a hot place at $70^{\circ} \mathrm{C}$ for 10 minutes to dry the excess of solvent. At the end of this process, the substrate is covered with an homogeneous gel layer. In CSD processes, the final thickness of the deposited film can be controlled with the angular velocity, spinning time and solution concentration and viscosity [150]. For all the films deposited on this thesis, identical volume, velocity, acceleration and spinning time has been employed. By keeping all these conditions constant, we expect that molarity will be the key parameter controlling the final thickness of the film. Figure 2.2 (b) shows the final thickness layer as a function of the molar concentration of the solution.

The desired LSMO epitaxial crystalline phase is achieved through a high temperature thermal treatment in a flowing oxygen atmosphere. The growth thermal profile (heating ramp, growth temperature, dwell time) has been optimized over the years by several people in our group to assure flat and homogeneous high quality films $[145,148]$.

The thermal profile consists of a heating ramp of $3^{\circ} \mathrm{C} \cdot \mathrm{min}^{-1}$ up to $1000^{\circ} \mathrm{C}$, where the LSMO phase is formed. At $700^{\circ} \mathrm{C}$, a constant $\mathrm{O}_{2}$ flow of $0.6 \mathrm{l} \cdot \mathrm{min}^{-1}$ is introduced in the chamber, and closed again at the same temperature during the cooling 
a)

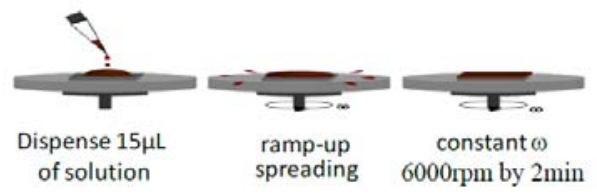

b)

FIGURE 2.2: (a) Diagram of the spin-coating process. (b) $\mathrm{La}_{0.8} \mathrm{Sr}_{0.2} \mathrm{MnO}_{3}$ film thickness for different molarity of the deposited solution.

ramp $\left(-3^{\circ} \mathrm{C} \cdot \mathrm{min}^{-1}\right)$. The dwell time at high temperature depends on the concentration of the solution: 5, 15 and $30 \mathrm{~min}$ for $0.05,0.1$ and $0.3 \mathrm{M}$ respectively. Figure 2.3 shows a summary of the thermal processes applied for the three concentrations.
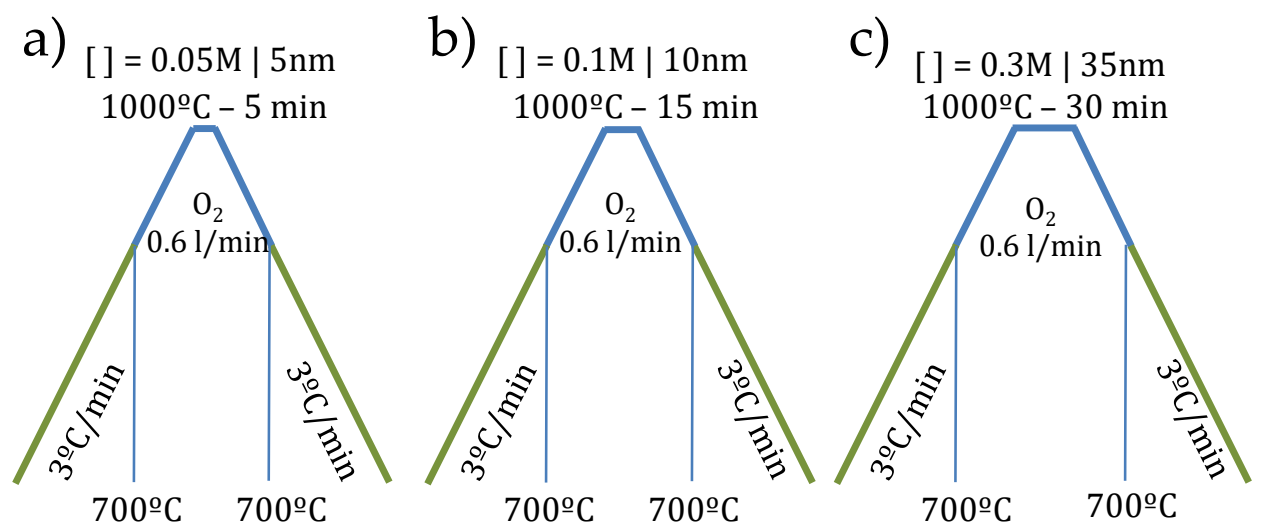

FIGURE 2.3: $\mathrm{La}_{1-\mathrm{x}} \mathrm{Sr}_{\mathrm{x}} \mathrm{MnO}_{3}$ for $x=0.2$ thermal growth process film for different molarity of the deposited solution. (a) $0.05 \mathrm{M}$. (b) $0.1 \mathrm{M}$.

(c) $0.3 \mathrm{M}$.

\section{Structural characterization of LSMO thin films}

To analyse the structure of $\mathrm{La}_{1-x} \mathrm{Sr}_{x} \mathrm{MnO}_{3}$ films we carried out X-ray diffraction (XRD) measurements. The thinner films $(t \leq 5 \mathrm{~nm})$ were not analysed since the equipment had not enough resolution to detect the LSMO Bragg peaks due to the low amount of material in the layer, which yields to a very low signal and to the overlapping of the STO substrate peaks with the corresponding (001) LSMO peaks [145], which masks the film contribution. The figure 2.4 (a) shows a $\theta-2 \theta$ scan from $20^{\circ}-80^{\circ}$ where the peaks of the STO substrate are strongly detected. In addition, shoulders corresponding to the (001) peaks of the $\mathrm{La}_{0.7} \mathrm{Sr}_{0.3} \mathrm{MnO}_{3}$ layers are observed. Figure 2.4 (b) shows a scan performed around the (003) reflection. The most intense reflections correspond to the $K_{\alpha_{1}}$ and $K_{\alpha_{2}}$ (003) STO substrate peaks. A shoulder indicating the (003) $\mathrm{La}_{0.7} \mathrm{Sr}_{0.3} \mathrm{MnO}_{3}$ reflection is detected around the value of $2 \theta=73.8^{\circ}$. Its intensity increases as the thickness of the $\mathrm{La}_{0.7} \mathrm{Sr}_{0.3} \mathrm{MnO}_{3}$ layer increases. 
a)

b)

FIGURE 2.4: a) $\theta-2 \theta$ scan performed from $20^{\circ}$ to $80^{\circ}$. Only peaks related to the STO and LSMO are found. b) XRD pattern of LSMO/STO with different thickness centered around the LSMO (003) reflection.The intensity of its shoulder is higher as the thickness increases.

In addition, Scanning Transmission Electron Microscopy (STEM) experiments were carried out in the group by Dr. Jaume Gazquez and Mr. Bernat Mundet. Figure 2.5 shows an STEM micrograph of an LSMO thin film grown by CSD-MOD [155]. The film shows highly crystalline and a cube-on-cube growth between the LSMO layer and the STO substrate. A sharp interface is developed with no trace of structural disorder such as misfit dislocations. These results indicate that the LSMO layers grow fully epitaxial ${ }^{1}$ on STO substrates and they are free of other possible organic contaminants.

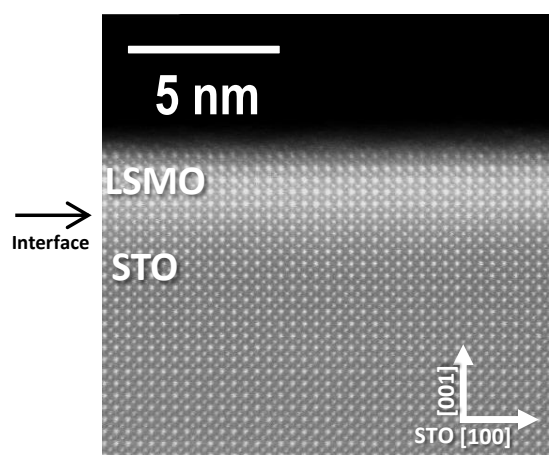

FIGURE 2.5: STEM image showing that the LSMO has grown fully epitaxial on STO with a sharp interface. Courtesy of Dr. J. Gazquez and Dr. J. Zabaleta [145]

\section{Morphological characterization of LSMO thin films}

Surface morphology of the films was studied by atomic force microscopy (AFM), as it will be detailed in next section. Figure 2.6 shows topography images of $5 \mathrm{~nm}$ (a), $10 \mathrm{~nm}$ (b) and $32 \mathrm{~nm}$ (c) LSMO thin films. For the thinner films (5 and $10 \mathrm{~nm}$ ) stair-like morphology of the substrate is followed by the film, whereas for the 30 $\mathrm{nm}$ sample a grain-like surface is observed. All the films present flat surfaces with low values of RMS, as indicated in the figure.

\footnotetext{
${ }^{1}$ Previous experience in the group showed strained films up to a critical thickness of $60 \mathrm{~nm}$.
} 
a)

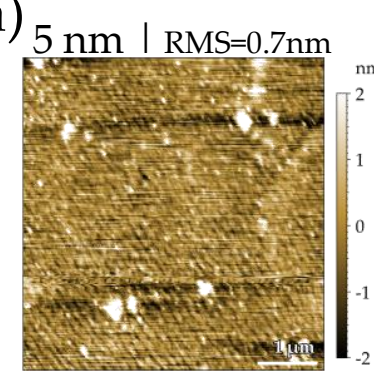

b)

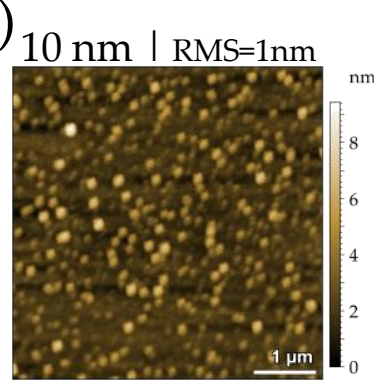

c)

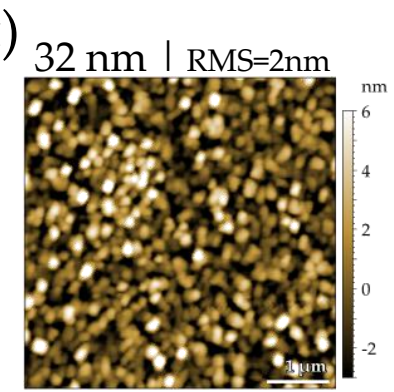

FIGURE 2.6: AFM topography images of (a) $5 \mathrm{~nm}$, (b) $10 \mathrm{~nm}$ and (c) $32 \mathrm{~nm} \mathrm{La} 0.7 \mathrm{Sr}_{0.3} \mathrm{MnO}_{3}$ samples. The RMS of the surfaces is indicated in each image.

\section{Physical properties of LSMO thin films}

The physical properties of our $\mathrm{La}_{1-x} \mathrm{Sr}_{x} \mathrm{MnO}_{3}$ thin films have been characterized by electrical transport properties and magnetization measurements. Both analysis have been performed by varying the Sr content of the films at constant thickness

\section{Transport properties of LSMO thin films}

As stated in the introductory chapter, the electrical conduction and the metalinsulator transition (MIT) in $\mathrm{La}_{1-x} \mathrm{Sr}_{x} \mathrm{MnO}_{3}$ thin films depends strongly on the $\mathrm{Sr}$ content. On the one hand, the substitution of Sr cations in La places affects the average cation radii, and therefore the Mn-O-Mn bond is changed. On the other hand, band filling $n=1-x$ is directly related to the Sr content. Although other factors such as biaxial strain, structural defects and oxygenation process can strongly influence the transport properties of the films, these are out of the scope of this thesis.

Resistivity curves, $\rho(T)$, were measured using the Van der Pauw method [157] in a commercial Physical Properties Measurement System (PPMS, Quantum Design) in the temperature range $400-10 \mathrm{~K}$. In figure 2.7 the resistivity plots of $10 \mathrm{~nm}$ $\mathrm{La}_{1-x} \mathrm{Sr}_{x} \mathrm{MnO}_{3}$ films with $x=\{0.3,0.2,0.15\}$ grown on STO substrates.

FIGURE 2.7: Resistivity as a function of temperature in $10 \mathrm{~nm}$ $\mathrm{La}_{1-\mathrm{x}} \mathrm{Sr}_{x} \mathrm{MnO}_{3}$ for different $\mathrm{Sr}$ content $\mathrm{x}$.

As reported for the bulk case [73], increasing the doping from $x=0.15$ to $x=0.3$ decreases the resistivity at RT due to the increases of charge carriers and double exchange mechanism enhancement. A clear shift of the MIT can be observed, with the highest value for $\mathrm{x}=0.3$. 


\section{Magnetic properties of LSMO thin films}

The exotic magnetic properties of manganites, in particular of $\mathrm{La}_{1-x} \mathrm{Sr}_{x} \mathrm{MnO}_{3}$ compounds, have been widely studied due to their magnetoresistive properties [73, 84, 147].In the case of epitaxial structures, a strain relaxation of lattice mismatch between the film and the substrate at the interface occurs, as the film thickness increases beyond a certain critical thickness of the film. Above this thickness, the film is relaxed and exhibits lattice constant similar to bulk sample. It has been reported that this strain may influence the physical properties by inducing a magnetic dead layer. The thickness of this layer depends a lot on the growth method employed [148, 151, 155, 158, 159]. To test the magnetic response of our films, magnetization curves were obtained for three $35 \mathrm{~nm} \mathrm{La}{ }_{1-x} \mathrm{Sr}_{x} \mathrm{MnO}_{3}$ films grown on STO substrates with different $\mathrm{Sr}$ content by applying a $0.5 \mathrm{~T}$ in-plane magnetic field to saturate the film while the temperature was varied between $5-400 K$, i.e., $\mathrm{M}(\mathrm{T})$ curves. The measurements were performed in a Superconducting Quantum Interference Device (SQUID). As shown in figure 2.8, the saturation magnetization decreases with $\mathrm{Sr}$ content. The ferromagnetic-paramagnetic transition temperature, the Curie temperature $\mathrm{T}_{c}$, also decreases with the doping concentration. This trend agrees with the literature [73]. In our films, all the $\mathrm{T}_{c}$ are found to be over $300 \mathrm{~K}$, which means that the layers remain ferromagnetic at room temperature for the range of Sr doping tested. In thin films, similar trend is observed. However, strain effects can largely affect the physical properties of the films [84].

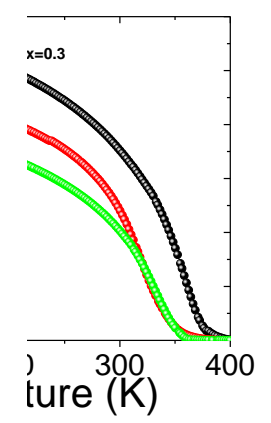

FIGURE 2.8: $\mathrm{M}(\mathrm{T})$ for $35 \mathrm{~nm}$ films different stoichiometry $(\mathrm{x})$ of $\mathrm{La}_{1-x} \mathrm{Sr}_{x} \mathrm{MnO}_{3}$

The growth method, crystal structure and thickness of the films can influence their final physical properties due to different impact in the strain microstructure. Deeper studies on the relation between microstructure of our films have been reported in refs. [51, 145].

\subsubsection{Growth and characterization of $\mathrm{RENiO}_{3}$ thin films}

Physical methods such as pulsed laser deposition and radio-frequency sputtering have been commonly used to obtain rare earth nickelates (RENO) [72, 78, 100, 160]. However, methods relying on chemical solutions have the advantage of giving a high level of control over the stoichiometry of the sample, besides being a low cost and scalable methodology. In this area, chemical vapour deposition has already been studied as an alternative $[97,161,162]$. However, the CSD approach has been scarcely explored to grow these materials [161]. 
The CSD-RENO methodology used in this thesis has been developed by other members of our group. The study and optimization of the growth process for different $R E$ cations was carried out by Mrs. Júlia Jareño. The full characterization of the resistive switching behaviour of the RENO thin films was carried out by me, mainly focused in $\mathrm{LaNiO}_{3}$ (LNO) and $\mathrm{NdNiO}_{3}$ (NNO) thin films.

In this section, the essential information about the growth process and structural characterization of our RENO thin films is presented.

\section{CSD route for RENO growth}

The CSD procedure presented for the LSMO case is applied for the growth of RENO thin films. In this case, commercially available (Sigma Aldrich) $\mathrm{Ni}\left(\mathrm{CH}_{3} \mathrm{COO}\right)_{2}$ and $\mathrm{La}\left(\mathrm{NO}_{3}\right)_{3}$ or $\mathrm{Nd}\left(\mathrm{NO}_{3}\right)_{3}$ salts are dissolved in stoichiometric proportion in methoxyethanol. The concentration of the solution determines the final thickness of the film, and a similar relation as the one in the LSMO case is obtained. The final solutions were stored under argon and remained stable for months.

The precursor solutions are then deposited on $\mathrm{LaAlO}_{3}$ and $\mathrm{SrTi} O_{3}$ single crystalline substrates by spin-coating. The same spinning and drying conditions after deposition than in the LSMO case are applied.

Then, the samples are placed on a quartz crucible inside a tubular furnace to perform a thermal treatment in oxygen atmosphere. For both LNO and NNO films, a heating ramp of $10^{\circ} \mathrm{C} \cdot \mathrm{min}^{-1}$ at $1 \mathrm{bar} \mathrm{O}_{2}$ atmosphere with a gas flow of $0.12 \mathrm{~L} \cdot \mathrm{min}^{-1}$ is set. The temperature and time of the dwell were varied for each $R E$ compositon. In the LNO case, the dwell is performed at $700^{\circ} \mathrm{C}$ for 1 hour whereas in the NNO case, the dwell is carried out at $900^{\circ} \mathrm{C}$ for 3 hours. In both cases, the cooling ramp is set at $-10^{\circ} \mathrm{C} \cdot \mathrm{min}^{-1}$. We used a lower dwell temperature in the LNO case because this oxide experiences a phase transition above $825^{\circ} \mathrm{C}$ [163].

Most of the studied films in chapter 4 were grown on LAO substrates. However, for characterization purposes, some results on STO substrates are commented here.

\section{Structural characterization of RENO thin films}

X-ray diffraction experiments were carried out in $25 \mathrm{~nm}$ samples. $2 \Theta$ scans for $\mathrm{LaNiO}_{3} / \mathrm{LAO}$ and $\mathrm{NdNiO}_{3} / \mathrm{STO}$ films, since the $\mathrm{LAO}$ peaks overlap with the NNO (001) reflections. The XRD data were acquired 20 and $55^{\circ}$, as shown in the figure 2.9 .

In both cases, only (001) reflections are observed. The peak detected in the LNO case at $\sim 36^{\circ}$ arises from an instrumental error, since no trace of secondary phases was detected in the 2D-XRD scans (not shown). LNO (100) reflections could be observed when grown either on LAO or STO substrates. However, for the NNO case, (100) reflections could only be observed on STO substrates due to the small mismatch with LAO and therefore, an overlapping of the peaks. However, epitaxial characterization was confirmed by TEM experiments.

In particular, Scanning Transmission Electron Microscopy (STEM) was used to analyze the structure of the films at the atomic level. STEM experiments were performed by Mr. Bernat Mundet and Dr. Jaume Gazquez. Figure 2.10 shows STEM 
a)

b)

FIGURE 2.9: XRD diffraction patterns of $25 \mathrm{~nm}$ LNO grown on LAO substrate (a) and NNO grown on STO substrate (b).

micrographs of LNO thin films of 12.5 and $25 \mathrm{~nm}$. Films show an homogeneous morphology and epitaxial growth. However, the presence of Ruddlesden-Popper Faults (RPF) [164] is also detected. This defect is schematically depicted in the right image of the figure. A RPF consist of the displacement of the lattice by half a unit cell in any of the two orthogonal directions eliminating a Ni plane. These defect allows the system to relax the strain generated during the growth process. Furthermore, the thicker the film and / or the more distorted perovskite structure $(\mathrm{Nd}>\mathrm{La})$, the bigger density of RPF in the films. First few unit cells remains defect-free and fully strained. From there RPF are present. With the displacement of half a unit cell, the distance between two La ions is larger than in the regular arrangement. In average, the cell parameter would be larger than expected because of the presence of the RPF. As it is shown in chapter 4, this defect plays a key role in the RS properties of our films. Therefore, more TEM micrographs with a more detailed explanation about the RPF is presented there.

a)

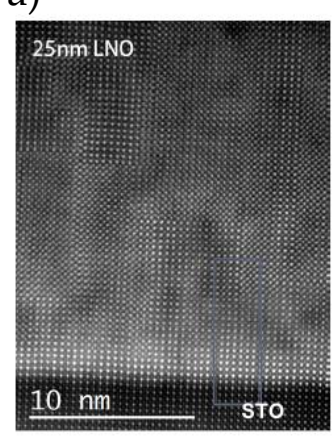

b)

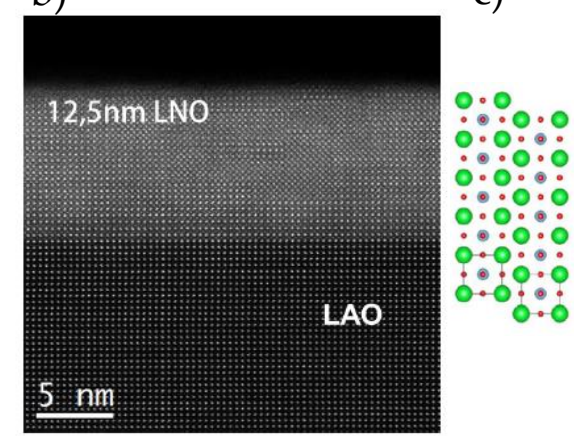

c)

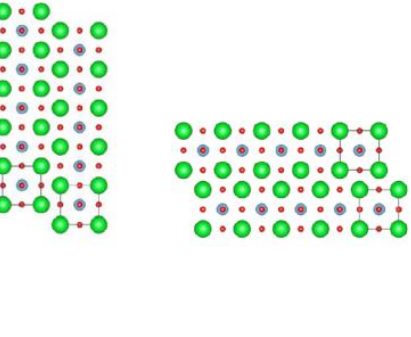

FIGURE 2.10: STEM micrographs of LNO thin films of (a) $25 \mathrm{~nm}$ and (b) $12.5 \mathrm{~nm}$. An schematic diagram of vertical and horizontal Ruddlesden-Popper Faults is shown in (c).

In summary, we confirm the epitaxial character of our RENO films, free of organic contaminants and thus their validity for further RS analysis.

\section{Morphological characterization of RENO thin films}

Surface morphology of the films was studied by atomic force microscopy (AFM). Figure 2.11 shows topography images of $25 \mathrm{~nm}$ LNO (a) and NNO (b) films. Both films are continuous with no pores and show grain structures of $\sim 20-50 \mathrm{~nm}$. 
LNO films present smaller roughness than the NNO films $(0.79 \mathrm{~nm}$ and $1.43 \mathrm{~nm}$, respectively).

a)

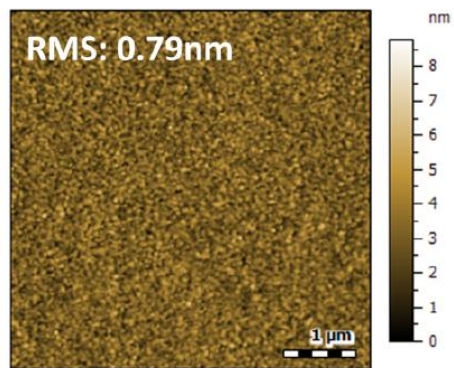

b)

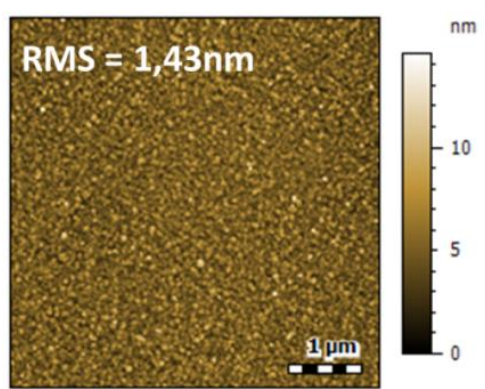

FIGURE 2.11: AFM topography images for LNO (a) and NNO (b) 25 $\mathrm{nm}$ thin films. RMS values are indicated in the images.

\section{Physical properties of RENO thin films}

The physical properties of our RENO films have been analysed through resistivity measurements as a function of temperature using the Van der Pauw configuration. In this way, the metal-insulator transition of these systems might be sensed. As stated in the introduction, RENO systems are charge-transfer insulators and metallicity is achieved by closing the gap via $\mathrm{O} 2 \mathrm{p}$ and $\mathrm{Ni} 3 \mathrm{~d}$ orbitals overlapping. The larger the bandwidth, the larger the overlap. Ni-O-Ni bonding angle plays a key role in the overlapping of the orbitals [77], and therefore, determining the MIT.

Figure 2.12 shows 6, 12.5 and $25 \mathrm{~nm}$ films of LNO (a) and NNO (b) grown on LAO substrates. In the LNO case, which presents the larger Ni-O-Ni overlapping among the $R E$ series, the metallic state is stabilized even for $6 \mathrm{~nm}$ film in the whole temperature range. In the NNO case, a metal-insulator transition is detected in the three films. The $25 \mathrm{~nm}$ film presents a transition temperature, $\mathrm{T}_{\mathrm{MIT}} \approx 190 \mathrm{~K}$, close to the value reported in bulk, $201 K$. However, decreasing the thickness of the NNO layer shifts the metal-insulator transition towards lower temperature. This fact is a reflect of the strong influence of the strain on the microstructure and on the physical properties. For instance, NNO samples grown on STO does not present MIT and remains insulating in our accessible temperature range (not shown).

a)

LNO b)

\section{NNO}

FIGURE 2.12: Resistivity measurements as a function of temperature for different thicknesses in LNO (a) and NNO (b) thin films grown on $\mathrm{LAO}$ substrates. 
In addition, the larger resistivity values upon thickness increase, observed in both LNO and NNO films, might be attributed to the higher density of RPF in the system. The disruption of the Ni-O-Ni bonds in the defect, where a Ni plane is removed and a shift of half a unit cell is found might strongly alter the resistivity of the material, and thus, to the resistive switching properties as well, as it is discussed in chapter 4.

\subsubsection{Growth and characterization of $\mathrm{YBa}_{2} \mathrm{Cu}_{3} \mathrm{O}_{7-\delta}$ thin films}

The growth of YBCO thin films by PLD has been widely studied for more than 20 years [165-167]. Huge efforts in the community were and are devoted to the optimization of growth process to enhance vortex pinning efficiency[168-170] and to scale the growth to km length for HTS 2-Generation coated conductors applications [114, 171, 172]. The CSD approach for YBCO growth is also largely studied, specially in our group [114, 125], but it will not be here reported. In this thesis, our effort was focused in achieving YBCO thin films with thickness ranging from 10 to $250 \mathrm{~nm}$ with high superconducting performance, low porosity and smooth surfaces.

Here, we have chosen the Pulsed Laser Deposition (PLD) [173, 174] technique to grow our YBCO thin films. Two reasons motivated us to do so. First, PLD samples present smoother surfaces than the equivalent CSD. Second, the growth of YBCO ultrathin samples by CSD requires a specific optimization process that was out of the scope of this thesis.

\section{PLD route for YBCO growth}

Pulsed laser deposition had several characteristics that made it remarkably competitive in the complex oxide thin-film research arena as compared to other film growth techniques. These principle attractive features are direct stoichiometric transfer, excited oxidizing species, and simplicity in initial setup and very smooth surfaces [175].

In essence, PLD is a physical vapour deposition process in which a pulsed laser is focused onto a target made out of the material to be deposited on a blank substrate. If the energy provided by the laser is high enough [176], each laser pulse ablates a small area of the material (typically few $\mathrm{mm}^{2}$ ) and creates a plasma plume directed to the substrate, which is placed $\mathrm{cm}$ away from the target. Usually laser pulses are short, in the order of ns. The created plasma plume presents the same composition as the target. Generally, a background gas is often introduced in the high-vacuum chamber for two reasons. First, multication thin-film often requires a reactive specie. In the case of oxide growth, flow of oxygen gas is introduced in the chamber during the deposition to ensure phase stability. Second, the background gas can be used to reduce the kinetic energy of the ablated species. Typical pressure ranges are in the order of 0.1-0.4 mbar. The particles from the plasma arrive to the surface of the substrate and rearrange minimizing their energy. This characteristics are resumed in figure 2.13. For a deeper understanding of the PLD process, the reader is referred to more specific and complete reviews [173, 174].

We employed the state-of-the-art PLD facilities at the Thin Film Laboratory at ICMAB. Several parameters were adjusted to optimize the growth process of the 


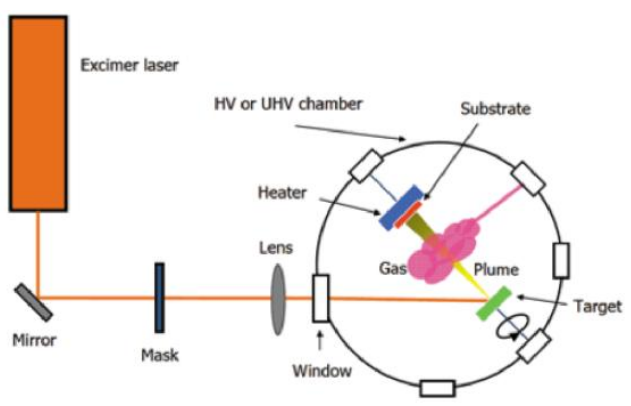

FIGURE 2.13: Scheme of a PLD process.

films in a PLD process. In our case, the YBCO were grown on STO substrates with the following parameters: The substrate was heated up to $\mathrm{T}_{\text {substrate }}=800-810^{\circ} \mathrm{C}$, with an $\mathrm{O}_{2}$ partial pressure of 0.3 mbar during the deposition and a fix targetsubstrate distance of $52.5 \mathrm{~mm}$. A high fluence laser $\left(\sim 2 \mathrm{~J} / \mathrm{cm}^{2}\right)$ working at a frequency of $10 \mathrm{~Hz}$ was used. During the cooling ramp, we increase the $\mathrm{P}\left(\mathrm{O}_{2}\right)$ in the chamber in order to obtain well oxygenated samples. A diagram of the process is depicted in figure 2.14.

a)

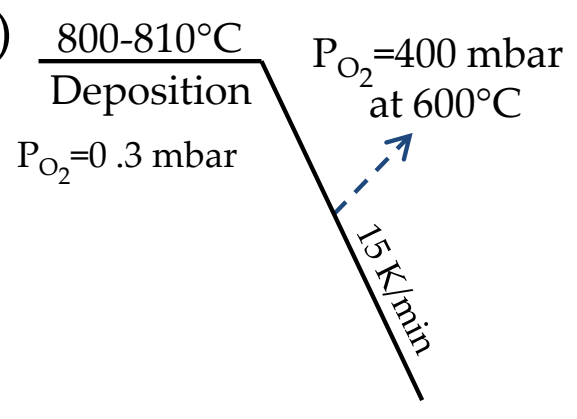

b)

FIGURE 2.14: Growth process profile (a) and thickness - number of pulses dependence.

The thickness of the film is mainly determined by the number of pulses. This parameter was varied from 200 to 4000 pulses to obtain samples ranging from 10 to $200 \mathrm{~nm}$. The thickness-pulses relation obtained is shown in figure 2.14.

\section{Structural characterization of YBCO thin films}

The structure of our YBCO films have been analysed by $\mathrm{x}$-ray diffraction techniques. The figure 2.15 shows a $\theta-2 \theta$ scan of a $200 \mathrm{~nm}$ YBCO sample grown on STO. Only peaks corresponding to the YBCO (001) reflections are observed. Neither secondary phases (such as $a b$-grains) nor polycrystalline phases are detected in the XRD pattern. The growth process described above leads to (001) epitaxial films with no secondary phases for all the thickness studied.

In addition, the microstructure of the film was studied by TEM. The figure 2.16 shows a micrograph of a $100 \mathrm{~nm}$ YBCO film grown on STO. It reveals high quality epitaxial films with sharp interfaces between the YBCO and the STO substrate.

Therefore, we validate our films for further characterization. The relation between the microstructure and the resistive switching properties is discussed in chapter 4 . 
FIGURE 2.15: $\theta-2 \theta$ scan of a YBCO $200 \mathrm{~nm}$ sample grown at $800^{\circ} \mathrm{C}$ and 0.3 mbar.

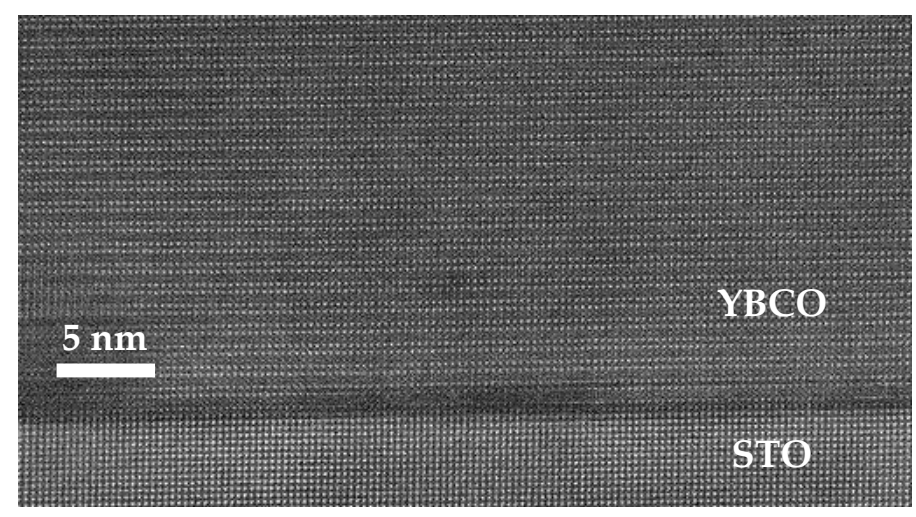

FIGURE 2.16: TEM image of a $100 \mathrm{~nm}$ YBCO film grown on STO. High quality crystalline films (001) epitaxially oriented are obtained

\section{Morphological characterization of YBCO thin films}

A microstructural characterization of the surface was performed by Scanning Electron Microscopy (SEM). The figure 2.17 shows three SEM micrographs for 22 (a), 50 (b) and $170 \mathrm{~nm}$ (c) YBCO films, respectively. In the thicker film, higher density of droplets and precipitates than in the thinner ones is detected. This particulates might be either ejected cluster particles from re-ablation in the target during deposition or aggregates formed during growth. In all the cases, smooth surfaces with no pores are observed.
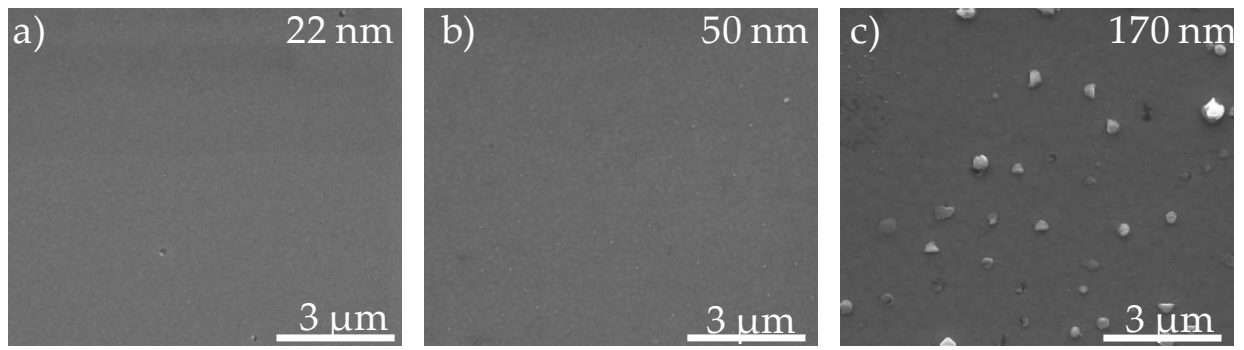

FIGURE 2.17: Surface morphology obtained by SEM for samples with different thickness: (a) 22, (b) 50 and (c) $170 \mathrm{~nm}$, respectively. 


\section{Physical properties of YBCO superconducting films}

The physical properties of the YBCO thin films have been characterized by electrical transport measurements and magnetic induction measurements. From these measurements, the superconducting transition and the critical current density of the films could be determined.

\section{Transport properties of YBCO thin films}

As stated in the introduction, $\mathrm{YBCO}$ is a superconducting material with a transition temperature $\mathrm{T}_{c} \approx 93 \mathrm{~K}$. The critical temperature of YBCO films with different thickness $(12-200 \mathrm{~nm})$ have been evaluated by resistivity measurements as a function of temperature in a 4-point configuration. The resistivity plots in the figure 2.18 correspond to several films with different thickness. All the films present a superconducting transition from a normal metallic state. For films thicker than 50$200 \mathrm{~nm}$, the resistivity value at room temperature is very close to the bulk value, $\rho_{300 \mathrm{~K}} \approx 300 \mu \Omega \cdot \mathrm{cm}^{-1}$ and $T_{c} \approx 92 \mathrm{~K}$. Below $50 \mathrm{~nm}$, a degradation of the superconducting properties is observed with an increase of one order of magnitude of the resistivity and a decrease of the $\mathrm{T}_{c}$ is found, being $\mathrm{T}_{c} \approx 60 \mathrm{~K}$ for the $12 \mathrm{~nm}$ film. Possible effects arising from strain might be at the origin of this phenomenon.

FIGURE 2.18: Resistivity measurements performed in a PPMS by varying the temperature from $300 \mathrm{~K}$ to $10 \mathrm{~K}$.

\section{Superconducting properties of YBCO thin films}

The superconducting performance of the films was investigated by performing SQUID magnetometry measurements on the films. The critical current density, $\mathrm{J}_{c}$, of the sample can be estimated from non-invasive magnetic induction measurements. To do so, the temperature is set to $5 \mathrm{~K}$ and then a high magnetic field is applied (3T) perpendicular to the film to assure that the whole sample is penetrated by magnetic flux lines. Then, the field is removed and the superconductor is taken to its remanent state. Then, the temperature is increased and the magnetization of the sample is recorded as a function of temperature. According to the Bean model $[177,178]$, we can obtain the critical current density $\left(\mathrm{J}_{c}\right)$ by using the following equation, valid for a square thin film.

$$
J_{c}=\frac{3 \Delta M}{a}=\frac{6 m}{a^{3} t}
$$


Where $t$ is the thickness, a the lateral size of the sample $(5 \mathrm{~mm}), \mathrm{M}$ is the saturated magnetization and $\mathrm{m}$ is the magnetic moment. The acquired $J_{c}(T)$ curves for YBCO films of different thickness are shown in figure 2.19 a). For all the films with $t>50 \mathrm{~nm}$, high values of critical current density are found. For lower thicknesses $(22 \mathrm{~nm})$, a remarkable decrease of the superconducting performance is foundi in agreement with the decrease in $\mathrm{T}_{c}$. However, even for this low thickness the film remains in the superconducting state at $77 \mathrm{~K}$. For clarification, values of $\mathrm{J}_{c}(77 \mathrm{~K})$ have been extracted and plotted separately as a function of thickness on figure $2.19 \mathrm{~b}$ ). High $\mathrm{J}_{c}$ values in the order of $2.5-3 \mathrm{MA} / \mathrm{cm}^{2}$ are obtain for $t>50 \mathrm{~nm}$, whereas $\mathrm{J}_{c}$ values strongly decrease for $t<50 \mathrm{~nm}$.

a)

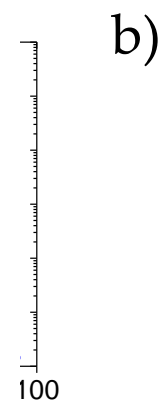

FIGURE 2.19: (a) Critical current density as a function of temperature for samples with different thickness grown on STO substrates.

(b) $\mathrm{J}_{c}(77 \mathrm{~K})$ and $\mathrm{J}_{c}(5 \mathrm{~K}) / 10$ values as a function of sample thickness.

To sum up, we have shown the excellent superconducting performance of YBCO thin films with $t>50 \mathrm{~nm}$, which present $T_{c} \approx 92 \mathrm{~K}$ close to the bulk value and high critical current density $\left(2.5-3 M A / \mathrm{cm}^{2}\right)$. For films of thickness below $50 \mathrm{~nm}$, the superconducting performance decreases. Despite this, these latter films present good enough superconducting characteristics to evaluate their resistive switching properties.

\subsubsection{Growth and characterization of $\mathrm{CeO}_{2} / \mathrm{LSMO}$ and $\mathrm{CeO}_{2} / \mathrm{YBCO}$ bi- layer systems}

The deposition of an ex-situ buffer layer on functional complex oxides (vacuum and non-vacuum techniques) remains challenging because it can induce structural and/or oxidation state modifications which could strongly affect the physical properties[179-181] of the initial film. During this thesis, we demonstrated that the $\mathrm{CeO}_{2}$ capping buffer layer deposited at low temperature by Atomic Layer Deposition (ALD) could be successfully integrated on functional complex oxides for RRAM applications [182].

The ALD deposition process was performed by Dr. Mariona Coll while the growth of the YBCO film and the structural and physical characterization of the bilayer was mainly performed by me. 


\section{ALD route for $\mathrm{CeO}_{2}$ growth}

Atomic Layer Deposition (ALD) has emerged as a powerful technique for the growth of thin films boosted by industry's interest due to its capability for atomic layer control and exceptional conformal coating on 3D high aspect ratio structures ([183], and references therein). In addition, ALD offers low working temperatures (even at room temperature) [184-186] and excellent uniformity at large scale. ALD is a well-established technique in several fields such as high- $\kappa$ dielectrics or DRAM [187]. Recently, ALD is being more and more used for RS applications [188-190].

ALD is a chemical gas-phase deposition technique where the film growth proceeds through a sequential self-limiting surface reaction achieved by alternating the precursor gases in the chamber. The fact that ALD is a self-limited process produces atomic level control of the film.

For the deposition of $\mathrm{CeO}_{2-\mathrm{x}}$, commercially available $\mathrm{Ce}(\text { thd })_{4}$ (thd $=$ tetrakis(2,2,6,6-tetramethyl-3,5-heptanedionato) (STREM) precursor and ozone $\left(\mathrm{O}_{3}\right)$ as the oxygen source at $275^{\circ} \mathrm{C}$. The final thickness of the $\mathrm{CeO}_{2-\mathrm{x}}$ film depends on the number of cycles that the reaction is repeated. In this thesis, the thickness of the film has ranged from 3 to $10 \mathrm{~nm}$. The films were deposited onto CSD-LSMO and PLD YBCO. In the following, the structural and physical characterization of the integrated bilayer film systems after the deposition of the ceria are presented.

\section{Structural characterization of $\mathrm{CeO}_{2}$ bilayer systems}

The structural characterization of the heterostructures have been performed through XRD measurements and Aberration corrected scanning transmission electron microscopy (STEM) imaging.

XRD $\theta-2 \theta$ scans of the YBCO films before and after ALD-CeO2 deposition are shown in figure 2.20 (a). (001) YBCO reflections are observed in both cases. In addition, after the deposition two extra peaks at $2 \theta=33^{\circ}$ and $2 \theta=69.4^{\circ}$ indicates the formation of epitaxial $\mathrm{CeO}_{2}$. The small lattice mismatch between $\mathrm{YBCO}$ and $\mathrm{CeO}_{2}(\epsilon=-0.52 \%)$ favours the epitaxial growth, even though a fluorite structure must adapt to a perovskite through a $45^{\circ}$ growth rotation. The presence of no additional peaks suggests that no interfacial reaction is taking place between YBCO and $\mathrm{CeO}_{2}$. This will be also supported by the analysis of the physical properties of the films, presented below. The same characteristics are observed in the LSMO case (not shown).

Figure 2.20 (b) shows a high resolution Z-contrast image of the $\mathrm{CeO}_{2} / \mathrm{LSMO}$ bilayer with sharp interfaces between firstly, LSMO and STO and secondly the LSMO layer and the ceria. Fully continuous and dense $\mathrm{CeO}_{2}$ layer is observed with an uniform thickness distribution in the whole sample. Poor epitaxy is observed above a couple of atomic layers. Since $\mathrm{CeO}_{2-x}$ epitaxial growth has been achieved on YSZ single crystal substrates [185], which presents a much higher lattice mismatch with $\mathrm{CeO}_{2}(\epsilon=-4.8 \%)$ than LSMO $(\epsilon=+1.21 \%)$, the poor epitaxy observed might be attributed to the low growth temperature employed. According to the experience in the group, fully epitaxial quality of ALD-CeO $\mathrm{C}_{2}$ on $\mathrm{LSMO}$ could be obtained by optimizing the ALD process growth temperature and also by performing high temperature post annealing treatments. Since the $\mathrm{CeO}_{2-x}$ layer will be just used as a protective layer and oxygen reservoir, we did not need to optimize its epitaxy. 
a)

b)

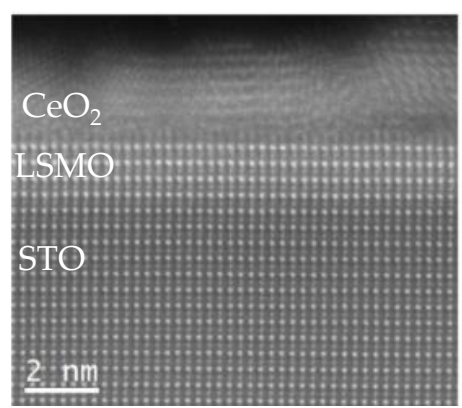

FIGURE 2.20: (a) XRD $\theta-2 \theta$ scans on YBCO/STO before and after the deposition of ALD-CeO 2 . (b) STEM Z-contrast image of 2 nm ALD-CeO2 films grown on CSD-LSMO/STO substrate viewed along [110] zone axis.

\section{Morphological characterization of $\mathrm{CeO}_{2}$ bilayer systems}

The surface morphology of $2 \mathrm{~nm}$ CSD-LSMO and $20 \mathrm{~nm}$ PLD-YBCO films before and after the deposition of 2-3 nm ALD-CeO ${ }_{2-x}$ films is investigated by AFM. Figure $2.21 \mathrm{a}$ ) and b) correspond to the LSMO case, whereas c) and d) to the YBCO film, as indicated in the insets of the images. In both cases, the surface morphology is not modified by the deposition of the $\mathrm{CeO}_{2}$ and their rms value before and after the deposition is preserved, $0.7 \mathrm{~nm}$ for LSMO and $0.6 \mathrm{~nm}$ for YBCO.
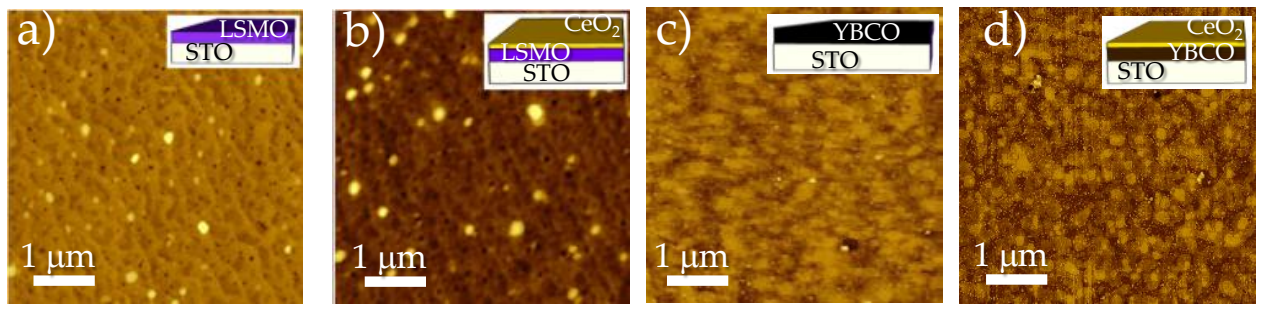

FIGURE 2.21: AFM topographic images of $2 \mathrm{~nm}$ CSD-LSMO/STO (a), $2 \mathrm{~nm}$ ALD-CeO $2 / 2$ nm CSD-LSMO/STO (b), 2 nm PLD-YBCO (c) and $2 \mathrm{~nm} \mathrm{ALD-CeO} / 20 \mathrm{~nm}$ PLD-YBCO (d) systems. In both cases, the RMS values are preserved after the deposition ( $\mathrm{rms}=0.7$ and 0.6 for LSMO and YBCO, respectiveli). The z-scale of the topographic images is $8 \mathrm{~nm}$.

\section{Physical properties of $\mathrm{CeO}_{2}$ bilayer systems}

The effect of the ALD- $\mathrm{CeO}_{2-x}$ deposition process of on the physical properties of the YBCO and LSMO films, was evaluated by means of macroscopic magnetic properties before and after the deposition.

In particular, SQUID magnetic induction measurements in a $5 \mathrm{~nm} \mathrm{ALD-CeO} / 200$ $\mathrm{nm} \mathrm{YBCO}$ are shown in figure $2.22 \mathrm{a}$ ). We depict the critical current density as a function of temperature $J_{c}(T)$ of the bilayer with and without the $\mathrm{CeO}_{2-x}$ layer. It is remarkable that the deposition process does not degrade the YBCO layer showing almost the same the critical current density dependence with temperature.

In the LSMO case, the magnetization was measured as a function of temperature in an applied in-plane magnetic field of $0.5 \mathrm{~T}$ as shown in figure $2.22 \mathrm{~b}$ ) for a $3 \mathrm{~nm}$ 
$\mathrm{CeO}_{2} / 3 \mathrm{~nm} \mathrm{LSMO}$ and $10 \mathrm{~nm} \mathrm{CeO} / 10 \mathrm{~nm}$ LSMO on STO before and after the deposition. No change is observed between the as-grown LSMO/STO state and the bilayers $\mathrm{CeO}_{2} / \mathrm{LSMO} / \mathrm{STO}$ after the deposition process.
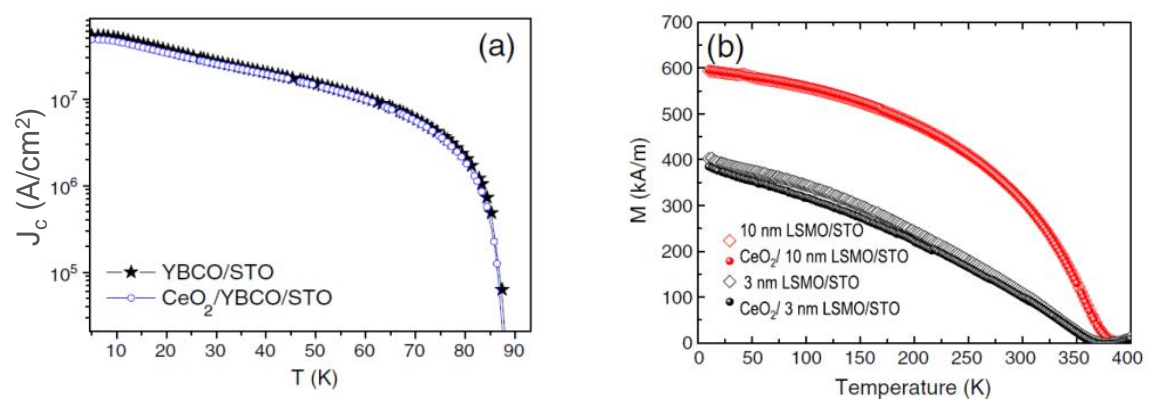

FIGURE 2.22: (a) Critical current density of a $200 \mathrm{~nm}$ YBCO film before and after depositing $5 \mathrm{~nm}$ ALD-CeO 2 (b) Temperature dependence of the saturation magnetization at $0.5 \mathrm{~T}$ for LSMO/STO thin films before and after ALD-CeO2.

Thus, we have validated the ALD-CeO ${ }_{2}$ ex-situ integration approach and demonstrated that the original performance of the metallic perovskite films remains unaffected by the ALD process.

\subsubsection{Metal deposition and device fabrication}

Along this thesis, metallic electrodes were required for either 4-point configuration measurements (LSMO, YBCO), to be used as a guide reference within the sample, or to study the resistive switching effect in encapsulated $\mathrm{Ag} / \mathrm{CeO}_{2} / \mathrm{LSMO}$ heterostructures, as it will be shown in chapters 3 and 4 . In some cases, lithography processes will be required to defined confined bridges. In this section, we present the photolithography process, etching techniques and metal evaporation methodologies used during this thesis. The optimization of these processes have been performed in our group along the years. All of them were performed in the clean room facilities at the Institut de Ciència de Materials de Barcelona (ICMABCSIC).

\section{Photolithography}

Lithography is a technique used to transfer a pattern onto a substrate/film. This pattern is subsequently used to etch an underlying thin film. In particular, photolithography refers to one kind of lithography that uses a light source (ultraviolet (UV)) to define the desired pattern that is transferred into a substrate/film. The procedure to perform a photolithography process in our perovskite films is as follows:

Pattern design: With an specific software (CleWin 5), the layout that should be transferred into the film is created at will. In our setup, this layout can be easily modified, thus adapting it to the specific sample and experiment requirements.

Photoresist deposition: The photoresist is a polymeric photosensitive material which can be deposited by spin-coating onto the sample in liquid form. The 
spinning speed and photoresist viscosity will determine the final resist thickness. In here, we have used the so called positive photoresist, which makes the UV-exposed areas to be dissolved in the subsequent development stage. In particular, we have employed positive photoresist MOCROPOSIT S181342. It is deposited by spin-coating at 5000rpm for 20 seconds, obtaining $\sim 1 \mu \mathrm{m}$. After the spinning of the resist, it is soft-baked at $90^{\circ} \mathrm{C}$ for $1 \mathrm{~min}$ in a hot plate in order to remove the solvents from the resist and improve adhesion.

UV exposure: Then, the exposure of the sample is performed in a Micro Writer ML by Durham Magneto Optics through a direct-write lithography process [191]. The sample is placed in a chamber where an autofocus correction and alignment is performed prior the exposure. Once these tasks have been successfully completed, the layout is loaded through software, specifying the dose. Typical dose values for our metallic perovskite films are between $150-250 \mathrm{~mJ} / \mathrm{cm}^{2}$ using a UV-laser of $1 \mu \mathrm{m}$. The time required to carry out this step is around 30-60 $\mathrm{min}$.

Photoresist development: After the exposure, the photoresist is developed in a solution composed of water $98 \%$ and tetramethyl ammonium $2 \%$ MICROPOSIT MF-319 for 45seg. The exposed areas are dissolved, leaving trenches in the photoresist, where the sample surface is ready for either metal deposition or etching process. At the same time, the undeveloped areas act as a protection where metal deposition or etching is undesirable.

Metallization or etching step: Metal deposition through DC sputtering or film etching by either physical or chemical methods in the developed areas is carried out at this stage. These techniques are explained in detail in following subsection. After these processes, The unexposed photoresist and uncovered metal areas (if any) are removed by rinsing the sample in acetone.

Figure 2.23 shows schematically all the steps involved in the described process.

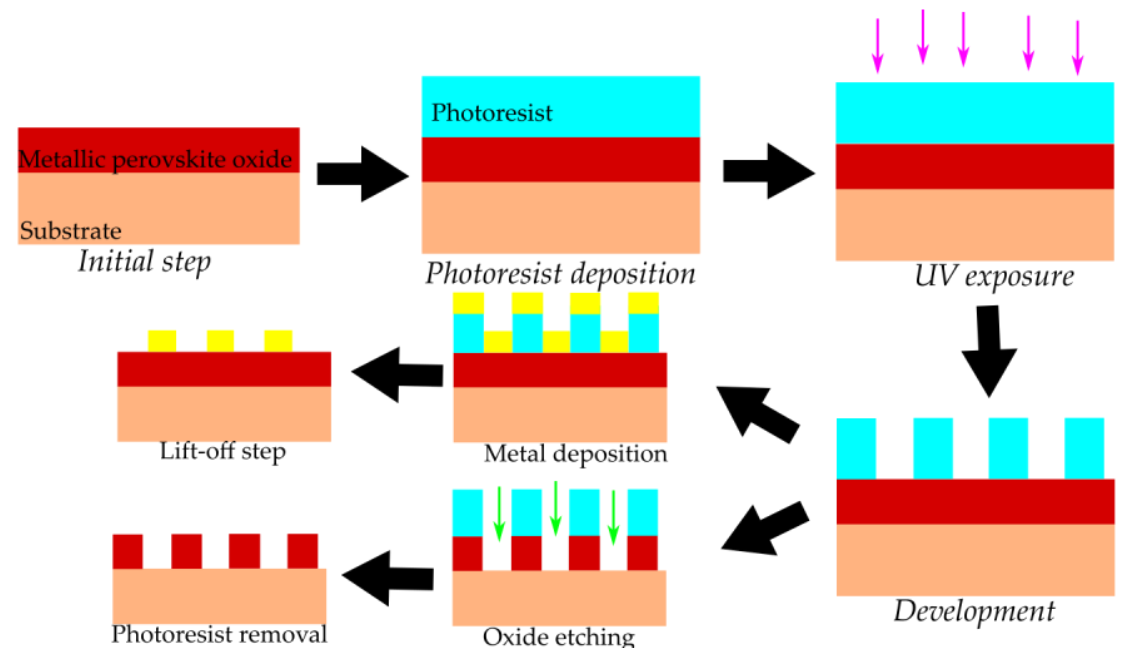

FIGURE 2.23: Photolithography process followed in this thesis.

\section{Metal deposition and lift-off}

In this thesis, we have used DC sputtering to deposit Ag and Au metal electrodes in our metallic perovskite films. In sputtering [192], a target made out of the metal 
to be deposited is bombarded with high-energy inert Ar ions in plasma ambient. As a result, individual atoms or clusters are removed from the surface and ejected towards the film. The Ar ions bombarding the target are produced in direct current plasma.

A sputtering system from Twente Solid State Technology (TSST) is used to sputter $\mathrm{Au}$ or Ag metal electrodes. The patterned structures range from 10 to $500 \mu \mathrm{m}$ in size, depending on each experiment, as it will be shown in the following chapters. Each metal has been deposited with the conditions defined in table 2.2.

\begin{tabular}{|l|l|l|l|l|l|l|l|}
\hline Metal & $\begin{array}{l}\text { Vacuum } \\
(\mathrm{mbar})\end{array}$ & $\begin{array}{l}\text { Ar } \\
\text { flux } \\
(\mathrm{sscm})\end{array}$ & $\begin{array}{l}\text { Gas } \\
\text { pres- } \\
\text { sure } \\
(\mathrm{mbar})\end{array}$ & $\begin{array}{l}\text { Voltage } \\
(\mathrm{V})\end{array}$ & $\begin{array}{l}\text { Current } \\
(\mathrm{A})\end{array}$ & $\begin{array}{l}\text { Time } \\
(\mathrm{min})\end{array}$ & $\begin{array}{l}\text { Thick- } \\
\text { ness } \\
(\mathrm{nm})\end{array}$ \\
\hline $\mathrm{Au}$ & $10^{-6}$ & 20 & 0.05 & 425 & 0.09 & 5 & 50 \\
\hline $\mathrm{Ag}$ & $10^{-6}$ & 20 & 0.05 & 500 & 0.08 & 10 & 50 \\
\hline
\end{tabular}

TABLE 2.2: Conditions employed for Au and Ag sputtering deposition.

The final structures are attained by a lift-off process in an acetone bath, where all unexposed photoresist including the overlying metal coating is removed. In all cases, the sputtered metals present a very good adhesion to our metallic perovskite oxides, without the need to use an adhesive layer. In addition, the contact resistance has been demonstrated to be low enough avoiding the need of post annealing process [156]. Typical values of the resistances measured in $\mathrm{Au}$ and Ag sputtered samples are 800 and $600 \Omega$, respectively, which leads to contact resistance values of $\sim 10^{-2} \Omega \cdot \mathrm{cm}^{2}$. In the case of superconducting transport measurements, a lower contact resistance is required and then a post annealing process of the metal contacts was carried out at $450^{\circ}$ for $1 \mathrm{~h}$ in oxygen atmosphere to decrease the contact resistance to a $10^{-8} \Omega \cdot \mathrm{cm}^{2}$.

\section{Oxide etching}

Etching is used to remove material in a selective way in order to create patterns. In our case, the pattern is previously defined by the photolithography process. The developed resist leads to unmasked areas that can be removed either by wet (chemical) or dry (physical) etching.

Wet etching consists of a liquid chemical attack to remove the material. It is strongly isotropic which limits its application for high-resolution patterning. In dry etching, plasmas or etchant gasses produce the material removal. It is highly anisotropic and therefore it is more capable for transferring small structures. However, the remaining material might be damaged during the process due to deoxygenation of the film. In this work, since we do not need to transfer sub-micron features and good performance of the films should be preserved, we have employed wet etching to pattern both YBCO and LSMO thin films.

In the LSMO, the wet etching is performed using a $0.2 \%$ diluted piranha solution $\left(\mathrm{H}_{2} \mathrm{SO}_{4}+\mathrm{H}_{2} \mathrm{O}_{2}+\mathrm{H}_{2} \mathrm{O}\right)$. An etching rate of $10-20 \mathrm{~nm} / \mathrm{min}$ is obtained. 
In the YBCO case, the wet etching is carried out by immersing the film in an acid solution of $0.1 \% \mathrm{H}_{3} \mathrm{PO}_{4}$. This solution leads to an etching rate of $100 \mathrm{~nm} / \mathrm{min}$ approximately.

\subsection{Characterization techniques}

The aim of this section is to provide an overview of the most relevant techniques employed in this thesis to investigate the RS effect. These techniques are atomic force microscopy, scanning tunnelling microscopy and probe station measurements equipped with external sources analysers. Special emphasis is put in those parts where I had a significant contribution to develop the experimental setup and measurement procedure. At the end of this chapter, a list of other characterization techniques employed in this thesis and my implication in each one is also mentioned. A short description of these techniques can be found in Appendix A.

Scanning Tunnelling Microscopy (STM) was the first breakthrough in the so called scanning probe techniques in 1981, invented by Heinrich Rohrer and Gerd Binnig [193], at that time, working at IBM Zürich. Its working principle is illustrated in further sections. This invention immediately triggered the development of other local probe techniques able to sense at the nanometer scale different physicochemical surface properties of the specimen under test, giving birth to the so called Scanning Probe Microscopies (SPM). The first big breakthrough was made in 1986 with the invention of the Atomic Force Microscope (AFM) by Binning, Gerber and Quate[194]. This instrument overcame some of the limitations of the STM, and will be pointed out in next sections.

In general terms, in any SPM technique, a short-range interaction is sensed by a local probe which is scanned over the surface of the specimen [195]. The magnitude under study is measured, recorded and then digitally processed. In these section, special emphasis will be made on the regular STM operated in air and AFM measurements in mode Conductive-Atomic Force Microscopy. Some outstanding applications of scanning probe techniques have been collected here [196, 197].

\subsubsection{Scanning Tunnelling Microscopy}

The STM technique is based on the quantum tunnelling effect. When two metals are sandwiched between a thin insulating barrier and an electrical potential is applied, electrons should flow from one metal to the other according to the rules of classical physics only if the applied potential is higher than the barrier. Conversely, quantum mechanics allow this process to happen even when the potential is lower than the barrier. In the particular case of the STM, an atomically sharp tip approaches an atomically flat surface, with a dielectric barrier in between. Usually this barrier is vacuum, but not only. It is also possible to measure in liquids[198], different gas atmospheres[199] or air [200]. If the distance between tip and surface is small enough (1-50 $\mathrm{A})$, their wave functions will overlap and therefore there is a finite probability of electron hopping between tip and sample, giving rise to quantum tunnelling, as shown in figure 2.24. The critical factor which gives this microscope extremely high vertical resolution (of few hundredths of an angstrom) is that the tunnel current flow between tip and sample depends exponentially on 
their relative distance. For a typical metal, the current will change by an order of magnitude for every $\AA$ in distance with an exponential decay as shown in equation 2.2.

$$
I \sim e^{2 \kappa d}, \kappa=\sqrt{\frac{2 m \phi}{\hbar}} \approx 0.5 \sqrt{\phi(e V)}^{-1}
$$

The lateral resolution depends on the apex geometry and the electronic orbitals of the tip. Generally, the electrons of the tip are confined to a very narrow channel allowing real-space imaging down to atomic scale.

The tunnelling regime is characterized by three interdependent parameters: The tip-sample distance (typically 5-10 $\AA$ ), the tunnelling current $\mathrm{I}_{t}$ (typically 0.01 - 10 $\mathrm{nA}$ ) and the bias voltage $\mathrm{V}_{t}$ (typically $0.01-2 \mathrm{~V}$ ). $\mathrm{I}_{t}$ and $\mathrm{V}_{t}$ are generally chosen to set a tunnel resistance $\mathrm{R}_{t}=\mathrm{V}_{t} / \mathrm{I}_{t}$ in the $G \Omega$ regime.
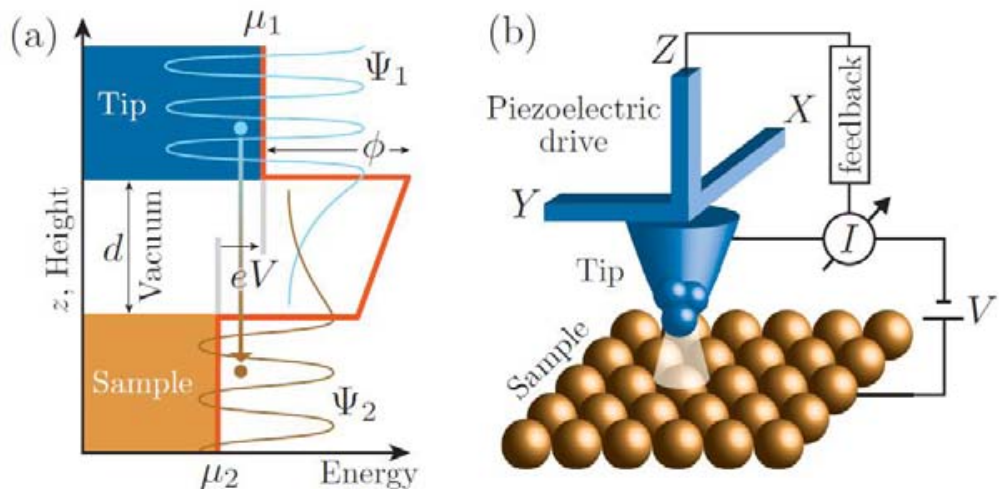

FIGURE 2.24: (a) Tunnelling process between the tip and sample across a vacuum barrier of width $d$ and height $\phi$. (b) Schematic view of an STM.

A scanning tunnelling microscope is capable to collect topography and spectroscopy data at local scales. In the topographic mode, the surface is mapped through the dependency of the tunnelling current, $\mathrm{I}_{t}$, upon the tip-to-sample distance. In the spectroscopy mode, the Local Density of states (LDOS) of the material is extracted through measurements of the tunnelling conductance, as it is explained below. However, the measured $\mathrm{I}_{t}$ corresponds to a convolution of the LDOS of the tip and the sample. To study intrinsic properties of the material, a tip with featureless DOS and well-defined Fermi energy $\left(\mathrm{E}_{f}\right)$ surface, (ideally spherical) should be used. The most common metals used for the tips are Au, W, Ir and PtIr.

\section{Operation modes}

To acquire topography images, two main modes are used. The first one is the so called constant-current imaging mode, which is the most used method to collect topographic maps. In this mode, $I_{t}$ is kept constant by adjusting with the feedback the tip vertical position during the scan. Since the tunnelling current integrates over all the states above or below the Fermi energy $E_{f}$ (depending on the polarity of the applied bias), the constant-current map represents a profile of constant integrated density of states (DOS). If the LDOS is homogeneous over the mapped area, this profile would correspond to a constant tip-to-sample distance, and therefore, 
recording the height of the tip as a function of the $(x, y)$ position a three-dimensional map of the surface is obtained.

The second mode is the so called constant-height mode. In this mode, the feedback loop is turned off and the the tip-sample distance is kept at a constant absolute value while mapping. The modulations in the $\mathrm{I}_{t}$ are produced then by a change in the tip-sample distance due to the surface corrugations. Therefore, recording the $\mathrm{I}_{t}$ as a function of position will directly reflect surface topography. This mode allows faster scanning than the constant-current mode. However, it is limited to surface with corrugations in the angstrom regime to avoid tip collision with the surface.

\section{Scanning Tunneling Spectroscopy}

Besides obtaining surface topography images, the LDOS of the surface can be obtained by performing scanning tunnelling spectroscopy (STS). In this mode, the feedback loop is turned off and the distance between tip and sample is kept constant. Then a voltage sweep is performed and the tunnelling current as a function of bias voltage is recorded. The differential of this tunnelling current $(\mathrm{dI} / \mathrm{dV}(\mathrm{V}))$ is proportional to the LDOS.

In first approach, the current between tip and sample can be expressed as

$$
I=\text { constant } \cdot \int d \omega[f(\omega-e V)-f(\omega)] N_{\text {tip }}(\omega-e V) N_{\text {sample }}(\omega, x)
$$

Where $\omega$ is the energy, $f(\omega)$ denotes the Fermi function, $N(\omega)$ is the DOS of tip and sample, and $x$ denotes the lateral position of the tip. If a $\mathrm{N}_{\text {tip }}$ is considered constant and taking the derivative with respect to the $\mathrm{V}$, the local conductance is obtained:

$$
G(V, x)=\frac{d I(V)}{d V} \propto \int d(\omega)\left[-f^{\prime}(\omega-e V)\right] N_{\text {sample }}(\omega, x)
$$

At $T=0, f(\omega)$ becomes a step function and then the conductance in eq. 2.4 becomes

$$
G=\frac{d I}{d V} \propto N_{\text {sample }}(e V, x)
$$

At higher temperatures, $f^{\prime}(\omega)$ becomes broader and therefore the features in the DOS of the sample are smeared out. Therefore, by taking the derivative of the I(V) spectra with respect to the voltage, the LDOS of the material at a specific location is obtained with a good approximation, using this method.

Most STS experiments use the so called current-imaging tunneling spectroscopy (CITS) firstly introduced by Hamers et al. in 1986 [201]. A CITS image consists of a regular matrix of points distributed over the surface. The tip scans over the surface with a fixed tunnelling resistance $\mathrm{R}_{t}=\mathrm{V}_{t} / \mathrm{I}_{t}$, recording the topographic profiles. At each point of the CITS array, the scan is frozen and the feedback turned off. With the tip $(x, y, z)$ position frozen, the voltage is swept to measure $\mathrm{I}(\mathrm{V})$ (or $\mathrm{dI} / \mathrm{dV}$ curves with a lock-in amplifier). After the sweep, the bias voltage is set back to $V_{t}$ and the scanning resumed. At the end of the experiment, a topographic image obtained at $\mathrm{V}_{t}$ and simultaneously obtained spectroscopic images reconstructed from the $\mathrm{I}(\mathrm{V})$ (or $\mathrm{dI} / \mathrm{dV}$ ) data. Since the feedback loop is turned off while sweeping, V can take 
any value, specially $V=0 \mathrm{~V}$. In that case, $G(0 \mathrm{~V})$ is called the Zero Bias Conductance $(\mathrm{ZBC})$, which is related to the number states available in the vicinity of the Fermi level.

\section{STM protocol measurement}

STM measurements were performed in Prof. Christoph Renner's group, under the supervision of Dr. Ivan Maggio-Aprile at the University of Geneva (Switzerland). I could perform these measurements thanks to two stays (May-July 2015 and MayJuly 2016) that were carried out during my PhD funded by the Spanish Ministry of Economy. A schematic diagram of the experimental setup and a picture of the microscope employed is shown in figure 2.25.
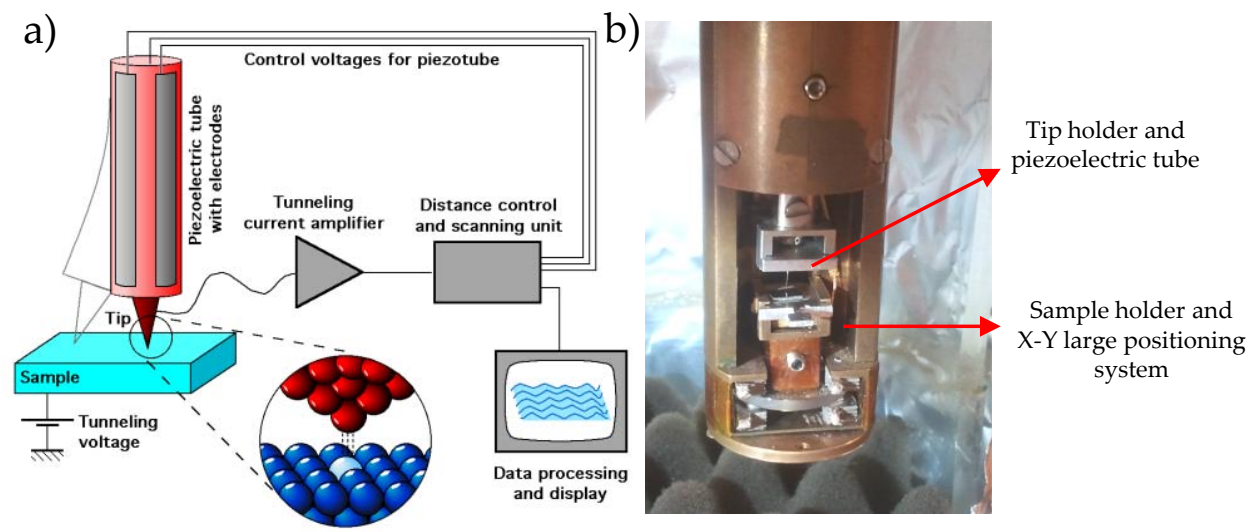

FIGURE 2.25: (a) Schematic representation of an STM imaging process (taken from [202]). (b) Microscope used in all the experiments at the University of Geneva.

The setup consist of a home-made STM controlled by a Nanonis SPM controller. Sample and tip holders are electrically isolated, and are located in a copper Faraday box hanging with a spring from another Faraday box on top a granite table. This configuration minimizes mechanical and electrical noise to a background noise of $\sim 200 \mathrm{fA}$. The tip holder is connected to the feedback system explained above. Tip motion is controlled by a piezoelectric tube with a working range of $20 \mu \mathrm{m} \times 20 \mu \mathrm{m}$ in the $\mathrm{XY}$ direction and $3 \mu \mathrm{m}$ in the $\mathrm{Z}$ component. Tip is grounded while the bias voltage is applied to the sample, which is firmly attached and electrically contacted to the holder with conductive silver paste. With this configuration, when applying a positive (negative) bias voltage $\mathrm{V}$ to the sample, electrons tunnel preferentially from the tip into unoccupied (occupied) sample states [202]. Additionally, this STM system has an optical microscope attached to facilitate the location of the tip onto specific areas within the sample.

For all the experiments shown in this Thesis, the same measurement conditions were used. A $\mathrm{V}_{\text {bias }}=800 \mathrm{mV}$ and a set-point current $\mathrm{I}_{t}=200 \mathrm{pA}$ were used. Topography images were acquired in a constant-current mode and spectroscopic data were acquired according to the CITS method explained above [201]. Figure 2.26 (a) shows a typical topography image in a $8 \mathrm{~nm} \mathrm{La}_{0.8} \mathrm{Sr}_{0.2} \mathrm{MnO}_{3}$ film. A stair-like terraces morphology is observed. The white line in the map corresponds to the profile in the figure $2.26(\mathrm{~b})$, where 1 u.c. $(\sim 0.4 \mathrm{~nm})$ steps are found. 


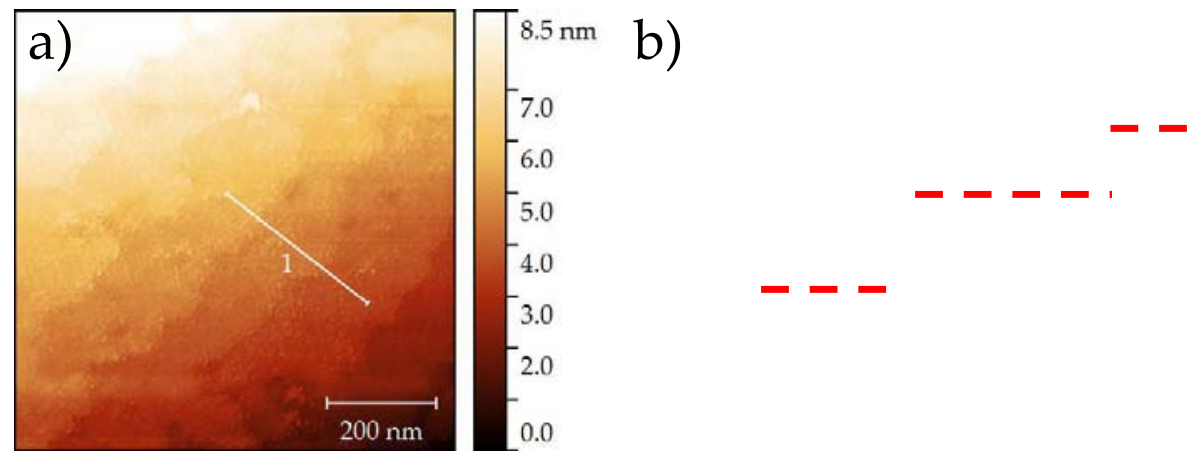

FIGURE 2.26: (a) $800 \times 800 \mathrm{~nm}^{2}$ topography image obtained for a 8 $\mathrm{nm}$ LSMO sample at $800 \mathrm{mV}$ and $200 \mathrm{pA}$. Stair-like terraces are observed. White line indicates the region where the profile in (b) was extracted.

For the spectroscopic measurements, the CITS method was used. The feedback in the $\mathrm{I}_{t}$ is turned off and $\mathrm{V}_{\text {bias }}$ is varied from -800 to $800 \mathrm{mV}$ to acquire $\mathrm{I}_{t}(\mathrm{~V})$ curves. Figure 2.27 (a) shows the typical I-V curve obtained in the same film, whereas (b) shows the so called conductance curve, $G(V)$. This $G(V)$ is obtained through the numerical derivative of the $\mathrm{I}(\mathrm{V})$ data.

a)

b)

FIGURE 2.27: (a) Typical shape of an I-V curve obtained in LSMO thin films. (b) Conductance curve $(\mathrm{G}(\mathrm{V}))$ obtained by numerical derivative of the $\mathrm{I}-\mathrm{V}$ in image $\mathrm{a})$.

The CITS image is composed of a regular array of pixels, in which an I(V) was acquired with the feedback turned off. At each pixel of the image, the system corrects the $\mathrm{Z}$ position of the tip to meet the setpoint conditions $(800 \mathrm{mV}, 200 \mathrm{pA})$. This correction motion is used to acquire a topography image at the same time that the spectroscopy is performed. This $\mathrm{Z}$ image is very useful for a fine correlation of the spectroscopic and real-space features. Figure 2.28 (a) shows roughly the same topography as in figure 2.26 , but with less resolution due to the decrease of the number of pixels of the image ${ }^{2}$.

Figure 2.28 (b) shows the ZBC map of the region. At each pixel, the numerical derivative of the $\mathrm{I}-\mathrm{V}$ curve is calculated and then evaluated at $V=0 V$, i.e., $\mathrm{G}(0)$ is the $\mathrm{ZBC}$. Homogeneous conduction along the area is obtained, with small jumps at the edge of the terrace. For a matter of clarity, figure 2.28 (c) shows the histogram of the ZBC map. These diagrams and the ZBC values will be the main tool used in

\footnotetext{
${ }^{2}$ Typically, STS experiments were done in a grid of 128 pixels x 128 pixels. At each pixel, 3-10 IV curves were averaged. Each I-V curve was acquired in $500 \mathrm{~ms}$, which means that one single STS map is acquired in 6-12 hours, depending on the conditions
} 

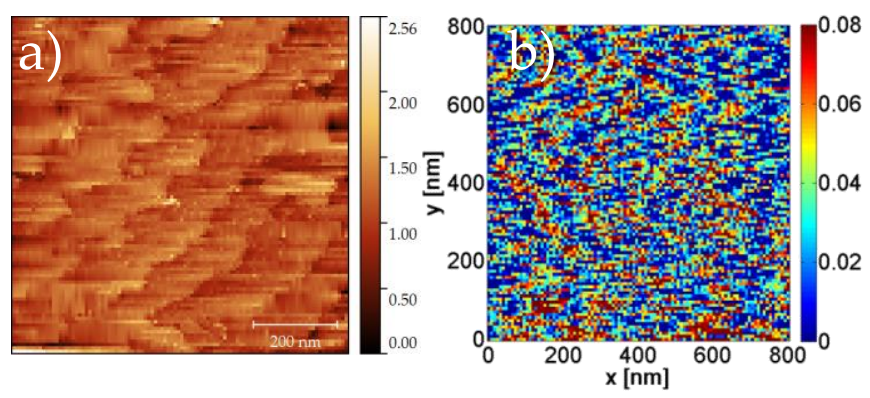

C)

FIGURE 2.28: a) Topography image obtained during the STS measurement. b) ZBC map of the region The map has been obtained by calculating the numerical derivative of the I-V curves acquired at each pixel of the image. Then, the $G(0)$ value is plotted for each pixel. c) Histogram of the ZBC of image b).

the discussion presented in chapter 4 in relation to the tunnel spectroscopic study of high resistances areas induced by RS effect.

\subsubsection{Atomic Force Microscopy}

The AFM was invented in 1986 by Binning, Quate and Gerber [194] and it was intended to overcome the limitations of STM, which can be only used on conductive samples. Its working principle is based on the measurement of the interaction forces $\left(10^{-13}-10^{-5} \mathrm{~N}\right)$ appearing between a nanometric tip and the specimen under investigation when they are very closed or even in contact. The tip is located at the end of a cantilever, which deflects when any force is applied to it. This deflection $\Delta \mathrm{x}$ is proportional to the $k$ constant of the (spring) cantilever according to Hooke's law, $\mathrm{F}=\Delta \mathrm{x} \cdot k$, and it is detected by an optical system. A laser beam reflects on the back part of the cantilever. The reflected beam hits on a photodetector divided in four sections (photodiodes) which allows to measure vertical and lateral deflection [203]. These deflections are registered during the scanning of the sample and are used to interpret the forces involved in the interaction. This force detection system was firstly introduced here [204,205] and nowadays it is the most common setup in all the commercial AFM. In this way, several local properties of the material such as the surface topography, friction, electrical resistivity or magnetization. A piezoelectric tube controls the $\mathrm{X}, \mathrm{Y}$ and $\mathrm{Z}$ position of the tip. Although atomic resolution can be achieved, typical lateral resolution ranges between 5 and $20 \mathrm{~nm}$, depending on the sharpness of the probe. Vertical distances can be measured with an accuracy as high as $\sim 0.1 \mathrm{~nm}$. Figure 2.29 shows a schematic of our AFM setup with all its components.

The AFM can also regulate the applied force through a feedback loop. This feedback loop acts to maintain any of the available variables constant, i.e., tip-sample distance, applied force, amplitude, phase or frequency, among others. A picture of the AFM system used in this Thesis is shown in part b of figure 2.29. It is a Keysight 5500LS, former Agilent 5500LS. Along this PhD Thesis, some modifications and extra capabilities were introduced in the system with the valuable help of Mr. Andres Gomez, technician of the SPM services at ICMAB. The modifications introduced in 
a)

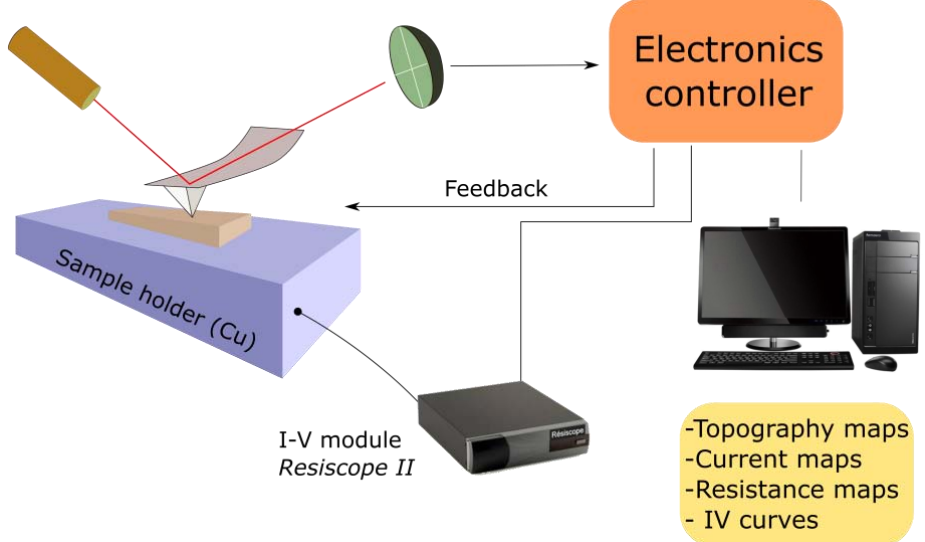

b)

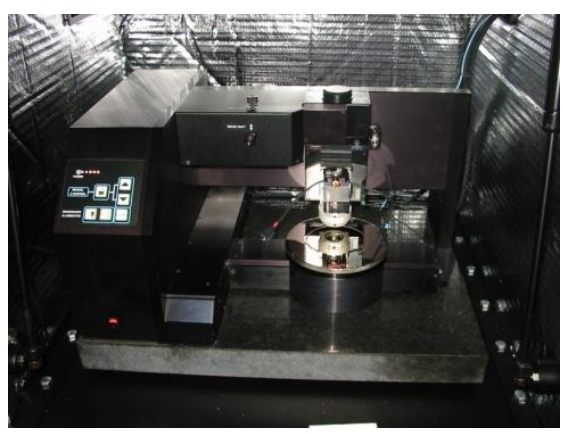

FIGURE 2.29: (a) Schematic representation of our AFM setup with all of the components, including the Current supplier called Resiscope II and will be introduced in next paragraph. (b) Real picture of the equipment employed during this thesis.

the system were experimental requirements of our measurements and will be detailed in next sections. For a deeper understanding of the technique, the reader is referred to the following references $[195,206]$.

\section{Operation modes}

Depending on the tip-sample distance, the forces governing the tip-sample interaction can vary its nature. The total force between tip and sample is composed of a combination of short and long range interactions:

1. Short range forces: These forces are repulsive and are detected at distances $\sim 1-3 \AA$. These repulsion comes from the overlapping of the electron clouds between cantilever and sample's atoms (Pauli principle).

2. Long-range forces: detected at distances $d>5 \AA$. They are mainly attractive. Few examples are capillary, Van der Walls, electrostatic and magnetic interactions.

Usually, these forces can be approximated with the Lennard-Jones potential.

$$
U(r)=4 U_{0}\left(\left(\frac{R_{a}}{r}\right)^{12}-\left(\frac{R_{a}}{r}\right)^{6}\right)
$$


where $U_{0}$ is the depth of the potential well, $\mathrm{r}$ is the distance between the atoms at the tip apex and the sample, and $\mathrm{R}_{a}$ is the distance at which $\mathrm{U}(\mathrm{r})$ is zero. The force derived from this potential $F=-\frac{\partial U(r)}{\partial r}$ is depicted in figure 2.30. From this diagram, different operation modes can be used, depending on the forces we are interested in: (short range (repulsive)) or long range (mainly attractive). Essentially, the AFM operation modes can be divided into the contact mode, which works in the repulsive regime and the dynamic mode, which works in the attractive regime and can be subdivided into non-contact and intermittent -contact modes.

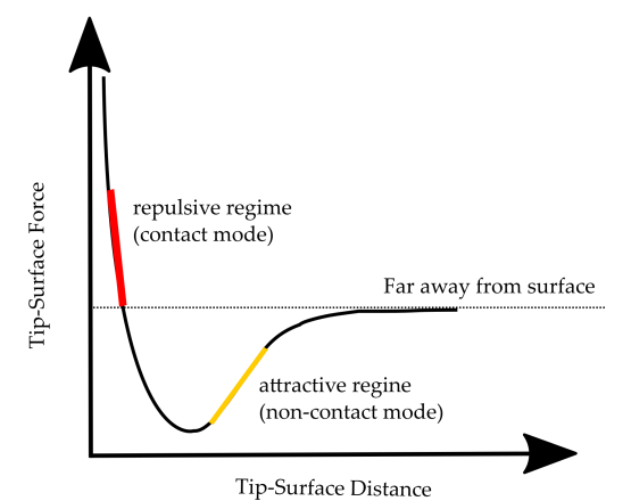

FIGURE 2.30: Interatomic forces dependency on the tip-sample distance and its working regimes.

The first developed mode was the Contact mode where tip and sample are in direct mechanical contact. By measuring the change in the vertical deflection at a constant applied force (load) as the tip scans over the surface, topographic images are obtained. Simultaneously, other physical quantities can be obtained, such as friction maps or conductivity (resistance) maps. This latter technique is called ConductiveAFM (C-AFM), and a more detailed explanation is offered in next section. In the dynamic modes the cantilever is oscillating near its resonance frequency at a given distance from the sample surface. Depending on the oscillation amplitude when compared to the tip-sample distance, the tip may either not touch the sample on every oscillation (non-contact mode, small amplitude) or touch it intermittently (larger amplitude). In this Thesis, the main operation mode employed has been the contact-mode for electrical characterization (C-AFM), and the setup is properly described below. For a more detailed review on the dynamics mode, the reader is referred to the following references[195, 206, 207].

AFM is not only a very versatile technique for surface characterization, but it can also serve as an active mechanism for material modification[197, 208]. Challenges in lithography have triggered a lot of interest in the research community due to its capabilities to manipulate materials properties at the nanoscale and even at the atomic level. There are a large variety of phenomena that can occur when a nanometric sharp tip is in contact with a surface. These phenomena include mechanical, thermal, electrostatic or chemical interactions, and/or a combination of them [197]. To mention some examples, one can oxidize $\mathrm{Si}$ to $\mathrm{SiO}_{2}$ to generate nanometer patterns [209], switch between different ferroelectric polarization states [210-212], induce a resistance change by applying large pressures with the AFM tip inducing a phase transition[213, 214]. 


\section{Conductive-Atomic Force Microscope}

As it was previously introduced, C-AFM technique [63] allows the simultaneous acquisition of both topography and current (resistance) maps, enabling to correlate spatial features of the surface to a certain conductivity state. Generally speaking, these current (resistance) maps are acquire by applying a constant bias voltage to the sample while the tip scans over the selected area. C-AFM can also perform I-V curves at a fix position. By freezing $X-Y$ position, a voltage ramp is applied to the sample and the flowing current is recorded. In following sections it will be shown that this tool will be key to understand our RS mechanism.

The first requirement to perform electrical measurements by C-AFM is to have a conductive tip. The most typical C-AFM tips commercially available are made out of $\mathrm{Si}$ with a coating on both sides of the tip of $\mathrm{Cr} / \mathrm{Pt}$. In our case, these kind of tips were damaged quite soon due to the big loads applied. To reduce the wear of the tip, in all the experiments shown in this thesis, unless it states the contrary, have been performed with a diamond coated Bruker DDESP V2 tips. A picture of the tip and some information provided by the supplier can be found in figure 2.31. The nominal $k$ value of these tips is $80 \mathrm{~N} / \mathrm{m}$. This high $k$ values exerts large forces (nN $-\mu \mathrm{N}$ regime). In our case, we observed that even though the high loads applied, no damage was induced to the sample plus a better electric contact between tip and sample was achieved.

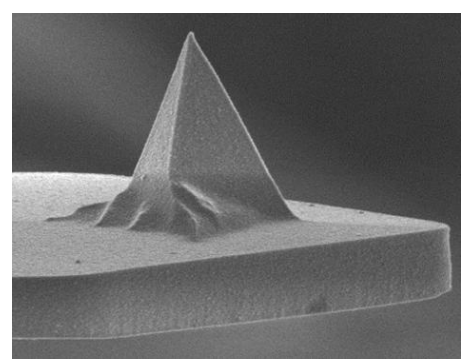

$\begin{array}{ll}\text { Geometry: } & \text { Standard (Steep) } \\ \text { Tip Height (h): } & 10-15 \mu \mathrm{m} \\ \text { Tip Radius (Nom): } & 100 \mathrm{~nm} \\ \text { Tip Radius (Max): } & 150 \mathrm{~nm} \\ \text { Tip Coating: } & \text { Conductive Diamond } \\ \text { Nominal } k: & 80 \mathrm{~N} / \mathrm{m}\end{array}$

FIGURE 2.31: SEM image of the AFM tips used in this Thesis and information provided by the supplier. Both were taken from the supplier's webpage. Its nominal spring constant $k=80 \mathrm{~N} / \mathrm{m}$.

The second requirement to perform these measurements is to have a good preamplifier to enhance the current limits that commercially available AFMs have. In our case, the current range of the equipment is $0.1 \mathrm{nA}-100 \mathrm{nA}$. For our experimental purposes, a broader current range was desired. In this sense, the Resiscope II module of CSInstruments (figure 2.29) was acquired and installed for our C-AFM experiments. Resiscope extends the accessible current range from $100 \mathrm{fA}$ to $1 \mathrm{~mA}$. This system allows to measure over 10 decades with high sensitivity and resolution by applying a $V_{\text {bias }}$ to the sample. Both mapping and I-V spectroscopy are compatible when using Resiscope module. Due to the nanometric size of the tip, large electric fields are created below the tip apex. Depending on the material and the atmosphere, several phenomena such as local oxidation $[208,209,215]$ might take place due to the presence of a water meniscus around the tip. 


\section{Protocol for RS measurement by C-AFM}

In this section, our experimental setup to perform RS studies is detailed. Some examples are shown in order to illustrate basic phenomenology without entering into any detail regarding the physical mechanism responsible of these results. Results and discussion will be further explained in next chapters.

Thanks to the intrinsic metallic character of the materials studied in this thesis (LSMO, YBCO and RENO), we can intentionally avoid the usual MIM structures used to study RS. In our case, as it was mentioned before, the substrates are insulating (STO,LAO) and the grown thin film act both as an active element for RS and as electrode material. Figure 2.32 shows a diagram of the electrical connections for C-AFM measurement. The sample is glued to the sample holder, which is a solid piece of copper. Voltage is applied to the sample holder, while the conductive tip remains grounded. The connection between the copper holder and the sample surface is made with a silver wire (from Goodfellow Inc.) attached with conductive silver paste (from RS Components).

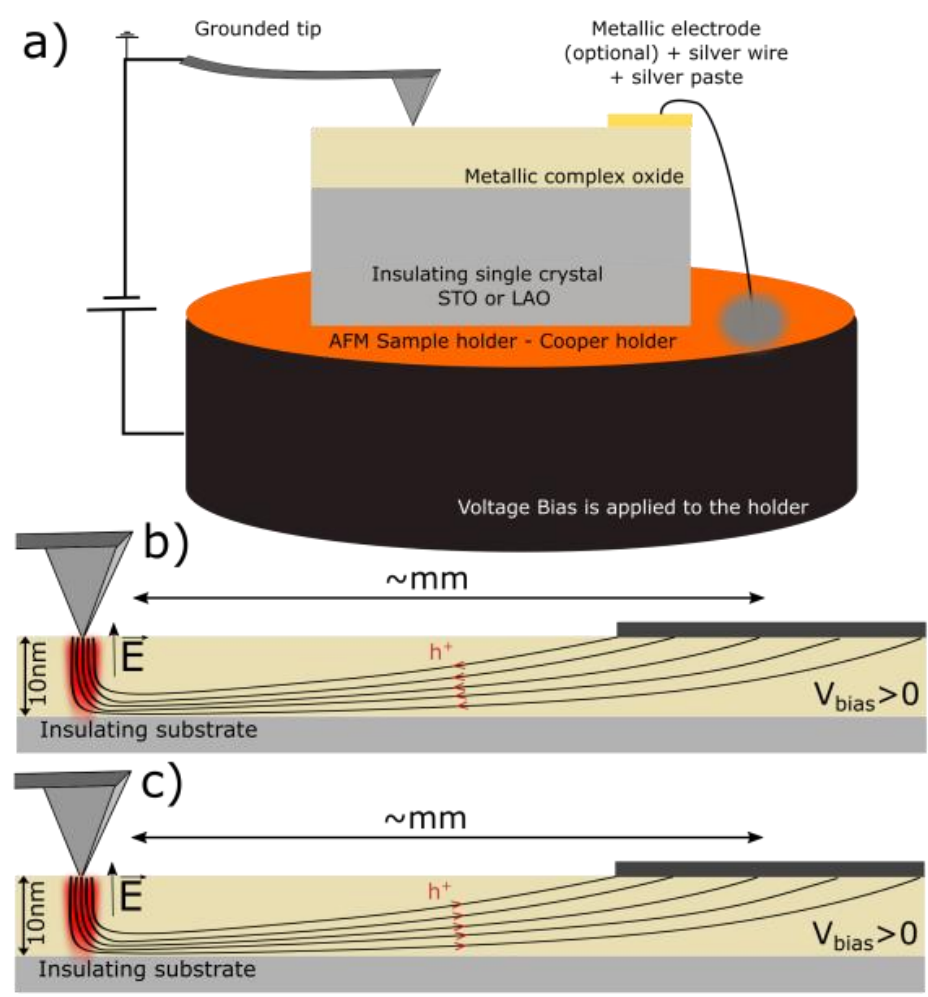

FIGURE 2.32: (a) Schematic representation of the AFM setup, showing the wire connection between the $\mathrm{Cu}$ sample holder and the surface of the metallic perovskite. The sample is glued to the holder. Tip is grounded and the sample is biased. (b) and (c) shows a diagram of the electric field distribution when positive and negative bias is applied, respectively.

Figure 2.32 (b) and (c) also show schematically how the current flow from the tip to the electrode under a positive bias (b) and viceversa under negative bias (c) (tip). Although the metallic character of the films, the contact between tip and sample is not a perfect ohmnic contact and therefore there is a large contact resistance in between. This produces that the voltage drops mostly below the tip in a vertical 
way, and then the electric field spreads within the film towards the electrode. This diagram is crucial to understand the switching behaviour in these materials since we realize that the material transformation occurs mainly below the tip, in a vertical manner.

As it will be shown in chapters 3 and 4, we do not observe any feature that could suggest a strong bending of the electric field close to the tip. Instead, the modifications that will be later induced in the films present well-defined features which suggest, in first approximation, that the high concentration of electric field lines below the tip leads to the domination of the vertical component rather than the lateral one (probably the lateral component might be in the $\mathrm{mm}$ range at rather lower electric field values to close the circuit). Therefore, we do not have any evidence of an electric field bending dominating our switching effect. On the contrary, at the local scale (up to $\sim 10 \mathrm{~nm}$ ), we demonstrate that the electric field is mainly directed upwards and downwards.

For the sake of completeness, figure 2.33 shows a typical topography (a) obtained simultaneously while acquiring the current (resistance) values at $1 \mathrm{~V}(\mathrm{~b})$ for a typical $6 \mathrm{~nm}$ LSMO film. In both topography and resistance maps the step-like terraces can be clearly observed. Also a typical I-V curve obtained in a certain point at the surface at low bias is shown, where a quasi-ohmic behaviour is observed.

a)

b)

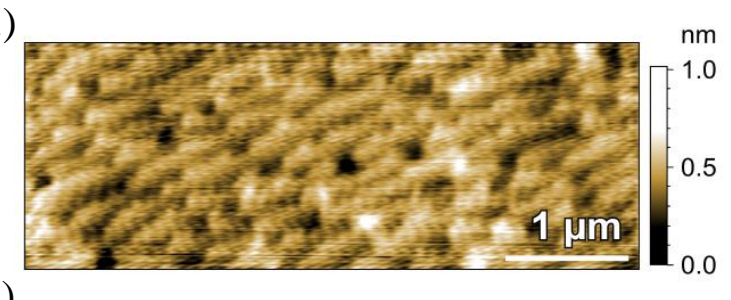

c)

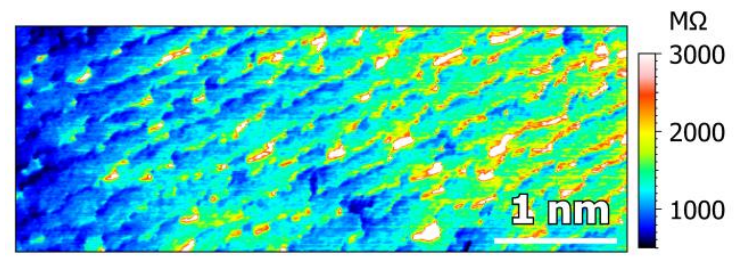

FIGURE 2.33: (a) Topography obtained in a $6 \mathrm{~nm}$ LSMO. (b) Resistance map acquired at $1 \mathrm{~V}$ simultaneously. Step-like terraces are observed both in topography and in resistance. (c) Typical I-V curve performed at low bias in an LSMO sample on top-top configuration.

Regarding the applied load on the tip, it is important to minimize the contact resistance between tip and sample to assure a good electrical contact. One can naively think that to do so it is enough to apply as much force as possible. However, some modifications in the film and extra effect might be induced by the tip when applying high loads $[213,214]$. We acquired Current-Force curves (I-F) in our films at a fix voltage $(V=1 V)$ as shown in figure 2.34. The experiments are performed by retracting the tip far from the surface and starting the approach to the sample while recording the current as a function of the force.

The graph shows that even at higher loads no substantial change in the current is observed and a plateau is reached. This means that the current does not depend too much on the applied load at intermediate and high loads. In conclusion, for all 
FIGURE 2.34: I-F curves with a DDESP-V2 tip at 1V.

of our experiments, the applied load will not play a role in the interpretation of the experiments and our working regime will be from 1-5 $\mu \mathrm{N}$.

The presence of high humidity levels enhances the present of the water meniscus surrounding the AFM tip $[209,216]$. The influence of the water meniscus in regular topography measurements and specially, when high bias is applied, can be crucial and have a strong impact on the film. It has been mentioned before the amount of effects that can be induced by the AFM tip. These effects can have a huge impact when the water meniscus is enhanced. To determine the influence of ambient humidity (and therefore, the water meniscus) in our bias-induced effect, two different experiments conducted at ambient and low humidity atmospheres, on YBCO thin films, are performed. We have chosen to show an example with YBCO due to its extreme sensitivity to humidity and water in its surface [217-219]. The first experiment is shown in figure 2.35. It was performed at ambient relative humidity (RH) $\sim 55 \%$. First, the scan area is set to $10 \times 10 \mu m^{2}$ with $V_{\text {Bias }}=1 \mathrm{~V}$ to characterize the topography and the resistance of the pristine area. After that, small squares of $1 \times 1 \mu \mathrm{m}^{2}$ were scanned at high negative bias (with the values shown in the figure) to induce a resistive switching effect. Then, the scan area is set again to $10 \times 10 \mu \mathrm{m}^{2}$ and $1 \mathrm{~V}$. The resistance map obtained at $1 \mathrm{~V}$ shows larger areas than the $\mu m^{2}$ squares scanned are transformed. This resistance change is accompanied by a topography modification.

The topography and resistance change induced in these areas are not reversible, i.e., if the reverse polarity would be applied, only more damage would be infringe to the film. In contrast, if the same experiment is performed under a low $\mathrm{RH}(<$ $5-10 \%$ ) as in figure 2.36 , the results are very different.

Figure 2.36 (a) and (b) show the $\mathrm{R}$ maps for the pristine state and for the HRS induced at $-6 \mathrm{~V}$ in a $2 \times 2 \mu \mathrm{m}^{2}$. The HR square now does not extends several $\mu$ as in the first example, but it is rather limited to the set writing area. Image $c$ ) and d) show the average profile for both topography and resistance maps respectively. These profiles are obtained by averaging all the scan lines (typically 512 profiles are averaged). While the resistance has increased by 2 orders of magnitude due to the RS effect, the topography remains unaffected, and therefore reversibility can be achieved, as it is shown in the YBCO section in chapter 4 .

At the beginning of the PhD thesis, the used equipment (Agilent 5500LS) was not able to measure at low humidity conditions. To set up the system, two small holes 

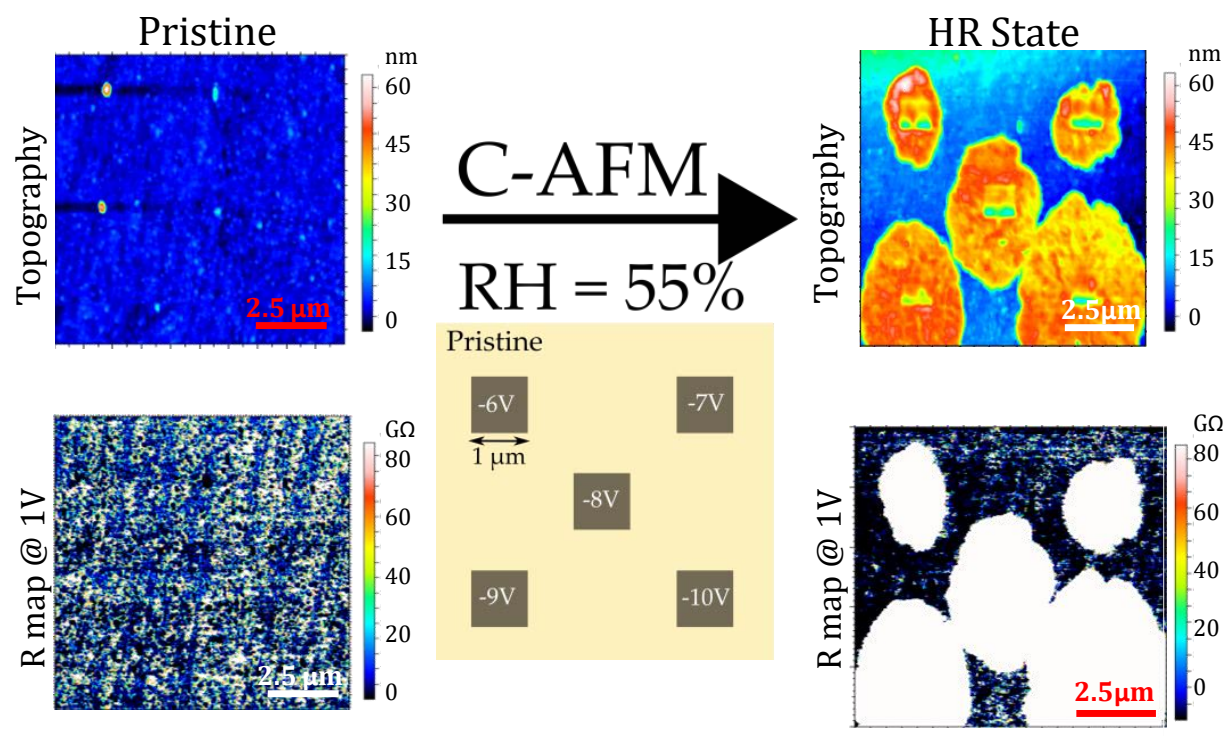

FIGURE 2.35: Topography and resistance map of the selected area before and after nanostructuration. The experiment was performed at $R H=55 \%$.

were drilled in the external chamber to facilitate the entrance of dry air and nitrogen pipes. Dry air allows to reach even 5-6\% relative humidity, while with nitrogen $R H \sim 2 \%$ can be reached. Since the AFM chamber was not sealed, a flow of these gases was maintained during the experiments to keep the low humidity atmosphere conditions, making sure that no distortion was occurring to the tip.

\subsubsection{Probe Station measurements for $\mathrm{RS}$ at a micrometer scale}

Probe Station measurements using two- and four-probe configuration (this latter, for 3-terminal devices) were used with Semiconductor Parameter Analyzers to induce and evaluate the resistive switching behaviour in the different materials. The measurements were performed at the Department of Electrical Engineering (UAB) and at Peter Grüngberg Institut at the Forschungszentrum Jülich (FZJ).

At $U A B$, the experiments were carried out in collaboration with Dr. Rafael OrtegaHernandez (former member of the group) and Prof. Jordi Suñe. The measurements were performed using either the Keithley 4200-Semiconductor Characterization System and the Agilent 4156C Semiconductor Parameter Analyzer (2.37 (a)). In all of our measurements, the sample was placed inside of a probe station which is insulated as a Faraday shield in order to avoid electric interferences. In addition, the sample was firmly attached to the sample holder through a vacuum system and the micrometric probes were placed manually on the sample. I-V measurements were conducted through these two probes which have been configured by software to apply voltage ramps while sensing the current (2.37 (b)).

At FJZ, the experiments were performed during a two weeks short stay in the group of Prof. Regina Dittmann and experiments were carried out in collaboration with Mr. Benedikt Arndt. The analysis of the data was performed by me. 


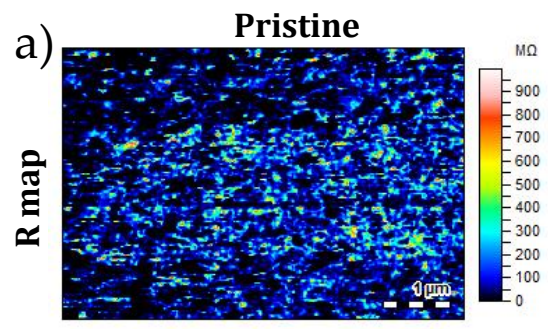

c)

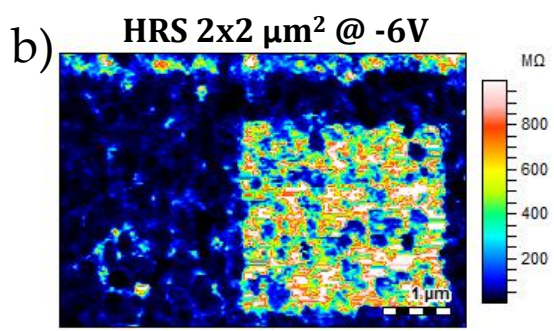

d)

FIGURE 2.36: Micrometric HR states in YBCO thin film. (a) R map of the pristine state acquired at $1 \mathrm{~V}$. (b) $\mathrm{R}$ map at $1 \mathrm{~V}$ after inducing a $2 \times 2 \mu m^{2}$ square at $-6 \mathrm{~V}$. (c) Average topographic profiles in the same area as Rmap in image $a d$ ) Average resistance profile for $b$.

a)

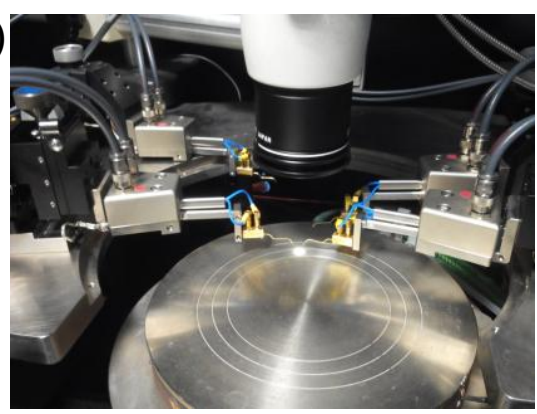

b)

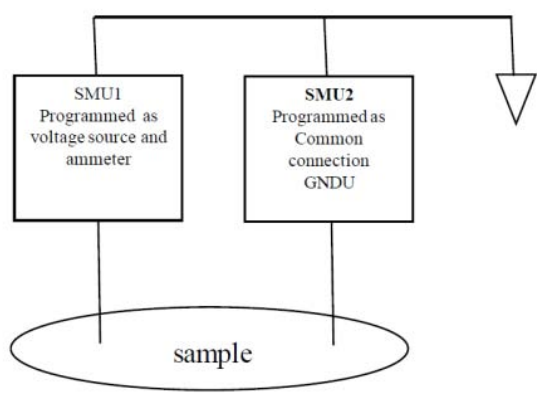

FIGURE 2.37: (a) Setup employed at the UAB facilities. (b) Diagram of the measurement procedure in probe station systems.

The experimental setup consisted of a two-probe home-made configuration system with similar functionalities as the one described above. The tips were placed inside a sealed chamber with different gas inlets and connected to a rotatory pump. Vacuum conditions down to $P=10^{-5}$ mbar can be achieved in this system. Besides, gas inlets allow to perform measurements at different gas partial pressures. In particular, pure $\mathrm{O}_{2}$ atmosphere at different pressures was also used in the experiments. Figure 2.38 shows a picture of the actual equipment and a schematic diagram of it. The same electric field configuration than in our AFM setup was used.

\subsubsection{Other characterization techniques}

The aim of this section is to state the different contributions and collaborations established to allow the use of other characterization techniques during the development of this thesis. Further information about the working principles of the techniques mentioned below is provided in Appendix A. 


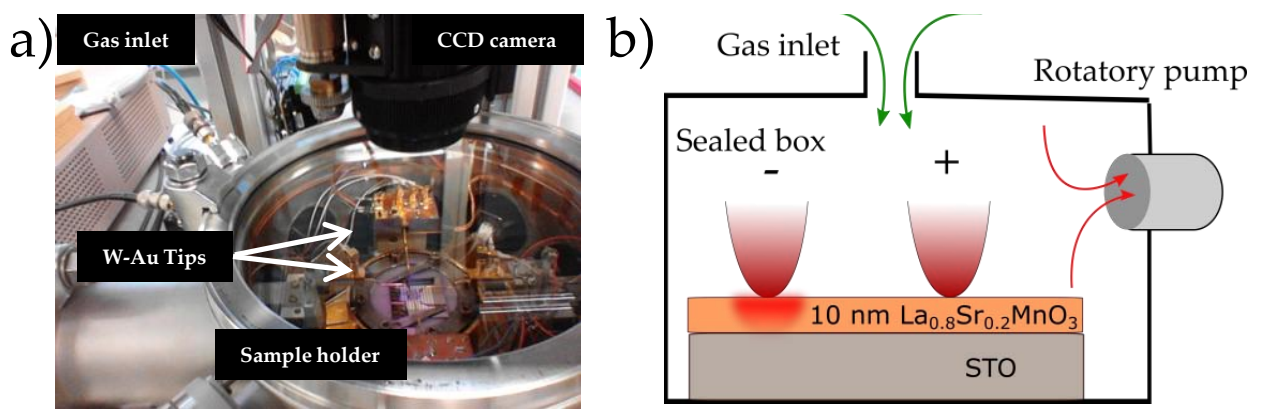

FIGURE 2.38: (a) Real picture of the equipment and its main components used in FJZ. (b) Schematic diagram of the experiment. Negative tip will be the active RS element.

Besides the STM and AFM characterization mentioned above, Scanning Electron Microscopy was used to reveal the morphological features of the film. Measurements of samples and subsequent analysis of data were conducted by me. For the structural characterization of the films, we have used X-ray diffraction (XRD) and Transmission Electron Microscopy (TEM) techniques. For XRD, most of the measurements conducted at ICMAB were performed by the technicians (A. Crespi, F. J. Campos and J.Esquius). All data obtained was completely analyzed by me. The TEM samples preparation and measurements were conducted by Mr. Bernat Mundet as a part of his PhD and supervised by Dr. Jaume Gazquez, both members of our research group. Measurements were carried out at the Advanced Microscopy Laboratory from the Institute of Nanoscience of Aragon (LMA-INA), in Zaragoza, and at the TEM facilities of the Institut Catala de Nanociencia i Nanotecnologia (ICN2). Resistivity and transport properties measurements were conducted in Physical Properties Measurement System (PPMS) from Quantum Design. The experiments and data anaylisis were carried out by me. Induction measurements were performed in a Superconducting Quantum Interference Device (SQUID) at the same laboratory. Measurements were mainly performed by the technician (Dr. Bernat Bozzo) while data analysis was fully done by me.

\subsection{Conclusions}

In this chapter, the growth process of $\mathrm{La}_{1-x} \mathrm{Sr}_{x} \mathrm{MnO}_{3}, \mathrm{RENiO}_{3}, \mathrm{YBa}_{2} \mathrm{Cu}_{3} \mathrm{O}_{7-\delta}$ and $\mathrm{CeO}_{2-x}$ thin films have been presented. Chemical Solution Deposition method has been demonstrated to be a suitable route to obtain high-quality complex oxides thin films (LSMO and RENO), while Pulsed Laser Deposition has been employed to obtain YBCO thin films. Epitaxial films with smooth surfaces and low roughness $(0.6 \mathrm{~nm}<R M S<2 \mathrm{~nm})$ are obtained for the three compounds.

In the $\mathrm{La}_{1-x} \mathrm{Sr}_{x} \mathrm{MnO}_{3}$ case, the films were grown onto STO substrates with thickness ranging from 3 to $42 \mathrm{~nm}$. The doping level $x$ have been varied by modifying the $\mathrm{Sr}$ content of the films. In this way, the metal-insulator transition and the resistivity of the material has been tuned. In the $\mathrm{RENiO}_{3}$ case, $\mathrm{LaNiO}_{3}$ and $\mathrm{NdNiO}_{3}$ films were grown onto LAO and STO substrates with thickness ranging from 6 to $25 \mathrm{~nm}$. LNO films does not present MIT in the full temperature range for none of the thickness studied. In contrast, NNO thin films grown on LAO present a 
thickness-dependent MIT. In the YBCO case, the films were grown on STO substrates with thickness ranging from 10 to $200 \mathrm{~nm}$. High quality samples with excellent superconducting performance are obtained. For films with thickness below $50 \mathrm{~nm}$, degradation of the superconducting properties, such as the critical temperature $T_{c}$ and the critical current density $J_{c}$, are observed. In addition, 3-10 $\mathrm{nm} \mathrm{CeO}_{2}$ thin layers were ex-situ deposited by Atomic Layer Deposition on top of LSMO and YBCO thin films. The physical properties of the underlying metallic perovskite remains unaffected after the deposition process.

The main tools to induce and characterize the resistive switching behaviour of the films have been introduced: Atomic Force Microscopy (AFM), Scanning Tunnelling Microscopy (STM) and probe station measurements. In the case of the STM, special emphasis has been given in the relation between the number of available states at the Fermi level and the zero bias conductance (ZBC), which is an observable upon tunnelling spectroscopy. In the case of the AFM, we have introduced the Conducting-AFM technique, which will be the main tool used to induce the resistive switching effect in our films. Special attention has been put to the decrease of the relative humidity of the air inside the chamber. This precaution avoids irreversible damage of the film due to the presence of the water meniscus upon application of a very large electric field generated at the tip apex.

Finally, we stated the different characterization techniques employed in the development of the thesis, and my contribution. 



\section{Chapter 3}

\section{Resistive Switching in LSMO thin films}

In this chapter, we will present our results regarding the characterization of the resistive switching phenomenon in $\mathrm{La}_{1-x} \mathrm{Sr}_{x} \mathrm{MnO}_{3}$ compounds. Strong evidences supporting a MIT-electric-field-induced transition with oxygen exchange through ambient will be provided.

After the first observation of a resistance change effect in $\operatorname{Pr}_{0.7} \mathrm{Ca}_{0.3} \mathrm{MnO}_{3}$ (PCMO) single crystals [46], there was a renewed interest in perovskite manganite materials with the observation of a resistance change induced by electrical pulses in $\operatorname{Pr}_{0.7} \mathrm{Ca}_{0.3} \mathrm{MnO}_{3}$ thin films [220]. Several works addressed the RS capabilities in PCMO [21, 22, 221-225], $\mathrm{La}_{1-x} \mathrm{Co}_{x} \mathrm{MnO}_{3}$ [226-228] and $\mathrm{La}_{1-x} \mathrm{Sr}_{x} \mathrm{MnO}_{3}$ [50, 229233]. Depending on the material and device structure, multiple microscopic mechanisms have been proposed based either on redox processes involving cation or anion migration [221], on the formation/rupture of conducting nanoscale filaments [17, 139, 234-236], oxygen diffusion [224, 237], Mott metal-insulator transition and strong-correlated effects [21, 22, 32, 79], variable Schottky barrier [30, 222], or charge trapping at interfacial sites [223]. In prior work from the group [50, 51], a volume switching effect of $\mathrm{La}_{0.7} \mathrm{Sr}_{0.3} \mathrm{MnO}_{3}$ was suggested as a consequence of a metal-insulator transition. A very recent report from L. Yao et al [238] has suggested an oxygen vacancy-driven structural and resistive phase transitions in $\mathrm{La}_{2 / 3} \mathrm{Sr}_{1 / 3} \mathrm{MnO}_{3}$. In this thesis, we have thoroughly studied the RS effect exhibited by different techniques and extended the studies to metallic perovskite oxides (including $\mathrm{La}_{1-x} \mathrm{Sr}_{x} \mathrm{MnO}_{3}, \mathrm{YBa}_{2} \mathrm{Cu}_{3} \mathrm{O}_{7-\delta}$ and $\mathrm{RENiO}_{3}$ ). We have elaborated a description of the effect that may be of of general validity for other metallic perovskite oxides with strong electron interactions and displaying MIT, thus, making these systems very appealing candidates for non-volatile memories and other novel devices.

It is worth to mention that parallel to this thesis, another doctoral work [156] has been developed in a similar topic. In his thesis, Dr. Rafael Ortega-Hernandez has studied the RS-MIT properties of $\mathrm{La}_{0.8} \mathrm{Sr}_{0.2} \mathrm{MnO}_{3}$ and $\mathrm{CeO}_{2} / \mathrm{La}_{0.8} \mathrm{Sr}_{0.2} \mathrm{MnO}_{3}$ thin films devices with $\mathrm{W}$-Au macroscopic tips. The measurements were performed in a semiconductor parameter analyser, fruit of a collaboration with the School of Engineering of the Universitat Autònoma de Barcelona with Prof. Jordi Suñé. Along this chapter, special emphasis will be made where mutual collaboration has been beneficial. 


\subsection{Resistive Switching in LSMO films studied by C-AFM}

In this section, we present our studies on the RS behaviour of LSMO thin films using C-AFM. Local point-contact electric measurements (I-V sweeps) and micrometric arrays, generated at different bias voltage, are performed in the bare surface of LSMO films to reveal its intrinsic RS properties.

Local probe techniques are widely used in RS studies. Several works address proofof-principle memory element miniaturization because of the nanometric resolution achieved by C-AFM [239-241]. C-AFM also succeeded in the observation of conducting filaments in insulating matrices, such as $\mathrm{TiO}_{2}[188,234,242], \mathrm{SrTiO}_{3}$ [24, 139] and $\mathrm{NiO}[18,235,236,243]$, and in combination with other characterization techniques such as TEM [17,19] or TOF-SIMS [244-246]. A more detailed review is provided in reference [247].

\subsubsection{Electrical characterization by I-V sweeps}

\section{Saturated I-V sweeps}

It is worth reminding that the measurements were performed under low humidity atmosphere $(<5-10 \%)$ kept under constant flow of dry air and/or $\mathrm{N}_{2}$ gas. This highly increases reproducibility and avoids deterioration of the sample by the water meniscus under the tip.

A typical I-V curve observed in a $35 \mathrm{~nm} \mathrm{La}{ }_{0.7} \mathrm{Sr}_{0.3} \mathrm{MnO}_{3}$ thin film is shown in figure 3.1. The applied voltage sweep is composed of several branches and are described as follows:

1. $0 \longrightarrow-7 \mathrm{~V}$ : The first sweep starts towards negative bias. It is in this branch where the change from the initial pristine, which is the Low Resistance State (LRS), to a High Resistance State (HRS) is induced. The generated electric field points downwards and favours oxygen excorporation from the crystal structure (generating oxygen vacancies) together with the corresponding valence change in the metal Mn cations $\left(\mathrm{Mn}^{4+} \rightarrow \mathrm{Mn}^{3+}\right)$ concomitant with a decrease in the number of carriers (holes) in the system. This induces an increase of resistance of the material and eventually the MIT can be crossed, as it will be shown in sections 3.2 and 3.3.

2. $-7 \longrightarrow 0 \mathrm{~V}:$ In this branch, the system remains in the HRS when the sweep goes back to $0 \mathrm{~V}$.

3. $0 \longrightarrow 7 \mathrm{~V}$ : The sweep starts in the HRS and a transition from HRS to the LRS emerges in this branch at a given threshold voltage. At this transition, the electric field points upwards and induces oxygen incorporation into the structure and the recombination with oxygen vacancies. Concomitant to it, the reverse valence change in the Mn cations $\left(M^{3+} \rightarrow M n^{4+}\right)$ and an increase in the carrier density occur. This decreases the resistance of the system and the MIT can be crossed back.

4. $7 \longrightarrow 0 \mathrm{~V}$ : In this region, the system remains in the LRS, finishing the voltage excursion at $0 \mathrm{~V}$. Then, the cycle is repeated to show its reproducibility.

A clear asymmetry in the I-V sweep is observed. The transition from the LRS to the HRS is performed smoothly while the switching from the HRS to the LRS presents a 


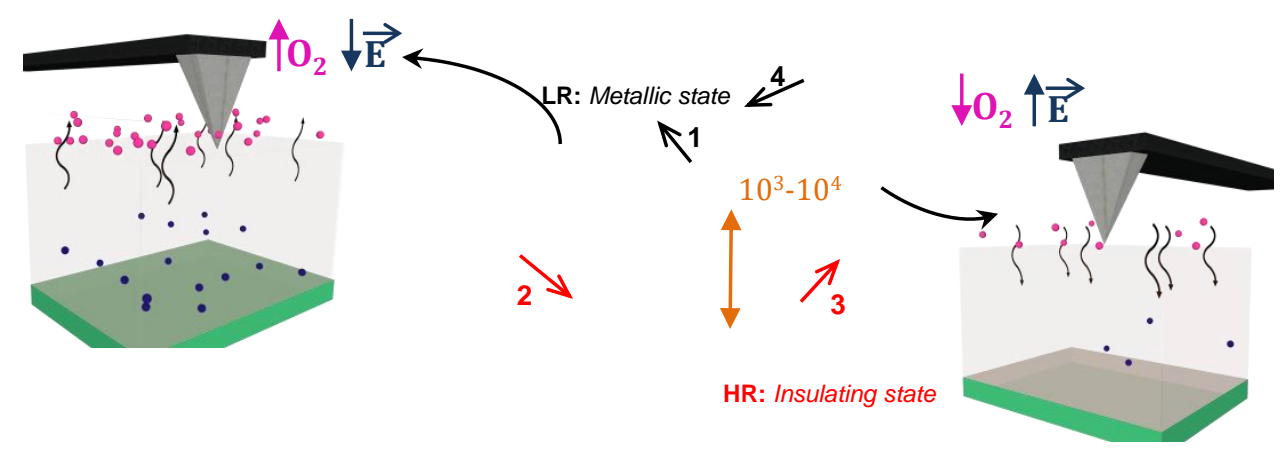

FIGURE 3.1: Typical IV curve in LSMO thin film. Insets show the excorporation (left) and incorporation (right) processes.

sharp transition. We believe that this asymmetry is influenced by two factors. First, there is an inherent geometrical asymmetry since the nanometric tip acts as an electrode whereas the counterelectrode is at least 5 orders of magnitude larger. Second, oxygen incorporation and excorporation processes are intrinsically different when the exchange is produced with the atmosphere (oxygen molecule dissociation or association) and therefore, a symmetric sweep is not expected. When oxygen exchange with ambient is avoided by creating a bilayer system $\left(\mathrm{CeO}_{2} / \mathrm{LSMO}\right)$ with symmetric electrodes, in which the $\mathrm{O}^{2-}$ is only exchanged through the bilayer, the I-V sweeps present more symmetric sweeps with sharp and abrupt transitions in both polarities [248], as it will be shown later. Threshold voltages $V_{\text {HRS } \rightarrow \text { LRS }}$ range between $3-5 V$ (more detailed experiments on this regard are presented afterwards). In the ON state (LRS), the current values reach the $0.1-10 \mu A$ whereas in the OFF state (HRS) it is found to be in the $0.1-10 n A$ range. Resistances ratios of $10^{3}-10^{4}$ are thus obtained. We believe that such large resistance ratios are induced with a small change in the carrier density due to the strong electron correlation of the LSMO films governed with a MIT [249].

The observed RS physical mechanism is inherently bipolar, i.e., one can only switch the state under the proper bias polarization. To demonstrate that, we undertook unidirectional I-V sweeps on a $10 \mathrm{~nm}$ LSMO film only towards positive bias $(0 \mathrm{~V} \rightarrow$ $\left.+V_{\max } \rightarrow 0 \mathrm{~V}\right)$ (figure 3.2 (a) and later only towards negative bias $\left(0 \mathrm{~V} \rightarrow-V_{\max } \rightarrow\right.$ $0 V$ ) (figure $3.2(\mathrm{~b})$ by progressively increasing the amplitude of $\mathrm{V}_{\max }$. For each polarity the sweeps were acquired on a pristine spot of the surface. For positive bias sweeping, no switching hysteresis occurs. The resistance state of the sample remains in a LRS, which is in the initial pristine state, since no more oxygen can enter into the structure.

In contrast, a progressive hysteretic behaviour is found for the negative bias, since the electric field created in such a measurement reduces the concentration of hole carriers and moves $O^{2-}$ species from the crystal towards the surface. At the surface, these ions recombine with adsorbates forming $\mathrm{O}_{2}$ molecules, which are liberated. In this way, an oxygen vacancy is created into the system, with the corresponding change in the valence of the $3 \mathrm{~d}$ metal cations. The continuous increase of the HRS with increasing $-V_{\max }$ shows the capabilities of these systems to achieve multilevel resistive states. The basic phenomenology of the RS behaviour by means of I-V characteristics in LSMO thin films is thus presented. 
a)

b)

FIGURE 3.2: Unidirectional I-V curves obtained by C-AFM in a 10 nm LSMO film. (a) Towards positive bias. (b) Towards negative bias.

\section{Minor loops and initial state}

The example of a typical I-V shown in figure 3.1 shows a fully developed HRS and a complete transition back to the LRS. In the next experiment, we evaluate the I-V sweeps of a $35 \mathrm{~nm} \mathrm{La}{ }_{0.7} \mathrm{Sr}_{0.3} \mathrm{MnO}_{3}$ thin film when the maximum voltage amplitude of the sweeps is progressively increased. Each I-V curve was performed on pristine regions following the protocol defined as $0 \mathrm{~V} \longrightarrow-\mathrm{XV} \longrightarrow+X V \longrightarrow 0 \mathrm{~V}$ with $|X|=\{2,3,4 \ldots 8\}$, as shown in figure 3.3.

FIGURE 3.3: I-V sweeps performed in a $35 \mathrm{~nm}_{0.7} \mathrm{Sr}_{0.2} \mathrm{MnO}_{3}$ sample.

Each colour represents a a different maximum voltage amplitude.

All sweeps start towards negative bias and back to $0 \mathrm{~V}$ (branches 1 and 2). They start in a LRS as expected from the metallic state of the $\mathrm{La}_{0.7} \mathrm{Sr}_{0.3} \mathrm{MnO}_{3}$ films at room temperature. A continuous broadening of the RS hysteresis cycle is observed as the length of the sweep is increased. Therefore, the generated HRS can be finely tuned with the maximum applied voltage of the sweep. Then, we induce sweeps towards positive bias with same voltage amplitude but with reverse polarity than in the negative branch. In every case, an $\mathrm{OFF} \rightarrow \mathrm{ON}$ transition is observed. The threshold voltage at which this transition, $\mathrm{V}_{\mathrm{HRS} \rightarrow \mathrm{LRS}}$, occurs, correlates with the previously applied negative sweep. The larger the amplitude applied in the negative sweep, the larger threshold voltage. A similar correlation is inferred in the resistance ratio $R_{\mathrm{OFF}} / R_{\mathrm{ON}}$. A larger ratio is also found in the positive branch in the sweeps where large negative bias was applied. A saturation value of the ratio and the threshold voltage is reached for sweeps with $\left|V_{\max }\right| \sim 6 \mathrm{~V}$. For sweeps 
with smaller amplitudes, a minor loop situation is generated. This demonstrates the multilevel switching capabilities of LSMO films.

The RS hysteresis cycle shown in figure 3.1 illustrates how an ideal saturated RS cycle looks like in a LSMO thin film. However, different behaviours can be found when sweeping on the bare surface of the film. Two of these cases are shown in figure 3.4.
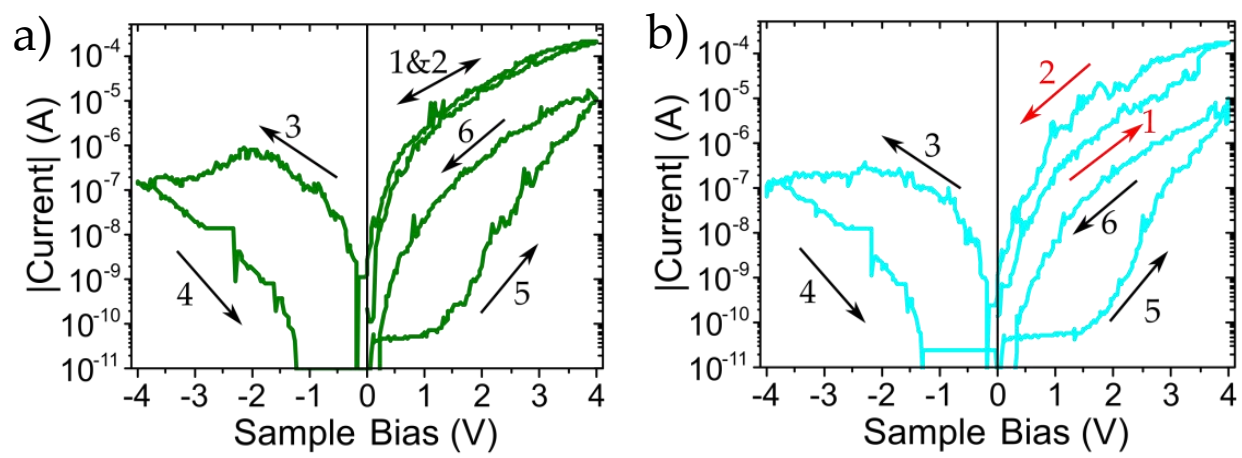

FIGURE 3.4: I-V sweeps starting towards positive bias. In (a), a fully reversible initial sweep is found, whereas in (b) a small transition to a lower LRS is observed.

In both examples the same sweep was applied, i.e., $0 \mathrm{~V} \rightarrow+4 \mathrm{~V} \rightarrow 0 \mathrm{~V} \rightarrow-4 \mathrm{~V} \rightarrow$ $0 \mathrm{~V} \rightarrow+4 \mathrm{~V} \rightarrow 0 \mathrm{~V}$. Since the initial state of the film is its pristine LRS, no change in the resistance is expected when a $V_{\text {bias }}>0$ is firstly applied, since it would induce oxygen incorporation. Only reversible metallic behaviour should be measured in this case by assuming a perfect oxygen stoichiometry in the region below the tip. This is the case showed in figure 3.4 (a). No trace of hysteresis or transition is observed in branches 1 and 2. However, in the example shown in figure 3.4 (b) a small abrupt transition is found at around $V=3.5 \mathrm{~V}$. We believe that in this case, the region under the tip was oxygen deficient and therefore oxygen vacancies were present. Thus, by applying a positive bias oxygen incorporation is favoured upon the application of the electric field to achieve a lower resistance state than the initial. Then, the sweep continues towards negative bias and back to $0 \mathrm{~V}$ (branches 3 and 4). A HRS state is induced and retained down to 0V. A second positive bias sweep is applied and should restore the original LRS. However, the induced LRS presents a lower current value than the original pristine in both cases. This can be explained in terms of a non-fully oxygen incorporation and/or oxygen vacancies rearrangement within the region below the tip upon the application of the electric field. The LRS generated after the oxygen incorporation is well distinguishable from the pristine state. We have not been able to ascribe particular I-V sweep behaviours to specific features of the film surface. Oxygen concentration seems to slightly vary randomly at the local scale, as it will be later demonstrated by Scanning Tunnelling Microscopy studies.

\subsubsection{Micrometric scale HRS areas induced by C-AFM}

One of the main advantages offered by an AFM to study RS phenomena is its versatility to study local inhomogeneities related to RS effect and to create nano- and micrometric HRS arrays. By means of a XY closed loop system, which enables to have a fine control over the tip position, we have written an array of resistance $2 \mathrm{D}$ 
maps at different resistance states, induced at different negative bias. On a pristine $25 \mathrm{~nm}$ LSMO film surface, six squares of $5 x 5 \mu \mathrm{m}^{2}$ were scanned with the C-AFM tip at $V_{\text {bias }}=\{-1,-2 \ldots-6 V\}$. HRS regions are then induced at a micrometric scale. After this process, a $20 \times 20 \mu \mathrm{m}^{2}$ topography and reading resistance maps were acquired. Low bias (1V) was applied for reading out of the generated HR regions to prevent any modification of the resistive state. An scheme of the whole process is presented in figure 3.5 (a), while (b) shows the final readout of the topography and (c) the resistance of the modified area.

a)

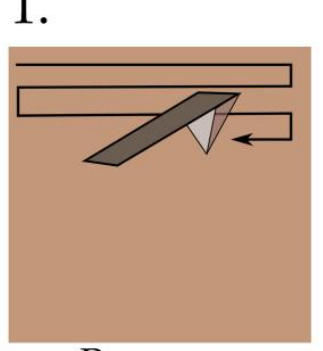

Pre-scan

Low voltage $\mathrm{V}_{\text {bias }}=1 \mathrm{~V}$
2.

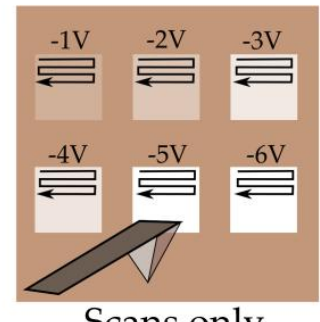

Scans only

in selected regions
3.

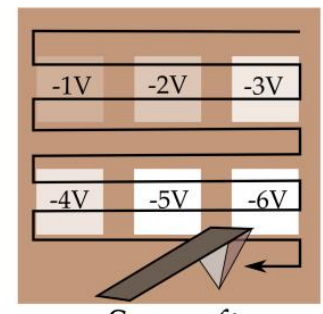

Scan after

inducing RS-MIT

Low voltage b)

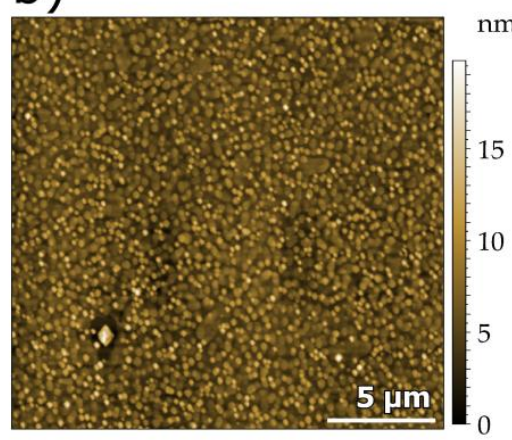

c)

$\mathrm{V}_{\text {bias }}=1 \mathrm{~V}$

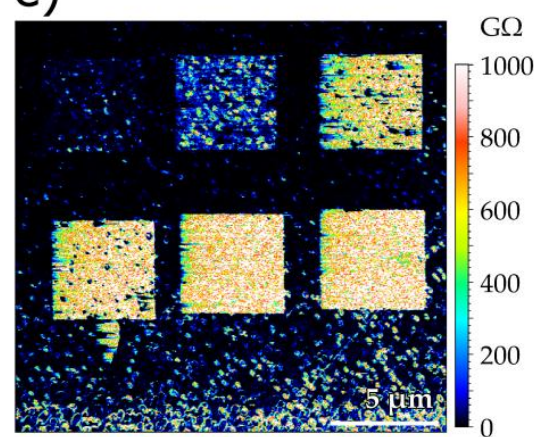

FIGURE 3.5: HRS micrometric regions were induced in a $25 \mathrm{~nm}$ LSMO thin film. (a) Diagram showing the protocol to generate and read the array. (b) Topography and (b) resistance map of the resulting resistance array

The resistance map shows clear contrast differences between the pristine and the induced HR areas. The higher the $\mathrm{V}_{\text {bias }}$, the more resistive the region appears whereas no topographical modifications are observed in any of the cases. In addition, the change in the resistance is produced below the tip, thus demonstrating that the RS effect occurs in the vertical direction in the region where the tip scans, and not laterally towards the counterelectrode.

Note that the resistance values obtained in the maps do not represent an absolute measurement of the intrinsic resistance of the material since the contact resistance might be large and difficult to determine [250, 251]. However, relative changes in the resistance within the same image can be quantitatively evaluated. To do so, first an average value of each HR square is calculated, $R_{H R S}$. Then, all the HR areas are subtracted from the original $R$ map and an average value of the surrounding pristine region is obtained, $R_{\text {prist }}$. By dividing each $R_{H R S}$ by the overall $R_{\text {prist }}$ value, 
a $R_{H R S} / R_{\text {prist }}$ ratio as a function of the $V_{\text {bias }}$ used to induced the HRS is obtained. These ratios are shown in figure 3.6.

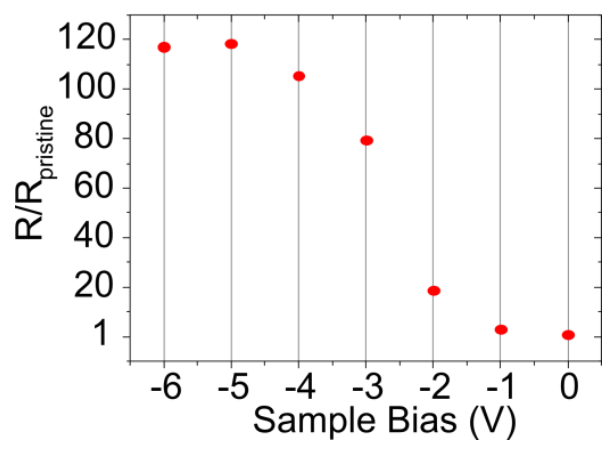

FIGURE 3.6: Ratio between the pristine state and the induced high resistance states as a function of the $V_{\text {Bias }}$ employed to generate the HRS.

A significant increase in the resistance ratio is found for $-5 V<V_{\text {write }}<-2 \mathrm{~V}$, whereas a saturation seems to appear for $V_{\text {write }}<-5 V$ and $V_{\text {write }}>-1 V$. This experiment further confirms the multilevel switching capabilities of these perovskite oxide systems. We believe that by increasing the magnitude of the applied field, larger exchange of $\mathrm{O}^{2-}$ ions from the LSMO crystal structure with ambient occurs thus reading a gradually higher resistance state.

To restore back the LRS in any of the HRS regions, they should be scanned at a proper positive voltage. In this way, oxygen incorporation in the crystal structure of the film is achieved. The experiment in figure 3.7 shows the resistance map of a partially restored LRS in the HRS square induced at $V=-2 \mathrm{~V}$. Only half of the $5 \times 5 \mu m^{2}$ square HRS was scanned at $\mathrm{V}_{\text {bias }}=+3 \mathrm{~V}$.Then, a resistance map of the region is acquired at low bias $(1 \mathrm{~V})$.
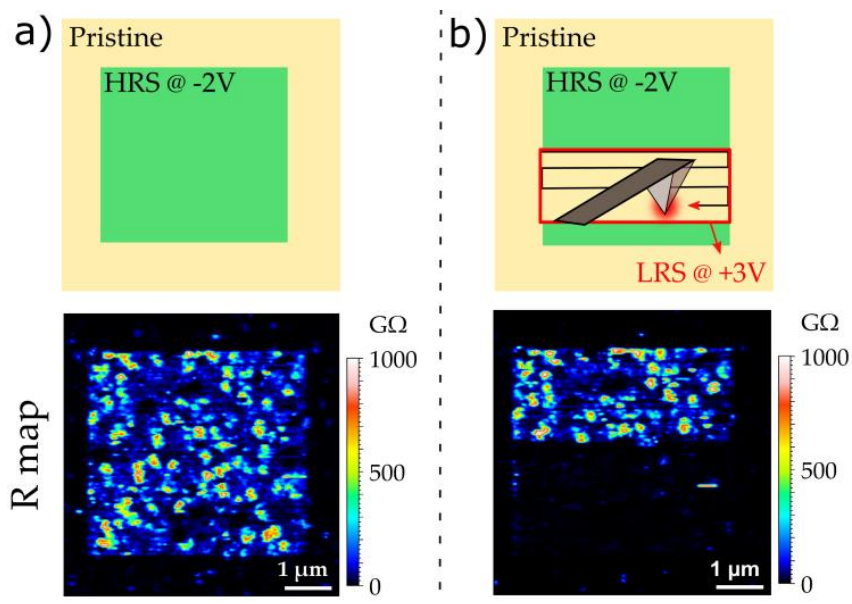

FIGURE 3.7: (a) Zoom in the HRS generated by scanning at $-2 \mathrm{~V}$. (b) Resistance readout at low bias after inducing a LRS at $+3 \mathrm{~V}$ in half of the HRS square.

Upper diagrams in figure 3.7 shows the film in the generated HRS (a) and the process to restore the LRS (b). Resistance maps at the bottom show the HRS (a) and the partially restored LRS (b). The part of the HRS square that has been scanned at $+3 \mathrm{~V}$, shows no contrast difference with the surrounding pristine state. Therefore, 
a LRS is recovered (on a microscopic region) by a positive bias scan on a previously generated HRS.

\subsubsection{Volume Resistive Switching in LSMO thin films}

The adaptability of the AFM to move with very fine precision in the $X-Y$ directions allow to define any kind of feature at will. In this section, a volume resistive switching mechanism will be demonstrated. To do so, cylindrical shape HRS areas will be induced in the films by drawing rings at a certain voltage, in a similar way as shown in previous section and reported in [50,51]. Along this thesis, we extend these previous studies to other metallic perovskite materials, as it will be shown in here ( $\mathrm{La}_{1-x} \mathrm{Sr}_{x} \mathrm{MnO}_{3}$ family compounds) and in next chapter (YBCO and $\mathrm{RENiO}_{3}$ ). For completeness, we present in this section experiments performed in $\mathrm{La}_{0.7} \mathrm{Sr}_{0.3} \mathrm{MnO}_{3}$ films of different thicknes. Notice that these experiments are carried out in a top-top configuration (see chapter 2 ) and the read out of the inner part of the ring is performed by placing the tip on the inner area of the ring whereas the counter electrode is placed outside of the ring.

a)

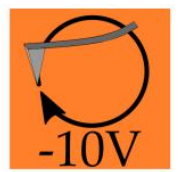

b) $22 \mathrm{~nm}$ thick

C) $10 \mathrm{~nm}$ thick
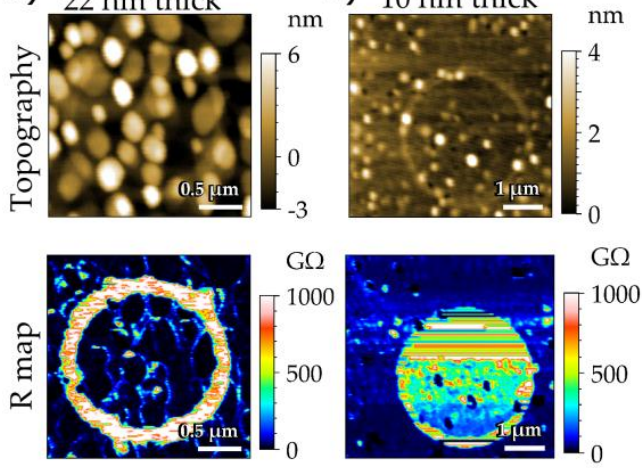

FIGURE 3.8: (a) Scheme indicating how a HR ring is generated at $-10 \mathrm{~V}$. Topography and resistance maps for a (a) 22 and (b) $10 \mathrm{~nm}$

LSMO thin film after creating the HRS ring.

In a first step, two rings were drawn with a $V_{\text {bias }}=-10 \mathrm{~V}$ in two films of thickness 22 and $10 \mathrm{~nm}$ (figure 3.8 (b) and (c), respectively). The reading resistance maps show that, when the $-10 \mathrm{~V}$ ring is drawn in the $22 \mathrm{~nm}$ film, the ring area scanned by the tip has been transformed into the HRS. When the same ring is drawn in the $10 \mathrm{~nm}$ film, we observed that not just ring but also the inner part of it (which has not been modified by the tip), also appears to be in the HRS. In the thicker film, not all the volume under the tip is transformed into the HRS when the $V_{\text {bias }}=-10 \mathrm{~V}$ ring is used, and therefore the inner part of the ring is electrically connected to the outer pristine film. However, when the thickness of the film is decreased and the same high voltage $V_{\text {bias }}=-10 \mathrm{~V}$ is used to draw the ring, the HRS reaches the bottom surface until the insulating substrate, thus creating a HR cylinder wall, that impedes connectivity between the tip and the counter electrode. Therefore, metallic non-modified regions are topologically isolated in this case. 
In a second step, if a tiny part of the HR wall generated in the $10 \mathrm{~nm}$ film is scanned at high enough and opposite bias $\left(V_{\text {Bias }}=+10 \mathrm{~V}\right)$ to induce the LRS. Then a new resistance map is acquired (figure 3.9), and a fully metallic region in the inner part of the ring is observed. By inducing a LRS in a small portion of the wall, the inner non-modified region and the outer pristine film are electrically connected again.
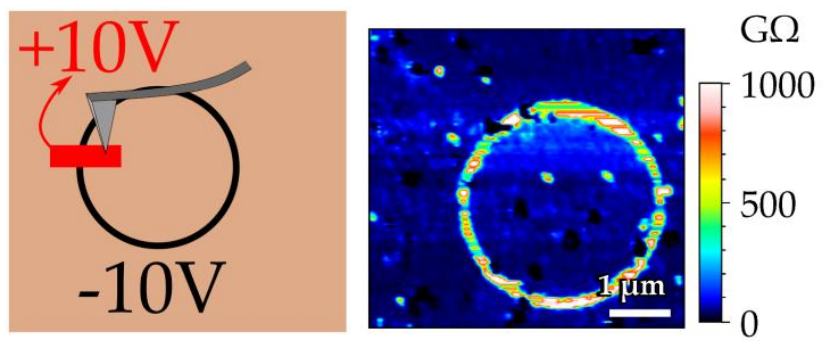

FIGURE 3.9: Inducing a LRS in the HRS wall by applying a $+10 \mathrm{~V}$ scan in the red area. Resistance map acquired at low bias after generating the LRS in the wall.

A second experiment reported in this section reproduces the same phenomenology described above, in a $10 \mathrm{~nm}$ film, with rings drawn at different voltage amplitudes. Figure 3.10 shows two rings were scanned at $-6 \mathrm{~V}$ and $-10 \mathrm{~V}$, respectively. The resistance map obtained in this case, after drawing the rings, shows again the two different scenarios described above. In the $-6 \mathrm{~V}$ case (left), the region where the HRS was induced corresponds to the region scanned by the tip. In contrast, in the $-10 \mathrm{~V}$ case, the inner non-modified region appears to be in the HRS. As in the case described above, the generated HRS has reached the insulating substrate, and therefore the conduction from the inner part of the cylinder to the counterelectrode is prevented by the presence of the HRS cylinder wall.
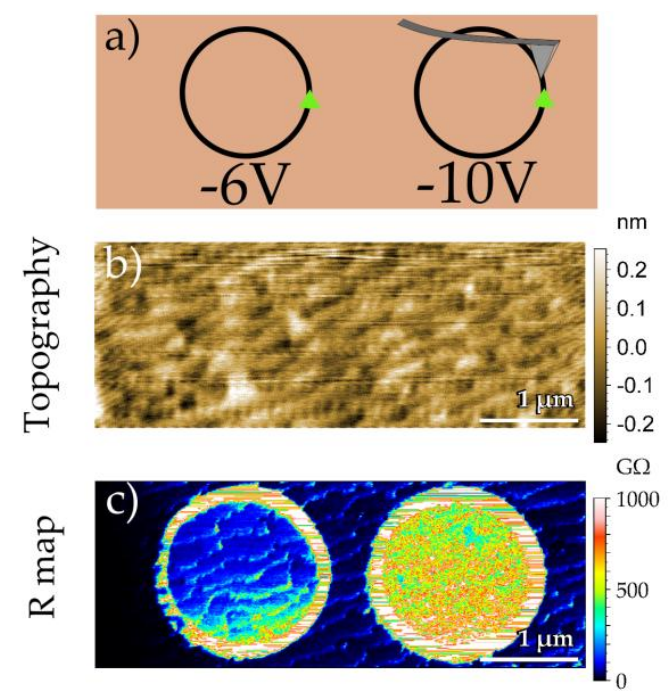

FIGURE 3.10: (a) Diagram, (b) topography map and (c) resistance map of two HRS rings generated at -6 and $-10 \mathrm{~V}$ in a $10 \mathrm{~nm}$ LSMO thin film.

For completeness, a small portion of the HR cylinder was transformed back into the LRS by applying a $\left(V_{\text {bias }}=+10 \mathrm{~V}\right)$. After that, the read out of the region is performed at low bias (1V). Similarly to the thickness dependence case explained 
above, the inner non-modified region and the outer pristine film are now electrically connected, as shown in figure 3.11.
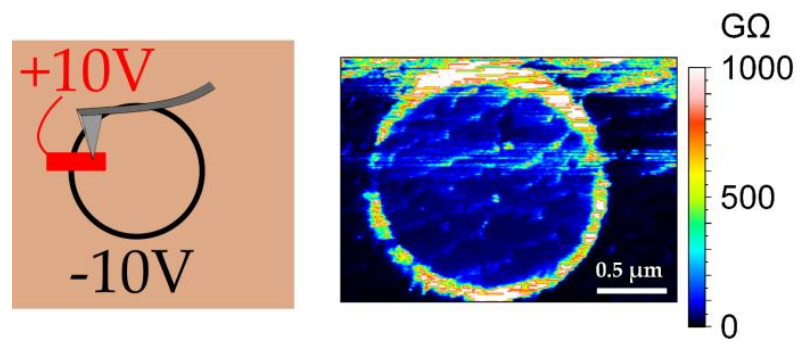

FIGURE 3.11: Generating a LRS in the HRS wall by scanning a small portion of the wall at $+10 \mathrm{~V}$. Resistance map obtained at low bias

These experiments in which the thickness of the film and voltage amplitude were varied, provide strong evidences that a Volume Resistive Switching (VRS) mechanism is occurring. We demonstrate that the whole material volume under the tip can be homogeneously transformed into a HRS, instead of a filamentary conduction process. Furthermore, it also suggests that the oxygen depletion is occurring gradually through thickness since at $V_{\text {bias }}=-6 V$, although a clear HRS sign is achieved, there is electrical conduction between the inner surface of the ring and the counter electrode. Instead, for $V_{\text {bias }}=-10 \mathrm{~V}$, metallic non-modified regions are topologically isolated in the case of the $10 \mathrm{~nm}$ film. A schematic explanation of this effect is shown in figure 3.12.

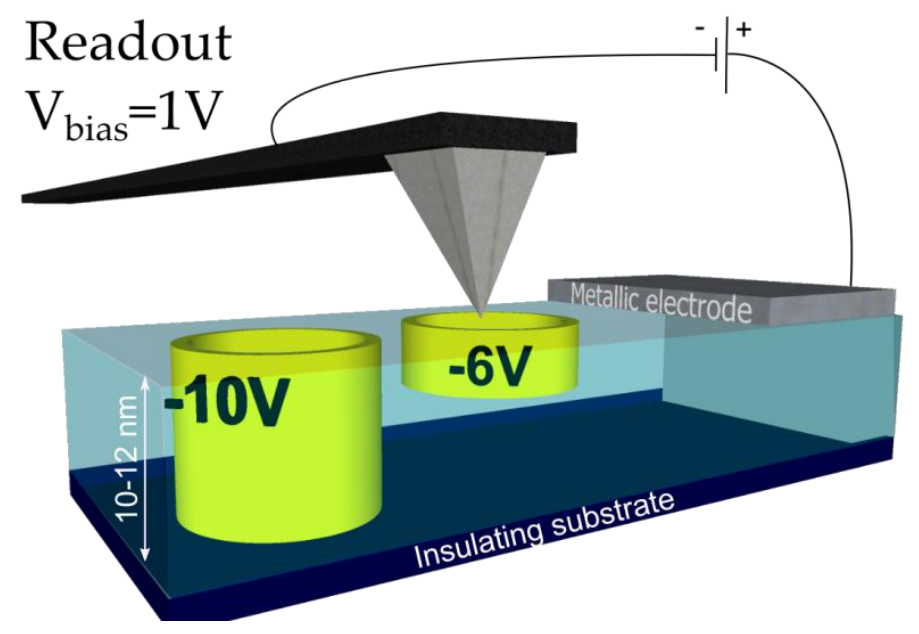

FIGURE 3.12: Schematic illustrating the Volume Switching character of the MIT-RS behaviour as an intrinsic property of the system. .

The suggested Volume RS (VRS) mechanism is different in nature from the filamentary or interfacial types early reported. VRS might be an intrinsic property of metallic perosvkite complex oxides showing metal-insulator transition. We believe that it is the MIT of these systems what induces the RS effect in these films upon small variations in the carrier density induced by the applied electric field. Starting from a LRS, oxygen excorporation occurs at negative bias inducing a valence change in the transition metal due to the creation of oxygen vacancies in the system, which simultaneously produces a decrease of the carrier density, thus crossing the MIT line and entering into a HRS regime. At positive bias, oxygen incorporates into the crystal structure and annihilates oxygen vacancies. The reverse valence 
change occurs accompanied by an increase of the carrier density, restoring the LRS upon crossing back the MIT. We would like to emphasize the link between the RS effect of these materials and their MIT, which at least at high bias, is fully crossed, as it will be shown in section 3.3. The crossing of the MIT line is not a "conditio sine qua non" to generate a higher resistance state. However, an enhanced performance is expected if the MIT line lies close to the operating temperature. Our experiments demonstrate that the volume under the tip can be progressively driven into a HRS upon increase of the voltage amplitude. This change is highly directional and occurs vertically below the tip as. The suggested Volume RS should be therefore understood in counter opposition to interface RS, which would be localized in the few units cells close to one of the electrodes. In contrast, the full thickness of the film $(10 \mathrm{~nm})$ can be transformed here into the HRS.

\subsubsection{Dependence of Sr content on the RS properties}

The tunability of the metal-insulator transition in $\mathrm{La}_{1-x} \mathrm{Sr}_{x} \mathrm{MnO}_{3}$ can be achieved by introducing chemical doping [73], varying thickness[159], strain [252], inducing the presence of oxygen vacancies[253] or upon the application of an electric field, as shown along this chapter. We show in the previous chapter that a modification of the Sr content of the LSMO precursor solution, in stoichiometric proportion, leads to a shift in the MIT and resistivity. The lowest resistivity and highest $\mathrm{T}_{\mathrm{MIT}}$ is found for $x=0.3$ films whereas decreasing the Sr content shifts the MIT towards lower temperatures. We will follow this approach to properly engineer the growth of the material tuning its electronic properties in such a way that the MIT lies at different temperatures [78]. Ideally, a material designed with a MIT close to the operating temperature could easily undergo a resistance switch, with a small variation of the carrier density. For RS applications, this should lead to low operating voltages and even higher resistance ratios. The diagram shown in in figure 3.13 shows an schematic view of the explanation stated here.

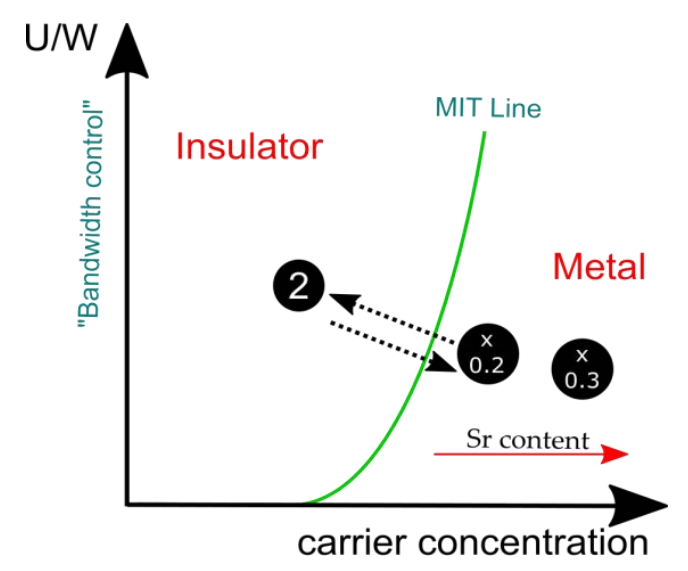

FIGURE 3.13: Bandwidth control vs band-filling control schematic.

Two different Sr contents are schematically represented.

As it was shown in chapter 2, LSMO films with different Sr content showed a clear shift in the MIT. Figure 3.14 shows the $\mathrm{T}_{\text {MIT }}$ dependence on the formal Mn valence state, $\mathrm{Mn}_{\mathrm{FV}}$, observed in our films and in bulk samples [73]. Manganites are mixedvalence systems consisting of a mixture of $M n^{+3}$ and $M n^{+4}$, with the $M n^{+3} / M n^{+4}$ 
ratio equal to $1 /(1-x)$. The composition of the compound can be expressed as:

$$
L a_{1-x} S r_{x}\left(M n_{1-x}^{3+} M n_{x}^{4+}\right) O_{3}=L a_{1-x} S r_{x} M n_{F V}^{(3+x)+} O_{3}
$$

Thus, the nominal oxidation states, $\mathrm{Mn}_{\mathrm{FV}}$ for the considered $\mathrm{Sr}$ contents $(x=$ $\{0.15,0.2,0.3\})$ are $+3.15,+3.2$ and +3.3 , respectively. This nominal value was determined by assuming that the change in the $\mathrm{Sr}$ content in the precursor solution determines the valence state. However, local inhomogeneities leading to a phase separation at the nanoscale could also occur in some cases [84, 254, 255]. At lower Sr doping levels $(x=0.15)$, we observed that the $\mathrm{T}_{\text {MIT }}$ of our films occurs at much higher temperatures than in the bulk. This deviation from the expected bulk value makes us to conclude that we cannot discard non-stoichiometric oxygen content or a predominant role of biaxial strain in films with lower Sr content. Therefore, we chose $\mathrm{La}_{0.8} \mathrm{Sr}_{0.2} \mathrm{MnO}_{3}$ and $\mathrm{La}_{0.7} \mathrm{Sr}_{0.3} \mathrm{MnO}_{3}$ films to perform quantitative studies of the role of the initial doping content in the RS effect.

FIGURE 3.14: $\mathrm{T}_{\mathrm{MIT}}$ as a function of the Mn formal valency in our LSMO films and in bulk samples [73].

The same experiment presented above, where HRS cylinders were induced at different negative bias, was conducted in a $10 \mathrm{~nm} \mathrm{La}{ }_{0.8} \mathrm{Sr}_{0.2} \mathrm{MnO}_{3}$ thin film. Figure 3.15 shows the resistance map obtained at $1 \mathrm{~V}$ after two rings were drawn at $-10 \mathrm{~V}$ and $-6 \mathrm{~V}$, respectively (figure 3.15 (a)). In the $-6 \mathrm{~V}$ case, only the area scanned by the tip (the contour of the ring) appears in a HRS. In contrast, in the $-10 \mathrm{~V}$ case the inner region also seems to be in an insulating state, even though it was not modified by the tip. In this last procedure, the same electrically driven transition linked to the MIT described above transforms the sample into HRS through the film thickness. The created HRS cylinder walls blocks the current when the tip is placed in the inner part of the ring during the readout and therefore, the image shows an apparent insulating behaviour. In the right image, a tiny portion of the HRS wall was scanned at $V_{\text {Bias }}=+10 \mathrm{~V}$ to locally restore the LRS. After that, the readout resistance map is acquired. Since the HRS ring has been opened, the inner non-modified region is not topologically isolated anymore and therefore electrical conductivity between the tip and the counterelectrode is observed.

With these measurements, no difference between the $x=0.2$ and $x=0.3$ can be detected. We next study the influence of the Sr content on the I-V characteristics. For that, I-V sweeps were acquired under the same conditions in $10 \mathrm{~nm} \mathrm{La} \mathrm{La}_{1-\mathrm{x}} \mathrm{Sr}_{\mathrm{x}} \mathrm{Mn}_{3}$ thin films with $x=\{0.3,0.2\}$. To minimize the effect of local inhomogeneities [84, 
a)

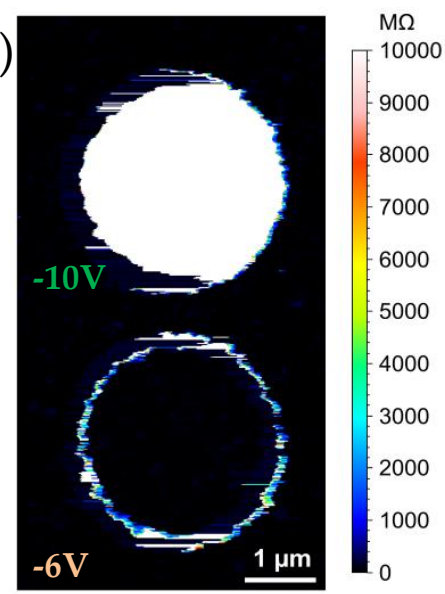

b)

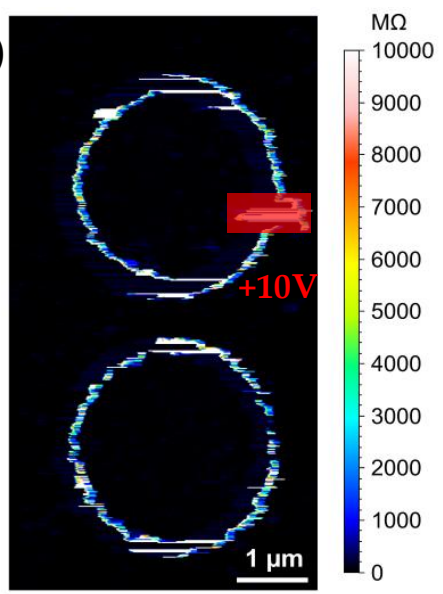

FIGURE 3.15: (a)Resistance map at $1 \mathrm{~V}$ after inducing $\mathrm{HR}$ rings at $-10 \mathrm{~V}$ and $-6 \mathrm{~V}$ in a $10 \mathrm{~nm} \mathrm{La}_{0} .8 \mathrm{Sr}_{0} \cdot 2 \mathrm{MnO}_{3}$ thin film.(b) Resistance map at $1 \mathrm{~V}$ after restoring the conductance with a scan at $+10 \mathrm{~V}$ in the $\mathrm{HR}$ wall previously generated at $-10 \mathrm{~V}$ demonstrating the volume RS in this compound.

254, 255], a statistical study of more than $100 \mathrm{I}-\mathrm{V} /$ film was performed. In a similar way, the effect of possible oxygen vacancies in the region of the tip was minimized by performing first a sweep towards positive bias, as it was described in previous section (figure 3.4). Briefly, the protocol applied was:

1. Sweeps 1\&2: $\mathbf{0 V} \rightarrow+5 \mathrm{~V} \rightarrow \mathbf{0 V}$ : As it was stated before, a first positive sweep is performed to incorporate more oxygen in case the region under the tip is an oxygen-deficient area. In this case, a small transition occurs to incorporate oxygen intro the structure. Otherwise, the back and forth sweeps are reversible.

2. Sweeps 3\&4: $0 \mathrm{~V} \rightarrow-5 \mathrm{~V} \rightarrow \mathbf{0 V}:$ In this branch, the LRS $\rightarrow$ HRS transition is produced as it was explained before. Eventually the MIT line is crossed and the system remains in an insulating HRS down to $0 \mathrm{~V}$.

3. Sweeps $5 \& 6: 0 \mathrm{~V} \rightarrow+5 \mathrm{~V} \rightarrow \mathbf{0 V}$ : By increasing the bias voltage towads $+5 \mathrm{~V}$, an abrupt $\mathrm{OFF} \rightarrow \mathrm{ON}$ transition occurs at a given $\mathrm{V}_{H R S \rightarrow L R S}$. This threshold voltage will be the main parameter analyzed in the following.

Figure 3.16 shows several full I-V sweeps as described in the protocol for both $\mathrm{Sr}$ content cases. Similar I-V characteristics are observed for the two films. In both cases, a bipolar RS behaviour is found whose origin has been explained above. Huge resistance ratios of $10^{3}-10^{5}$ are generated in both systems. In the negative branch, a smooth $\mathrm{ON} \rightarrow \mathrm{OFF}$ transitions occur, whereas in the positive branch a much abrupt $\mathrm{ON} \rightarrow \mathrm{OFF}$ transition is observed. Differences in this threshold voltage, $\mathrm{V}_{\mathrm{HRS} \rightarrow \mathrm{LRS}}$, of the two systems are observed. A cumulative probability study was performed for an statistical analysis of more than $100 \mathrm{I}-\mathrm{V}$ curves (figure 3.16 (b). Under the same applied electrical stress in both systems, a similar resistance ratio HRS/LRS $10^{3}-10^{5}$ is generated. In the case of $x=0.2$ a clear shift of the $\mathrm{V}_{\mathrm{HRS} \rightarrow \text { LRS }}$ towards lower voltage values is found. From our point of view, this fact is consistent with the proposed MIT-induced RS for these compounds. Therefore, voltage inhomogeneities are smaller and a more abrupt threshold is found for the $x=0.2$ case. We could not find a correlation between the RS behaviour and any 
topographic feature. Therefore, we believe that the differences observed in this experiment are related to intrinsic electronic inhomogeneities of the films.

a)

b)

FIGURE 3.16: (a) I-V characteristics in $10 \mathrm{~nm} \mathrm{La} \mathrm{La}_{1-\mathrm{x}} \mathrm{Sr}_{\mathrm{x}} \mathrm{Mn}_{3}$ thin films with $x=0.3$ and $x=0.2$. (b) Cumulative probability plot derived from the I-V statistical study.

Thus, we have shown that the RS parameters of the $\mathrm{La}_{1-x} \mathrm{Sr}_{x} \mathrm{MnO}_{3}$ family compounds can be tuned by means of chemical doping. We have shown that the modification of the $\mathrm{Sr}$ content modifies the carrier concentration in such a way that MIT gets closer to the operating temperature. In that situation $(x=0.2)$, lower threshold voltages are found. We believe that further optimization process in this direction, all the potentiality of the material as a memristive element could be fully exploited.

\subsection{Ex-situ characterization of LSMO thin films by STM/S}

As discussed in chapter 2, STM is a very powerful technique to reveal local features of the Local Density of States of the material (LDOS) by performing tunnel spectroscopic experiments.

In particular, manganites were heavily studied by STM in the last 20 years. Mainly, efforts were made to elucidate the existence of microscopic phase separation [84, 254-258] and to understand the fundamental transport properties (CMR and MIT) of these compounds [257, 259-263].

In the field of RS, STM is not a widely used technique, since most of the materials are insulators, and therefore not suitable for STM studies. However, very recently investigations in which STM is used to induce and characterize RS behaviour in metallic $\mathrm{SrRuO}_{3}$ thin films[264], semiconducting materials $\mathrm{Nb}: \mathrm{SrTiO}_{3}$ single crystals [265] or insulating binary oxides [266] or even Mott insulators [80] were reported.

In this section, we provide STM/S ex-situ characterization of pristine and HRS regions induced by C-AFM at different negative bias in $10 \mathrm{~nm} \mathrm{La} \mathrm{La}_{1-\mathrm{x}} \mathrm{Sr}_{\mathrm{x}} \mathrm{MnO}_{3}$ thin films.

\subsubsection{Sample preparation and measurement procedure}

First, let us explain the procedure followed to generate the large HRS at ICMAB facilities and the STM/S measurement protocol at the University of Geneva. 


\section{Sample preparation at ICMAB}

The localization of micrometric HRS areas induced by C-AFM in a $5 x 5 \mathrm{~mm}^{2}$ sample with precision might present a major drawback to perform ex-situ STM measurements, since the STM tip should be positioned in the micrometric region which was previously modified. To address this issue, a lithography process is carried out to define a grid of $60 x 60 \mu \mathrm{m}$ equally spaced where $50 \mathrm{~nm}$ thick Au marks were sputtered. These Au squares act as a guide reference to precisely locate the STM tip afterwards at will. Figure 3.17 (a) shows an optical micrograph of one of the samples after this process. The pristine $60 x 60 \mu \mathrm{m}$ regions in between the Au reference marks (black squares in the OM image) are the spots where the RS was induced by C-AFM. The AFM tip was situated in the middle point in the chosen region and the scan size was set to exceed the $60 x 60 \mu \mathrm{m}$ scan area. Two different $\mathrm{V}_{\text {bias }}$ were used to create progressively higher resistance state regions in the films. In these experiments, $10 \mathrm{~nm}$ samples of $x=\{0.15,0.2,0.3\}$ were used, while the bias voltages chosen were $\mathrm{V}_{\text {Bias }}=\{-5 \mathrm{~V},-7 \mathrm{~V}\}$. For comparison, the pristine state was also studied. Figure 3.17 (b) shows an example of such a large HRS region described obtained at $1 \mathrm{~V}$ after inducing the HRS at a $\mathrm{V}_{\text {Bias }}=-5 \mathrm{~V}$ in the $10 \mathrm{~nm} \mathrm{La}{ }_{0.8} \mathrm{Sr}_{0.2} \mathrm{MnO}_{3}$ film. In the image, a large HRS square (white) is observed surrounded by the pristine film (dark blue).

a)

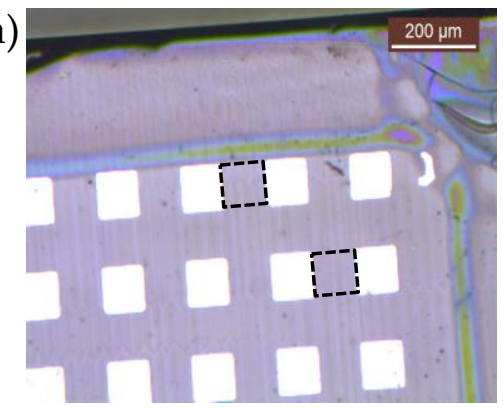

b)

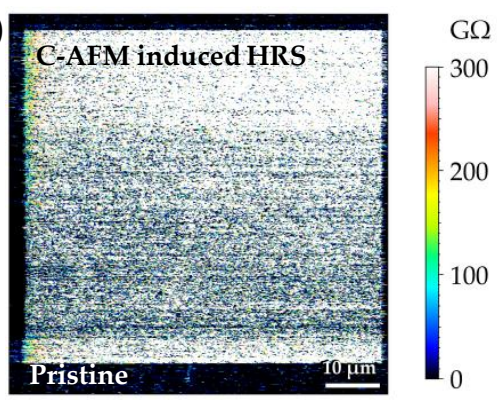

FIGURE 3.17: (a) Optical image of one of the films after the sputtering $\mathrm{Au}$ reference marks. In black, the regions in between the references were HRS were induced. (b) Resistance map of a large $60 x 60 \mu m^{2}$ HRS induced at $\mathrm{V}_{\text {Bias }}=-5 \mathrm{~V}$ in the $10 \mathrm{~nm}$ $\mathrm{La}_{0.8} \mathrm{Sr}_{0.2} \mathrm{MnO}_{3}$.

\section{Measurement procedure at University of Geneva}

Figure 3.18 shows a schematic representation of the samples used. The different elements in the image and the protocol established to measure are described in the following:

1. To locate the tip on top of one the desired regions (pristine, HRS performed at $-5 \mathrm{~V}$ or HRS performed at $-7 \mathrm{~V}$ ) we used the micron-step stage ( $\mathrm{XY}$ control) and the lateral-side binoculars. Illustratively, these $60 x 60 \mu \mathrm{m}^{2}$ areas indicated with red squares in the scheme.

2. As defined in chapter 2, all the measurements were performed under the same conditions: In air with a $\mathrm{V}_{\text {Bias }}=800 \mathrm{mV}$ and a tunnelling current set point of $\mathrm{I}_{\mathrm{SP}}=200 \mathrm{pA}$. The tip is brought to the tunnelling regime in those conditions. The scan area is set to $800 \mathrm{~nm} \times 800 \mathrm{~nm}$ and several topography 
images are obtained in the same area for a better stabilization prior to any spectroscopic measurement.

3. After achieving stable topography images, the spectroscopic experiment is carried out. This measurement acquires simultaneously topography and spectroscopic data (I-V sweeps at every pixel of the image). Each map takes 8-12 hours to be collected. It is performed in an automatic mode.

4. After these data acquisition, the tip is placed to another area within the same resistance state region and steps 2 and 3 are repeated to acquire more spectroscopic maps micrometers away with the aim of perform statistical analysis.

5. Step 4 is repeated several times for each resistance region (indicated as green squares in the drawing) and for each sample to check homogeneity and reproducibility of the measurements.

6. The data was analysed with a specific software developed in the group at the University of Geneva. Zero Bias Conductance (ZBC, see chapter 2) maps are obtained. Then, the histogram of the $Z B C$ values distribution for each map is extracted. To compare between the different regions within a sample and in between samples an average $Z B C$ value for each region is required The analysis will be detailed along these chapter.

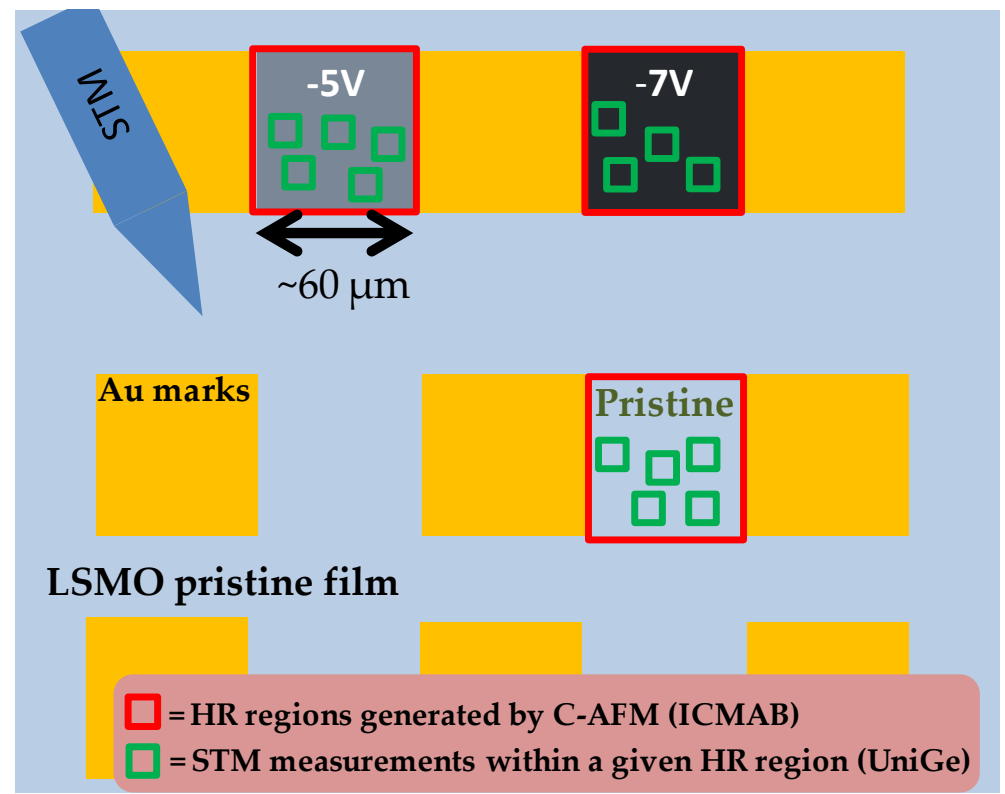

FIGURE 3.18: Schematic view of the LSMO samples with the HR regions (red squares) and the areas within these regions where the STM/S measurements were performed (green squares).

In the following, detailed results for all the experimental steps described above will be presented. First, a complete characterization of the three samples $(x=0.15$, 0.2 and 0.3 ) in their pristine state will be shown. Then, the study of the HRS regions in the $x=0.2$ sample will be exposed, and finally, a comparison between the $x=0.2$ and $x=0.3$ will be performed. 


\subsubsection{STM/S studies in the pristine state of $\mathrm{La}_{1-\mathrm{x}} \mathrm{Sr}_{\mathrm{x}} \mathrm{MnO}_{3}$ thin films}

First, the characterization of the pristine state for the three samples with different $\mathrm{Sr}$ content will be here presented. Several spectroscopic measurements were carried out in different $800 \times 800 \mathrm{~nm}^{2}$. As a reminder, each map is composed of a complete set of I-V curves (1 curve/pixel). A custom-made software developed at the group at the University of Geneva was employed for data processing. The numerical derivative is calculated from the raw I-V data to obtain the conductance curves $G=d I / d V$. Then, a map with the $\mathrm{G}$ curves is evaluated at $0 \mathrm{~V}$, i.e., the ZBC map. This procedure is applied for each sample. An example for each of the samples is shown in figure 3.19 .
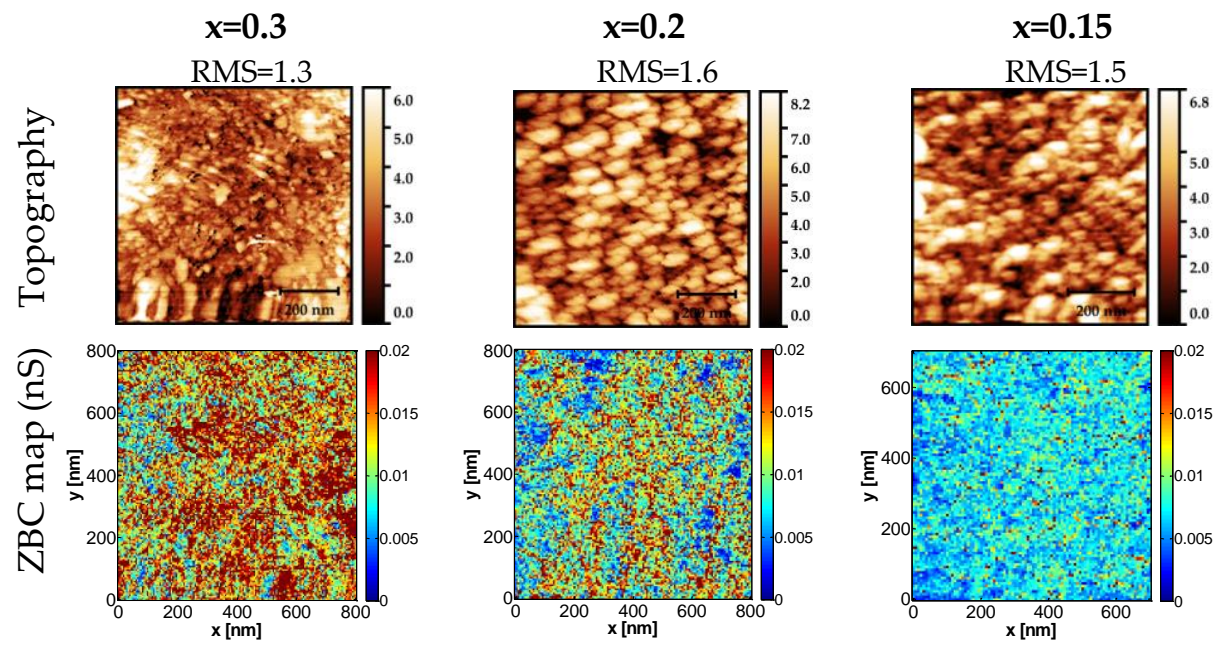

FIGURE 3.19: Topography (first row) and ZBC maps (second row) for the $10 \mathrm{~nm}$ LSMO films of $\mathrm{x}=0.3,0.2$ and 0.15 (first, second and third column respectively).

A granular morphology is observed in the three films with no relevant changes among them and similar root mean square (RMS) values $(1.3-1.6 \mathrm{~nm})$. However, strong differences are clearly identified in the ZBC maps. A continuous shift from red (higher $\mathrm{ZBC}$, higher conductivity) towards light blue (lower $\mathrm{ZBC}$, lower conductivity) is found when decreasing the Sr content of the film. In each case, the ZBC values shows a homogeneous distribution and it cannot be correlated with any specific topographic feature. In figure 3.20 the corresponding histograms of the ZBC maps were extracted. A clear shift of the whole spectra towards lower $Z B C$ is found as the $S r$ content is decreased. $G(V)$ curves are proportional to the LDOS, and therefore, $\mathrm{ZBC}=\mathrm{G}(0)$ is proportional to the LDOS at the Fermi Level, i.e., its conductivity. The lower Sr content, the lower the $\mathrm{Mn}^{+4} / \mathrm{Mn}^{+3}$ ratio, and therefore less conductivity in the I-V curves and smaller ZBC values are found. This is consistent with the trend observed in the $\rho(T)$ measurements shown in chapter 2 .

After this first characterization an average I-V curve is obtained for each map by averaging the full set of I-V curves measured. Since the STS maps were set to $100 x 100 p x^{2}$ size, $10^{4} \mathrm{I}-\mathrm{V}$ curves are averaged to obtain a mean I-V response for the whole $800 x 800 \mathrm{~nm}^{2}$ area. Despite the loss of spatial resolution of the analysis, this approach permits to extract average response of the full pristine region, and therefore, a first-order characterization of the DOS of the material in a given resistive state avoiding local phenomena such as precipitates, local variations in the oxygen content or inhomogeneous Sr cation distribution. 
FIGURE 3.20: Histograms extracted from the ZBC maps shown in figure 3.19.

Figure 3.21 shows the average I- $\mathrm{V}$ curves and their numerical derivatives $(\mathrm{G}$, conductance curves) for the three ZBC maps of figure 3.19. The conditions imposed in the experiment forces the three I-V average curves to cross at $800 \mathrm{mV}\left(\mathrm{V}_{\text {bias }}\right)$ at the $\mathrm{I}_{S P}=200 \mathrm{pA}$ and changes in the I-V response are expected to appear at lower bias. The G-V curves are parabolic. The parabolic shape of the conductance curves is essentially a property of the tunnelling barrier [267, 268]. In all the cases,there is a finite value of the $\mathrm{ZBC}$ and a clear shift from the more metallic $(x=0.3)$ to the less metallic $(\mathrm{x}=0.15) \mathrm{LSMO}$ pristine states behaviour is observed. For a given $\mathrm{V}_{\text {Bias }}<800 \mathrm{mV}$, less tunnelling current is read out when decreasing the Sr content of the film. In the $G(V)$ graphs, $G(0)=Z B C$ shows accordingly the same trend. The decrease in the $\mathrm{ZBC}$ is observed again in this plot. In addition, a shift in the minima of $\sim 25 \mathrm{mV}$ is observed in all the cases. By performing I-V sweeps in the forward and backward direction, the same shift is observed, thus ruling out the artefact character of this feature. In her thesis [269], Dr. Lina Chen thoroughly studied the origin of the bias shift in PLD-LSMO thin films. It is explained by the presence of surface polarization states due to Sr segregation close to the surface. The effect is more pronounced in ultrathin films, where strain effects arising from the STO substrate might contribute too. However, in our case, no substantial changes in the shift between the pristine and the HR states were found. Furthermore, we did not perform any experiments to further investigate the origin of the bias shift in our films, since it was out of the scope of our study. Therefore, we validate this approach to perform a semi-quantitative analyses of the electronic response of the films.

a)

b)

FIGURE 3.21: (a) Mean I-V pristine curves for the different $10 \mathrm{~nm}$ LSMO films. (b) Numerical derivative $G=d I / d V$ averaged from the maps shown in figure 3.19 . 
The $\mathrm{ZBC}$ values of these graphs are then extracted. As indicated in the measurement protocol, this same procedure is repeated several times for each sample (new area within the same resistive state region $\rightarrow \mathrm{STM} / \mathrm{S}$ measurement $\rightarrow$ Data processing $\rightarrow$ ZBC map $\rightarrow$ ) Average ZBC value) to obtain reasonable statistics and quantify more precisely the average response of the material in the pristine state. In this way, a total average value for the pristine state of each of the films is obtained by averaging the different average I- $V$ curves (and derived ZBC values) extracted from each of the maps. Typically, in each sample 5-8 maps in the pristine region were acquired. This total mean value would act as a reference value to compare the different samples and the different resistive states (within a same sample). In short, more than $5 \cdot 10^{4}$ were averaged to obtain a reference $\mathrm{ZBC}$ value of each film.

Figure 3.22 shows the ZBC values extracted from each single ZBC map measured in each of the samples. Although some deviations within the same sample are observed, a general trend can be uptaked. In the right image, the average values with the corresponding standard deviation is plotted as a function of the Mn formal valence, which can be nicely fit with a linear dependence. This linear fit will be of crucial importance to interpret the data of the HR states, as it will be explained later.

a)

b)

FIGURE 3.22: (a) ZBC value of all the measurements performed in the three samples in the pristine state. Each point represents the average value of the full data set obtained in the measurement. (b) Average values $\mathrm{ZBC}$ obtained from (a) for each sample as a function of the Mn formal valency of the sample. Dashed line is the linear fit to the data.

\subsubsection{STM/S of ex-situ generated HRS regions in $\mathrm{La}_{0.8} \mathrm{Sr}_{0.2} \mathrm{MnO}_{3}$ thin films}

The full characterization of the $e x$-situ generated high resistance states in the 10 $\mathrm{nm} \mathrm{La}{ }_{0.8} \mathrm{Sr}_{0.2} \mathrm{MnO}_{3}$ thin film is presented here. As shown in previous section, STS is a powerful technique to study the LDOS at the Fermi Level. By performing the same analysis in various induced HRS, we can gain insight into the electronic behaviour in such a resistance state and compare the tunnelling response of pristine/LRS and HRS. To do so, the same measurement protocol as in the previous section for the pristine state will be here applied to both HRS regions. As a reminder, we proceeded as follows: for a given generated HRS, several spectroscopic maps (at different locations within the HRS region) are acquired at the same conditions $\left(\mathrm{V}_{\text {bias }}, I_{\mathrm{SP}}\right)$ used in the pristine state data acquisition. Then, an average I-V response might be calculated for each map. Hence, a total mean I-V response for 
the generated HRS of each sample can be obtained by averaging these mean I-V sweeps of the different maps. A mean ZBC which characterizes that HRS is then straightforwardly derived.

Figure 3.23 shows three ZBC maps and their corresponding topography for each resistance state in the film (pristine, HRS generated at $-5 \mathrm{~V}$ and HRS generated at $-7 \mathrm{~V})$. In the three cases, the surface morphology present a grain-like structure with a grain size of 50-100 nm. Within our resolution limit, no evident changes in the topography were observed, besides a slight increase in the RMS as indicated in the figure. We cannot conclude whether this increase is a location-dependent phenomena or is arising from the induced RS effect. However, a clear contrast difference is detected in the ZBC map. A shift from red (higher ZBC values, higher conductivity) in the pristine state to a deep blue (lower ZBC values, lower conductivity) in the HRS regions is found. This is consistent with the increase of the resistivity of the HRS region. Also, the -7V HRS region presents a lower ZBC than the $-5 \mathrm{~V}$ HRS, as expected since we already demonstrated that at higher negative bias a deeper transformation in thickness is achieved and therefore, a higher resistance state is generated.
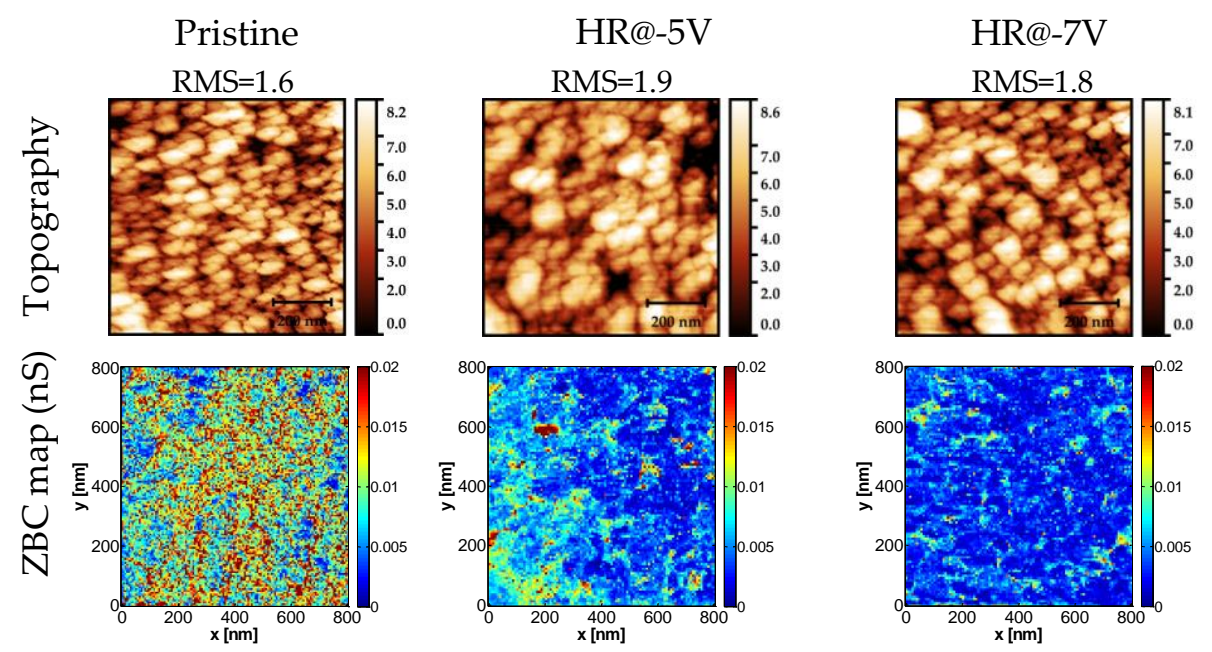

FIGURE 3.23: Topography (first row) and ZBC maps (second row) for the $10 \mathrm{~nm}$ LSMO films of $x=0.2$ in the pristine, $-5 \mathrm{~V}$ HRS and $-7 \mathrm{~V}$ HRS (first, second and third column respectively).

Remarkably, in none of the maps obtained in the measurements it was possible to correlate specific features of the ZBC map to any topographic detail, such as defects, pores or grain boundaries. This suggests a very homogeneous RS effect with no preferential sites where the RS could have taken place. For clarification the ZBC histograms have been extracted from the three ZBC maps and plotted in left image in figure 3.24. A complete shift in the distribution is found when comparing the pristine state and any of the induced HRS. This decrease in the ZBC is associated to a change in the Mn formal valence induced by the electric-field driven motion of oxygen vacancies and the associated decrease of the free charge carriers in the system.

As stated, several spectroscopic measurements were performed in different areas within the same resistive state region. From each map, an average ZBC value is obtained. Figure 3.24 shows the $\mathrm{ZBC}$ for all the measurements performed in this film in the pristine and in both HRS. The same trend presented in the maps above 
a)

b)

FIGURE 3.24: Histograms extracted from the ZBC maps shown in figure 3.23 .

is found. Then, all the points belonging to the same resistive state will be averaged to obtain a final $\mathrm{ZBC}$ value for each of the regions. In the next section, this analysis will be extended and generalized to the $\mathrm{La}_{1-\mathrm{x}} \mathrm{Sr}_{\mathrm{x}} \mathrm{MnO}_{3}$ samples with different $\mathrm{Sr}$ content.

\subsubsection{RS study in $\mathrm{La}_{1-x} \mathrm{Sr}_{\mathbf{x}} \mathrm{MnO}_{3}$ films with different $\mathrm{Sr}$ content}

The analysis performed before was applied to the $x=0.3$ sample with the three different resistive states regions. The raw data of the $G(V)$ conductance curves for $x=0.3$ and $x=0.2$ films are shown in figure 3.25 ((a) and (b), respectively). A similar trend is found for both samples. The $G(V)$ curves of the HRS of the two films present an evident decrease of the $\mathrm{ZBC}$ whereas the shape of the curves is kept from the pristine states to the HRS.

a)

b)

FIGURE 3.25: Average conductance (G) cuves for pristine and high resistive states. (a) $x=0.3$ film. (b) $x=0.2$ film.

Then, the processed $Z B C$ values are extracted from the $\mathrm{dI} / \mathrm{dV}$ curves shown in 3.25. The comparison of the $\mathrm{ZBC}$ values obtained in this way for the pristine states of $x=\{0.3,0.2,0.15\}$ and the two HRS studied in the $x=\{0.3,0.2\}$ films are shown in figure 3.26 as filled dots. They have been plotted as a function of the $\mathrm{Mn}$ formal valency. The linear fit of the $\mathrm{ZBC}$ of the pristine states as a function of the Mn nominal valency presented in figure 3.22 is also plotted as a dashed line in the figure.

As also stated before, the HRS is induced upon the application of an electric field by oxygen excorporation from the structure accompanied by simultaneous decrease of 


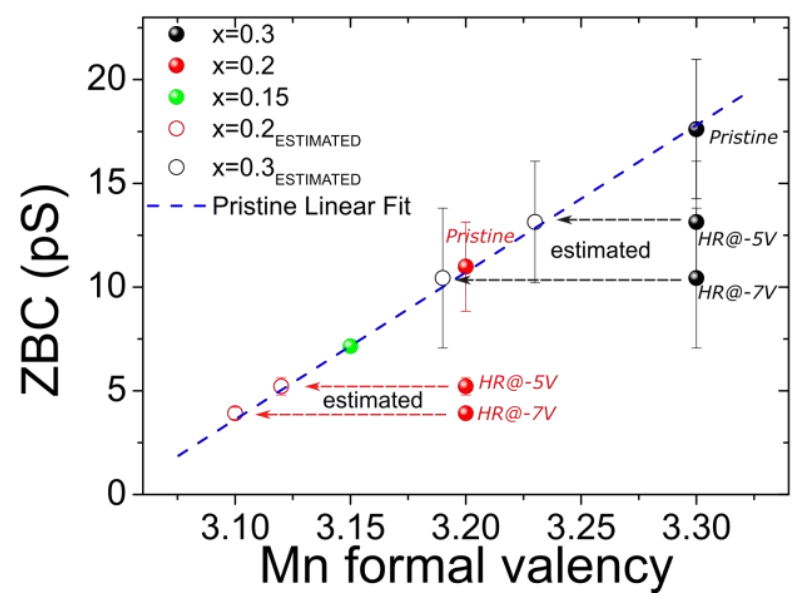

FIGURE 3.26: ZBC values as a function of the the Mn formal valency. Filled represent the raw data whereas open dots correspond to the estimated values once the ZBC values of the HRS for both films were projected into the linear fit of the $\mathrm{ZBC}$ values of the pristine state.

the free carriers in the system. The oxygen vacancies generated in this way within the structure produces a valence change of $\mathrm{Mn}$, and therefore, some $\mathrm{Mn}^{4+}$ is transformed into $\mathrm{Mn}^{3+}$. The simultaneous decrease of the number of carriers drives the system into a HRS linked to the metal-insulator transition. Up to now and by means of electrical characterization, no estimation of the change in the Mn valence state was achieved. However, STS has provided us with valuable data to achieve a rough assessment of the change in the Mn valence, and therefore, the amount of oxygen vacancies generated in the HRS of the system.

First, let us consider that the films are stoichiometric in the as-grown state. In these films, the Mn valence state is only determined by the concentration of metals (La and $\mathrm{Sr}$ ) introduced in the precursor solution. Other effects such as low dimensionality or strain will also not be taken into account in this first approach. Under these hypothesis, the stoichiometric formula of the compounds is was already shown in equation 3.1, where the formal (nominal) valence state of the $\mathrm{Mn}, \mathrm{Mn}_{\mathrm{FV}}$, was shown to be $M n_{\mathrm{FV}}=3.3,3.2,3.15$ for Sr contents of $x=\{0.3,0.2,0.15\}$,respectively. Then, if an intense electric field pointing downwards is applied at the nanoscale, oxygen excorporation is favoured and hence oxygen vacancies are created in the system. For the sake of charge neutrality, the chemical formula describing the LSMO compound in such a HRS is:

$$
\mathrm{La}_{1-x} \mathrm{Sr}_{x} \mathrm{Mn}_{\mathrm{FV}}^{\mathrm{HRS}} \mathrm{O}_{3-\delta}
$$

Where $\delta$ is the amount of excorporated oxygen, and $\mathrm{Mn}_{\mathrm{FV}}^{\mathrm{HRS}}$ has a new valence state directly related to $\delta$. As mentioned above, the filled dots in figure 3.26 represent the $\mathrm{ZBC}$ raw data for pristine and HRS states of the samples measured. The ZBC data obtained in the HRS is here represented with the same $\mathrm{Mn}_{\mathrm{FV}}$ as the pristine state, i.e., $\mathrm{Mn}_{F V}^{3.3}$ for $\mathrm{x}=0.3$ and $\mathrm{Mn}_{F V}^{3.2}$ for $\mathrm{x}=0.2$. In here, we propose that an estimation of the new Mn valence state in the HRS can be made by projecting the $\mathrm{ZBC}_{\mathrm{HRS}}$ on the

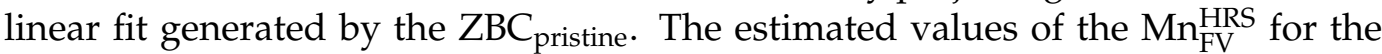
experimental ZBC are plotted as open circles of the correspondent colour (black for 
$\mathrm{x}=0.3$ and red for $\mathrm{x}=0.2$ ) close to the fitted line. In both cases, the changes in the $\mathrm{Mn}$ valence are estimated to be $\Delta \mathrm{Mn}=\mathrm{Mn}_{\mathrm{FV}}^{\text {pristine }}-\mathrm{Mn}_{\mathrm{FV}}^{\mathrm{HRS}} \approx 0.1$.

From the estimated values of the $\mathrm{Mn}_{\mathrm{FV}}^{\mathrm{HRS}}$ derived from this plot, further calculations might be perform. In particular, figure 3.27 (a) shows the relative change for the different resistive states regions in each film. Figure 3.27 (b) shows the total oxygen content of the film by applying charge neutrality principle in equation 3.2. Both plots reveal that approximately the same amount of oxygen is excorporated under the same applied electric field. Therefore, as a first approach we can conclude that, regardless the initial Sr content, we were able to generate a similar amount of oxygen vacancies in both samples, when the same bias is used to induce the HRS.

a)

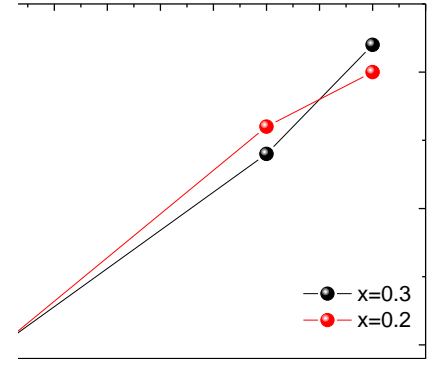

b)

FIGURE 3.27: Extrapolation showing the relative decrease in the Mn average valence state in both samples in the generated HRS. The inset show the calculated new oxygen content of the both films.

However, we have found differences in the decrease of the $\mathrm{ZBC}$ between both films in their respective resistance states. Figure 3.28 shows the decrease in the ZBC in percentage for the different resistive states regions in both samples. The values were calculated with respect to their pristine value. Both films shows the same trend, and the larger the $\mathrm{V}_{\text {Bias }}$ used to generate the HRS, the larger decrease in the ZBC. However, this decrease in the $Z B C$ is found to be larger in the $x=0.2$ than in the $x=0.3$ case. This late calculation is made directly from the raw data and no extrapolation or any other approximation was made.

FIGURE 3.28: Decrease of the ZBC as a function of the generated resistive state. Values were normalized to their pristine value.

Under our approximation, we showed that the same amount of oxygen vacancies were generated in both systems at a given resistive state, since a change of $\Delta \mathrm{Mn} \sim 0.1$ is found in the two cases. Therefore, the same proportion of $\mathrm{Mn}$ is 
transformed from $\mathrm{Mn}^{4+}$ to $\mathrm{Mn}^{3+}$. However, the initial ratio $\mathrm{Mn}^{4+} / \mathrm{Mn}^{3+}$ is different in both films, and therefore, the final ratio in the HRS will be accordingly different as well. This means that both systems present a different response in their electronic structure. The same amount of oxygen excorporation induces a deeper decrease in the number of states available at the Fermi level $(Z B C)$ in the $x=0.2$ than in the $x=0.3$. The specific composition of each film determined the relation $\mathrm{U} / \mathrm{W}$ and the charge carrier concentration of the systems. In the $\mathrm{x}=0.2$ case, a smaller variation in either the $\mathrm{U} / \mathrm{W}$ or the carrier concentration can induce an insulating state whereas the $x=0.3$ is in the metallic state. This situation is schematically illustrated in the figure 3.13. When the RS-MIT is induced, the closer proximity to the MIT line of the $\mathrm{x}=0.2$ film produces a larger decrease in the $\mathrm{ZBC}$ than in the $\mathrm{x}=0.3$ case. Thus, we have demonstrated via STS experiments that the resistive switching effect in these compounds is closely linked to the strong electron-electron correlation of these materials, and consequently, to their metal-insulator transition (MIT). The use of an intrinsic property such as the MIT for non-volatile memory applications could further boost the performance of future memristors, thus enhancing reproducibility, endurance, retention and the opportunity to generate multilevel resistive states.

\subsection{Electrical transport properties of HRS areas}

The study of the electrical transport properties of HRS areas by means of conventional 4-point measurements will be presented in this section. Our approach consists of inducing a HRS with the C-AFM on a LSMO bridge defined by optical lithography. Then, the resistivity of the bridge is evaluated as a function of temperature with a 4-point configuration. The study of the transport properties of the HRS allows the investigation of the modification of the MIT as a function of the voltage used to induce the HRS.

\subsubsection{Sample preparation and measurement protocol}

Experiments in this section were performed in a $10 \mathrm{~nm} \mathrm{La}{ }_{0.8} \mathrm{Sr}_{0.2} \mathrm{MnO}_{3}$ sample. First, a lithography process is performed to define a square grid of $100 \mu \mathrm{m} \times 100 \mu \mathrm{m}$ . Then, a $100 \mathrm{~nm}$ Au layer was deposited by DC sputtering followed by a lift-off process in an acetone ultrasonic bath for 20 seconds. Next, a second lithography process is carried out to define bridges of $30 \mu \mathrm{m}$ long by $15 \mu \mathrm{m}$ width. The bridges were finally defined by ion-milling in Ar atmosphere. In these bridges HRS at different bias will be induced by C-AFM and then resistivity measurements in a 4-point configuration will be performed. Either for C-AFM or resistivity measurements, the independent bridge is connected to the respective sample holders by ultrasonic wire bonding. Figure 3.29 shows optical micrographs of the film at the end of the preparation process (a) and a zoom of the bridge (b). Light red shows the region isolated defined by photolithography to avoid shortcuts with the overall LSMO layer. The electrodes employed in the resistivity measurements are indicated in the image in black as I+, V+, V- and I-. For C-AFM measurements, only I+ and I- were connected to the AFM sample holder. Au marks close to the bridge are used only as a guide references.

The following measurement protocol was established for these measurements: 
a)

b)

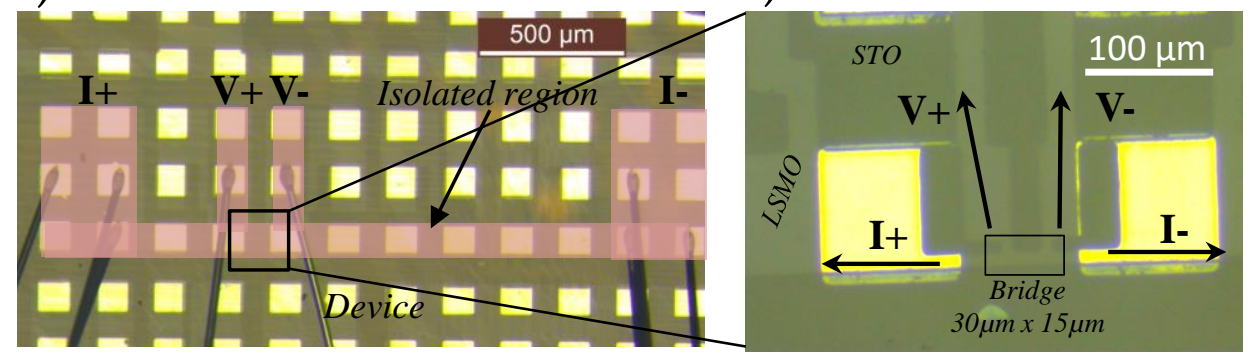

FIGURE 3.29: (a) Optical image of the film. Light red shows the isolated region (lithograpy + ion-milling etching). Electrodes for resistivity measurements are labelled in black. (b) Zoom of the bridge where measurements were performed (between $V+$ and $V$-).

1. First, we need to choose properly the voltages at which the bridge will be scanned by the tip. We need to assure that the full thickness of the film is transformed in the HRS in order to fully sense the electrical transport properties of the LSMO bridge in its high resistance state. In this way, we avoid to have a network of two effective resistances working in parallel and the intrinsic properties of the HRS will be fully measured. Therefore, rings at different voltages will be previously induced close to the bridge area to visualize the HRS cylindrical wall and the voltage required to reach the substrate as explained in section 3.1.3 with the observation of the rings.

2. The resistivity of the bridge will be measured. With the 4-point configuration shown in figure 3.29 only the bridge will be testedMeasurements are performed in a commercial PPMS (Quantum Design) from 400K down to $10 \mathrm{~K}$. The resistance state and the MIT will be firstly characterized for the pristine state.

3. The sample is taken out of the PPMS and mounted at the C-AFM holder. The AFM tip is located at the bridge. Then, a HRS is induced by scanning the bridge at the chosen $\mathrm{V}_{\text {bias }}$. After the nanostructuration, a resistance map at low bias is acquired to check the generated high resistance state of the bridge by C-AFM.

4. The sample is mounted again in the resistivity holder, and introduced in the PPMS chamber. A resistivity curve as a function of temperature is obtain for the bridge in the generated HRS.

5. Steps 3 and 4 can be repeated to generate a LRS by scanning the bridge with the C-AFM at a proper $\mathrm{V}_{\text {bias }}>0$ and then acquiring the resistivity curve in the PPMS.

\subsubsection{Selection of voltages to induce the HRS}

As explained above, to assure the electrical response of the HRS in the resistivity measurements, a volume switching of the full thickness of the film should be achieved. To infer the voltages required to do so, some HR rings at $-6 \mathrm{~V},-8 \mathrm{~V}$ and $-10 \mathrm{~V}$ were induced in the vicinity of the bridge. Figure 3.30 shows the topography and resistance maps after inducing the HR rings. 
a)

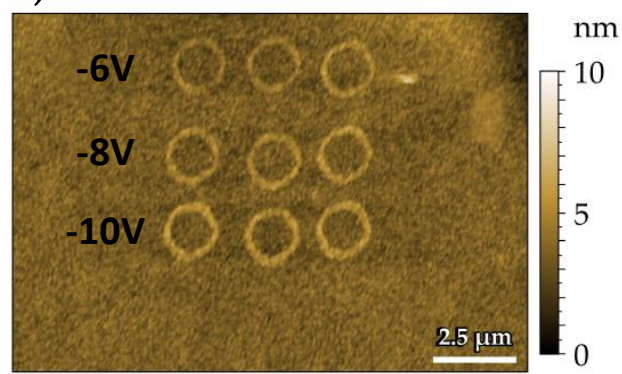

b)

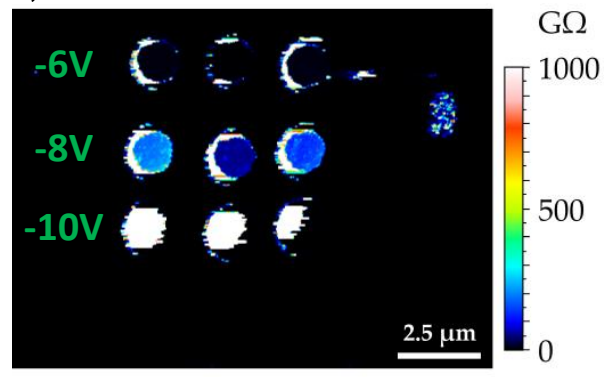

FIGURE 3.30: Topography (a) and resistance map (b) of the region were the HRS rings were generated. The voltage used in each set of rings is indicated in the figure.

For the 3 cases, a lift in the topography is found where the tip was scanning. This lift is below $1 \mathrm{~nm}$. This change could be ascribed to the presence of oxygen vacancies, which tends to enlarge the c-axis of the film but if not too high voltages are applied, this modification is a completely reversible phenomena. The response obtained in the resistance map strongly varies with applied voltage. Three different situations arise.

HRS induced at $-6 \mathrm{~V}$ : The pristine non-modified inner and outer parts of the ring are electrically connected. Volume switching of the full thickness of the film was not achieved. Therefore, larger $\mathrm{V}_{\text {Bias }}$ should be employed.

HRS induced at $-\mathbf{8 V}$ : In the resistance map, a certain blocking of the current is achieved although the full thickness has not been transformed. It suggests a thin layer close to the interface with the substrate that still might be electrically conductive. This voltage will be used in one of the brigdes of the sample.

HRS induced at -10V: A fully developed volume switching was achieved in this case. No current is measured in the inner non-modified regions of the rings. This voltage will also be used in the characterization of the HRS.

\subsubsection{The $-8 \mathrm{~V}$ HRS case}

In this case, a $-8 \mathrm{~V}$ scan was selected to induce a HRS in the bridge. First, a resistance map was acquired at low bias to characterize the pristine state of the bridge by CAFM. Figure 3.31 shows the topography and the resistance state of the brigde in its pristine state. I+, I-, V+ and V-connection bridges are also seen in both images.

Before any modification, in its pristine state, a resistivity curve as a function of temperature is acquired in the PPMS. For this device, a $\mathrm{T}_{\mathrm{MIT}}=343 \mathrm{~K}$ is found. A metallic state is well developed at room temperature with a $\rho=8.4 \mathrm{~m} \Omega \cdot \mathrm{cm}$

Once the $R(T)$ curve is performed, the sample is mounted in the C-AFM to transform the bridge into a HRS. Locating the tip in the bridge, a $-8 \mathrm{~V}$ scan is applied along the whole bridge. An schematic of the experiment is shown in figure 3.33. Once the HRS has been induced in the bridge, a resistance map at low bias is acquired to check the induction of the resistance state in the bridge. An homogeneous and dense HRS dominates the bridge. 
a)

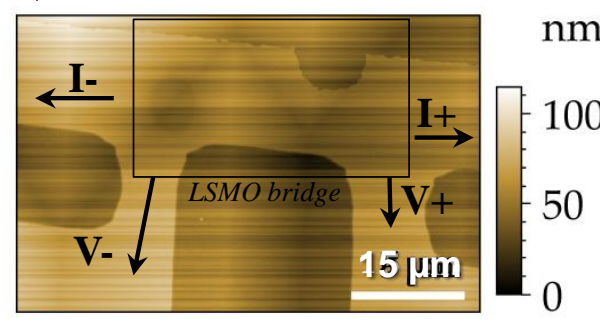

b)

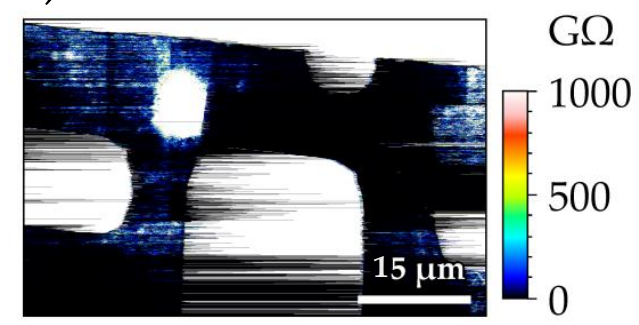

FIGURE 3.31: (a) AFM topography image of the bridge in the $10 \mathrm{~nm}$ $\mathrm{La}_{0.8} \mathrm{Sr}_{0.2} \mathrm{MnO}_{3}$ thin film. (b) Simultaneuosly acquired resistance at $1 \mathrm{~V}$.

FIGURE 3.32: Resistivity curve as a function of temperature for the bridge shown in figure 3.31 .

A second $R(T)$ is acquired to characterize the electrical response of the HRS. R(T) curves where successfully measured in the $400 \mathrm{~K}-10 \mathrm{~K}$ range and they are shown together in figure 3.34 (a). A clear increase in the resistance of around one order of magnitude is found in the whole temperature range for the HRS. For clarity, in the right picture, the raw resistance data have been normalized to its maximum value. For the HRS, values of $\mathrm{T}_{\mathrm{MIT}}^{\mathrm{HRS}}=325 \mathrm{~K}$ and a $\mathrm{R}_{\mathrm{RT}}^{\mathrm{HRS}} \approx 64 \mathrm{~m} \Omega \cdot \mathrm{cm}$ is found. Compared to its pristine state, the bridge in the HRS generated at $-8 \mathrm{~V}$ with the C-AFM presents a $\Delta \mathrm{T}_{\mathrm{MIT}}=\mathrm{T}_{\mathrm{MIT}}^{\text {Pristine }}-\mathrm{T}_{\mathrm{MIT}}^{\mathrm{HRS}}=18 \mathrm{~K}$ and a resistivity ratio $=\frac{\rho_{\mathrm{HRS}}}{\rho_{\text {Pristine }}} \approx 7.5$ at room temperature.

These shift in the $\mathrm{T}_{\mathrm{MIT}}$ and increase of the resistance were achieved by scanning the bridge under test at $-8 \mathrm{~V}$. With this bias (see figure 3.30) a completely full switching was not achieved although some blocking of the current was observed.

The shift in the $\mathrm{T}_{\mathrm{MIT}}$ and the increase of the resistance were achieved by scanning the bridge under test at $-8 \mathrm{~V}$. With this bias (see 3.30), a completely full switching through thickness was not achieved although some blocking of the current was observed. Thus we explained

\subsubsection{The -10V HRS case}

In this case, a $-10 \mathrm{~V}$ was used to induced the MIT by RS in the bridge. The used of $-10 \mathrm{~V}$ assures that the full thickness of the area under the tip will be transformed into a HRS in a volumetric manner. Left and center image in figure 3.35 show the 
a)

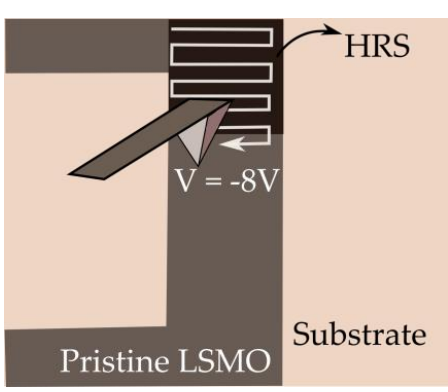

b)

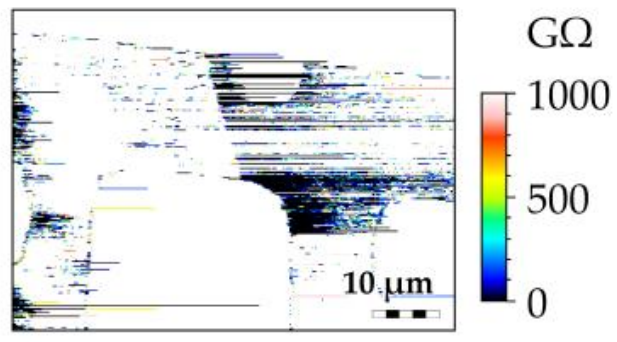

FIGURE 3.33: (a) Illustration of the experiment realization showing the tip scanning along the LSMO bridge at $-8 \mathrm{~V}$ and therefore inducing a HRS in it. (b) Resistance map acquired at $1 \mathrm{~V}$ of the bridge after inducing the HRS .

a)

b)

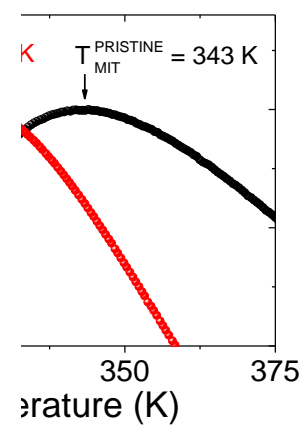

FIGURE 3.34: (a)Resistance of the bridge as a function of temperature in its pristine and HRS generated at $-8 \mathrm{~V}$. (b) Resistance of the bridge normalized to its maximum value. $\mathrm{T}_{\mathrm{MIT}}$ are indicated with arrows.

AFM topography map and the resistance map at $1 \mathrm{~V}$ of bridge in its pristine state. In this case, the device was $40 \mu \mathrm{m}$ long by $12 \mu$ width.

In this section a $-10 \mathrm{~V}$ was used to induced the MIT by RS in the bridge. According to the rings test, $V_{\text {bias }}=-10 \mathrm{~V}$ will assure that the full thickness of the area under the tip will be transformed into a HRS, in a volumetric manner. Figure 3.35 show the AFM topography map (a) and the resistance map of bridge in its pristine state at $1 \mathrm{~V}$ (b). In this case, the dimensions of the bridge was $40 \mu \mathrm{m}$ long by $12 \mu \mathrm{m}$ width.

Some regions with a higher resistance are seen in the pristine bridge (figure 3.35 (b)). However, when the RS is induced by a $-10 \mathrm{~V}$, a full HRS is developed in the whole bridge (figure 3.35 (c)).

As described in the protocol at the beginning of this section, prior to the induction of the HRS, a $\rho(T)$ curve was performed to characterize the pristine state of the bridge. Figure 3.36 (a) shows the raw data obtained for the resistivity of the bridge in its pristine and in the HRS induced at $-10 \mathrm{~V}$. At room temperature, the resistivity value in the pristine state and in the HRS generated at $-10 \mathrm{~V}$ are $\rho_{\text {prist }}=41 \mathrm{~m} \Omega \cdot \mathrm{cm}$ and $\rho_{\mathrm{HRS}}=4 E \cdot 10^{3} \mathrm{~m} \Omega \cdot \mathrm{cm}$

The resistivity of the bridge increases by two orders of magnitude at room temperature and leads $t$ for the HRS and the insulating state is maintained down to lower temperatures. For an easier comparison of the $\mathrm{T}_{\mathrm{MIT}}$, the normalized resistance (to 
a)

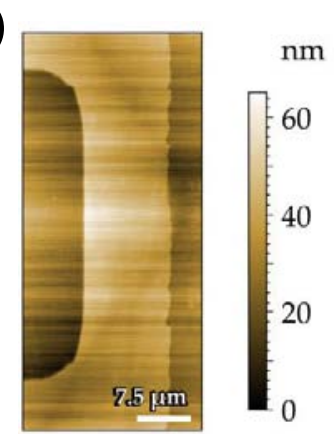

b)

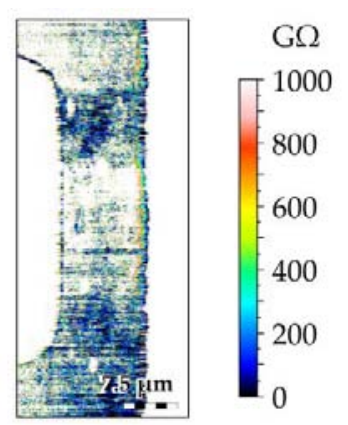

c)

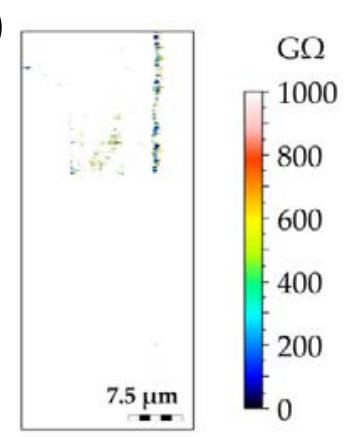

FIGURE 3.35: (a) AFM topography and (b )resistance map at $1 \mathrm{~V}$ of the bridge in its pristine state. (c) Resistance map at $1 \mathrm{~V}$ in the same bridge after the induction of the HRS at $-10 \mathrm{~V}$.

a)

b)

FIGURE 3.36: (a) Resistance of the bridge as a function of temperature in its pristine and HRS generated at $-10 \mathrm{~V}$. (b) Resistance of the bridge normalized to its maximum value. $\mathrm{T}_{\mathrm{MIT}}$ are indicated with arrows.

its maximum value). For the pristine state, a $\mathrm{T}_{\mathrm{MIT}}^{\text {Pristine }} \approx 316 \mathrm{~K}$ is found, while for the HRS is $\mathrm{T}_{\mathrm{MIT}}^{\mathrm{HRS}} \approx 199 \mathrm{~K}$. This means a shift of $\Delta \mathrm{T}_{\mathrm{MIT}}=117 \mathrm{~K}$.

The resistivity of the bridge increases by two orders of magnitude and leads to a ratio $\rho_{H R S} / \rho_{\text {prist }} \approx 100$ in the whole temperature range. For an easier comparison of the $\mathrm{T}_{\mathrm{MIT}}$, the normalized resistances (to their maximum value) are shown in figure 3.36 (b). For the pristine state, a $\mathrm{T}_{\mathrm{MIT}}^{\text {Pristine }} \approx 316 \mathrm{~K}$ is found, while for the HRS is $\mathrm{T}_{\mathrm{MIT}}^{\mathrm{HRS}} \approx 199 \mathrm{~K}$. This means a shift of $\Delta \mathrm{T}_{\mathrm{MIT}}=117 \mathrm{~K}$, much larger than the one observed in the previous experiment performed at $-8 \mathrm{~V}$.

We tried to resto the LRS in the switched bridge by scanning it at $\mathrm{V}_{\text {bias }}=+10 \mathrm{~V}$. To do so, the sample was mounted back into the C-AFM holder.

Figure 3.37 shows an schematic view of this last experiment (a) and the corresponding resistance map acquired after the process (b). This image should be compared to HRS generated at $-10 \mathrm{~V}$ shown in figure 3.35 (c). After inducing the LRS. a less resistive state has been generated, although it is not as conductive as in the pristine case (figure 3.35 (b)).

After the C-AFM modification, the sample was brought again to the PPMS. The resistivity of the bridge in the LRS as a function of temperature is then characterized. Results are shown in figure 3.38 (a). To facilitate the comparison, this last measurement is plotted together with the data obtained for the pristine and the HRS cases. 
a)

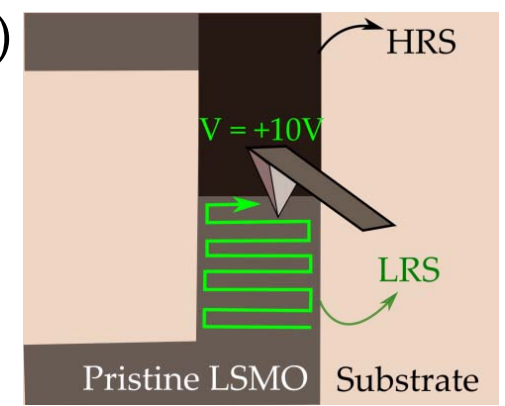

b)

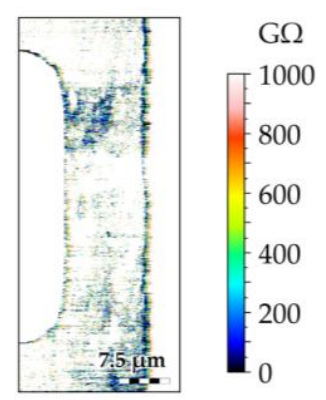

FIGURE 3.37: (a) Schematic representation of the C-AFM tip with $\mathrm{V}_{\text {bias }}=+10 \mathrm{~V}$ scanning along the bridge. This induces oxygen incorporation with generates a LRS. (b) Resistance map of the bridge at $1 \mathrm{~V}$ after generating the LRS.

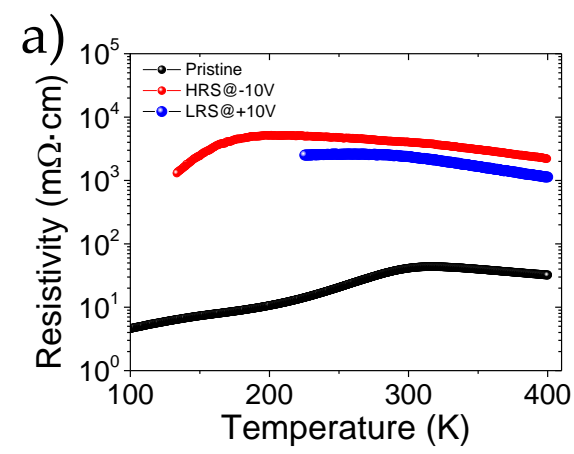

b)

FIGURE 3.38: (a) Resistance of the bridge as a function of temperature in its pristine, HRS generated at $-10 \mathrm{~V}$ and LRS generated after a $+10 \mathrm{~V}$ scan. (b) Resistances of the bridge normalized to its maximum value. $\mathrm{T}_{\mathrm{MIT}}$ are indicated with arrows.

Figure 3.38 (a) shows the raw data of the resistivity for the different resistance states. In blue the LRS is shown. A slightly less resistive state than for the HRS is found. Remarkably, the recovery of the MIT is much notable with a $\Delta \mathrm{T}_{\mathrm{MIT}}=\mathrm{T}_{\mathrm{MIT}}^{\mathrm{LRS}}-\mathrm{T}_{\mathrm{MIT}}^{\mathrm{HRS}}=63$. In figure $3.38(\mathrm{~b})$, the normalized resistance values are shown. Arrows indicate the $\mathrm{T}_{\mathrm{MIT}}$ for the different resistance states.

\subsubsection{Discussion}

From the results shown in this section, two main parameters have been extracted for discussion: The shift of the $\mathrm{T}_{\mathrm{MIT}}$ and the resistance ratio between states. As described above, two voltages, $-8 \mathrm{~V}$ and $-10 \mathrm{~V}$ were employed to generate different resistance states in the bridge.

For the $-\mathbf{8 V}$ case: A resistance ratio between the pristine and the HRS of almost 10 is achieved in the bridge in the whole temperature range. A shift in the transition temperature is observed, $\Delta \mathrm{T}_{\mathrm{MIT}}=343-325 \mathrm{~K}=18 \mathrm{~K}$.

For the $\mathbf{- 1 0 V}$ case: The resistance ratio found in the bridge between the pristine and the HRS is close to 100 in the full temperature range. The shift in the transition temperature observed is $\Delta \mathrm{T}_{\mathrm{MIT}}=316-199 \mathrm{~K}=117 \mathrm{~K}$. Furthermore, a partial LRS was induced in the bridge by a $+10 \mathrm{~V}$ scan. Resistance 
slightly decreases (Ratio LRS/Pristine $=57)$ while the $\mathrm{T}_{\mathrm{MIT}}^{L R S}=262 \mathrm{~K}$ giving a $\Delta \mathrm{T}_{\mathrm{MIT}}^{\text {Pristine-LRS }}=54 \mathrm{~K}$

These numbers are resumed in figure 3.39. Left image shows the resistance ratio between the modified resistance states (HRS and LRS, if any) and the pristine state. Right image represents the $\mathrm{T}_{\mathrm{MIT}}$ for both bridges in the measured resistance states in the bridge (pristine, HRS and LRS).

a)

b)

FIGURE 3.39: (a) Resistance ratio at room temperature as a function of the generated resistive state. (b) $\mathrm{T}_{\mathrm{MIT}}$ extracted from the $\mathrm{R}(\mathrm{T})$ curves for each resistive state.

Since the $\mathrm{T}_{\mathrm{MIT}}^{-8 \mathrm{~V}}$ is found to be $325 \mathrm{~K}$, the MIT was not fully surpassed in the bridge and therefore a small increase of the resistance happes. In contrast, in the $-10 \mathrm{~V}$ case, a bigger increase (ratio $\sim 100$ ) is found. In this case, the $\mathrm{T}_{\mathrm{MIT}}^{\mathrm{HRS}}-10 \mathrm{~V}=199 \mathrm{~K}$, well below RT. This means that the insulating state is kept down to lower temperatures, and specifically, at room temperature the MIT was fully crossed. The attempt to recover the original state in the bridge by a $+10 \mathrm{~V}$ scan generates a partially restored LRS, where $\mathrm{T}_{\mathrm{MIT}}^{\mathrm{LRS}}=262 \mathrm{~K}$. Therefore, at room temperature, the LRS has not been fully restored and consequently, only a small decrease of the resistance of the bridge is achieved. If the MIT would have been crossed back, a larger resistance change would be expected. In fact, in section 3.5, it will be shown for a proof-of-principle 3Terminal configuration that the initial resistance of pristine state could be recovered at a micrometric scale in a device-like geometry with microscopic W-Au tips.

\subsection{Atmosphere dependence of the RS effect}

Along this chapter, a full analysis of the RS mechanism in LSMO thin films has been performed. However, a more detailed microscopic view of the oxygen exchange mechanism inducing the MIT in LSMO thin films is required for a deeper understanding of the effect. To do so, I-V sweeps in direct contact with the bare surface of a $10 \mathrm{~nm} \mathrm{La}{ }_{0.8} \mathrm{Sr}_{0.2} \mathrm{MnO}_{3}$ in different atmospheres will be presented in this section. A closer look to the microscopic mechanism of the oxygen exchange with ambient, promoted upon the application of an electric field, will be presented.

However, to fully demonstrate the usability of LSMO layers as memristor devices, it is mandatory to have an encapsulated device with integrated electrodes. Therefore, we must avoid the exchange with ambient as mechanism. In order to overcome this problem, we propose the introduction of a thin layer on top of the LSMO active layer that acts as oxygen reservoir enabling direct ion exchange with the LSMO layer. 
Investigations of the RS performance in a variety of oxides at different atmospheres and moisture conditions have been widely studied in the literature [26, 29, 244, 270274]. In all the cases, a strong influence of the ambient gases on the RS properties by surface adsorption and chemical oxidation was found. Depending on the origin of the RS in each material, RS performance might be either enhanced [29] or diminished [272] in vacuum. In this section, we report the impact of the atmosphere on the RS properties of our LSMO thin films and bilayer $\mathrm{CeO}_{2} / \mathrm{LSMO}$ systems.

The experiments were performed in collaboration with Prof. Regina Dittmann and Mr. Benedikt Arndt at the Forschungszentrum Jülich (Jülich, Germany) during a two weeks short internship. Measurements were carried out in a home-made probe station system mounted inside a sealed chamber connected to a rotary vane pump. Vacuum conditions down to $P=10^{-5} \mathrm{mbar}$ can be achieved in this system. Besides, gas inlets allow to perform measurements at different gas partial pressures. In particular, pure $\mathrm{O}_{2}$ atmosphere at different pressures was also used in the experiments.

First, let us introduce the basic phenomenology of the RS effect in LSMO thin films studied by means of W-Au macroscopic tips.

\section{RS studies with microscopic tips}

As it was mentioned at the beginning of this chapter, another $\mathrm{PhD}$ thesis regarding the RS properties of LSMO thin films was being carried out in the group in parallel to this thesis by Dr. Rafael Ortega-Hernandez.

In his work, the I-V characteristics of the LSMO films were investigated by applying systematic voltage sweep loops $(0 \rightarrow+X V \rightarrow-X V \rightarrow 0)$ using a Keithley 4200 Semiconductor Characterization System and an Agilent 4156C Semiconductor Parameter Analyzer in a top-top configuration. All the measurements were performed in air at room temperature using $\mathrm{W}$-Au probes to contact the microdevices. A bipolar resistive switching behaviour was demonstrated at the micrometric scale only when measurement probes were located directly on top of the $\mathrm{La}_{1-\mathrm{x}} \mathrm{Sr}_{x} \mathrm{MnO}_{3}$ layer. No remarkable resistive switching could be induced when probes were located in metal electrodes (see figure 3.40). The observed I-V curves are fully consistent with the microscopic view of the RS mechanism stated along this thesis, derived from the C-AFM experiments.

In addition, Dr. Rafael Ortega-Hernandez investigated the RS characteristics of $\mathrm{ME} / \mathrm{CeO}_{2-x} / \mathrm{LSMO} / \mathrm{CeO}_{2-x} / \mathrm{ME}$ structures $(\mathrm{ME}=\mathrm{Ag}, \mathrm{Au}, \mathrm{Pt})$ as shown in figure $3.41(b)$.

First, he showed that $10 \mathrm{~nm}$ ALD- $\mathrm{CeO}_{2-x}$ grown on a conductive substrate 0 : $05 \%$ wt $\mathrm{Nb}: S r T i O 3$ does not show any RS characteristics in our equipment setup under $\pm 10 \mathrm{~V}$ sweeps. Thus, the formation of conducting filaments formed inside the $\mathrm{CeO}_{2-x}$ in such a MIM structure is ruled out. Then the bilayer $\mathrm{ME} / \mathrm{CeO}_{2-x} / \mathrm{La}_{0.8} \mathrm{Sr}_{0.2} \mathrm{MnO}_{3} / \mathrm{CeO}_{2-x} / \mathrm{ME}$ was evaluated. Although RS was observed for three different metal electrodes, special emphasis will be made on the Ag metal electrodes due to its better performance. A initialization process (figure 3.41 (a)) switches the device from a high insulating state to a less resistive state without reaching the full LRS of the device. Then (figure 3.41 (b)), negative voltage 
a)

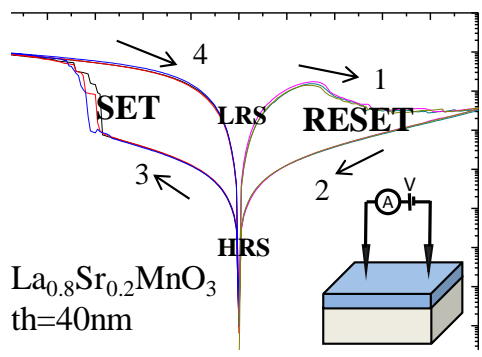

b)

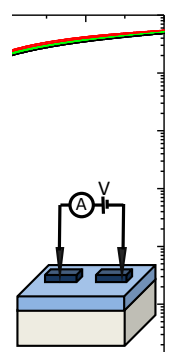

FIGURE 3.40: I-V curves performed with the W-Au micrometric tips in direct contact with the bare surface of the LSMO film (a) and on top of different metal electrodes (b). RS could only be induced when the tips were in direct contact with the film. Notice that RESET and SET processes in (a) occurs at the opposite bias polarity that in the

C-AFM case. However, the electric field polarity is the same.
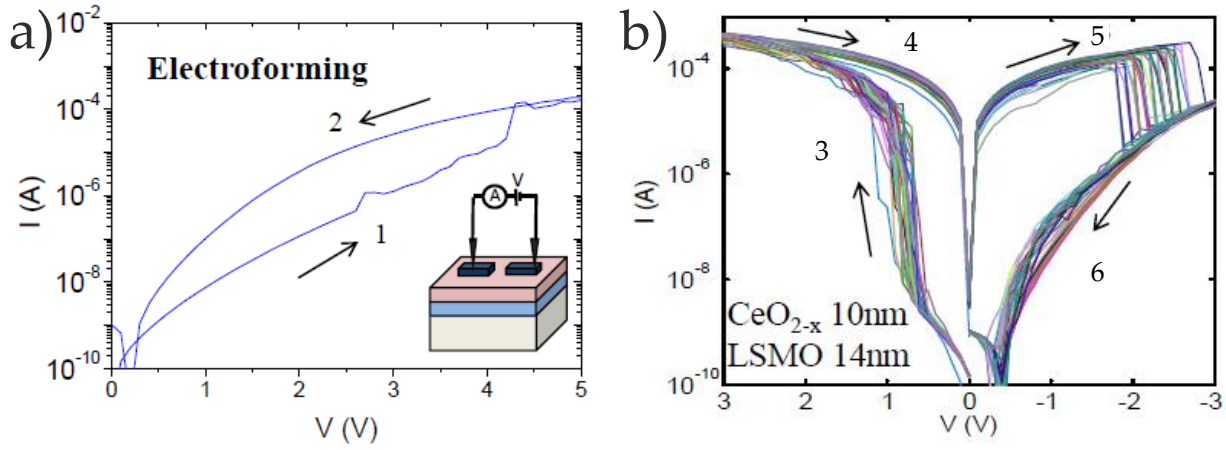

FIGURE 3.41: I-V curves measured in on $\mathrm{Ag} / \mathrm{CeO}_{2-\mathrm{x}} / \mathrm{La}_{0.8} \mathrm{Sr}_{0.2} \mathrm{MnO}_{3} / \mathrm{CeO}_{2-\mathrm{x}} / \mathrm{Ag}$ devices. (a) Initialization process and (b) subsequent bipolar RS cycles induced when probes are placed on metal electrodes for at least 100 cycles.

sweep induces a sharp increase of current where a change from HRS to LRS is observed (branch 1). The device remains in the LRS during the voltage back excursion (branch 2). At positive bias sweep, the LRS is preserved until a sharp decrease of the current leads to a change to the HRS (branch 3) which is retained in the voltage back sweep (branch 4 ).

Compared to the bare $\mathrm{La}_{0.8} \mathrm{Sr}_{0.2} \mathrm{MnO}_{3}$ layer, a remarkable decrease in the operation voltages are achieved. In addition, the resistive transitions present very sharp features. The resistance of both states is shown in figure 3.42 (a). A ratio of more than $10^{4}$ is found for at least 100 cycles for a $V_{\text {read }}=-0.5 \mathrm{~V}$. This represents an increase of more than one order of magnitude with respect to the bare LSMO film using micrometric probes.

For $\mathrm{CeO}_{2-x} / \mathrm{La}_{1-x} \mathrm{Sr}_{x} \mathrm{MnO}_{3}$ bilayer systems, the BRS is explained using the same oxygen ion exchange mechanism (figure 3.42). When a downwards-pointing electric field is applied,oxygen anions are attracted to the $\mathrm{CeO}_{2-x} / \mathrm{La}_{1-x} \mathrm{Sr}_{x} \mathrm{MnO}_{3}$ interface where they are trapped, inducing the transition from LRS to HRS (Reset) of the device. If an opposite electric field is then applied to the same electrode, the oxygen anions are released from the $\mathrm{CeO}_{2-x}$ layer and are driven back to the $\mathrm{La}_{1-x} \mathrm{Sr}_{x} \mathrm{MnO}_{3}$ switching the active layer to the initial LRS (Set). 
a)

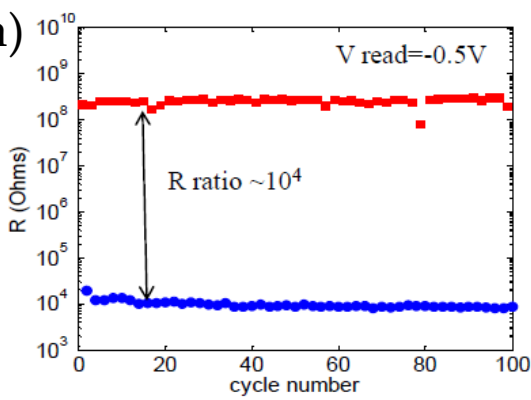

b)

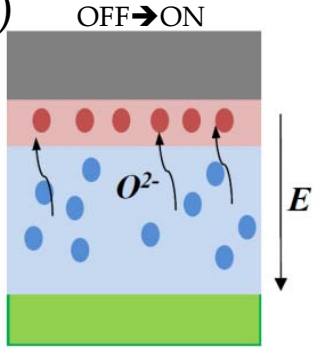

$\mathrm{ON} \rightarrow \mathrm{OFF}$

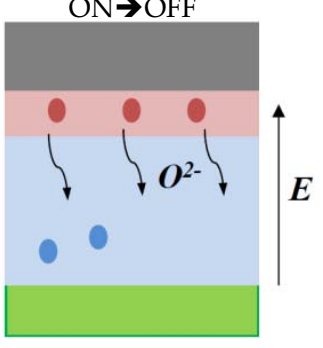

FIGURE 3.42: (

a) Resistance ratio of the 100 cycles shown in figure ?? in the $\mathrm{Ag} / \mathrm{CeO}_{2-\mathrm{x}} / \mathrm{La}_{0.8} \mathrm{Sr}_{0.2} \mathrm{MnO}_{3} / \mathrm{CeO}_{2-\mathrm{x}} / \mathrm{Ag}$ structure. (b) Schematic representation of the oxygen exchange process between the ceria layer and the active LSMO film.

As described above, this approach is followed in this section to perform I-V sweeps at different total and $\mathrm{O}_{2}$ partial partial pressures. Figure ?? shows a picture of the actual equipment and a schematic diagram of it. The same electric field configuration than in our AFM setup was used.

Measurements were carried out in a $10 \mathrm{~nm} \mathrm{La}{ }_{0.8} \mathrm{Sr}_{0.2} \mathrm{MnO}_{3}$ film grown onto STO and on a $50 \mathrm{~nm} \mathrm{Ag} / 10 \mathrm{~nm} \mathrm{CeO}{ }_{2-x} / 10 \mathrm{~nm} \mathrm{La}_{0.8} \mathrm{Sr}_{0.2} \mathrm{MnO}_{3}$. CSD-LSMO and ALD$\mathrm{CeO}_{2-x}$ thin films were deposited as described in chapter 2. For the Ag deposition, first an optical lithography process was performed to define a $100 \mu m x 100 \mu m$ square grid. Then, $50 \mathrm{~nm}$ of Ag were sputtered followed by a lift-off process in an ultrasonic bath of acetone. An image of the film is shown in figure 3.43.

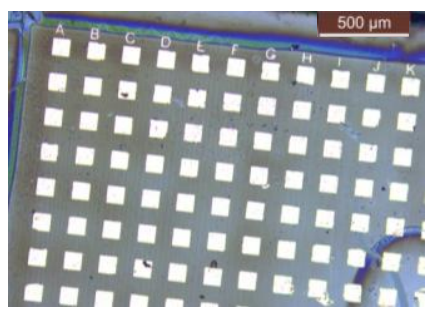

FIGURE 3.43: Optical micrograph of the $\mathrm{Ag} / \mathrm{CeO}_{2} / \mathrm{LSMO}$ strucures.

\subsubsection{RS at different total pressures}

I-V sweeps with the tips in direct contact with the bare LSMO film at ambient conditions were performed in a top-top configuration (figure 3.44 (a)). The protocol applied was $0 \rightarrow-10 \mathrm{~V} \rightarrow+10 \mathrm{~V} \rightarrow 0$. Towards negative voltages, a LRS $\rightarrow$ HRS transition is induced (1). Then, towards positive bias, the LRS is restored by the crossing the HRS $\rightarrow$ LRS transition (3). Repeatability cycles were performed up to $100 \mathrm{I}-\mathrm{V}$ curves at the same location. In the $\mathrm{Ag} / \mathrm{CeO}_{2-x} / \mathrm{La}_{0.8} \mathrm{Sr}_{0.2} \mathrm{MnO}_{3}$ case, the $\mathrm{I}-\mathrm{V}$ sweeps were performed between $\pm 2.5-3.5$ (3.44 (b)). First, an initialization process as the one shown in figure 3.41 is performed. A positive voltage sweep generates a sharp increase of the current inducing the HRS $\rightarrow$ LRS transition (1). The LRS is kept during the voltage excursion back (2). At negative bias, the LRS state is preserved until a sharp decrease in the current is found and the LRS $\rightarrow$ HRS occurs (3). The HRS is held in the excursion back (4). 
a)

c) b)

d)

FIGURE 3.44: Comparison of $\mathrm{I}-\mathrm{V}$ curves acquired in air and at low pressures. (a) The tips are in direct contact with the $\mathrm{La}_{0.8} \mathrm{Sr}_{0.2} \mathrm{MnO}_{3}$. (b) The tips are on top of the Ag electrodes in $\mathrm{Ag} / \mathrm{CeO}_{2} / \mathrm{La}_{0.8} \mathrm{Sr}_{0.2} \mathrm{MnO}_{3}$ structures. (c) Cumulative probability plots for the bare LSMO derived from (a). (d) Cumulative probability plots for the $\mathrm{Ag} / \mathrm{CeO}_{2} / \mathrm{LSMO}$ bilayer derived from (b).

Afterwards, the chamber was pumped overnight to achieve a base pressure of $P=$ $10^{-5}$ mbar. The same measurement protocol was repeated in both samples. Figure 3.45 shows The $\mathrm{I}-\mathrm{V}$ endurance cycles acquired at $P=10^{-5} \mathrm{mbar}$ and in air are shown in figure 3.44 (a) for the bare LSMO case and (b) for $\mathrm{Ag} / \mathrm{CeO}_{2} / \mathrm{LSMO}$ one.

In the bare LSMO case, the generated HRS at ambient conditions presents 1000 times higher resistance than the generated in vacuum conditions. The resistance value of the LRS and the HRS were evaluated at $\mathrm{V}_{\text {read }}=0.5 \mathrm{~V}$ and the cumulative probability was calculated and plotted in figure 3.44 (c). The lower LRS found in vacuum ( $R=10^{5} \Omega$ in ambient conditions and $R=10^{4} \Omega$ in vacuum), can be attributed to better contact between the tip and the sample at low pressures due to the decrease of the moisture level (the role of moisture is discussed later). In contrast, the base pressure change has a deep impact on the HRS. While in ambient conditions a large resistance ratio of $10^{2}-10^{3}$ is found, the ratio observed in vacuum is extremely diminished to a value of $\sim 2$. Therefore, we demonstrate that the RS is produced through an exchange with ambient and it has strong impact on the generated HRS.

In the $\mathrm{CeO}_{2} / \mathrm{LSMO}$ bilayer case, the general shape of the I-V curves is kept in both atmospheres. However, there are small changes between them observed. The resistance value of the LRS and the HRS were evaluated at $\mathrm{V}_{\text {bias }}=0.2 \mathrm{~V}$ and the cumulative probability was calculated and plotted in figure 3.44 (d). Compared to the response in air, an overall increase of the resistance at low pressures in both the LRS and the HRS is found. This decrease in the conductivity could be related 
to the influence of the absence of moisture. It has been reported that ceria basedmaterials has the capability of incorporating hydroxyl ions into their surface and into the bulk by interaction with oxygen vacancies $[275,276]$, forming protonic defects. These defects can diffuse through a Grotthuss mechanism, which is based on the fast rotational diffusion of the protonic defects and the proton transfer to the neighbouring oxygen ions [277], which is considered the rate determining step (rds). However, more experiments should be performed to fully addressed this issue.

Remarkably, the external atmosphere (and consequently, the aforementioned overall increase of the resistance) has no effect on the switching capabilities. A large resistance ratio of $10^{4}$ between the LRS and the HRS is found at air and at low pressures. This experiment confirms our hypothesis that the ion exchange occurs between the $\mathrm{CeO}_{2}$ and the LSMO layer and not through the interface with ambient. The encapsulated oxygen exchange might account also for the sharper resistive transitions observed in this case, in contrast to the LSMO film, where a smooth and progressive $\mathrm{ON} \rightarrow \mathrm{OFF}$ transition is found. This fact will be discussed in more detailed at the end of this section. Further studies should carefully address the variability of threshold voltages and retention measurements, which could help in the understanding of the switching process.

\subsubsection{RS at different oxygen pressures}

For the bare LSMO, starting from a low total base pressure achieved overnight $\left(P=10^{-5}\right.$ mbar $), \mathrm{O}_{2}$ gas is introduced in the chambers. By controlling the gas flow and the rotatory speed of the pump, a wide range of partial (and in this case, also total) $\mathrm{P}_{\mathrm{O}_{2}}$ was accessible. In particular, three different pressures were used, namely $0.74,0.22$ and 0.019 mbar. For stabilization purposes, measurements were performed at least one hour after the device showed the desired pressure set point. The same sweeping protocol as in the previous measurements was applied. Figure 3.45 shows the several I-V endurance cycles acquired for each pressure conditions.

a)

b)

FIGURE 3.45: (a) I-V curves with the tip in direct contact with the $\mathrm{La}_{0.8} \mathrm{Sr}_{0.2} \mathrm{MnO}_{3}$ acquired in pure $\mathrm{O}_{2}$ atmosphere at $0.75,0.22$ and 0.019 mbar . (b) Resistance of the LRS and the HRS at $\mathrm{V}_{\text {read }}=0.5 \mathrm{~V}$ extracted from the I-V cycles in (a).

I-V sweeps shows a large reproducibility at every $\mathrm{P}_{\mathrm{O}_{2}}$ tested. The resistance cumulative probability plots were extracted from these I-V cycles for the LRS and the HRS at $\mathrm{V}_{\text {bias }}=0.5 \mathrm{~V}$ (figure $3.45(\mathrm{~b})$ ). In the figure, it can be observed that, while 
the $R_{\mathrm{LRS}}$ has no dependence on the external pressure, the generated $\mathrm{R}_{\mathrm{HRS}}$ decreases when decreasing $\mathrm{P}_{\mathrm{O}_{2}}$ in the chamber.

Figure 3.46 shows the average resistance value for the LRS and HRS as a function of the partial $\mathrm{p}_{\mathrm{O}_{2}}$ in the chamber. As observed in the figure, the resistance of the LRS does not depend on the external $\mathrm{P}_{\mathrm{O}_{2}}$. Its value is $\mathrm{R}_{\mathrm{LRS}}=30-50 \mathrm{k} \Omega$. However, a change of three orders of magnitude is found when $\mathrm{p}_{\mathrm{O}_{2}}$ varies from ambient conditions, where the oxygen partial pressure is $2 \cdot 10^{2}$ mbar, to high vacuum conditions $\left(10^{-6}\right)$. No significant differences were observed in the voltages at which the transitions occurs.

FIGURE 3.46: Average resistance values for the LRS and the HRS as a function of the $\mathrm{P}_{\mathrm{O}_{2}}$ in the chamber.

These experiments already suggest us a picture in which the oxygen exchange through the atmosphere drives the system into the HRS. However, it has been reported that moisture might strongly affect [277] or even suppress [26] the resistive switching effect in some devices. In this sense, let us first examine in more detail the influence of moisture in the switching behaviour in our films before discussing the mechanism.

\subsubsection{RS at different moisture conditions}

In chapter 2, we discussed the influence of moisture in the C-AFM experiments. There, we showed that the relative humidity should be decreased below $5-10 \%$ to obtain reproducible and stable measurements. In addition, we showed that irreversible topography damage could be inflicted to the film under high bias. Therefore, this suggests that moisture is not the driving force behind the RS exchange, but its presence can be detrimental to the RS performance of the films.

In the case of the microscopic W-Au tips, a similar performance to the nanoscale RS effect is obtained at a larger microscopic case in air. To further approach the influence of moisture on the switching capabilities, the RS characteristics of the bare LSMO layer were tested in variable relative humidity environment in a pure $\mathrm{N}_{2}$ atmosphere. These preliminary tests were performed by Mr. Christoph Baumer fruit of the collaboration with the Forschungszentrum Jülich. Specifically, the I-V sweeps were performed at two moisture conditions, close to the values found in our measurements in air, where large resistance ratios of $10^{2}-10^{4}$ are found: $25 \%$ and $50 \% \mathrm{RH}$.

The I-V cycles for $25 \%$ and $50 \%$ relative humidity (figure 3.47 (a)) show reproducible I-V curves for at least 50 cycles with the same qualitative shape described 
a)

b)

FIGURE 3.47: (a) Resistance ratio at room temperature as a function of the generated resistive state. (b) $\mathrm{T}_{\mathrm{MIT}}$ extracted from the $\mathrm{R}(\mathrm{T})$ curves for each resistive state.

before. Similarly, the resistance values at $\mathrm{V}_{\text {bias }}=0.5 \mathrm{~V}$ were extracted to calculate the resistance cumulative probability function (figure3.47 (b)). The LRS presents slightly lower resistance value in $25 \%$ water atmosphere. This justifies the lower LRS found in vacuum. For both relative humidity values similar HRS are generated leading to resistance ratios of $5-10$. The similar I-V sweeps obtained at both humidity levels suggests that moisture does not have a key role in the origin of the RS effect of this compounds. Rather, the similar and small resistance ratios obtained might be originated from a low partial air pressure (and consequently, low oxygen partial pressure) arising from gas impurities. However, a deeper evaluation should be performed to further elucidate the possible role of electrochemically induced water-splitting reaction [26].

\subsubsection{Discussion}

Understanding oxygen incorporation and excorporation reactions in oxide materials $[278,279]$ is still a relevant topic in fundamental research and a keypoint on the functionalization of electroceramic devices, such as sensors and solar oxides fuel cell (SOFC) cathodes [280, 281]. Despite being one of the "simplest" examples of solid-gas chemical reaction, oxygen exchange is a very intricate process that is summarized in four steps [278]: Adsorption (from $\mathrm{O}_{2}$ gas to the surface of the solid), dissociation $\left(\mathrm{O}_{2}\right.$ molecule is splitted at the surface $)$, Surface migration $\left(\mathrm{O}^{-}\right.$or $\mathrm{O}^{2-}$ ions diffuse at the surface to find oxygen vacancies) and finally, incorporation $\left(\mathrm{O}^{2-}\right.$ ions incorporate into the lattice by recombining with an oxygen vacancy). On the other hand, the excorporation of oxygen from the lattice occurs due to an electrochemical reaction between the adsorbate species and an anion at the crystal interface, thus creating an oxygen vacancy in the lattice and forming an $\mathrm{O}_{2}$ molecule at the surface. These two processes are intrinsically asymmetric. However, in our case, the three first steps are common for both the oxygen excorporation and incorporation processes and are crucial to understand the impact of varying the oxygen partial pressure, $\mathrm{P}_{\mathrm{O}_{2}}$. Therefore, these steps will be described first properly before introducing the electric field-induced excorporation and incorporation of oxygen.

Adsorption: $\mathrm{O}_{2}$ gas molecules can get physisorbed and/or chemisorbed to the surface. There is no charge transfer between the adsorbated $\mathrm{O}_{2}$ and the surface in the case of the physisorption process. The energy of this adsorbate is very small $\left(\mathrm{E}_{\mathrm{Ads}}=-0.25 \mathrm{eV}\right)$ and hence the probability that this adsorbate 
is formed is very low [282]. In contrast, a chemisorption process involves charge transfer, thus presents a higher energy $\left(\mathrm{E}_{\mathrm{Ads}} \sim-2 \mathrm{eV}\right)$ and therefore $\mathrm{O}_{2}^{-}$adsorbate is more likely to be formed [282]. The reaction of the adsorption process is then:

$$
\mathrm{O}_{2}(g)+S_{\mathrm{ads}}+e^{-} \longrightarrow \mathrm{O}_{2}^{-} \cdot S_{\mathrm{ads}}
$$

$\mathrm{S}_{\mathrm{ads}}$ stands for site of adsorption. Oxygen vacancies and other defects can act as an adsorption site. However, the concentration of them is low and adsorption processes can occur in clean surfaces as well [281]. The formation of charged adsorbates $\left(\mathrm{O}_{2}^{-}, \mathrm{O}_{2}^{2-}, \mathrm{O}^{-}\right)$and their mutual electrostatic interaction (which produces deviations from Langmuir's isotherm) will lead to a certain coverage degree for each species $\Theta_{O_{n}^{j-}}$ that can be described in first approach as [283]

$$
\Theta_{O_{n}^{j-}}=K_{a d s}\left(p O_{2}\right)^{n / 2} \exp -\frac{j e_{0} \Theta \chi_{\max }}{k T}
$$

where $j$ is the adsorbate charge, $n$ the number of oxygen atoms it contains and $K_{a d s}=\exp -\Delta G_{a d s}^{0} / R T$ the mass action constant of adsorption $\chi_{\max }$ is the surface potential drop built up in case of full coverage $\Theta=1$. As seen in ref. [283], the surface coverage can change several orders of magnitude upon changes in the $p\left(O_{2}\right)$, thus playing a key role to understand the RS effect in LSMO thin films. However, the exact calculation of the coverage is not trivial, since different crystalline surfaces usually lead to modified coverage degree (see, for example [281]) and also might be sample dependent for their different steps or grain boundaries densities. Calculations [281] show that atomic adsorbates $\left(\mathrm{O}^{-}\right)$dominates the coverage with a larger presence than the molecular adsorbates $\left(\mathrm{O}_{2}^{-}, \mathrm{O}^{2-}\right)$. For transition metal oxides, it is estimated that there is around $10^{-2}-10^{-3}$ adsorbates per unit cell at $1 \mathrm{bar}$.

Molecular dissociation: Molecular dissociation of the absorbate $\mathrm{O}_{2}^{-}$is produced following the equation

$$
\mathrm{O}_{2_{\text {ads }}^{-}} \longrightarrow 2\left(\mathrm{O}^{-}\right)_{\text {ads }}
$$

$\mathrm{O}_{2 \text { ads }}^{-}$has a large $E_{\text {ads }}=-2.2 \mathrm{eV}$ that allows dissociation of the $\mathrm{O}_{2}$ molecule. The atomic surface coverage, also governed by Langmuir's equation is expected to be much larger than the molecular coverage. At reduced $\mathrm{p}\left(\mathrm{O}_{2}\right), \Theta$ is expected to decrease by a factor o $10-10^{2}$. Overall, the reaction of adsorption, charge transfer and molecular dissociation can be schematically written as

$$
\mathrm{O}_{2_{\mathrm{ads}}}+4 e^{-} \longrightarrow 2\left(\mathrm{O}^{-}\right) \text {ads }
$$

$\mathrm{O}_{\text {ads }}^{-}$has two negatives charges, one has been given to the system. Usually, the neither the adsorption nor the dissociation steps are not considered to be the rate determining step (rds) of the reaction, but might be relevant in the full incorporation/excorporation process.

Surface oxygen migration: Once the atomic species are adsorbated at the surface, either $\mathrm{O}^{-}$species or oxygen vacancies inside the system need to diffuse to be incorporated into the system to equilibrate the external $\mathrm{p}\left(\mathrm{O}_{2}\right)$ at a given T. In the excorporation process, an absorbated specie will be created and will need also to diffuse a certain distance to react with another adsorbate. This distance is called surface diffusion length and it depends strongly on the adsorbate 
coverage and, in turn, in the external $\mathrm{p}\left(\mathrm{O}_{2}\right)$. In LSMO, this step is considered the RDS of the process [279].

A schematic view of the three first steps of the process is shown in figure 3.48.

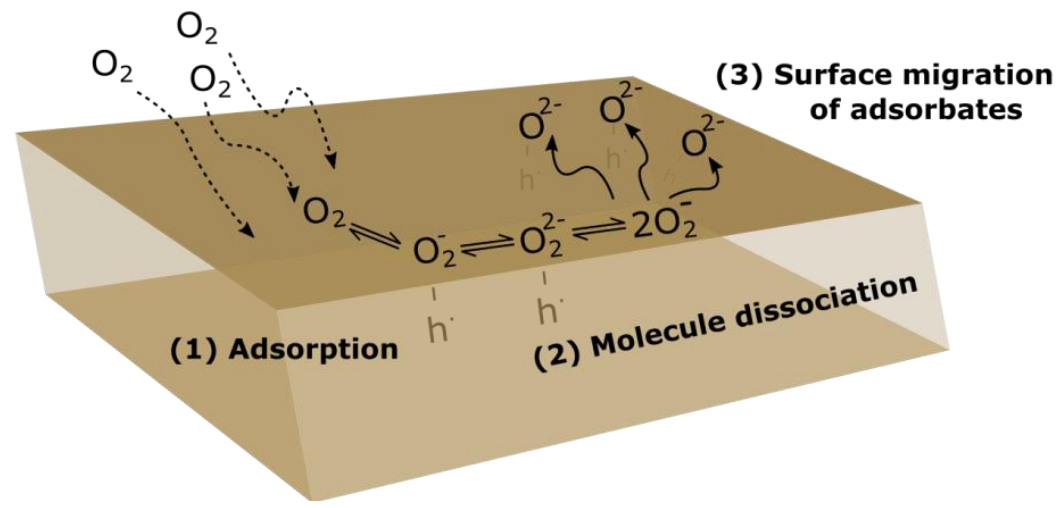

FIGURE 3.48: (a) Resistance ratio at room temperature as a function of the generated resistive state. (b) $\mathrm{T}_{\mathrm{MIT}}$ extracted from the $\mathrm{R}(\mathrm{T})$ curves for each resistive state.

These three steps draw a framework in which $\mathrm{O}^{-}$species are adsorbed at the surface with a certain coverage $\Theta$ which has a strong depedence on the $\mathrm{p}\left(\mathrm{O}_{2}\right)$.

Oxygen incorporation at the LRS $\rightarrow$ HRS transition: When a negative bias is applied, an electric field pointing downwards is generated between the tip and the film. Attracted by this intense electric field, $\mathrm{O}^{2-}$ species in the crystal accumulate at the interface. In addition, the electric field induces the following electrochemical reaction

$$
O_{x}^{O} \longrightarrow O_{\mathrm{ads}}^{2-}+V_{o}^{*}
$$

When the generated $\mathrm{O}_{\text {ads }}^{2-}$ (or $\mathrm{O}_{\text {ads }}^{-}$) is at the surface it needs to diffuse to meet another $\mathrm{O}_{\text {ads }}^{-}$to form an $\mathrm{O}_{2}$ molecule. As explained before, this is expected to be the RDS of the whole oxygen exchange reaction. When RS is performed under low $\mathrm{p}\left(\mathrm{O}_{2}\right)$, adsorption coverage is low and so the formation of $\mathrm{O}_{2}$ molecules will not be favoured because the surface diffusion length is much larger. As a consequence, at low pressures, the overall number of exchanged oxygen ions through the surface will be strongly reduced and therefore the $R_{\text {off }} / R_{\text {on }}$ ratio would heavily decrease, in agreement with what we observe in figure 3.46. Therefore, at low $\mathrm{p}\left(\mathrm{O}_{2}\right)$, the small resistance ratio obtained suggests that the MIT line is not crossed in this transition due to the small amount of ions exchange. When the oxygen ions are exchanged, oxygen vacancies are created into the system, which produces a concomitant decrease in the hole carrier density and a decrease of the oxidation state of in the Mn cation:

$$
L a_{1-x}^{3+} S r_{x}^{2+} M n_{1-x}^{3+} M n_{x}^{4+} O_{3}^{2-} y O_{a d s}^{2-}+\vec{E} \rightarrow L a_{1-x}^{3+} S r_{x}^{2+} M n_{1-x+2 y}^{3+} M n_{x-2 y}^{4+} O_{3-y}^{2-} V_{y}^{*}+2 y e^{-}
$$

consistent with a decreasing concentration of hole carriers, since LSMO compounds are hole controlled with a narrow band influenced by the strong correlation [73] (close to Mott insulators).

Oxygen excorporation at the HRS $\rightarrow$ LRS transition: At positive bias, the applied electric field points upwards. Therefore, the annihilation of the previously 
generated $\mathrm{V}_{o}^{*}$ in the system occurs through the inverse electrochemical reaction

$$
O_{\text {ads }}^{2-}+V_{o}^{*} \longrightarrow O_{x}^{O}
$$

Oxygen adsorbates at the surface are incorporated into the system via this electrochemical process and therefore, oxygen stoichiometry is restored. Note that this has an associated valence change in the Mn metal cations, which simultaneously increases the number of carriers leading the system to a more metallic conduction state.

The schematic view of both processes is illustrated in figure 3.49 .
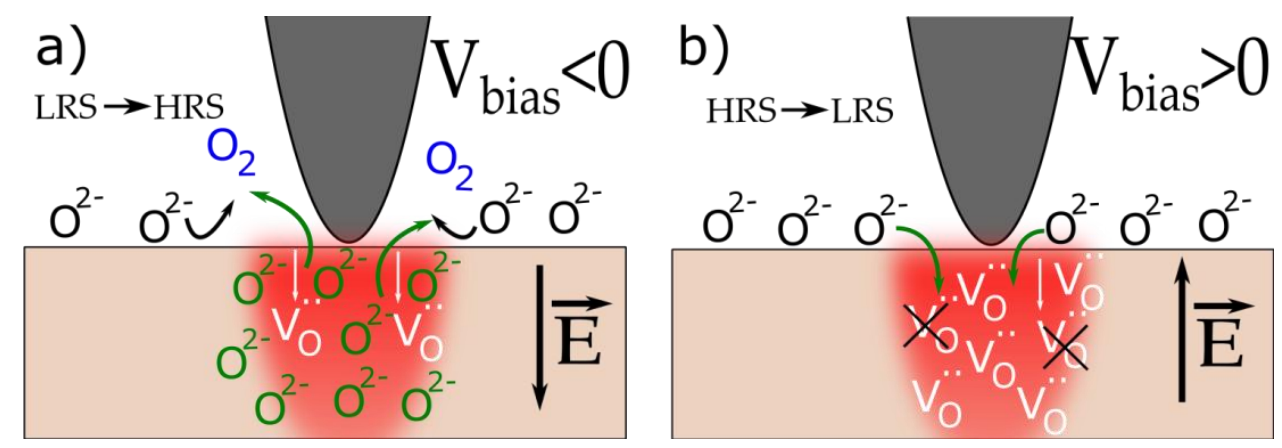

FIGURE 3.49: (a) Schema of the oxygen excorporation process. $\mathrm{O}_{2}$ from the crystal electrochemically react with an adsorbate to form an $\mathrm{O}_{2}$ molecule and creating an oxygen vacancy in the system. (b) Schema of the incorporation step. Oxygen adsorbates are driven into the crystal lattice annihilating an oxygen vacancy.

In summary, we have demonstrated the strong impact that external $p\left(O_{2}\right)$ has on the RS performance of LSMO films. The capabilities of the system to exchange $\mathrm{O}^{2-}$ with the ambient is heavily decreased when the external pressure is lowered. Introducing the experiments $p\left(\mathrm{O}_{2}\right)$ values in equation 3.4 leads to an $\mathrm{O}_{2}$ adsorbate surface coverage $\Theta$ decrease of a factor of $10^{2}-10^{3}$ at low pressure conditions $\left(P=10^{-6}\right.$ mbar $)$. This in good agreement with the decrease of the resistance ratio observed in that conditions. Therefore, the diffusion length that an adsorbate has to overcome to find another adsorbate and form an $\mathrm{O}_{2}$ molecule is larger, and therefore, the excorporation process is not favoured. This fact accounts for the smaller HRS found at low pressures. In the same way, we could have performed the initial LRS $\rightarrow$ HRS transition at high $\mathrm{p}\left(\mathrm{O}_{2}\right)$ to generate a large HRS and then, reducing the pressure. In such an experiment, we expect that the HRS would be now fix and the generation of subsequent LRS would depend strongly on the coverage, leading to pressure-dependent LRS. In other words, the oxygen adsorbate coverage strongly determines the capabilities of the system to exchange oxygen, either in the incorporation or the excorporation processes. 


\subsection{Proof of concept of 3-Terminal device}

In this section, a proof of concept of a 3-Terminal device based on the MIT-RS in LSMO thin films will be introduced. We will demonstrate the viability of these materials for novel devices beyond Flash technology. The designed 3-terminal configuration proof-of-principle show that performing RS under a well-controlled region (RS-gated) enables to tune the lateral conduction of the device, as demonstrated in section 3.3. This would enable the development of transistor like devices in which the Field Effect is substituted by RS. For the 3-T proof-of-principle configuration presented in this section, we will follow the same approach as for the atmosphere dependent measurements, i.e., one the macroscopic $\mathrm{W}$-Au tips will be placed in direct contact to the LSMO surface on a LSMO bridge to induce a HRS reversibly while its lateral conductivity will be measured.

\subsubsection{Device fabrication and operation}

Devices were fabricated in a $10 \mathrm{~nm} \mathrm{La}{ }_{0.8} \mathrm{Sr}_{0.2} \mathrm{MnO}_{3}$ grown on STO substrates. First an optical lithography process was performed to define the metal electrode $100 \times 100$ $\mu \mathrm{m}^{2}$ areas. Then, $60 \mathrm{~nm}$ of Au were sputtered onto the sample followed by a lift-off process in an ultrasonic bath of acetone for 15 seconds. A second lithography step is done to define the LSMO bridges. After that, the exposed LSMO film is etched by Reactive Ion Etching (RIE). An optical microscopy image of the device is shown in figure 3.50 (a). The dimensions of bridge are $100 \mu \mathrm{m}$ long by $25 \mu \mathrm{m}$ width. The bridge is connected to 3 electrodes, labelled as S (for Source), D (for Drain) and A (for Auxiliar). An schema with the two probe configurations involved in the measurement are depicted in the same figure.

a)

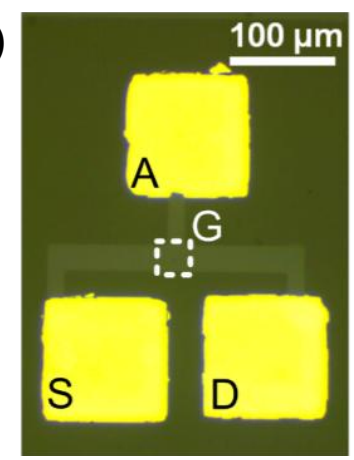

b)

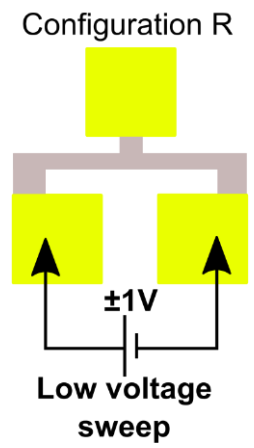

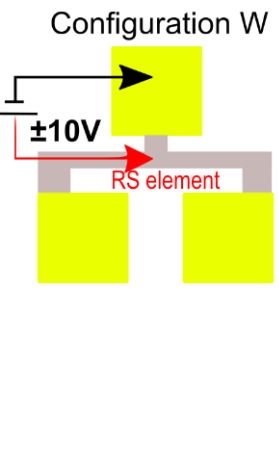

FIGURE 3.50: (a) Optical image of the 3T device. (b) Electrode configuration A. Low voltage sweep is applied between electrodes 1 and 2 to check the resistance state of the bridge (device). (c) Configuration B. RS is induced in the middle of the bridge by applying $\pm 10 \mathrm{~V}$ sweep. The active tip is in direct contact with the bridge and the second is placed in electrode A.

Measurements were performed in an Agilent 4156C Semiconductor Parameter Analyser using $4 \mathrm{~W}$-Au probes. Configuration R (R states for Reading) (electrodes $\mathrm{S}$, for source, and D, for drain) is used to measure laterally the resistance state of the bridge at low voltages $(\sim 0.1-1 \mathrm{~V})$. Low voltages are required in order not to disturb the resistance state in the bridge. Configuration $\mathrm{W}$ (W states for writing) is used to induce the RS effect in the middle of the bridge (G, for Gate). One of the tips is located in electrode A and the other is placed in direct contact with the 
bare LSMO surface in the bridge.This tip is not moved from its initial position during the whole experiment. For the device operation, the following measurement protocol is proposed:

Step 1: Configuration $\mathrm{R}$ - A sweep at low voltages is used to measured the pristine (LRS) state of the device.

Step 2: Configuration $\mathrm{W}$ - A sweep $0 \mathrm{~V} \rightarrow-10 \mathrm{~V} \rightarrow 0 \mathrm{~V}$ is applied to generate a HRS. Oxygen exchange with ambient induces the MIT below the tip and therefore the resistance of the bridge is increased.

Step 3: Configuration R - A sweep at low voltages is used to measured the resistance of the device in the HRS state generated in step 2.

Step 4: Configuration $\mathrm{W}$ - A sweep $0 \mathrm{~V} \rightarrow+10 \mathrm{~V} \rightarrow 0 \mathrm{~V}$ is applied to induce a LRS. Oxygen incorporation from ambient induces the reverse MIT and so, the resistance of the bridge is decreased.

Step 5: Configuration R - A Low voltage sweep is used to measured the LRS of the device.

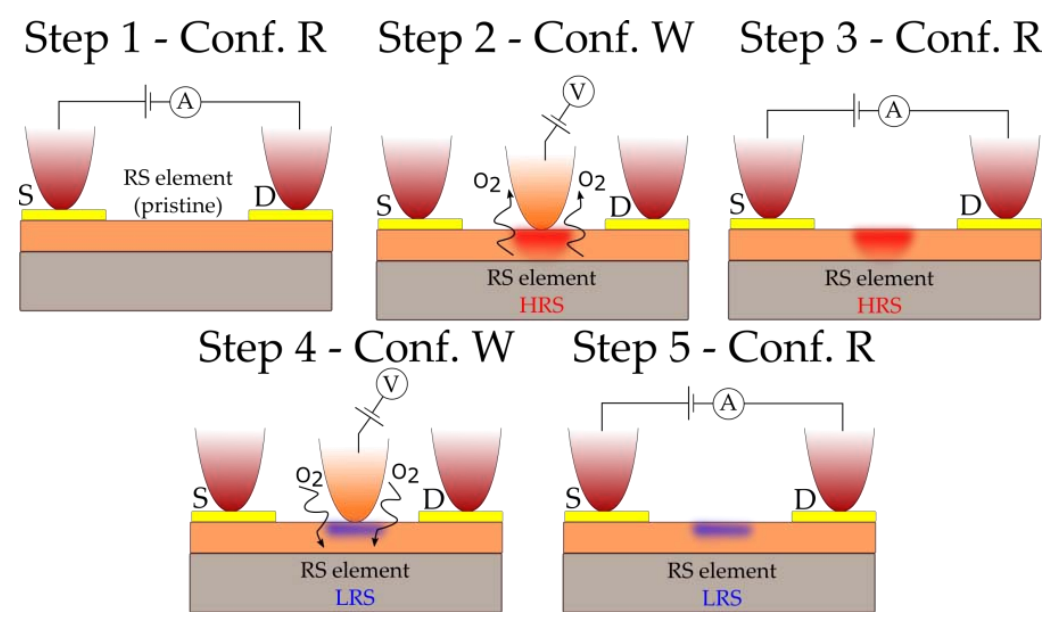

FIGURE 3.51: Schematic diagram of the 5 steps measurement protocol for the device operation. The lateral view of the device is depicted.

\subsubsection{Results and discussion}

The left image in figure 3.52 shows the resistance of the bridge measured at $\mathrm{V}_{\text {read }}=0.5 \mathrm{~V}$ in configuration $\mathrm{R}$ (steps 1,3 and 5). Right image shows the I-V sweeps performed with the tip in direct contact with the bridge (configuration $W$ ). Step $2(0 \mathrm{~V} \rightarrow-10 \mathrm{~V} \rightarrow 0 \mathrm{~V})$ is represented in black and step $4(0 \mathrm{~V} \rightarrow+10 \mathrm{~V} \rightarrow 0 \mathrm{~V})$ is represented in blue.

Configuration $\mathrm{W}$ is used to induce the RS effect in the middle of the bridge. The positive tip is located in electrode \#3 and the negative tip is placed in direct contact with the bare LSMO surface in the bridge. The initial resistance of the bridge is measured in configuration R with an initial resistance of $\sim 10^{6} \Omega$ (stated Step 1 in figure 3.52). After this, a first sweep (Step 2, black curve in figure 3.52) to induce a HRS in the bridge is performed. Then, a low voltage sweep is applied again in configuration $\mathrm{R}$. The result is plotted in 3.52 as step 3 . An increase of more than 2 
orders of magnitude is found $\left(\sim 10^{8} \Omega\right)$. To recover the LRS state, a positive sweep is applied in configuration W (step 4, blue curve in figure 3.52). The HRS $\rightarrow$ LRS is then induced in the bridge under the tip. Thus, a new low voltage sweep is measured in configuration $\mathrm{R}$ (step 5 in figure 3.52). A decrease of two orders of magnitude from the HRS is found and the initial value of the lateral resistance of the bridge is recovered.

a)

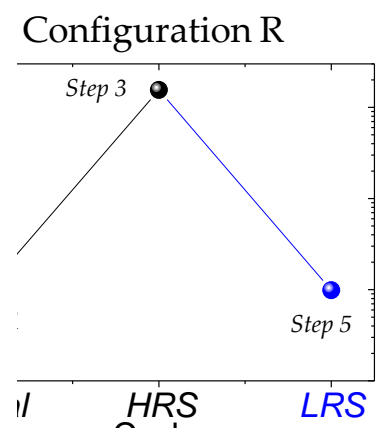

b) Configuration $\mathrm{W}$

FIGURE 3.52: (a) Measurements performed in configuration R. The resistance of the bridge is probed by applying a low voltage in the pristine (step1), HRS (step 3) and LRS (step 5). (b) Measurements performed in configuration $\mathrm{W}$. By the end of step $2(0 \mathrm{~V} \rightarrow-10 \mathrm{~V} \rightarrow$ $0 \mathrm{~V})$ the device is in its HRS. By the end of step $4(0 \mathrm{~V} \rightarrow+10 \mathrm{~V} \rightarrow 0 \mathrm{~V})$, the device switches back into the LRS.

In the following, we analyse the resistance values obtained from an electrical circuit point of view. The aim is to estimate the size of the volume that switches from LRS to HRS at the middle of the bridge induced by the tip of configuration W. Based on the exposed results, we assume that the full thickness of the film is transformed homogeneously below the $\mathrm{W}$-Au probe in this $10 \mathrm{~nm}$ film. This assumption is justified with our C-AFM experiments previously shown, where we demonstrated that by applying a $-10 \mathrm{~V}$ voltage a $10 \mathrm{~nm}$ film is fully transformed through thickness into a HRS. Therefore, we will estimate here the transformed area and check if it falls in the same order of magnitude as the expected tip size.

From a circuit point of view, the resistance along the bridge can be represented by three resistances in series (see figure 10). When the device is in the pristine or LRS state, the measured bridge resistance is $10^{6} \Omega$. The resistivity of the pristine LSMO can be deduced from the equation

$$
R_{L R S}=R_{1}+R_{2}+R_{3}=\rho^{L} \frac{L}{W \cdot t}
$$

Where $\rho^{L}, \mathrm{~L}, \mathrm{~W}$ and $\mathrm{t}$ are the resistivity, the length $(200 \mu \mathrm{m})$, the width $(20 \mu \mathrm{m})$ and the thickness $(10 \mathrm{~nm})$ of the bridge, respectively. In the formula, we have indicated three resistances: $R_{2}$ corresponds to the region of the bridge where the HRS will be later induced whereas $R_{1}$ and $R_{3}$ are the resistance of the lateral parts of the bridge. However, in the pristine state, we can introduce the values of the measured resistance, the width, the length and the thickness of the bridge to obtain its resistivity. A value of $\rho^{L}=10^{-3} \Omega \cdot m$ is obtained.

In the HRS, the region under the tip switches from a LRS to a HRS. Figure 3.53 shows schematically the lateral and top views of the device in the LRS and the HRS and their resistance model. For simplicity, let us assume that the tip has an 


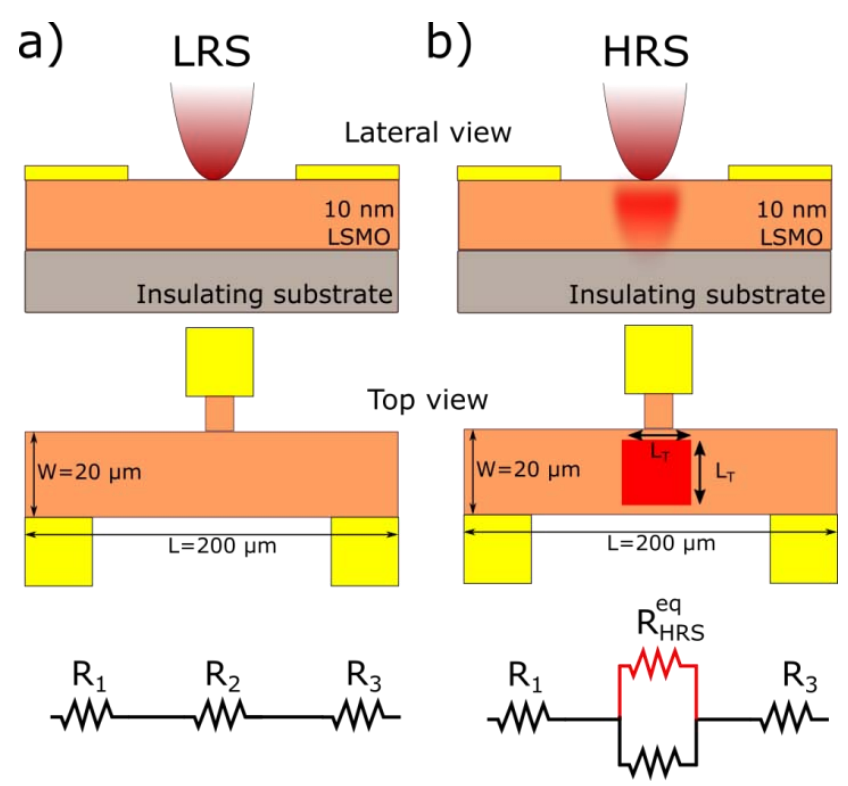

FIGURE 3.53: Lateral view, top view and equivalent resistance circuit of the bridge in: (a) Low Resistance State. (b) High resistance. In the HRS, the region below the tip has been transformed into a HRS with dimensions $\mathrm{L}_{T}$ by $\mathrm{L}_{T}$.

isotropic square shape of side $\mathrm{L}_{T}$ and when producing the switching, it induces a HRS in a square of dimensions $\mathrm{L}_{T}$ by $\mathrm{L}_{T}$ through the full thickness $\mathrm{t}$ of the film. In the following, we will estimate the area that has been transformed into the HRS, $\mathrm{L}_{T}$ and check whether it is a good approximation of the size of the tip. The resistance in the HRS can be expressed as

$$
R_{H R S}=R_{1}+R_{H R S}^{e q}+R_{3}=\frac{\rho^{L} \cdot\left(L-L_{T}\right)}{W \cdot t}+\frac{\rho^{L} \cdot \rho^{H} \cdot L_{T}}{t\left[\left(\rho^{L} \cdot L_{T}\right)+\left(\rho^{H \cdot} \cdot\left(W-L_{T}\right)\right)\right]}
$$

The first term of the equation corresponds to the sum of the serial resistance $R_{1}$ and $\mathrm{R}_{3}$. The second term expresses the equivalent resistance, $R_{H R S}^{e q}$. This equivalent resistance of the region under the tip is composed of two parallel resistances (a LRS part of dimensions $\left(\mathrm{L}_{-}-\mathrm{L}_{T}\right)$ by $\left(\mathrm{W}-\mathrm{L}_{T}\right)$ and a HRS part of dimensions $\mathrm{L}_{T}$ by $\left.\mathrm{L}_{T}\right)$. $\rho^{L}$ and $\rho^{H}$ are the resistivity of the bridge in the LRS and the HRS respectively. Equation ?? might be rewritten as

$$
R_{H R S}=\frac{\rho^{L} \cdot L}{W \cdot t}\left[1+\frac{L_{T}}{L} \cdot\left(\frac{1}{\frac{W_{T}}{W} \cdot\left(\frac{\rho^{H}}{\rho^{L}}+1\right)-1}-1\right)\right]
$$

From the measurement of the resistance in the HRS performed in configuration $\mathrm{R}$, we know that $R_{\mathrm{HRS}} \approx 10^{8} \Omega$. From the I-V sweeps performed in configuration $\mathrm{B}$, we can estimate the ratio between the resistivity of both states as $\rho^{L} / \rho^{H} \approx 10^{-3}$. For the sweep voltages used in the switching and the results obtained by C-AFM, we can assume that the full thickness of the film $(10 \mathrm{~nm})$ is transformed. Introducing all the numbers in the equation leads to a transformed length of

$$
L_{T} \approx 20 \mu m
$$

This value is consistent with the expected dimensions of the $\mathrm{W}$-Au probes used 
in the experiment plus it also shows that the full volume of the region $(20 \mu m \mathrm{x}$ $20 \mu m \times 10 \mathrm{~nm}$ ) under the tip was transformed into the HRS Therefore, the volume switching behaviour of these materials is also demonstrated by a 3-T configuration.

The aim of this experiment was to show a proof-of-principle device where the lateral conduction of the bridge in a 3-Terminal configuration has been modified by applying MIT-induced RS effect in the gate region. If a more realistic device is to be developed, oxygen exchange through atmosphere should be avoided. In that direction, during the development of this thesis we have demonstrated the benefit of using a bilayer system based on a ionic conductor like $\mathrm{CeO}_{2}$ which acts as an oxygen reservoir on top of the active LSMO layer.

A more realistic 3-T device was proposed by Dr. Rafael Ortega-Hernandez [248] based on the bilayer system $\mathrm{CeO}_{2} / \mathrm{LSMO}$. In the same way as proposed in this thesis, the lateral conduction of an $\mathrm{CeO}_{2} / \mathrm{LSMO}$ bridge was modified by inducing the RS in the gate element $\mathrm{Ag} / \mathrm{CeO}_{2} / \mathrm{LSMO}$, located at the middle of the bridge. By performing a similar circuit modelling of the different resistance contributions, a switching of the $40 \mathrm{~nm}$ active LSMO layer was demonstrated.

\subsection{Conclusions}

In this chapter,we have thoroughly investigated the resistive switching effect in $\mathrm{La}_{1-x} \mathrm{Sr}_{x} \mathrm{MnO}_{3}$ thin films. We have presented strong evidences supporting the link between the metal-insulator transition of $\mathrm{La}_{1-x} \mathrm{Sr}_{x} \mathrm{MnO}_{3}$ thin films and their RS behaviour.

We have demonstrated bipolar RS characteristics at the nanoscale by means of CAFM. Highly reproducible I-V sweeps show massive resistance ratios of $10^{3}-10^{5}$ between the pristine/LRS and the HRS with transition voltages in the range of $3-5 \mathrm{~V}$. Minor loops of the I-V hysteresis cycles are accessible by controlling the amplitude of the applied voltage in the pristine/LRS $\rightarrow$ HRS transition. Micrometric arrays of different HRS can be thus created by an area scan at different bias voltage. The LRS can be restored in these HRS micrometric regions by scanning at the opposite bias voltage. We have also shown that these multilevel resistive states are linked to volumetric transformation of the material in the region below the tip. This is demonstrated by performing a ring-shaped scan with the C-AFM tip at high bias, inducing a cylindrical shape HRS wall through thickness, thus isolating metallic pristine regions.

The composition of the material can be tuned to adjust the position of the MIT line, in such a way that it is closer to the operating temperature (room temperature). This strategy has lead us to to tune accordingly the RS parameters and to evaluate the electronic states of the HRS in different cases. The statistical study of local IV sweeps in $10 \mathrm{~nm} \mathrm{La}{ }_{0.8} \mathrm{Sr}_{0.2} \mathrm{MnO}_{3}$ and $\mathrm{La}_{0.7} \mathrm{Sr}_{0.3} \mathrm{MnO}_{3}$ thin films shows that the threshold voltages are smaller and with narrower distributions in the $\mathrm{x}=0.2$ case. This fact is linked to the closer proximity to the MIT at the operating temperature.

The same phenomenology observed at the nanoscale is reproduced when micrometric W-Au tips are placed in direct contact with the bare surface of the LSMO films. This strategy has been employed to perform I-V sweeps at different atmospheres to assess the influence of the ambient on the RS properties. We observed a huge decrease in the resistance ratio, from $10^{3}-10^{5}$ at ambient conditions to a 
factor 2 in high vacuum $\left(P=10^{-5}\right.$ mbar $)$. A general trend was observed, in which an increase of the oxygen partial pressure in the chamber produces an increase of the resistance ratio. The reduced ratio arises from of a lower HRS generated during the I-V sweep, while the LRS remains unaffected. This fact can be understood in terms of surface coverage modification of adsorbed oxygen species $\left(\mathrm{O}_{2}^{-}, \mathrm{O}^{-}\right)$upon changes in the $p\left(\mathrm{O}_{2}\right)$. The coverage value is decreased by several orders of magnitude when the total pressure is reduced from ambient to high vacuum conditions. The less adsorbates present at the surface, the less oxygen anions from the crystal structure can be exchanged through the atmosphere. This experiment support the MIT-induced RS scenario mediated by oxygen exchange with the ambient. Upon the application of the electric field, the simultaneous decrease in the number of holes (charge injection) and oxygen excorporation induce a valence change in the Mn metal cations, which drives the low resistance metallic system into a high resistance insulating state. The reverse process is induced when the electric field is reversed.

The generation of large HRS micrometric regions in the film has allowed us to perform ex-situ scanning tunnelling spectroscopy experiments in the same $10 \mathrm{~nm}$ $\mathrm{x}=0.2$ and $\mathrm{x}=.3$ samples. The HRS regions were generated at the different voltages $\left(V_{\text {bias }}=-5 \mathrm{~V}\right.$ and $\left.-7 \mathrm{~V}\right)$. By performing STM/S experiments in the modified and in the pristine regions, we have been able to estimate the change induced in the $\mathrm{Mn}$ valence. In both samples, a very similar change in the formal valence of the $\mathrm{Mn}$ ion is observed for a given HRS (generated at the same bias in both films). However, the relative change in the $\mathrm{Mn}$ formal valence leads to very different absolute values. The decrease in the number of available electronic states is larger in the film with $x=0.2$ that in that of $x=0.3$. The closer proximity of the former $(x=0.2)$ to the MIT line produces larger resistive changes than in the latter $(x=0.3)$.

The electrical response of HRS areas have been tested by 4-point configuration measurements as a function of temperature. We have shown that the sample modification to the HRS at high voltage, in a well-controlled bridge, leads to a full transformation of the volume of the bridge. After switching to the HRS, the resistivity at room temperature is increased by a factor of $10^{2}$ whereas the metal-insulator transition is shifted to lower temperatures. We have achieved changes in the transition temperature of $\Delta T_{\mathrm{MIT}}=117 \mathrm{~K}$. We have also demonstrated that the low resistance state might be recovered, showing a decrease of the bridge resistance and a shift of the $T_{\mathrm{MIT}}$ to higher temperatures, although full recovery was not achieved.

Finally, a proof-of-principle 3T configuration is proposed in which the lateral conduction of a LSMO bridge is modified by applying RS in a region located at the middle of the conduction bridge (gate). Reversible resistance changes of two orders of magnitude are found and a volume resistive switching is also demonstrated at the micrometric scale, thus paving the way to the development of transistor-like devices based on the RS approach. 



\section{Chapter 4}

\section{Resistive Swithching in other complex oxides}

In this chapter, our investigation on the RS properties of YBCO and RENO systems will be presented.

\subsection{RS behaviour in YBCO thin films}

\subsubsection{Introduction}

Although some works regarding the the bipolar resistive switching characteristics of YBCO can be found in the literature [284-291], there is still an open debate regarding its physical mechanism, due to the complexity of this compound. While Zhang et al. argues that the RS is produced due to charge trapping at oxygen vacancies [285], Acha et al. proposed oxygen migration as the main mechanism [286], localized at the metal/YBCO interface $[289,291]$. This latter argument is supported by Plecenik et al [284, 290].

In the following, we will present our studies of the RS properties of YBCO at the nanoscale studied by C-AFM. The origin of the RS will be explained in similar terms as in LSMO, i.e., in terms of Mott-like transition which can transform the bulk volume of the films. We will analyse the RS characteristics of bare YBCO and $\mathrm{CeO}_{2} / \mathrm{YBCO}$ surfaces. In addition, we will introduce a novel approach that might lead to reversible vortex pinning sites based on the RS effect.

\subsubsection{RS characterization in bare YBCO thin films}

As exposed in chapter 2, YBCO thin films of different thickness were grown onto STO insulating substrates by PLD. Our samples present smooth surfaces and excellent superconducting performance even for thickness down to 20-35 nm. In this section, C-AFM measurements regarding the RS behaviour of the films will be presented. A top-top configuration will be used with the tip grounded acting as a mobile electrode and the own film is used as the second electrode, with a silver wire firmly attached to the surface with silver paste. In addition, the influence of the resistive switching effect on the superconducting properties of the film will be evaluated by combining the C-AFM approach (to generate the resistive switching transition in the pristine YBCO matrix) and 4-point resistivity measurements. This approach might lead to novel fluxonic devices, in which vortex motion, and thus, 
the magnetic field dependence of the critical current of the superconductor can be modified.

\section{Electrical characterization by I-V sweeps}

The resistive switching properties of YBCO films were firstly investigated with I$\mathrm{V}$ sweeps performed by C-AFM. Figure 4.1 shows two I-V curves acquired on a $35 \mathrm{~nm}$ YBCO sample. The direction of the sweep is indicated by arrows. In the negative branch, the electric field points downwards whereas it points upwards under applied positive bias.

a)

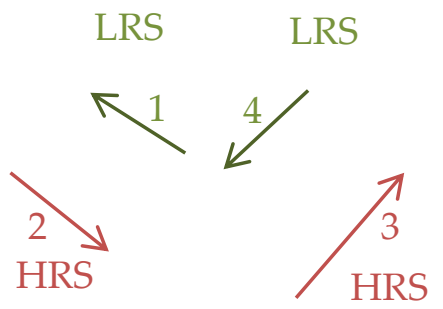

b)

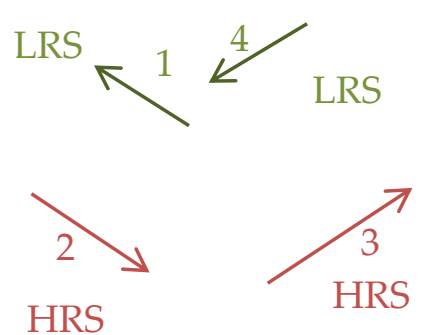

FIGURE 4.1: I-V curves in a $35 \mathrm{~nm}$ YBCO film. a) Lower bias I$\mathrm{V}$ sweep. b) Higher bias I-V sweep. The direction of the sweep is indicated by arrows.

The sweep is divided in 4 branches and starts towards negative bias (sweep 1, -3V (left) and $-4 \mathrm{~V}$ (right), respectively). The film starts a LRS and presents a smooth transition to a HRS. Then, in the sweep 2 the HRS is maintained in the excursion back to $0 \mathrm{~V}$. In the sweep 3 , towards positive bias ( $3 \mathrm{~V}$ and $4.5 \mathrm{~V}$ respectively), a sharp transition from the HRS to a LRS is observed. Then, the LRS is maintained in the excursion back to $0 \mathrm{~V}$ while sweep 4 . Even though the LRS $\rightarrow$ HRS transition is observed in the negative branch for the two sweeps. The initial LRS of the film in the loop shown in figure 4.1 (b) is more metallic than in (a). In both cases, the same HRS is generated in branch 2 . Therefore, in 4.1 (b) a higher resistance ratio $\left(10^{3}\right.$ instead of $10^{2}$ ) is induced.

Similarly to the model exposed in chapter 3 , at the LRS $\rightarrow$ HRS transition oxygen excorporates from the YBCO crystal structure upon the application of the electric field (negative bias). At the LRS $\rightarrow$ HRS transition (positive bias), oxygen excorporation occurs. A more detailed description of the process is provided later. In our view, the region below the tip in 4.1 (b) ("more metallic state") presents a larger oxygen content than in 4.1 (a) ("less metallic state"). These differences are ascribed to sample inhomogeneity. To support this point, more I-V curves are now presented in which the the initial state presents even an insulating state without showing RS behaviour.

Figure 4.2 (a) shows an I-V sweep acquired in the same conditions as described above. In the negative branch (steps 1 and 2) no hysteresis is observed. This means that the region below the tip is already in a HRS (oxygen-defficient region), and thus, by applying this sweep a higher HRS could not be achieved. In the positive branch (step 3) a transition from the initial HRS to a LRS is observed upon 
polarity inversion. This means that oxygen could be incorporated in the initial deoxygenated region inducing a HRS $\rightarrow$ LRS transition.
a)
b)
c)

FIGURE 4.2: Different scenarios for I-V sweeping.

a) Sweeping starts towards negative bias. b) Sweeping only towards positibe bias. c) Combination of both sweeping protocols to achieve reproducible RS in YBCO thin films.

The generated LRS has a non-volatile character. To prove so, unidirectional I-V sweeps were performed only towards positive bias sweeping $0 \mathrm{~V} \rightarrow 4 \mathrm{~V} \rightarrow 0 \mathrm{~V}$ several times at the same location (figure 4.2 (b)). The first sweep (in pink) starts in HRS and a sharp transition to a LRS is found at around 3V. This LRS is kept when decreasing the bias to $0 \mathrm{~V}$. By repeating this cycle the non-volatile character of the generated LRS can be tested. No hysteresis is observed in the subsequent cycles performed. To further prove the RS behaviour in the initial deoxygenated regime, the sweeping protocol was defined by combining first a positive sweep bias for the initial oxygenation of the region, and then the standard negative and positive sweeping bias exposed along this thesis. Figure 4.2 (c) shows the I-V sweep acquired with this protocol. The first sweep towards $+5 \mathrm{~V}$ (in pink) shows the initial HRS $\rightarrow$ LRS transition. The LRS is kept in the excursion back to 0V (step 2). Then, a negative bias to $-5 \mathrm{~V}$ is applied and a smooth transition from the LRS to the HRS is observed (step 3). The hysteresis is observed in the excursion back to 0V (step 4). Reversing the polarity to positive bias (step 5) induces again a HRS $\rightarrow$ LRS transition, which is kept in the excursion back to $0 \mathrm{~V}$ (step 6). With this measurement protocol, reproducibility in the I-V curves is highly increased.

In summary, to avoid effects due to oxygen inhomogeneities, first a positive bias sweep is recommended in order to incorporate oxygen in those oxygen-deficient homogeneous regions. In its pristine state, YBCO should present a metallic behaviour if properly oxygenated. An inhomogeneous oxygen content distribution would lead to local variations in several physical properties, such as electronic and ionic conductivities and superconducting critical temperature. In our case, the large variety of I-V curves found at the pristine state in the YBCO films is ascribed to an inhomogeneous oxygen content distribution close to the surface.

To address this issue, topography and resistance maps of a 35 and a $170 \mathrm{~nm}$ pristine YBCO thin films are shown in figure 4.3. The $35 \mathrm{~nm}$ sample (a) shows a flat surface with a RMS $=1.6 \mathrm{~nm}$. The $170 \mathrm{~nm}$ sample $(\mathrm{b})$ shows a strong presence of precipitates. Without taking them into account, the surface present a RMS of $2.3 \mathrm{~nm}$. Both resistances maps were acquired at $1.5 \mathrm{~V}$. The $\mathrm{R}$ scale of the maps has been set to its full scale. A large distribution of whiter regions is observed in both cases. However, no correlation between these white (insulating) regions and specific topographic features was found. This experiment confirms an inhomogeneous oxygen distribution at the surface of the film and some nm beneath. 
a)

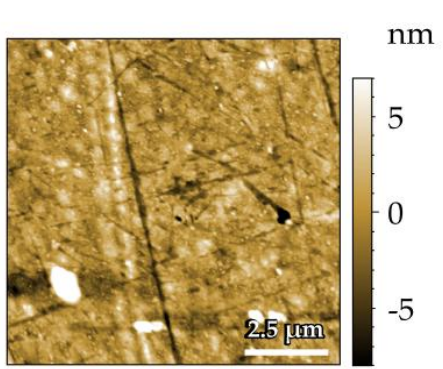

c)

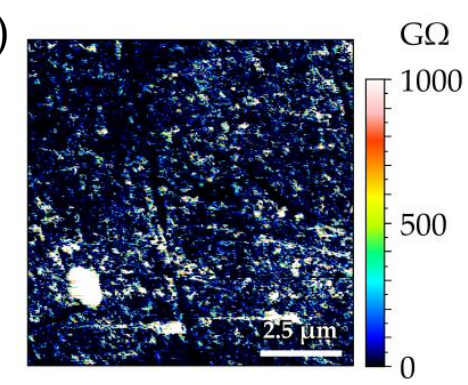

b)

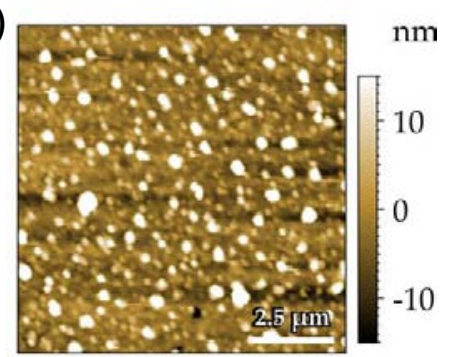

d)

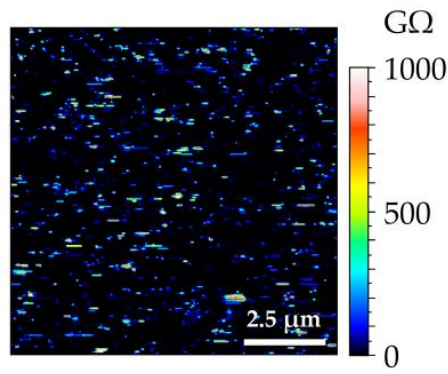

FIGURE 4.3: Topography and resistance map acquired at $1.5 \mathrm{~V}$ in (a and c) a $35 \mathrm{~nm}$ and (b and d) a $170 \mathrm{~nm}$ YBCO thin film in their asgrown state.

Several reports are found in the literature regarding the correlation of the microstructural defects in YBCO thin films and the higher critical currents achieved in these materials when compared to single crystals [292-294]. Generally speaking, YBCO thin films present a very complex microstructure with a rich defect landscape. Besides the aforementioned oxygen vacancies (probably close to the surface, figure 4.4), the presence of other intrinsic defects might have an impact in the RS behaviour of YBCO films. However, the role of microstructural defects (twin boundaries, stacking faults,...) on the RS phenomena is beyond the scope of this thesis, since a precise analysis of the different defects should be done.

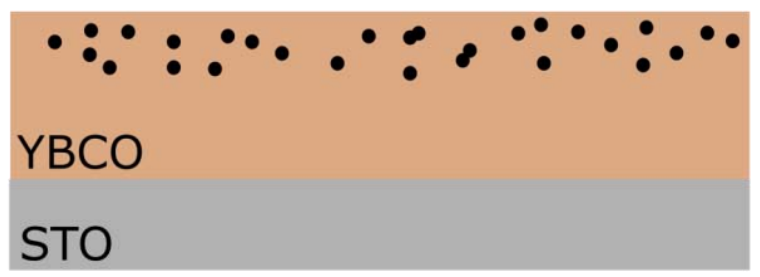

FIGURE 4.4: Schematic diagram of a YBCO pristine surface with higher density of oxygen vacancies close to it.

In conclusion, random variations in the local oxygen content close to the surface leads to different initial RS characteristics. This effect is minimized if first a positive bias, which induces oxygen incorporation into the initial oxygen-deficient region, is applied.

\section{Micrometic scale HRS areas induced by C-AFM}

A HRS state can be induced at the micrometer scale when the tip scans over a given area at high enough negative bias. To show the capabilities of YBCO systems to perform a controllable switch at the micrometric scale, a HRS state was induced 
by scanning a $2.5 x 2.5 \mu \mathrm{m}^{2}$ at $V_{\text {bias }}=-3.5 \mathrm{~V}$, and then the LRS was restored back at $V_{\text {bias }}=+3.5 \mathrm{~V}$. Figure 4.5 shows the full measurement. First (left), pristine topography and resistance maps of the area is acquired at $1.2 \mathrm{~V}$ (low voltage is used in order not to disturb the resistance state). As stated before, small HRS regions are already detected in the pristine state (white spots in the resistance map). Then, the voltage bias is set to $\mathrm{V}_{\text {bias }}=-3.5 \mathrm{~V}$. After this scab, the readout of the region is performed at $1.2 \mathrm{~V}$. Whiter region appear in the resistance map where the tip scanned at higher negative bias and therefore, inducing a HRS. No change is observed in the topography between pristine and the HRS.

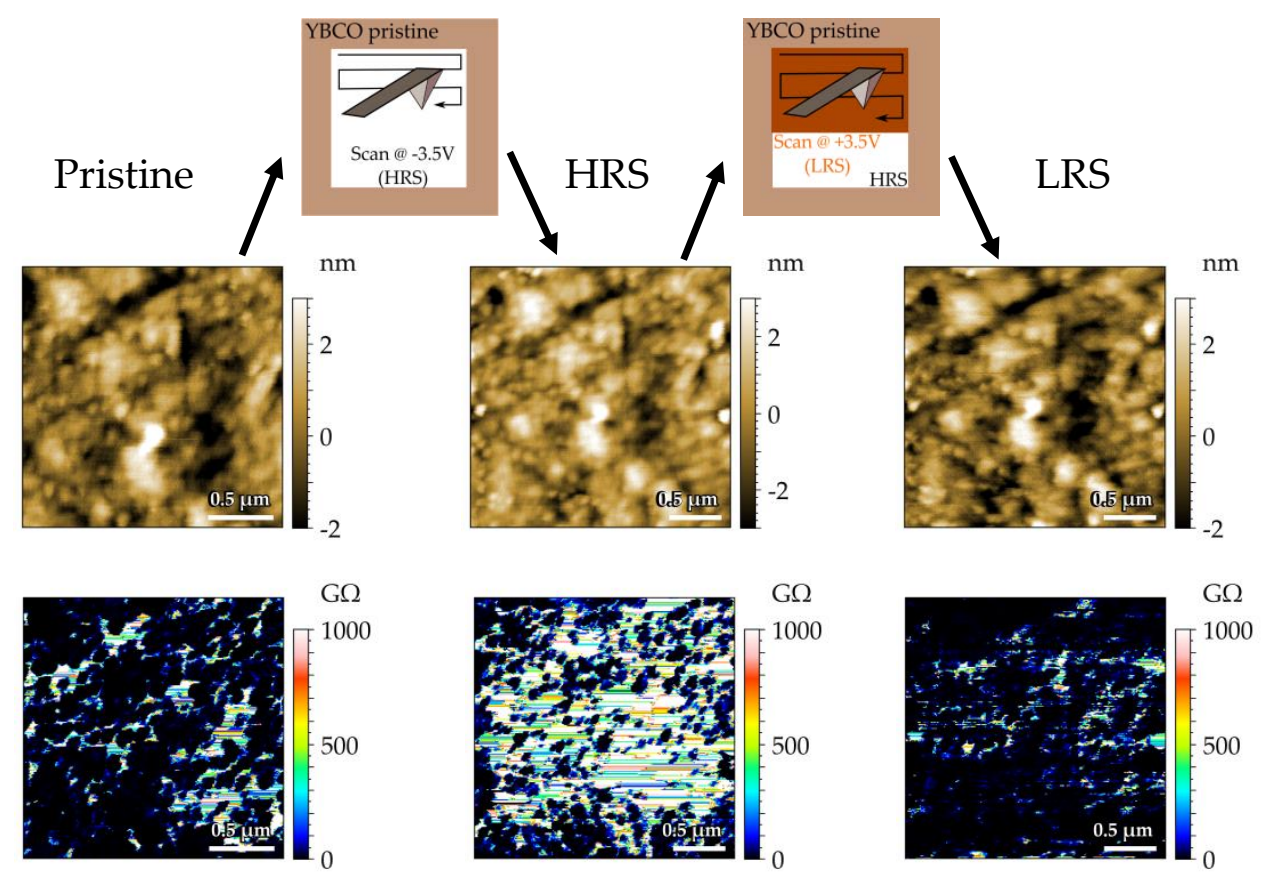

FIGURE 4.5: Sequential process to generate a complete RS cycle (pristine $\rightarrow$ HRS $\rightarrow$ LRS) at the micrometer scale.

To restore the LRS, a positive high bias should be used to scan the HRS region. In this case, a $V_{\mathrm{BIAS}}=+3.5 \mathrm{~V}$ is used to scan the region. After that, a low bias scan is again performed to read the generated LRS. As it is appreciated in the right image, the whole region appears in blue. Most of the HRS white regions observed in the previous map have changed their resistance state to a lower value (dark blue). In addition, the LRS presents lower presence of HRS regions than in the pristine state. This confirms that the initial oxygenation at the nanometric scale can be shifted to higher contents when a positive bias is applied. Topography in the LRS also remains unaffected. Reversibility is thus demonstrated at a micrometer scale.

For a better comparison, the resistance state of the three states has been extracted from the maps as follows. Each map has been transformed into a series of profiles $R_{x}(y)$. Then, whole set of profiles are averaged and thus an average profile of the resistance $\mathrm{R}_{\text {average }}(x)$ is obtained. The resulting profiles are plotted in figure 4.6.

The HRS presents 6-10 times higher average resistance value than the LRS and the pristine state. Although presenting similar values, the average resistance in the LRS is slightly lower than in the pristine. As said above, this fact supports the scenario of a YBCO surface with random variations in the local oxygen content 
FIGURE 4.6: Average resistance profiles obtained from the R maps in figure 4.5.

which leads to HRS nanometric regions inhomogeneously distributed. These areas can accommodate higher oxygen contents upon the application of positive bias.

One could wonder what is the minimum HRS feature size that could be generated with the C-AFM tip. This is of special interest for upcoming sections, where the influence of HRS regions on the superconducting properties of the films are investigated. HRS regions might have the ability to pin vortices, since different oxygent content generated in the LRS $\rightarrow$ HRS transition can generate superconductingdepressed regions which might act as a vortex pinning sites, which should be in the nm regime. To generate smaller HRS features, series of HRS dots were induced at $V_{\text {bias }}=-10 \mathrm{~V}$. The tip was holding its position for $100 \mathrm{~ms}$ at each dot in order to keep the same time scale as in the I-V sweeps. After that, a readout of the region was performed at $V_{\text {bias }}=1.2 \mathrm{~V}$, shown in figure 4.7. HRS dots of $\sim 200-250 \mathrm{~nm}$ are obtained. These features nicely correlate with the tip apex size, which has a radius of $\sim 100 \mathrm{~nm}$, and might induce larger features due to tip wear upon scans.

In conclusion, we have demonstrated the capabilities of this approach to generate micro- and nanometric HRS regions, where the superconducting properties might be modified and therefore could serve as a preferential vortex pinning site. This approach will be used in next sections to pattern superconducting YBCO bridges and to 'study their influence on the superconducting transition by means of $\rho(T)$ measurements.

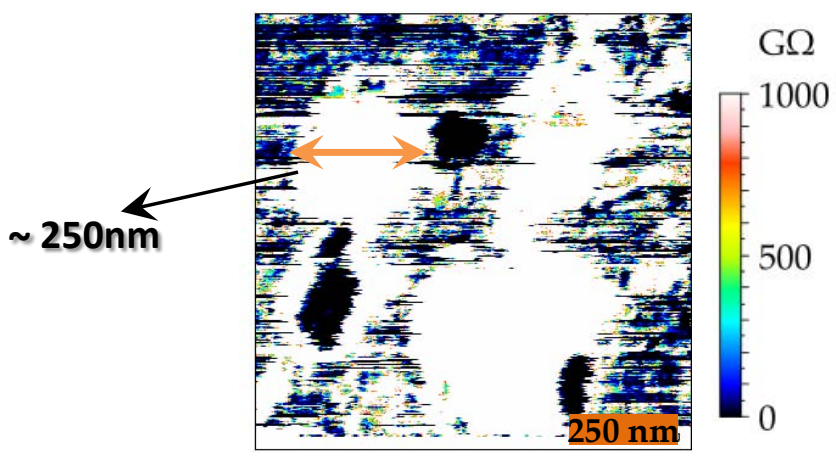

FIGURE 4.7: Array of HR dots generated at -10V. The tip was held at each position for $100 \mathrm{~ms}$. 


\section{Volume Resistive Switching in PLD YBCO thin films}

In the next experiment, rings at different negative bias were drawn with the AFM tip on the surface of a $10 \mathrm{~nm}$ YBCO sample. In particular,the bias used for drawing the rings were $-10 \mathrm{~V}$ and $-6 \mathrm{~V}$. As shown before, negative bias induce a HRS in the YBCO film. After the process, the region is scanned at $1.2 \mathrm{~V}$ to read the resistance modifications in the region. The results are shown in figure 4.8. The HRS ring generated at $-6 \mathrm{~V}(4.8(\mathrm{a}))$ shows an insulating state only where the tip has passed on, i.e., the contour of the ring. Inner and outter parts of the ring are electrically connected. However, the situation arising from the $-10 \mathrm{~V}$ case looks different. The inner part of the ring, which has not been modified, present an apparent insulating character. A bias of $-10 \mathrm{~V}$ is enough to transform the full thickness of the film $(\sim$ $10 \mathrm{~nm}$ ) into a HRS reaching the insulating substrate. In this way, the cylindrical inner part in the pristine state is topologically isolated from the YBCO metallic matrix by a thin HRS wall which blocks the current path.
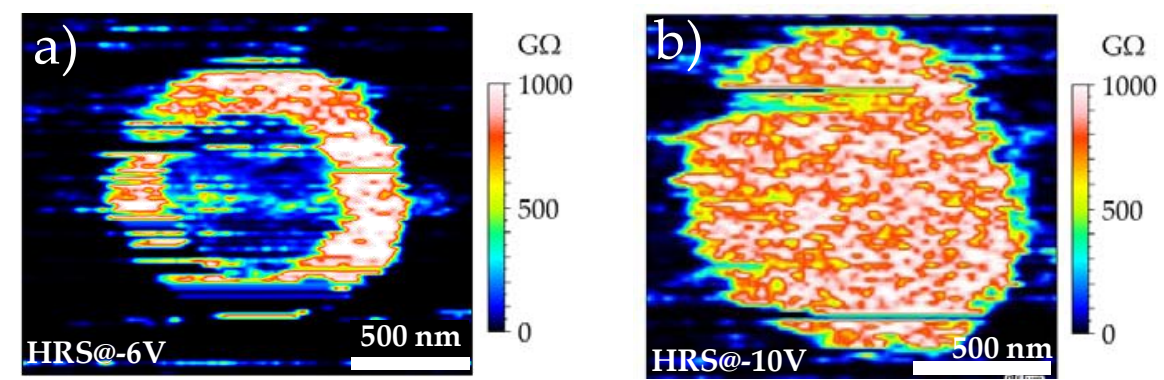

c)

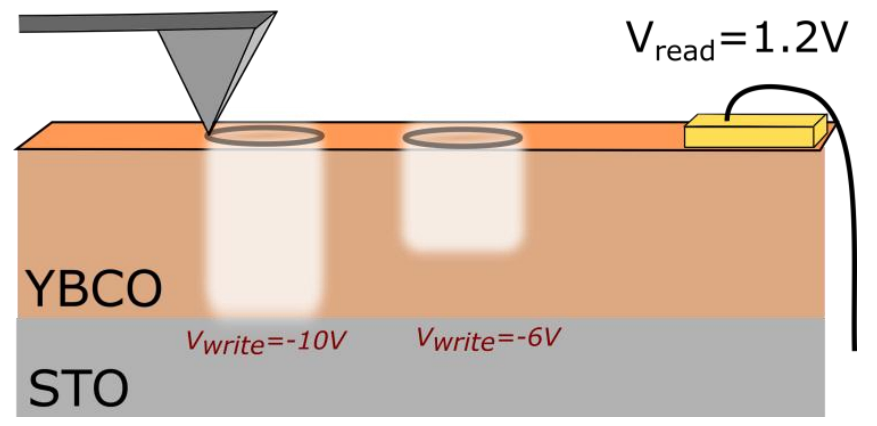

FIGURE 4.8: (a) Resistance map acquired at $1.2 \mathrm{~V}$ in regions where HRS rings were induced. (a) Ring performed at $-6 \mathrm{~V}$. (b) Ring performed at $-10 \mathrm{~V}$. (b) HRS rings where induced. (c) Schematic representation of both scenarios.

A schematic diagram of both scenarios is depicted in image c). It represents the acquisition of a low bias map to read out the resistance generated after drawing the rings. In the $-10 \mathrm{~V}$, where the full thickness of the film has turned into a HRS, the tip is not able to collect any current from the inner non-modified region because the HRS wall blocks it. However, when the tip scans on top of the $-6 \mathrm{~V}$ ring only the contour of the ring (HRS induced contour) shows an insulating character. Since the transformed thickness has not reach the insulating substrate, the current circulates between both electrodes through the bottom region of the film. Hence, we can confirm that the thickness of the generated HRS can be tuned with the amplitude of the negative bias. 
After all the experimental evidences shown up to now, the following mechanism responsible of the RS effect is proposed. Upon the application of a negative bias (electric field locally pointing downwards at the tip apex), $O_{\mathrm{YBCO}}^{2-}$ in the matrix are driven towards the interface, where eventually reacts with an adsorbate species $\mathrm{O}_{\text {Ads }}^{2-}$ to form an $\mathrm{O}_{2}$ molecule. Each oxygen exchanged from the YBCO structure to the ambient generates an oxygen vacancy in the system which leads to valence change in the $\mathrm{Cu}\left(\mathrm{Cu}^{3+} \rightarrow \mathrm{Cu}^{2+}\right)$ and a decrease in the number of hole carriers. In this way, a HRS is generated. To restore back the LRS state, a high electric field of the opposite polarity should be applied in order to induce the incorporation of $O_{\text {Ads }}^{2-}$ from the surface into the YBCO structure and the corresponding diffusion inside the YBCO matrix. This scenario is compatible with the observation of an inhomogeneous distribution of oxygen close to the surface in the pristine state, which leads to different conducting initial states and consequently, to a variety of I-V behaviours. However, this initial situation can be modified by applying a positive bias (electric field locally pointing upwards at the apex of the tip) field which should induce the incorporation of the $O_{\text {Ads }}^{2-}$ into the oxygen-deficient regions in the YBCO. We have confirmed that the thickness transformed into the HRS can be tuned with the amplitude of the bias voltage. Thus, a volume reversible switching effect is demonstrated in YBCO thin films.

\section{Transport studies of HRS arrays and its effect on the superconducting transition}

The superconducting properties of YBCO depends strongly on the oxygen content [295]. The superconducting state might be even suppressed below a certain doping. It has been also exposed that the capability of superconductors to transport electrical current without losses in the presence of magnetic fields rely on its ability to pin vortices (quantized flux). Vortices tend to be pinned in defects and nonsuperconducting regions. Hence, by combining the C-AFM approach to induce HRS at a local scale by modifying the oxygen content, the local superconducting properties of the film can be diminished and favour vortex pinning.

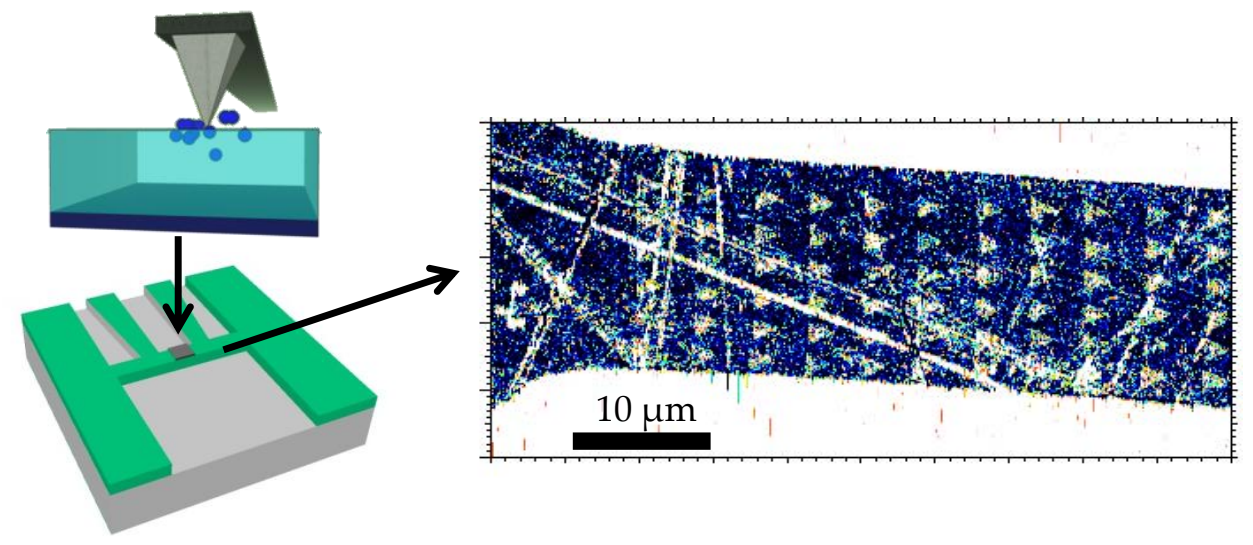

FIGURE 4.9: Diagram showing the HRS induction in a patterned YBCO sample. A resistance map with an array of HR regions is also shown.

These local modifications will be performed in a YBCO patterned bridge and 4point configuration measurements will be conducted as a function of temperature to study the superconducting transition. An schematic of the proposed process is 
shown in figure 4.9. In the patterned presented, we induced triangular (non- symmetric) insulating regions with interest in the study of vortex motion and ratchet effects [296].

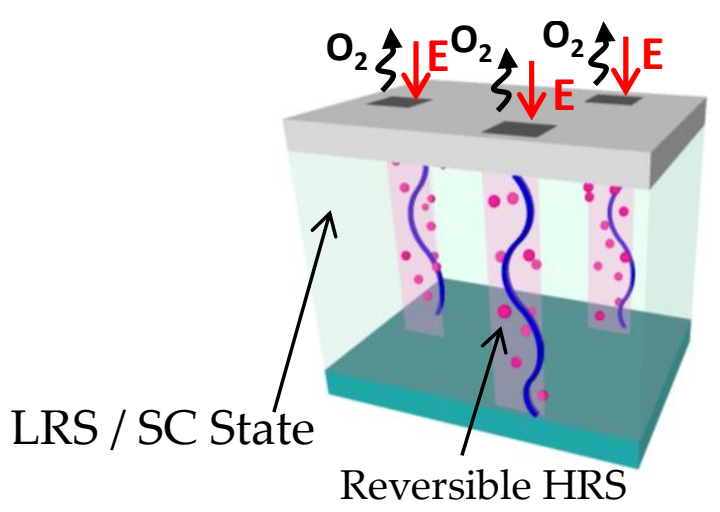

FIGURE 4.10: Schematic of an array of localized HRS nanometric regions where a vortices can be pinned.

Figure 4.10 shows an schematic picture of a situation in which locally HRS regions have been induced by C-AFM. If a magnetic field is applied to the modified sample, vortex can be accommodated at the HRS regions. The $J_{c}(H)$ dependence of the YBCO bridge can be reversible modified.

a)

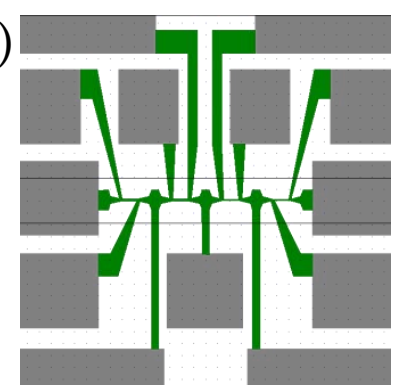

b)

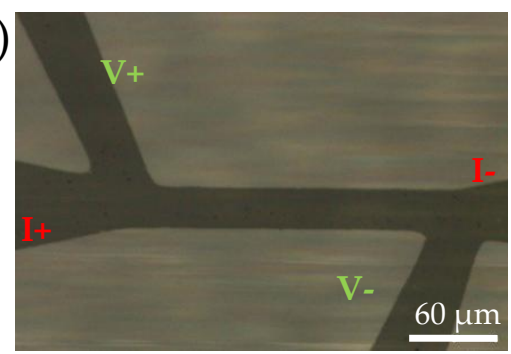

FIGURE 4.11: (a) Optical lithography mask used to pattern a pristine YBCO sample. (b) Zoom in one of the bridges obtained at the end of the process.

In the following, a description of sample preparation for 4-point measurements is performed. First, 300-500 nm of Ag metal electrodes are sputtered on the YBCO film by either shadow mask or optical lithography step followed by a lift-off process in acetone. Then, an annealing process at $450^{\circ}$ for 1 hour under $\mathrm{O}_{2}$ flux is performed. It serves to decrease the Ag contact resistance to allow measurements in the superconducting state, and to reoxygenate the sample to assure good superconducting properties. Then, the film is patterned by optical lithography to define the YBCO bridges (where RS effect will be induced). A wet-etching is performed to remove the exposed layer in $\mathrm{HNO}_{3}$ 1:100 for 5-20 seconds, depending on the thickness of the film. The used mask and one of the patterned bridges are shown in figure 4.11. The designed mask allows us to measure up to 4 different bridges in 4-point configuration. The size (length $\mathrm{x}$ width) of the bridge was varied from $100 \mu m \times 15 \mu m$ to $200 \mu m x 30 \mu m$.

The sequence of the experiment is as follows:

Step 1 - Resistivity measurement in the pristine state: The sample is mounted in the PPMS sample holder. Bridges were connected by Ag wire-bonding. The 
resistivity of the bridge in its pristine state is measured in the $300-50 \mathrm{~K}$ range to characterize the superconducting transition.

Step 2 - C-AFM nanostructuration: The patterned sample is mounted in the CAFM sample holder as described several times along this thesis. Then, the tip is placed in a bridge and a scan is performed. Then, the conditions of the HRS array are set (shape, distribution, voltage) and the nanostructuration is carried out. The readout of the nanostructured pattern is performed at $1.2 \mathrm{~V}$.

Step 3 - Resistivity measurement after nanostructuration: The nanostructurated YBCO bridge is mounted in the PPMS sample holder through Ag wire bonding. The resistivity is then measured in the range $300-50 \mathrm{~K}$ to study the transition between the normal and the superconducting state.

Step 4 - C-AFM safety check: After the measurement, the sample is taken back to the C-AFM to image again the bridge to check if any modifications could have taken place while measuring.

Two different arrays of HRS dots generated at $-10 \mathrm{~V}$ (as the ones shown in figure 4.7) were performed in two different bridges of a $100 \mathrm{~nm}$ YBCO sample. Namely, a higher density array and a lower density array. The same bias was applied in both cases, $-10 \mathrm{~V}$ for $100 \mathrm{~ms}$, while the geometry of the distribution was changed. This would lead to change in the transformed cross-section and therefore, the percolation paths of the superconducting current. The two arrays will be presented separately and a joint discussion will be shown afterwards.

\section{Higher density array}

In this first case, a square $4 \mu \mathrm{m}$ side array of HR dots was performed along the bridge of a $100 \mathrm{~nm}$ thick YBCO film. An scheme of the array, the topography map acquired after the nanostructuration and a profile extracted from the topography map are shown in figure 4.12.

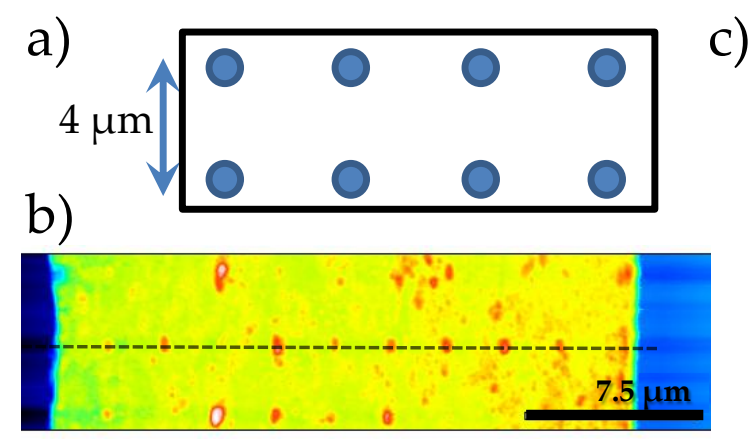

FIGURE 4.12: (a) Array of HRS dots generated at -10V. (b) Topography map after nanostructuration. (c) Profile extracted from the black dashed line in (b).

Small lifts in the topography due to the C-AFM nanostructuration was observed. Height lift of $\sim 30 \mathrm{~nm}$. The resistance map is not shown because it was not possible to distinguish the HRS dots from the intrinsic HRS small regions present in the pristine bridge. 
a)

b)

FIGURE 4.13: (a)Resistivity measurement of the nanostructurated $4 \mu m \times 4 \mu m$ array at $-10 \mathrm{~V}$. (b) Zoom of the measurement close to the SC transition.

After nanostructuration, the resistivity was measured again in the range $300-80 \mathrm{~K}$. The comparison of the bridge resistivity in the pristine state and after nanostructuration is shown in the figure $4.13 \mathrm{a}$. A dramatic increase in the resistance at room temperature (RT) is observed. A zoom of the superconducting transition close to $T_{c}$, is shown in figure $4.13 \mathrm{~b}$. While in the pristine state a sharp transition is found at around $\mathrm{T}_{c}=90 \mathrm{~K}$, in the nanostructurated case a shoulder-like shape is observed in the transition. A $3 \mathrm{~K}$ shift is found before reaching the noise level.

\section{Lower density array}

A second bridge of the same $100 \mathrm{~nm}$ sample was nanostructurated with a different array of dots. In this case, a rectangular array spaced $6 \mu \mathrm{m}$ was designed as indicated in figure 4.14. The same conditions were applied to generate the insulating dots, i.e., $-10 \mathrm{~V}$ during $100 \mathrm{~ms}$ at each dot. Smaller topography lift is also found in this case (10-15 nm, not shown), although the dots were not easily accessible due to the larger density of oxygen deficient regions in the pristine state of the bridge. Before and after nanostructuration, a resistivity measurement of the bridge varying temperature was performed. The plots are also shown in the same figure.

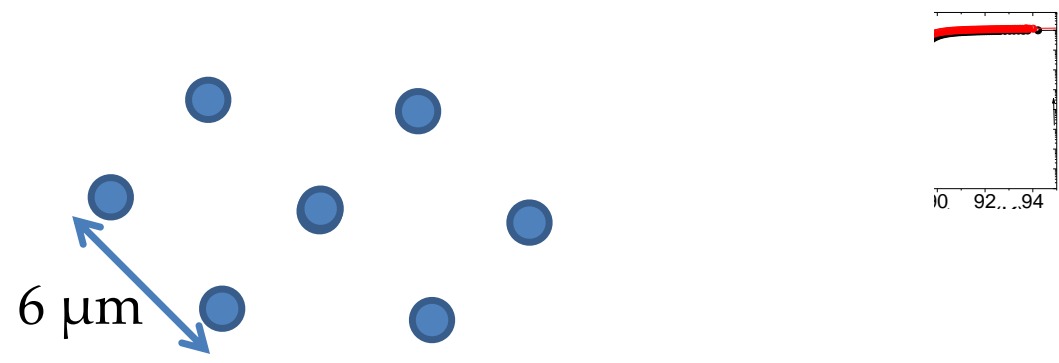

FIGURE 4.14: (a) Array of HRS dots generated at -10V. (b) Resistivity measurement of the nanostructurated $6 \mu \mathrm{m} \times 6 \mu \mathrm{m}$ array of $-10 \mathrm{~V}$. The inset shows a zoom of the measurement close to the SC transition

In this array, a smaller fraction of the cross section of the bridge is transformed into the HRS. Therefore, a smaller increase in the resistance and a less pronounced effect on the superconducting transition is expected. At room temperature, and increase of the resistance of $\sim 22 \%$ is found. In the inset, a zoom close to $\mathrm{T}_{c} \approx 90 \mathrm{~K}$ is 
presented. A slight decrease in the transition temperature of $\Delta T_{c} \approx 0.3 K$ is found between the initial and the HR states.

\section{Surface degradation}

After the resistivity measurements, the sample was left in air for some days. Eventually, surface degradation was observed in the YBCO bridges where the nanostructuration pattern were performed. Figure 4.15 shows an AFM topography map (a) (and a profile extracted from the map (b)) and an optical micrograph (c) of the higher density array bridge. Red regions have appeared on top of the bridge, as observed in the optical image. From the AFM measurements, we confirm that average height lift off $\sim 50-60 \mathrm{~nm}$ (note that the film thickness is $110 \mathrm{~nm}$ ).

a)

b)

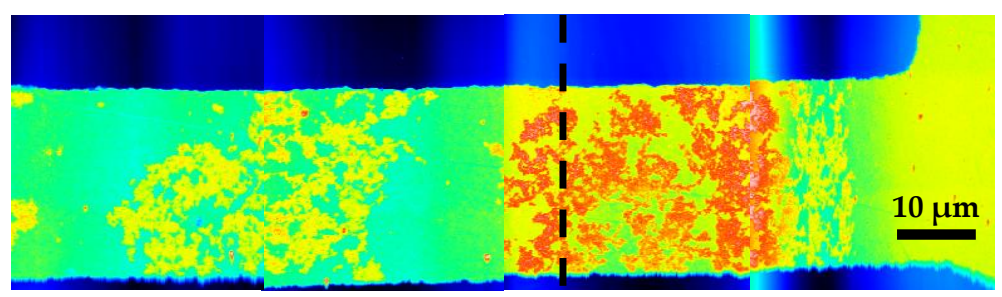

c)

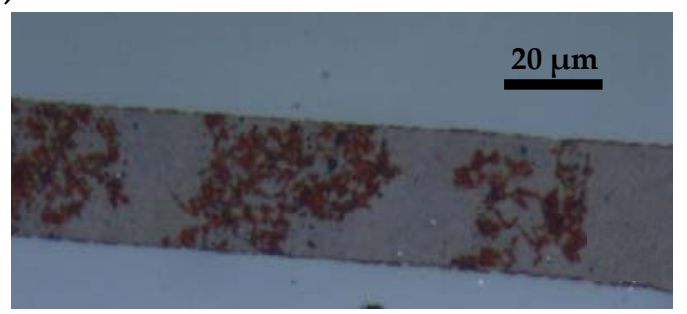

FIGURE 4.15: Evaluation of nanostructured HRS bridge after some days kept in air. (a) AFM topography map of the higher density array. (b) Profile corresponding to the dotted black line in (a). (c) Optical image of the same bridge.

Probably, the HRS regions are unstable under exposure to ambient conditions, and these HRS generated in the bridge were degrade by surface adsorbates, such as carbonate and hydroxyl groups in ambient conditions. This process can has a strong influence on the SC properties of the film and prevents further studies trying to recover the LRS. To avoid this situation, the use of a thin capping layer to protect YBCO film surface reveals crucial. With this pourpose, 3-5 $\mathrm{nm}$ of $\mathrm{CeO}_{2}$ have been ex-situ deposited by ALD on the YBCO films without affecting the superconducting properties of the film as shown chapter 2 ,

\subsubsection{RS characterization in $\mathrm{CeO}_{2} / \mathrm{YBCO}$ bilayer systems}

The use of a thin capping layer might avoid the degradation of YBCO patterned surfaces upon ageing. As shown in chapter 2, 3-5 $\mathrm{nm}$ of $\mathrm{CeO}_{2}$ was deposited by $\mathrm{ALD}$ at $275^{\circ} \mathrm{C}$ without any degradation of neither the surface nor the superconducting properties. To validate the RS properties of the $3 \mathrm{~nm} \mathrm{CeO}_{2} / 100 \mathrm{~nm} \mathrm{YBCO}$ bilayer systems, similar studies as the ones shown for YBCO are performed. In addition to the capping role that $\mathrm{CeO}_{2}$ might play, it is worth to remind its good 
ionic conduction and specially, the role that can play as oxygen reservoir, as demonstrated for $\mathrm{CeO}_{2} / \mathrm{LSMO}$ bilayers in this thesis.

\section{I-V curves and HRS maps induced by C-AFM}

First, the topography and its resistance map at $1.2 \mathrm{~V}$ of a pristine surface of the bilayer is shown in figure 4.16. Although similar HRS regions are observed as in the bare YBCO in the pristine state, their density is smaller and therefore, its initial resistance state seems slightly more homogeneous. This might be attributed to the capping effect of $\mathrm{CeO}_{2}$ layer, which prevents surface degradation and stabilizes the surface oxygen content. In addition, the presence of the $3 \mathrm{~nm}$ of ceria does not suppress the conductivity.

a)

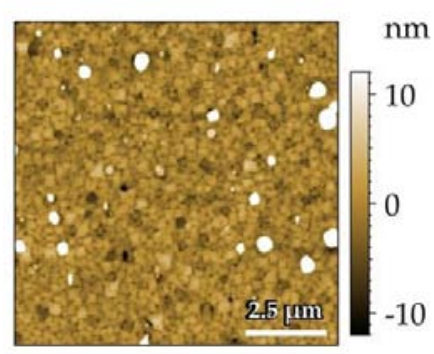

b)

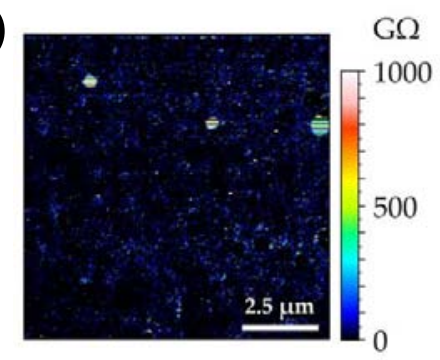

FIGURE 4.16: (a) Topography and (b) resistance map at $1.2 \mathrm{~V}$ of a 3 nm ALD-CeO $/ 100$ nm PLD-YBCO.

The same I-V behaviours as depicted for the bare $\mathrm{YBCO}$ case are still observed in the $\mathrm{CeO}_{2} / \mathrm{YBCO}$ bilayer. As an example, an I-V sweep towards negative bias with a LRS initial state is shown in figure 4.17 (a). The first sweep (branch 1) changes smoothly the LRS into a HRS state (-4.5V). The HRS is maintained during the voltage excursion back to $0 \mathrm{~V}$. Upon application of a positive bias, a transition from the HRS into a LRS is observed (branch 3), and this state is kept in the excursion back to $0 \mathrm{~V}$, completing the full RS cycle. Right image in the same figure shows the resistance ratios of the this I-V curve and the one obtained for the bare YBCO case (figure 4.1). Both are comparable since they were performed at intermediate voltages. The first remarkable evidence is that the $\mathrm{CeO}_{2}$ layer has an effect on the transition voltages. A higher bias is required in order to induce reversible switching. The presence of the thin dielectric layer, plus the high contact resistance between tip and $\mathrm{CeO}_{2}$ layer may be responsible of this effect. In addition, higher resistance ratios are obtained, since $\mathrm{CeO}_{2}$ capping layer might also boost the RS effect thanks to its good ionic conductivity. A comparison between the resistance ratio obtained in the bare YBCO and the bilayer case is shown in figure 4.17 (b). This fact will be further supported with more empirical evidences.

As previously demonstrated for the bare YBCO case, a full RS cycle was successfully induced at the micrometric scale by sequential scanning at adequate bias on a given region. The resistance maps (at $1.2 \mathrm{~V}$ ) of the full sequence are shown in figure 4.18. First, an image was acquired before any RS modification (left). Then, the scan size is set to $3 \mu \mathrm{m} \times 3 \mu \mathrm{m}$ and a $\mathrm{V}_{\text {Bias }}=-3.5 \mathrm{~V}$. A HRS state is induced in the region, as seen in the center image, acquired at the RS scan. The scan area is set to the HRS region, and a bias of $+3.5 \mathrm{~V}$ is applied. In this way, a LRS state is induced in the material. The resistance map after the process is also shown (right). The HRS is almost fully switched into the LRS. 
a)

b)

FIGURE 4.17: (a) I-V curve on a well oxygenated spot on the surface of the $\mathrm{CeO}_{2} / \mathrm{YBCO}$ bilayer system. (b) Resistance ratio for the I-V curve. For comparison, the resistance ratio for the I-V curve of bare YBCO is also shown.

The induced HRS presents a better homogeneity than the pure YBCO case (see figure 4.5) even though the presence of some non-switched grains. Both LRS and pristine images present very similar features. Furthermore, the resistance appears to have comparable magnitude in both the LRS and the pristine state (figure 4.18 (c)). Similarly to the analysis perform in the YBCO part, the maps where transformed into a series of profiles, which have been averaged in the horizontal direction. This was perform for both the topography (maps not shown because topography remains unchanged, as seen in the average plot in figure 4.18 (b)) and resistances (figure 4.18 (c)). In the latter plot, it can be seen that the HRS present higher average value than the pristine and LRS. In this case, LRS has a very similar profile to the pristine state. It is worth to remind than in the YBCO case, the LRS presents slightly smaller values than the pristine state. This further supports the idea of enhanced resistance ratios due to the good ionic conductivity of the $\mathrm{CeO}_{2}$ thin layer.

\section{Transport properties of HR arrays in bilayer systems.}

The study of transport properties of HR arrays in bare YBCO showed some issues regarding the surface degradation that takes place at some point during the whole process. In this section, similar studies as those presented for $\mathrm{YBCO}$ will be performed in a $3 \mathrm{~nm} \mathrm{CeO} / 100 \mathrm{~nm}$ YBCO patterned bilayer system. The double role that ceria plays (boosting RS effect and acting as a protecting layer) might avoid surface degradation and therefore, validate these kind of systems for further studies regarding the interplay between RS effect and superconductivity.

Samples were patterned as described in previous section. The same optical mask was used to define the measurement bridges. The same sequence as in the YBCO case was used as protocol measurement. Namely, a resistivity versus temperature curve, $\rho(T)$, of the bridge is firstly obtained in its pristine state. Then, the nanostructuration of the bridge is performed by C-AFM to induce a HRS. After that, a second $\rho(T)$ is carried out to analyse the effect of the induced HRS on the superconducting state of the film.

Two different arrays will be presented in this case. First, area scans at different bias will be induced in the bridges to analyse the effect on bias amplitude on the superconducting properties. The degradation of the surface will be also evaluated. 
a)

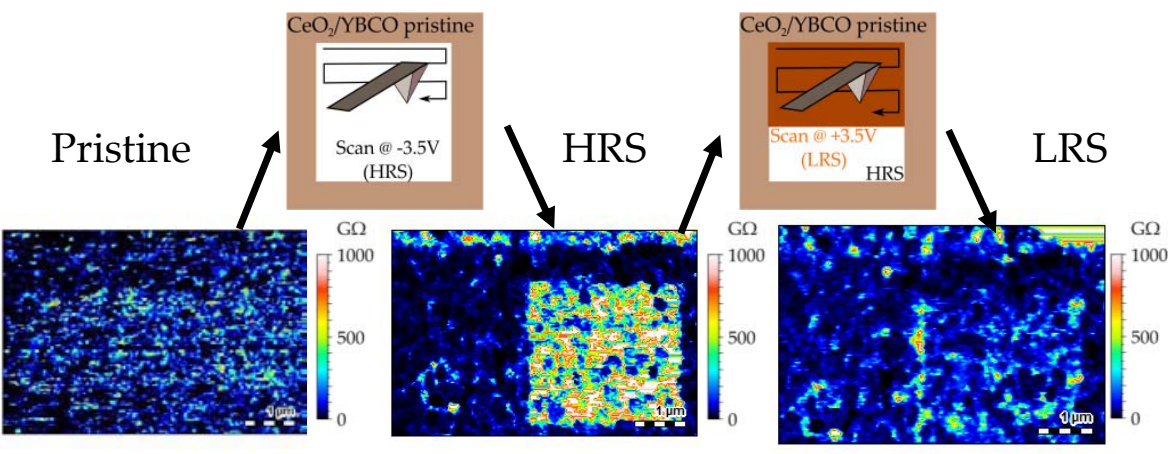

b)

c)

FIGURE 4.18: (a) Resistance maps of a sequential induction of a full RS cycle (pristine $\rightarrow$ HRS@-3.5V $\rightarrow$ LRS@+3.5V) in a $\mathrm{CeO}_{2} / \mathrm{YBCO}$ bilayer system. (b) Average profile of the topography for the 3 resistance states. (c) Average resistance profile for each state.

Second, a HRS array of dots at high bias will be used to analyse the pinning capabilities of this approach.

\section{Transport properties of HRS micrometric regions}

Once the $\rho(T)$ in the pristine state of the bridge was acquired, the nanostructuration by C-AFM was performed. By applying negative bias, a portion of the length and the full width of the bridges were transformed into the HRS. In particular, $\mathrm{V}_{\text {write }}=-4.5 \mathrm{~V}$ and $-10 \mathrm{~V}$ were used. Topography and resistance maps at $1.2 \mathrm{~V}$ were acquired after the nanostructuration and are shown in figure 4.19. The HRS regions are clearly seen for both the $-4.5 \mathrm{~V}$ (a) and $-10 \mathrm{~V}(\mathrm{~b})$. No damage in the topography is observed even for the $-10 \mathrm{~V}$ case.

a)

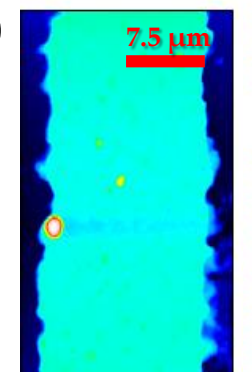

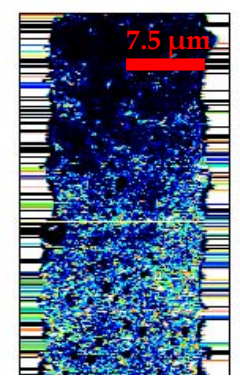

b)
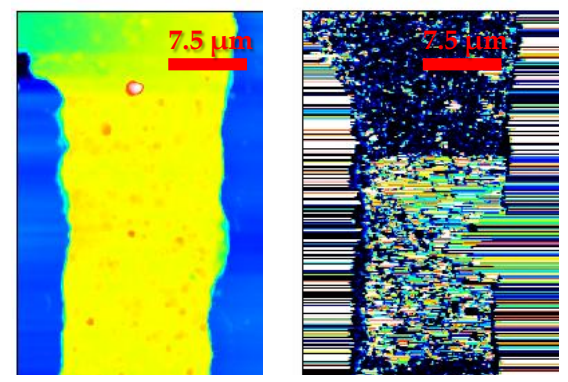

FIGURE 4.19: Topography and resistance maps at low bias of the bridges where HRS was induced in a piece of the bridge at (a) $-4.5 \mathrm{~V}$ and (b) $-10 \mathrm{~V}$. 
After the nanostructuration process, a second resistivity measurement of the bridges is performed. The pristine and the HRS $\rho(\mathrm{T})$ are shown in figure 4.20 for the the $-4.5 \mathrm{~V}$ scan (left) and right the $-10 \mathrm{~V}$ scan (right). In the former case, a slight increase of the resistance of $\sim 6 \%$ is found. In the latter, the increase of the $\rho$ at room temperature is larger and reaches $\sim 61 \%$. Insets in the figures show a zoom of the superconducting transition. Both bridges show $\mathrm{T}_{c} \approx 91 \mathrm{~K}$ in the pristine states. However, the HRS at $-4.5 \mathrm{~V}$ does not show any modification in $\mathrm{T}_{c}$. Contrary, in the $-10 \mathrm{~V}$ switched bridge some changes are observed. A shoulder-like broad transition is found, in which two different superconducting regions (with different $\mathrm{T}_{c}$ due to the different oxygenation induced by RS) are found.

a)

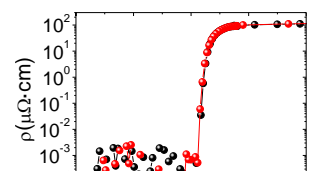

b)

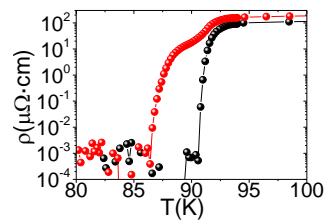

FIGURE 4.20: Resistivity plots of the pristine states and HRS at (a) $4.5 \mathrm{~V}$ and (b) $-10 \mathrm{~V}$ generated in different bridges of a $3 \mathrm{~nm} \mathrm{CeO}_{2} / 100$ $\mathrm{nm}$ YBCO film. Insets show a zoom close to the superconducting transition.

Compared to the pristine state, a shift of $3 \mathrm{~K}$ in the $\mathrm{T}_{c}$ is observed. After the measurement, an optical image was acquired to evaluate the degradation of the bridge (figure 4.21). No trace of degradation was found in any of the bridges.

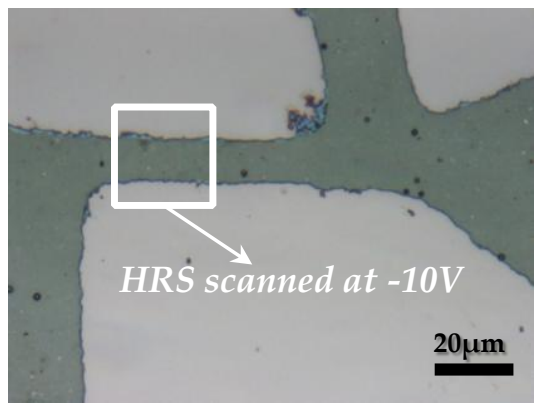

FIGURE 4.21: Optical image of the bridge with the HRS generated at $-10 \mathrm{~V}$ after the measurement process. No degradation is observed.

In this way, we fully validate the hypothesis that $\mathrm{CeO}_{2}$ acts as protecting layer against surface degradation in the HRS generated on top of the bilayer surface even for larger bias (-10V). Furthermore, we confirm the increase of the resistance if the bridge was tune with the amplitude of the applied voltage during the scan. To evaluate the pinning capabilities of this approach, HRS dots were generated in another YBCO bridge. 
Once surface degradation has been avoided, even at large and dense scans at $-10 \mathrm{~V}$, a more fine approach can be used to further investigate the potential of the RS approach to generate vortex pinning sites, that eventually would be reversible.

Similarly to the attempts performed in bare YBCO samples and shown in last section, an array of HRS dots will be performed along the bridge. After the pristine $\rho(T)$ characterization of the bridge, the HRS dots were induced in the same conditions as shown for the YBCO case, i.e., a rectangular array of $6 \mu \mathrm{m}$ side with a bias of $-10 \mathrm{~V}$ for $100 \mathrm{~ms}$ at each dot. The figure 4.22 shows an optical micrograph of the bridge (a) and the AFM topography map acquired before performing the nanostructuration (b). This time, no further maps were acquired after it to assure the minimum perturbation of the generated HRS.
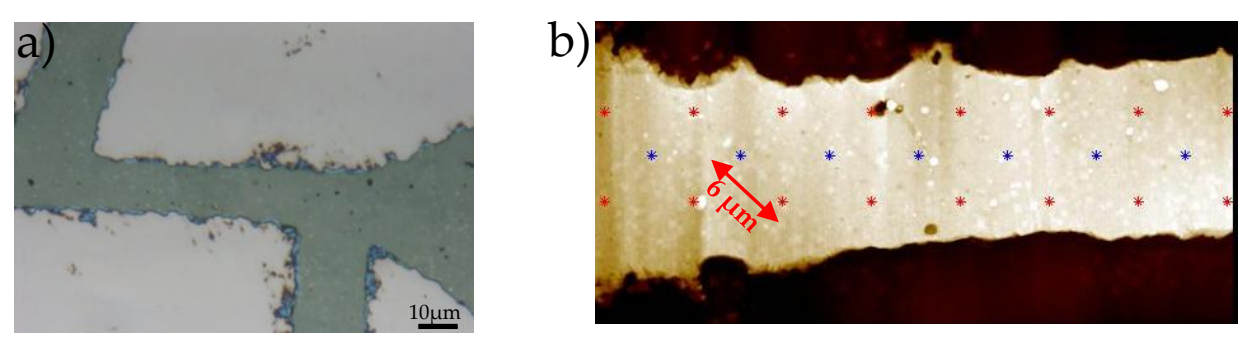

FIGURE 4.22: (a) Optical image of the bridge in its pristine state. (b) AFM topography map with the array of dots indicated in the figure.

After the nanostructuration process, a resistivity measurement is performed again and showed in figure 4.23 a. At room temperature, an increase of the resistivity of $\sim 10 \%$ is found. By zooming in the superconducting transition, a slight increase of $\mathrm{T}_{c}(\sim 0.3 K)$ is observed with no shoulder-like transition.

a)

b)

FIGURE 4.23: (a) Resistivity of the bridge for the pristine state and the generated HRS array of dots. (b) Zoom close to $\mathrm{T}_{c}$.

As a reminder, this same array was performed in the bare YBCO sample (figure 4.14). In that case, a larger increase in the resistance of the bridge was found for the bare YBCO bridge. The $\mathrm{CeO}_{2} / \mathrm{YBCO}$ bilayer system allows for a better control of the effect and sample degradation was avoided. To demonstrate so, an optical image of the picture days after this measurement is shown in figure 4.24.

After the full process, the surface topography does not have any trace of degradation. Therefore, this approach is validated for further assessment of the superconducting properties. In particular, the evaluation of the critical current density in the bridge as a function of magnetic field $\left(J_{c}(H)\right.$ was performed in the pristine and after the induction of the HRS dots array. $J_{c}(H)$ measurements were conducted at 


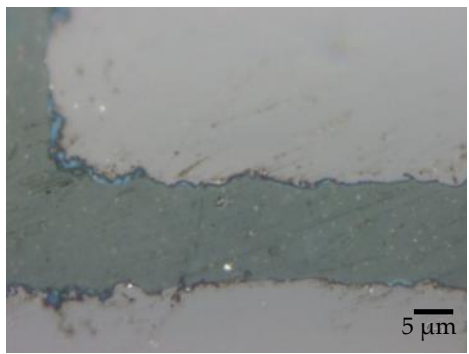

FIGURE 4.24: Optical image of the bridge where the HRS dots where induced taken after the measurement process.

different magnetic fields at $77 \mathrm{~K}$. I-V sweeps are performed to determine the critical current, $\mathrm{I}_{c}$, of the bridge (and hence, the critical current density, $\mathrm{J}_{c}$, since the geometry of the bridge is known) with the common criteria of $\mathrm{V}=1 \mu \mathrm{V}$. The obtained $\mathrm{J}_{c}(\mathrm{H})$ curves are shown in figure 4.25 .
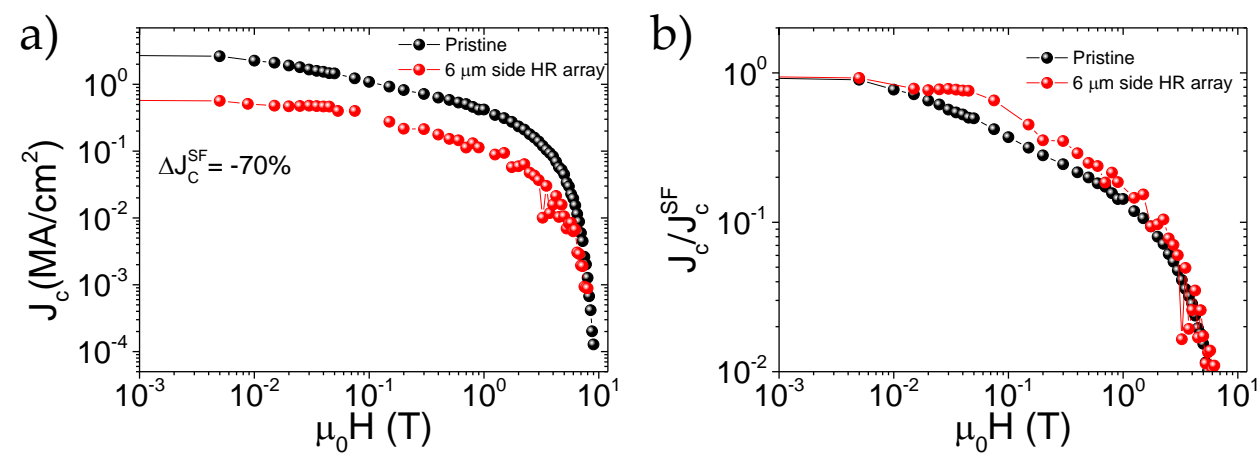

FIGURE 4.25: (a) $\mathrm{J}_{c}(\mathrm{H})$ dependence for the pristine and the HR dots array at $77 \mathrm{~K}$. (b) $\mathrm{J}_{c} / \mathrm{J}_{c}^{S F}$ vs magnetic field.

A larger and unexpected decrease of the critical current density is found for the HR dots array, close to the $70 \%$. It is important to remark that the same geometrical factors have been taken into account to derive the $\mathrm{J}_{c}$ in the pristine and in the HRS, although the cross section has been modified with the RS patterning. Due to the difficulties of knowing exactly which is the actual cross section, $\mathrm{J}_{c}$ curves were normalized to its $\mathrm{J}_{c}^{\mathrm{SF}}$ values to facilitate the comparison (b). The $J_{c}(H)$ curves show a similar round shape with an initial $\mathrm{J}_{c}(\mathrm{H})$ plateau with constant $\mathrm{J}_{c}$ value at low fields. This plateau (related with the number of the defects in the sample) is enlarged after the RS patterning. This means that the oxygen-deficient regions generated by the C-AFM approach might be suitable pinning sites and therefore, the dependence of the critical current under applied field might be tuned.

\subsubsection{Conclusions}

In this section, we have investigated the RS properties of bare $\mathrm{YBa}_{2} \mathrm{Cu}_{3} \mathrm{O}_{7-\delta}$ and $\mathrm{CeO}_{2} / \mathrm{YBa}_{2} \mathrm{Cu}_{3} \mathrm{O}_{7-\delta}$ thin films. In both cases, bipolar resistive switching characteristics are observed. An intense electric field generates a transition from the ON state to an OFF state by favouring oxygen exchange (excorporation from the YBCO matrix) with ambient, which generates oxygen vacancies in the system and inducing a valence change in the $\mathrm{Cu}$ cations. Conversely, the inversion of the electric field induces oxygen incorporation producing the opposite effects, thus inducing 
the $\mathrm{OFF} \rightarrow \mathrm{ON}$ transition and recover the initial LRS. Different I-V behaviours are found at different regions of the film, and each of them can be interpreted in terms of random variations of the oxygen content at the nanoscale. A positive sweep can be required to induce first oxygen incorporation into the initial partially (or totally) deoxygenated region showing a higher resistive state than the expected pristine LRS state. This ensures sample homogeneity in oxygen content and it increases reproducibility.

Micrometric arrays of HRS can be reversible induced even with no changes in the topography. Furthermore, controllable RS might occur (even through the full thickness of the film) upon variations on the bias amplitude. The same behaviours described for I-V curves are also observed at the micrometric scale. A negative scan induces the pristine LRS $\rightarrow$ HRS transition, and a positive bias scan at the same scanned region induces back the LRS. A positive bias scan onto a pristine region might also induce oxygen incorporation in small deoxygenated regions, thus modifying the initial higher resistive state to the expected LRS pristine state.

Arrays of HRS dots $(\sim 200 \mathrm{~nm})$ with different geometries have been induced in YBCO patterned bridges to analyse the influence of these HRS regions on the superconducting properties of the bridge. However, surface degradation have been observed in bare YBCO patterned bridges after nanostructuration, thus preventing a reliable analysis. This degradation has been avoided in the $\mathrm{CeO}_{2} / \mathrm{YBCO}$ bilayers with a capping of the 3-5 nm of ceria, which additionally enhances the RS performance of the films showing better reproducibility. In such a structure, we have assessed the influence of the micro- and nanometric HRS domains on the superconducting properties of the film. We have studied the influence of the bias amplitude on different non-superconducting regions transformed and also in patterned nanodots. Finally, we have demonstrated that our C-AFM approach shows very interesting capabilities towards the generation reconfigurable pinning sites to manipulate vortex at the nanoscale. Although further work should be done in this direction, the critical current density under applied magnetic field has been modified by this approach, confirming the pinning capabilities of HRS regions induced at the nanoscale. 


\subsection{Resistive Switching in $\mathrm{RENiO}_{3}$ systems}

\subsubsection{Introduction}

As exposed in chapter 2, we have focused our efforts on two systems, namely Chemical Solution deposited $\mathrm{RENiO}_{3}$ (RENO) $\left(\mathrm{LaNiO}_{3}\right.$ (LNO) and $\mathrm{NdNiO}_{3}$ (NNO)) grown on LAO substrates. We showed in chapter 2 that CSD LNO films does not present any temperature induced MIT and stays metallic in the full T range for the different thickness studied in this thesis. Temperature induced MIT has been reported in sputtered ultrathin films [100]. However, a MIT can be induced in this system upon stoichiometry changes [160] and by electric field-effect [297]. Contrary, NNO film present a MIT already in the bulk form, which is an indication of the more complex correlated electronic structure than LNO. In this chapter, the study of the resistive switching properties in RENO thin films will be presented. The growth process and the structural characterization of the films has been exposed in Chapter 2. A strong influence of the defects on the RS properties will be demonstrated. For comparison, a collaboration was started by the end of this Thesis with Mrs. Sara Catalano, Dr. Marta Gibert and Prof. J.M. Triscone from the University of Geneva. They provided us with RENO thin films grown by RF-sputtering with less density of defects than our CSD films. RS results on these samples will also be shown in this section.

The RS capabilities of RENO systems remains barely explored. However, some recent works from Ramanathan et al. have shown the promising potential of these systems due to its intricate electronic structure [298, 299]. A colossal resistance change was reported in $\mathrm{SmNiO}_{3}$ by electron doping in a reversible way. Furthermore, synaptic transistors were also fabricated with $\mathrm{SmNiO}_{3}$ films [298].

\subsubsection{RS characterization in $\mathrm{RENiO}_{3}$ grown by CSD}

\section{Electrical characterization by I-V sweeps}

To test the electrical characteristics in LNO and NNO thin films grown on LAO substrates, the same C-AFM configuration exposed along this thesis was employed, i.e., a top-top configuration in a low humidity atmosphere $(\mathrm{RH}<6 \%)$ with the the conducting tip acting as the active electrode and the own metallic RENO as counterelectrode. The switching hence occurs in the region below the tip.

Different behaviours in the I-V curves obtained by C-AFM are observed for CSD RENO systems. As explained in the YBCO case, a positive bias is usually required (but not always) to initialize the RS properties of the film. The following experiments were realized in $25 \mathrm{~nm}$ NNO thin films grown by CSD. Same features are observed in LNO thin films, as it will be shown in an example illustrating the RS behaviour in LNO, presented at the end of this section.

The first I-V sweep presented in figure 4.26 (a) corresponds to an unidirectional positive bias protocol $(0 \mathrm{~V} \rightarrow+10 \mathrm{~V} \rightarrow 0 \mathrm{~V})$ with several cycles. The first branch of the sweep is indicated in red. A transition from a more resistive state to a low resistance state is found. Once this first step occurs, the resulting LRS is maintained in the subsequent cycles. In (c), the resistance ratio derived from the I-V cycles in (a) is plotted. The ratio has been calculated by dividing the measured I values at every 
voltage step in the two branches of the cycle, i.e., Ratio $(V)=\frac{I(V)_{0 V \rightarrow+10 V}}{I(V)_{+10 V \rightarrow+0 V}}$. The ratio belonging to the first cycle is also shown in red. A first change of 3-4 orders of magnitude is obtained followed by a steady LRS unaltered by further positive bias sweeping.

a)

b)

c)

d)

FIGURE 4.26: (a) I-V sweeps where only positive bias was applied. An initialization step is required to set the region in its LRS. Subsequent cycles does not alter the LRS state. (b) I-V sweep of a full RS cycle. The initial state is marked in red. A full BRS cycle is observed with a ratio 5 orders of magnitude. (c) and (d) shows the resistance ratio derived from the I-V sweeps in (a) and (b) respectively.

A full RS cycling is shown in figure $4.26 \mathrm{~b}$. The applied protocol was as follows: $0 \mathrm{~V} \rightarrow+6 \mathrm{~V} \rightarrow 0 \mathrm{~V} \rightarrow-6 \mathrm{~V} \rightarrow 0 \mathrm{~V} \rightarrow+6 \mathrm{~V} \rightarrow 0 \mathrm{~V}$ for several times. The first positive sweep is indicated in red. As in image (a), the first sweep switches the system from a more resistive initial state to a fully LRS. This state is maintained in the excursion back to OV (step 2). Then, a negative bias is applied (step 3). In this branch, a LRS $\rightarrow$ HRS transition is found. The induced HRS is kept in the excursion back to 0V. Finally, a positive bias is again applied (step 5). The reverse transition, HRS $\rightarrow$ LRS occurs. The LRS is then fully recovered and kept in the excursion back to $0 \mathrm{~V}$. The sweep is repeated several times to check repeatability. The initial state (in red) is more resistive than the LRS but less than the induced HRS. The resistance ratio of this cycling is shown in the bottom part of the figure. After the initialization process (in red), a reproducible ratio $\sim 10^{4}-10^{6}$ between the HRS and the LRS is found, showing the great potential of these materials as memristor elements.

It is worth to remind that the RENO systems are metallic at room temperature. Therefore, an initial metallic LRS is expected and thus the first sweep towards positive bias to set the active region below the tip into a LRS would not be necessary in an homogeneous metallic film. In fact, points in where I-V sweeps show a fully metallic LRS initial state can also be found. Figure 4.27 (a) shows an I-V sweep acquired in a metallic point with the same protocol as the previous case, i.e., $0 \mathrm{~V} \rightarrow+10 \mathrm{~V} \rightarrow-10 \mathrm{~V} \rightarrow+10 \mathrm{~V} \rightarrow 0 \mathrm{~V}$ repeated several times. The resistance ratio obtained from the I-V sweeps is also shown in the figure 4.27 (b). The first sweep 
and the subsequently generated LRS states are very similar. In the resistance ratio, the first sweep (back and forth) indicated in red presents a ratio close to 1, which means that no initialization was needed since the initial state was already fully metallic. Then, in the negative bias, the $\mathrm{ON} \rightarrow \mathrm{OFF}$ transition is achieved. The HRS state is kept until the polarity is reversed and a sharp OFF $\rightarrow \mathrm{ON}$ transition occurs, which brings the region back into the LRS state. As observed in the resistance ratio, in this case two orders of magnitude between states is achieved. The diversity observed on the resistance ratios later, but we anticipate that these films posses an important density of structural defects that may directly contribute to the electrical conductivity.

a)

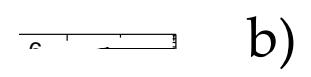

FIGURE 4.27: (a) I-V sweep starting towards positive bias (in red). Then a full $\pm 10 \mathrm{~V}$ sweep is performed. (b) Resistance ratio calculated from (a). The first sweep (in red) shows that the initial state is fully metallic and no initialization is required.

When the tip is placed in regions with a fully metallic state, no initialization is required, and therefore, the initial cycles could be started directly towards negative bias, where the LRS $\rightarrow$ HRS transition occurs. This would be the ideal case and large hysteresis can also be induced in the I-V curves. An example of such a loop is shown in figure 4.28 (a). The sweep starts towards negative bias (indicated in red) and the $\mathrm{ON} \rightarrow \mathrm{OFF}$ transition starts at $V \sim-2.5 \mathrm{~V}$. The HRS is maintained until the polarity is reversed. At positive bias, the $\mathrm{OFF} \rightarrow \mathrm{ON}$ transition takes place and the LRS is achieved. The cycle is then repeated several times. The resistance ratio (figure 4.28(b)) shows a colossal change of $10^{5}$ between the two resistive states, as also observed in figure 4.26.

a)

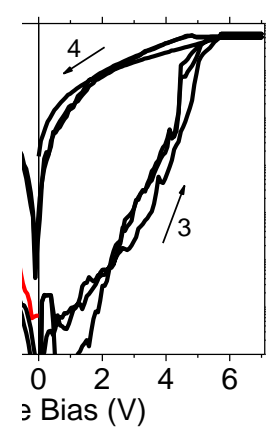

b)

FIGURE 4.28: (a)I-V sweep starting towards negative bias in a $25 \mathrm{~nm}$ NNO thin film and its resistance ratio (b).

This example reflects the ideal situation of a pristine region turned into a HRS by RS with large resistance ratio in RENO systems. However, as shown above and will be discussed later, the structural defects present in the film might strongly alter its 
RS capabilities, which may lead to the different I-V behaviours and disparity in the resistance ratio and transition voltages, as observed experimentally in figures 4.27 (a) and 4.28 (a).

As mentioned before, the same features in the I-V curves obtained for the NNO are observed in LNO thin films. As an example, a full RS cycle starting towards positive bias performed on a $25 \mathrm{~nm}$ LNO thin film is shown in figure 4.29. The same initialization process is required to enable the RS cycling (in red). Then, negative bias induced a transition from the LRS to the HRS. The reverse polarity produces the opposite effect, inducing the HRS $\rightarrow$ LRS transition. Then, the initial LRS state is achieved. Large resistance ratios of $10^{3}-10^{4}$ orders of magnitude are achieved in this compound too.

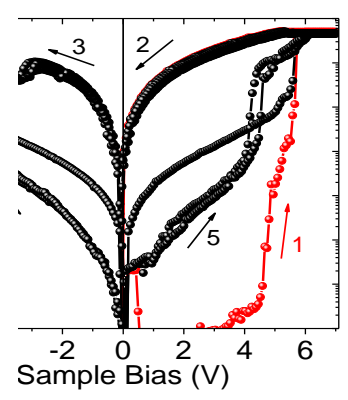

FIGURE 4.29: I-V sweep performed on a $25 \mathrm{~nm}$ LNO thin film. The first sweep is indicated in red.

As commented before, the induction of a MIT has been reported in LNO films by electric field tuning and by variations in the oxygen content $[160,297]$. These two parameters, similarly to the model exposed for LSMO and YBCO films, play a key role to explain the RS effect in these systems at it will be detailed in next sections. We can anticipate that, as in the LSMO and in the YBCO cases, the initialization step is related to oxygen-deficient regions where the composition is $\mathrm{NdNiO}_{3-x}$ and $\mathrm{LaNiO}_{3-x}$.

\section{Microstructure of RENO thin films}

The microstructure and the defects present in films might strongly influence the material properties, specially the transport properties. It was shown in chapter 2 that a large amount of Ruddlesden-Popper Faults (RPF) was detected in our RENO films. We believe that this is a stress release mechanism to stabilize the RENO phase during the growth, the system relaxes the strain by eliminating a Ni plane, which leads to a shift of half a unit cell. Both horizontal and vertical RPF are found in both LNO and NNO samples. First unit cells grow fully strained and defect free. Afterwards, RPF are generated in the film. A STEM micrograph of an NNO thin film with the RPF indicated with arrows and an explicative diagram is shown in figure 4.30 .

The thicker the film, the stronger the presence of RPF. Also, in NNO films the density of RPF is higher than in LNO samples. This is due to the higher strain in the structure induced by the smaller size of $\mathrm{Nd}$ than La. Since the metallic state in RENO systems depends on the overlapping between Ni $3 \mathrm{~d}$ and $\mathrm{O} 2 \mathrm{p}$ orbitals, the local disruption induced by the RPF might alter strongly not only the macroscopic 


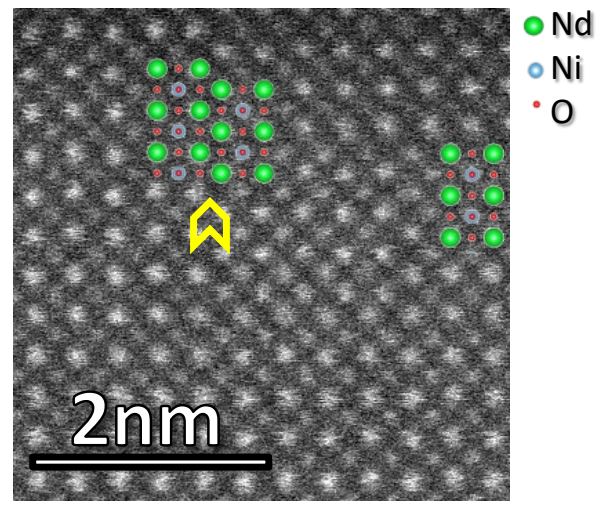

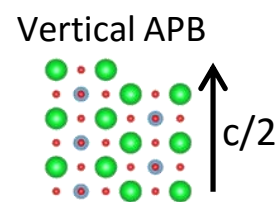

Horizontal APB

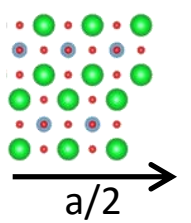

FIGURE 4.30: STEM micrograph of a NNO thin film showing RPF and its schematic diagram.

$\rho(T)$, but also the local conduction by C-AFM. Notice also that the initialization step often observed in the I-V curves suggest the presence of oxygen vacancies in our samples.

It is worth to remind that RENO compound were prepared from a stoichiometric solution in a relation 1:1 between $\mathrm{La} / \mathrm{Re}$ and $\mathrm{Ni}$. In an RPF, a plane of Ni is eliminated and therefore, the system has to place that remaining $\mathrm{Ni}$ somewhere else. In our films, the presence of $\mathrm{NiO}$ aggregates at the surface might be the answered to it. A TEM micropgrah of a NNO film where a $\mathrm{NiO}$ aggregate was detected is shown in figure 4.31. A fourier transform in the region was performed and the polycrystalline character of the $\mathrm{NiO}$ aggregates was confirmed.

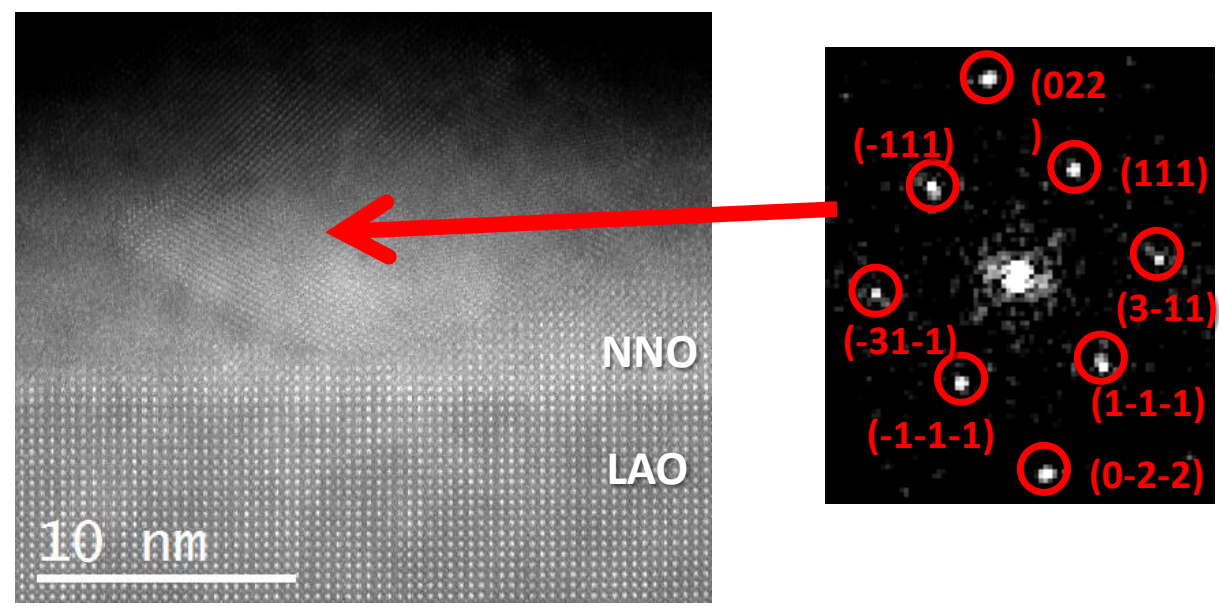

FIGURE 4.31: STEM micrograph of a NNO film grown on LAO showing a $\mathrm{NiO}$ aggregate. Fourier transform of the secondary phase.

The complex microstructure of these systems may account for the different scenarios observed in the I-V curves. We have experimentally found a variety of initial resistive states, ranging from a metallic state to highly resistive. In the former, a positive bias produces a quasi-ohmic non-hysteretic response, whereas in the latter, a HRS $\rightarrow$ LRS transition is found in the initial I-V step. Similarly to the YBCO case, this fact suggests that oxygen incorporation is induced in such oxygen-deficient regions. In addition, the structural defects ( $\mathrm{NiO}$ aggregates and the RPF) might be responsible of the variation in the resistance ratio found in the I-V sweeps. 


\section{Micrometric HR induced areas}

To study the RS properties of RENO systems at geometrically controlled micrometer scale, we have applied a set of positive and negative bias in regions of $2.5 \mu \mathrm{m} \times 2.5 \mu \mathrm{m}$ on a pristine surface of a $25 \mathrm{~nm} \mathrm{NNO}$ thin film. As depicted in the scheme in figure 4.32 (a), the voltages applied at the scan of each square were $\mathrm{V}=-$ $6 \mathrm{~V},-8 \mathrm{~V},-10 \mathrm{~V},+3 \mathrm{~V},+6 \mathrm{~V},+10 \mathrm{~V}$. After scanning the squares, a low bias $\mathrm{V}_{\text {read }}=1 \mathrm{~V}$ is used to acquire a larger scale image to readout the resistive states generated with the prior scans. Images (b) and (c) shows the topography and the resistance map of the area, respectively. Only the $+10 \mathrm{~V}$ square shows deep modifications in the topography, where a lift of $\sim 6 \mathrm{~nm}$ from the flat surface is found. In the other cases, no damage in the topography is observed. Regarding the electrical response, all squares with the applied negative bias (upper row of squares in (c)) present a higher resistive state than the pristine background. However, the application of positive bias generates different responses depending on the magnitude of the stimulus. The $+3 \mathrm{~V}$ scan generates a slightly lower resistance state than the pristine surface. This fact is more evident in the $+6 \mathrm{~V}$, where a clear lower resistive state was generated. The $+10 \mathrm{~V}$ scan, which shown a strong surface modification, has also generate a high resistance state. However, the origin of this high resistance state is different from the HRS generated upon application of a negative bias. In this case, the high positive bias applied on top of the pristine surface generates a strong change in the topography, damaging the film. Thus, probably amorphization of the material has occurred.

a)

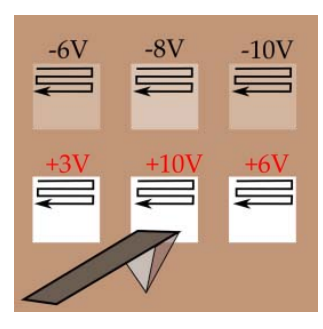

d) b)

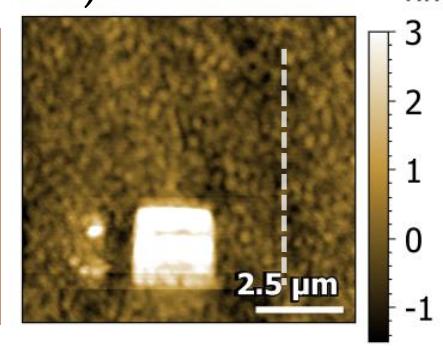

e)

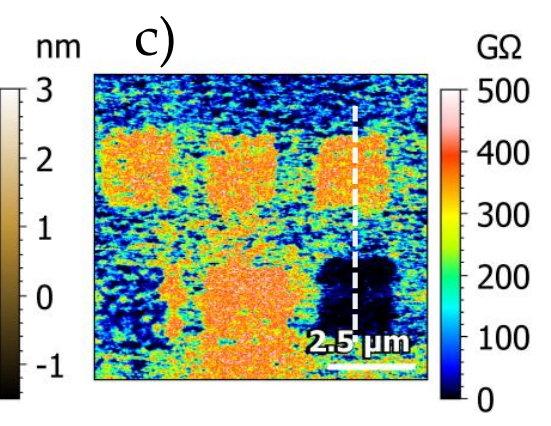

FIGURE 4.32: (a) Scheme of the applied bias on different sections of the area. (b) Topography map after applying the protocol. (c) Resistance map at 1V. White line indicate the topography (d) and resistance (e) profiles shown as dashed lines in (b) and (c), respectively.

The white line in the maps represent the profile extracted for topography (d) and resistance (e) graphs. Notice that no modification in the topogaphy profile is observed by crossing the pristine state and the HRS (-10V) - LRS (+6V) states. However, an average increase close to an order of magnitude from the pristine to the 
HRS, and a decrease of two orders of magnitude from the pristine to the LRS is found.

\section{Volume Switching in RENO thin films}

As it was shown before for the LSMO and the YBCO case, by describing the contour of a ring at high negative bias, metallic non-modified regions could be topologically isolated. In RENO systems, this experiment was performed on $6.5 \mathrm{~nm}$ sample of $\mathrm{NNO}$ grown onto LAO. On a pristine surface, two rings at high bias, namely $-6 \mathrm{~V}$ and $-10 \mathrm{~V}$ were performed. After this step, the readout of the region is performed at low bias (1V) and the obtained resistance map is shown in figure 4.33.

a)

b)

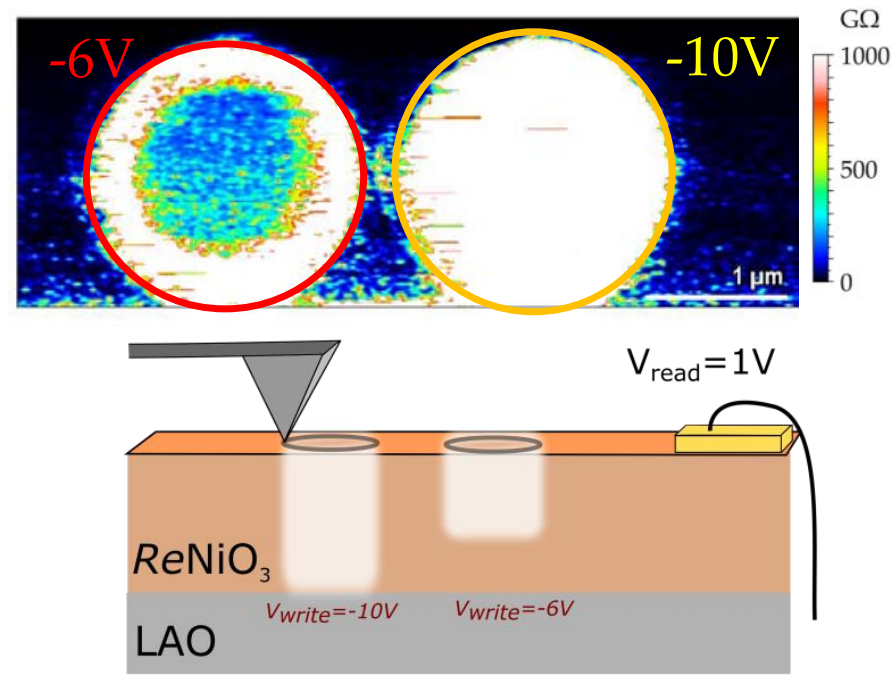

FIGURE 4.33: (a) Resistance map of a $6 \mathrm{~nm}$ NNO film after writing rings at $-6 \mathrm{~V}$ (left) and $-10 \mathrm{~V}$ (right) rings acquired at $1 \mathrm{~V}$. (b) Schematic representation of the readout process.

The ring generated at $-6 \mathrm{~V}$ presents an insulating state only at the contour of the ring. The inner part of the ring shows a metallic pristine state. The situation is described in the scheme at the bottom of the image. Some nm below the tip has been transformed into an insulating state. However, the insulating state does not reach the LAO substrate, and therefore, the current has a path to flow to the electrode during the readout scan. On the contrary, the inner part of the ring generated at $-10 \mathrm{~V}$ shows an apparent insulating state. The largest magnitude of the bias applied during the recording scan induces in this case a HRS that reaches the insulating substrate. Hence, when the tip scans the inner part of the ring during the readout, no current is collected, since the insulating wall of the ring prevents current flowing. This experiment shows the volume character of the RS in these RENO materials too, and also demonstrates that the transformed thickness can be tuned with the magnitude of the applied bias.

\section{RS characterization with micrometric $\mathrm{W}$-Au probes}

Micrometric W-Au probes $(20-50 \mu m)$ were also used to characterize the RS behaviour with a Keithley Semiconductor Analyzer. First, a $6 \mathrm{~nm}$ NNO sample was optically lithographied to define $40 \mu \mathrm{m} \times 40 \mu \mathrm{m}$ squares. Then, $60 \mathrm{~nm}$ of $\mathrm{Ag}$ were 
sputtered into the sample. A lift off process is then carried out in acetone to remove the photoresist. Two different I-V endurance cycles were performed, namely, with the W-Au tips in direct contact with the NNO surface and with the tips placed on top of the Ag electrodes. The same electric field configuration as in the rest of the thesis has been employed with the following sweep protocol. It consists of $0 \mathrm{~V} \rightarrow-10 \mathrm{~V} \rightarrow+10 \mathrm{~V} \rightarrow 0 \mathrm{~V}$ cycles several times (figure 4.34). Similarly to the RS cycles obtained with the C-AFM tip on top of the bare NNO surface, hysteresis cycles with resistance ratios between $10^{2}-10^{3}$ are obtained when the tips are placed on top of the NNO surface. A first negative sweep induces the MIT transition (step 1). The HRS is maintained in the excursion back to $0 \mathrm{~V}$ (step 2). At positive bias, the $\mathrm{OFF} \rightarrow \mathrm{ON}$ transition is induced and the system reaches a LRS (step 3). This state is maintained in the excursion back to 0V (step 4). The sweep is repeated several times showing a stabilization of both LRS and HRS, as shown in the upper right image. On the contrary, when the same protocol is performed with the tips on top of the Ag electrodes, the RS effect is mostly suppressed and tiny ratios of $\sim 1,2-1,3$ times distinguishes the LRS and the HRS.

a)
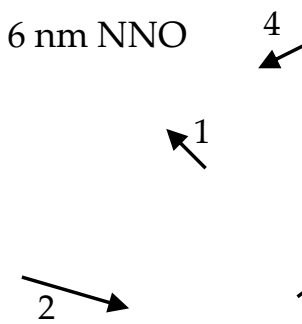

c)

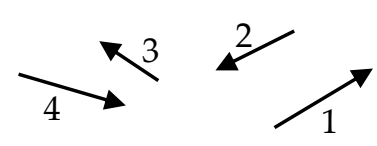

$\mathrm{Ag} / \mathrm{NNO}$ b)

$\mathrm{V}_{\text {read }}=0.5 \mathrm{~V}$

d)

\begin{abstract}
FIGURE 4.34: I-V sweeps performed with W-Au macroscopic of a 6 nm NNO thin tips. (a) on top of the bare surface film. (b) On top of Ag electrodes (c). (b) and (d) show the resistance values obtained at $0.5 \mathrm{~V}$ from (a) and (c) respectively.
\end{abstract}

$\mathrm{V}_{\text {read }}=0.5 \mathrm{~V}$

For clarification, the cumulative frequency counts for both the LRS and the HRS of the cycling performed in 4.34 (a) is shown in figure 4.35. Both states are separated by approximately two orders of magnitude. In addition, both states present a similar deviation of around one order of magnitude.

It should be taken into account that the larger size of the W-Au tips probably produces a modification of the field distribution, so RS parameters may change accordingly. The lack of knowledge about the exact contact area, contact resistance and particular electric field distribution prevents a reliable comparison between these measurements and the C-AFM ones. Further experiments with highly contrable geometry would need to be performed to address this issue. 
FIGURE 4.35: Cumulative plot of the HRS and LRS in the NNO measured with W-Au microprobes.

In conclusion, the nanoscopic behaviour in RENO systems have been successfully demonstrated at a larger scale with micrometric probes and show great potential for further device integration and testing. Further studies are required to properly address the RS parameteres, such as endurance tests, retention times, switching dynamics in more realistic architectures (for instance, a $\mathrm{CeO}_{2} / \mathrm{RENO}$ bilayer).

\section{Resistive Switching mechanism in RENO systems}

The experimental evidences showed along this chapter suggest that the same mechanism that for YBCO and LSMO thin films is the responsible of the RS in RENO compounds. When the tip is placed in contact with the surface and an intense downwards electric field is applied, two simultaneous effects occur. Charge injection, which decreases the hole carrier density of the system and $\mathrm{O}_{\mathrm{RENO}}^{2-}$ species are driven towards the surface. These anions react with adsorbate $\mathrm{O}^{2-}$ species to form an oxygen molecule. In this way, the system balances the charge injection by creating oxygen vacancies. These vacancies diffuses into the bulk along the electric field direction. A valence change in the Ni oxidation state $\left(\mathrm{Ni}^{3+} \rightarrow \mathrm{Ni}^{2+}\right)$ is induced. This physical mechanism is stimulated by the MIT of these systems. To recover the LRS initial state, electric field polarity should be reversed and the opposite situation occurs. Adsorbate species at the surface are driven into the material occupying the place of an oxygen vacancy. This induces the oxidation of $\mathrm{Ni}$ and the initial valence is restored. Eventually, the LRS is achieved. With micrometric W-Au probes, the same behaviour is found when the tips are placed on the surface. However, the presence of a barrier for oxygen exchange with ambient as the Ag electrodes suppresses the RS effect. The microstructural landscape, including $\mathrm{NiO}$ aggregates and Ruddlesden-Popper Faults, have probably a strong influence in the RS properties, which results in a large variety of I-V curve characterization.

\subsubsection{RS characterization in $\mathrm{RENiO}_{3}$ grown by RF-Sputtering}

To further investigate the nature of the RS effect in these RENO systems, we have also analysed RENO films grown by physical methods. By the end of this thesis, we have started a collaboration with Dr. Marta Gibert, Mrs. Sara Catalano and Prof. Jean-Marc Triscone from the University of Geneva (Switzerland). They have provided us with three RENO samples grown by RF-Sputtering. This technique is widely used in thin film growth. It is an out-of-equilibrium process, and therefore, a different defect landscape than the CSD approach is expected. For more 
details about the growth of these films, the reader is referred to the publications of Triscone's group [78, 100, 297].

Three samples with different $\mathrm{T}_{\mathrm{MIT}}$ have been analysed, namely a $\mathrm{LaNiO}_{3}$ and $\mathrm{NdNiO}_{3}$ grown on $\mathrm{LAO}$ substrates (LNO/LAO and NNO/LAO, respectively), and $\mathrm{NdNiO}_{3}$ grown on $\mathrm{NdGaO}_{3}(\mathrm{NNO} / \mathrm{NGO})$ substrate. The three samples present a very similar thickness of 12 u.c. $(\sim 6 \mathrm{~nm})$. As it was discussed in chapters 1 and 2 , the selection of a particular substrate allows to tune the Ni-O-Ni angle through strain effects, which has a strong impact on the physical properties of the system [78]. Besides lattice mismatch, substrate symmetry and orientation can also induce extra distortions and modify the tilting of the oxygen octahedron $[78,300]$. The in-plane strain of the films used in this section is shown in table 4.1.

\begin{tabular}{|c|c|c|c|}
\hline In-plane strain Sample & $\mathrm{LNO} / \mathrm{LAO}$ & $\mathrm{NNO} / \mathrm{LAO}$ & $\mathrm{NNO} / \mathrm{NGO}$ \\
\hline$\epsilon(\%)$ & -1.3 & -0.59 & +1.1 \\
\hline
\end{tabular}

TABLE 4.1: Mismatch between substrates and RENO films grown by RF-sputtering.

The MIT of these three systems have been analysed by means of 4-point measurements in Van der Pauw[157] configuration as a function of temperature. The resistivity curves are shown in figure 4.36. The LNO sample does not present MIT, as stated for the CSD case and for the bulk in chapters 1 and 2. For both NNO samples, a sharp metal-insulator transition occurs at $\mathrm{T}_{\mathrm{MIT}} \approx 70 \mathrm{~K}$ and $170 \mathrm{~K}$ when grown on LAO and NGO, respectively. Compared to the performance of our CSD samples, the metal-insulator transition of these NNO sputtered films is more abrupt and with a larger change in the resistance $\left(10^{2}-10^{3}\right)$ in the insulating state than for the CSD case. This is an indication of a lower density of microstructural defects in the films grown by sputtering, since the strain imposed in our films was the same for the LNO and NNO cases grown onto LAO. Here, we also present the NNO/NGO, which presents a tensile strain of $\sim 1.1 \%$. This in-plane strain drives the $\mathrm{T}_{M I T}$ towards higher temperatures, closer to the bulk value.

FIGURE 4.36: Resistivity as a function of temperature for $6 \mathrm{~nm}$ RENO samples grown by RF-Sputtering.

\section{Electrical characterization by I-V sweeps}

As exposed along this thesis, the C-AFM measurements were conducted in a toptop configuration with the tip grounded (see chapter 2). Therefore, a negative bias 
creates an intense electric field pointing downwards at the tip apex, whereas a positive bias generates an electric field pointing upwards. In this section, I-V sweeps showing bipolar RS in the three RENO sputtered films are presented. In the three cases, the same protocol was applied to acquire the data shown in figure 4.37, namely $0 \rightarrow-10 \mathrm{~V} \rightarrow 0 \mathrm{~V} \rightarrow+10 \mathrm{~V} \rightarrow 0 \mathrm{~V}$. As in the canonical examples of the CSD films, the first sweep, indicated with a red arrow, starts towards negative bias. In the three cases, the transition from the pristine metallic LRS to a HRS occurs. This $\mathrm{ON} \rightarrow \mathrm{OFF}$ transition presents a correlation with the MIT previously shown in the resistivity plot (figure 4.36). The LNO case shows a smoother transition to the OFF state than both NNO samples, which present a sharper transition to a deeper HRS. For the NNO/NGO sample, which show the highest transition temperature of the set of samples ( $\mathrm{T}_{\mathrm{MIT}} \approx 170 \mathrm{~K}$ ), the LRS $\rightarrow$ HRS transition is specially sharp with a deep change in the resistance state. However, these I-V are rather scattered and show less reproducibility than the films grown on LAO.

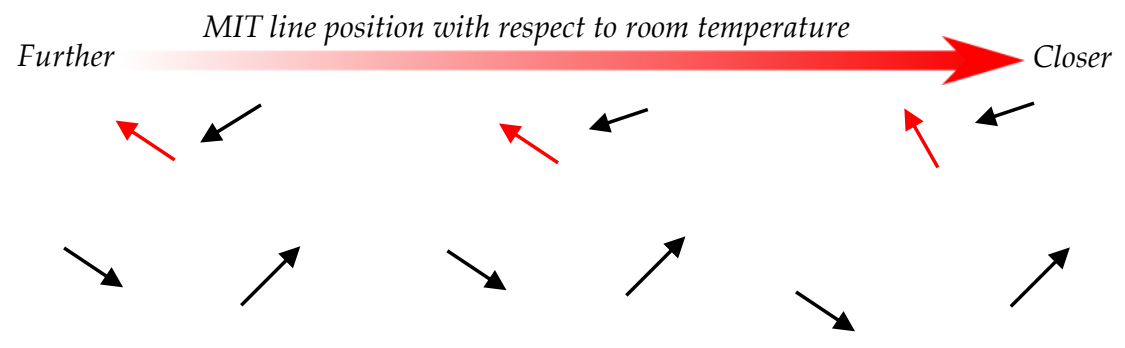

FIGURE 4.37: I-V sweeps performed by C-AFM. The arrows indicate the direction of the sweep (first sweep in red). (a) LNO/LAO. (b) $\mathrm{NNO} / \mathrm{LAO}$. (c) NNO/NGO.

The second part of the sweep corresponds to the $-10 \mathrm{~V} \rightarrow+10 \mathrm{~V}$ branch, in which the HRS is kept until a transition from the OFF to the ON state occurs at positive bias. The voltages at which this HRS $\rightarrow$ LRS takes place can also be correlate with the MIT of the system and will be carefully analysed in next section, together with the resistance ratio. The last branch of the I-V sweep corresponds to the excursion back to $0 \mathrm{~V}$, where the LRS is kept.

In this way, we have demonstrated the excellent performance of RENO sputtered films for RRAM applications, specially the LNO/LAO and NNO/LAO films, showing the highest resistance ratios and reproducibility.

\section{Discussion}

For comparison, three I-V sweeps for each sample have been plotted together in figure 4.38. The selected I-V characteristics show representative examples obtained in each of the films. The main parameters under evaluation in this section are the transition voltages (grey squares in the figure) and the resistance ratio $\mathrm{R}_{\mathrm{OFF}} / \mathrm{R}_{\mathrm{ON}}$. This ratio will be evaluated at $\mathrm{V}_{\text {bias }}=1 \mathrm{~V}$ as indicated with the dashed line in the figure.

We have extracted the values for the transition voltages $\left(\mathrm{V}_{\mathrm{LRS} \rightarrow} \mathrm{HRS}\right)$ and $\mathrm{V}_{\mathrm{HRS} \rightarrow} \mathrm{LRS}$ ) and the resistance values at $1 \mathrm{~V}$ in the LRS and the HRS for more than $50 \mathrm{I}-\mathrm{V}$ curves per sample, at random locations on the surface. 

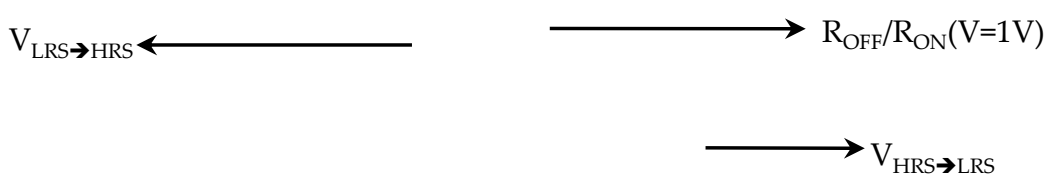

FIGURE 4.38: Comparison of I-V sweeps for the three RENO samples. The parameters to be analysed are indicated by grey squares (transition voltages) and dashed line (resistance ratio).

With this set of data, we have performed and statistical analysis and extracted the cumulative frequency counts. In figure 4.39 (a), the cumulative frequency for the threshold voltages (LRS $\rightarrow$ HRS at negative bias and HRS $\rightarrow$ LRS at positive bias) are shown. A clear correlation between the $\mathrm{ON} \rightarrow \mathrm{OFF}$ transition and the MIT of the films is observed. In the three cases narrow threshold voltage distributions are obtained. The higher values are found for the $\mathrm{LNO} / \mathrm{LAO}$ sample, which do not show any $\mathrm{T}_{M I T}$ down to $5 \mathrm{~K}$. In contrast, the lowest values correspond to the NNO/NGO sample, which present the highest $\mathrm{T}_{\mathrm{MIT}}$. An intermediate situation occurs for the $\mathrm{NNO} / \mathrm{LAO}$ sample, which also presents a $\mathrm{T}_{M I T}$ but at lower temperatures. At the positive branch, in contrast, the $\mathrm{LNO} / \mathrm{LAO}$ and NNO/LAO sample presents very similar transition threshold voltages with narrow distributions. The NNO/NGO sample has a wider distribution with lower transition voltages.

a)

b)

FIGURE 4.39: Cumulative frequency plots for (a) the transition voltages and (b) the resistance values of the LRS and the HRS.

The cumulative frequency distribution of the LRS and the HRS for the three samples is shown in the figure 4.39 (b). As expected from the resistivity plot in figure 4.36, both samples grown on LAO (NNO and LNO) present a very similar LRS $10^{5} \Omega$ with a narrow distribution. The LRS of the NNO/NGO sample shows a slightly higher resistance value with a wider distribution $\left(10^{5}-10^{6} \Omega\right)$. A clear correlation between the MIT of the systems and the generated HRS is observed. The highest HRS $\left(\sim 10^{9}-10^{11} \Omega\right)$ corresponds to the NNO/NGO sample, with the MIT closer to room temperature. The lowest HRS is achieved in the LNO sample, whose MIT line lies below $5 \mathrm{~K}$, with resistance values in the range of $10^{7}-10^{9} \Omega$. An intermediate situation is observed for the $\mathrm{NNO} / \mathrm{LAO}$ sample. 


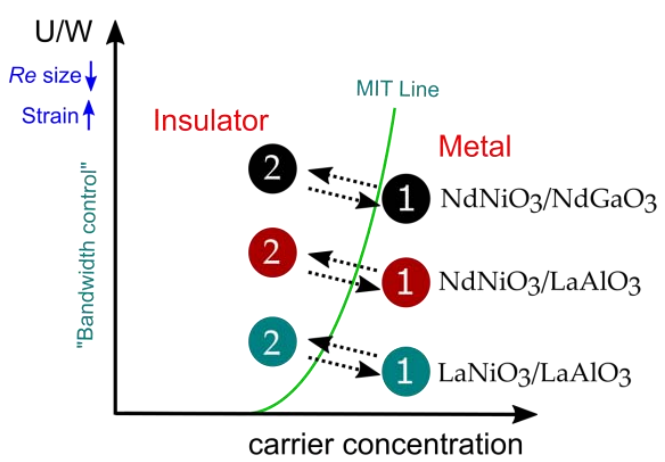

FIGURE 4.40: Bandwidth-control versus band filling control in RENO systems. By changing the size of the RE cation or by strain [78], the bandwidth of the systems is varied and therefore, the MIT is tuned. The green line represents the MIT line.

Therefore, by engineering the material growth we can tune the MIT of these RENO systems, modifying the bandwidth, and consequently the RS parameters and performance. By changing the rare-earth cation size or by applying biaxial strain [78], the initial state of the system can be tuned as shown in the schematic diagram in figure 4.40. The closer the initial state to the MIT line, the lower operating voltages required for the MIT-RS transitions and the higher resistance ratios are obtained. Further electrical characterization should be performed to address repeatability studies, specially on the NNO/NGO sample, where wider distributions are obtained,specially for the HRS.

The RS induced MIT observed in these RENO might be explained in the same way as for LSMO, YBCO and CSD-RENO samples (discussed in previous section). The electric-field-induced charge injection to the system and the concomitant oxygen exchange with ambient, which generates oxygen vacancies in the film. This process produces a valence change in the Ni cations bringing the system closer the MIT (or inducing a proper MIT) with a consequent colossal change in the resistance state, thus these systems are specially suitable as memristive elements.

\subsubsection{Conclusions}

To sum up, the resistive switching properties of RENO thin films have been thoroughly investigated at the nanoscale by means of C-AFM. The RENO thin films have been synthesized by two different methodologies, namely Chemical Solution Deposition and RF-Sputtering. The former is a thermodynamic equilibrium process whereas the latter is an out-of equilibrium process, which leads to different microstructure of both kind of films. In both cases, bipolar RS characteristics are obtained with large resistance ratios of $10^{3}-10^{5}$ between the pristine LRS initial state and the generated HRS. A volume RS behaviour is also demonstrated for these films. In the particular case of our CSD samples, we detected the presence of large amounts of defects (Ruddlesden-Popper Faults (RPF) and oxygen vacancies) and secondary phases ( $\mathrm{NiO}$ aggregates). We have found a correlation between these defects and the I-V RS characteristics obtained by C-AFM. In particular, oxygen vacancies might account for a non-metallic initial state (observed in many points) that requires an initialization step by the application of a large positive bias. The presence of $\mathrm{NiO}$ and $\mathrm{RPF}$, where a Ni plane is eliminated, might account for the 
variability observed in the generated HRS, and therefore, a random distribution of $\mathrm{R}_{\mathrm{OFF}} / \mathrm{R}_{\mathrm{ON}}$ ratios. These inhomogeneities are also confirmed by micrometric scans with the AFM tip at positive and negative bias. In the case of the films grown by sputtering, a lower density of defects is expected from the sharp MIT transitions observed in the resistivity plots $\rho(T)$. The RS characteristics of $6 \mathrm{~nm}$ with different strain films have been carefully analysed by C-AFM. In particular, we have applied statistical methods to evaluate the transition voltages and the resistance values of the LRS and the HRS. A fine correlation of the RS parameters with the MIT initial position of the films (mainly determined by the RE size cation and the biaxial strain) is observed. The closer the MIT to the operating temperature (room temperature), the lower the threshold voltages and the larger the resistance ratios obtained. Resistance ratios of $10^{4}-10^{5}$ are generated for the NNO samples, while ratios of $10^{3}$ are observed in the $\mathrm{LNO} / \mathrm{LAO}$ case.

Good MIT-RS performances of the RENO systems grown by two different methodologies are thus presented and correlated to the intrinsic MIT properties of the films. These strongly correlated systems appear to be therefore suitable candidates for R-RAM applications. Further studies are now required to integrate these films in more realistic device-like architectures to evaluate accurately several performance parameters such as retention times, switching speeds, endurance tests. 



\section{Chapter 5}

\section{General conclusions}

In this thesis, we have investigated bipolar resistive switching phenomena that can be induced in three different metallic mixed-valence-mixed-conductors perovskite oxides upon the application of an electric field: $\mathrm{La}_{1-x} \mathrm{Sr}_{x} \mathrm{MnO}_{3}$ (LSMO), $\mathrm{YBa}_{2} \mathrm{Cu}_{3} \mathrm{O}_{7-\delta}(\mathrm{YBCO})$ and $\mathrm{ReNiO}_{3}$ (RNO). Despite the different structural and electronic characteristics of the systems studied, a similar phenomenology is found. RS in these compounds can be explained in terms of a valence change of the transition metal induced by the exchange of oxygen with the ambient atmosphere driven by the electric field. This oxygen exchange induces simultaneously a variation of the carrier density which through the MIT of these systems induces a volumetric resistive switching phenomena.

$\mathrm{La}_{1-x} \mathrm{Sr}_{x} \mathrm{MnO}_{3}$ and $R e \mathrm{NiO}_{3}$ films were grown on insulating substrates (STO and LAO, respectively) by low cost route of Chemical Solution Deposition (CSD). Superconducting YBCO samples were grown by Pulsed Laser Deposition (PLD) on insulating STO substrates. High-quality epitaxial films with smooth surfaces and low roughness are obtained for the three compounds. In addition, $\mathrm{CeO}_{2}$ thin layers were ex-situ deposited by Atomic Layer Deposition (ALD) on top of YBCO and LSMO films. The physical properties of the underlying metallic perovskite remains unaffected after this deposition process.

In the $\mathrm{La}_{1-x} \mathrm{Sr}_{x} \mathrm{MnO}_{3}$ case, the bipolar RS characteristics with high resistance ratios of $10^{3}-10^{4}$ were linked to the MIT of these systems, which can be tuned with the Sr content of the film modifying the RS parameters accordingly. We have demonstrated that the switching might occur even through the full thickness of the film if high enough bias voltage are applied, illustrated by C-AFM experiments where a HRS ring was drawn, enabling the isolation non-modified pristine regions. Accordingly, we have shown that it is possible to achieve multilevel resistance states by proper tuning of the applied bias. We have proposed that the MIT is the primary responsible of the strong character of the RS effect in LSMO thin films. Upon the application of the electric field, the simultaneous decrease in the number of hole carriers and oxygen anion excorporation induces a valence change in the Mn metal cations, which eventually drives the metallic system into an insulating state. The reverse process is favoured when the electric field is reversed.

The proposed oxygen exchange with ambient was proved by means of atmosphere dependent measurements. The decrease in the adsorbate surface coverage at lower $\mathrm{O}_{2}$ pressures produces that only a reduced number of ions are exchanged and therefore, a lower HRS is achieved under such conditions. In contrast, the experiments carried out in $\mathrm{Ag} / \mathrm{CeO}_{2} / \mathrm{LSMO}$ heterostructures have revealed that the exchange does not occur through the ambient, but through a direct ionic exchange between 
the $\mathrm{LSMO}$ and the $\mathrm{CeO}_{2}$ layers. Highly symmetric sweeps with sharp transitions are found in this bilayer heterostructures, confirming the properties of $\mathrm{CeO}_{2}$ as an oxygen vacancy reservoir.

Moreover, the electronic properties of HRS regions were studied by tunnelling spectroscopy and 4-points transport measurements, which provide strong evidences supporting the strong-correlation effects scenario behind the RS effect. Specifically, we have shown that the MIT can be fully crossed at RT through an increase of resistance by two orders of magnitude and a concomitant shift of the $\mathrm{T}_{\mathrm{MIT}}$ to lower temperatures.

Finally, a proof-of-principle three-terminal (3T) configuration is proposed in which the lateral conduction of a LSMO bridge, between source and drain, is modified by applying RS in a region located at the middle of the conduction bridge (gate). Reversible resistance changes of two orders of magnitude are found and a volume resistive switching is also demonstrated at the micrometric scale, where the full thickness of the film below the W-Au tip has been transformed into the HRS.

The RS properties of pristine $\mathrm{YBa}_{2} \mathrm{Cu}_{3} \mathrm{O}_{7-\delta}$ and $\mathrm{CeO}_{2} / \mathrm{YBa}_{2} \mathrm{Cu}_{3} \mathrm{O}_{7-\delta}$ thin films were also investigated by C-AFM. In both cases, bipolar resistive switching characteristics are observed. However, different I-V behaviours are found, and each of them can be interpreted in terms of random variations of the oxygen content at the nanoscale. An initial positive bias sweep might be required to induce first oxygen incorporation into the initial partially (or totally) deoxygenated region showing a higher resistive state than the expected pristine LRS state with resistance ratios of $10^{2}-10^{3}$.

Surface degradation has been observed in bare YBCO patterned bridges after inducing the RS upon air exposure. This degradation has been avoided by using 3-10 nm $\mathrm{CeO}_{2}$ capping layer. HRS arrays of different geometries have been induced in $\mathrm{YBCO}$ and $\mathrm{CeO}_{2} / \mathrm{YBCO}$ patterned bridges to analyse the influence of the HRS regions on the superconducting transport properties of the bridge. We have assessed the influence of the micro- and nanometric HRS domains on the superconducting properties of the film. With this approach, we have demonstrated the critical current density dependency on the applied magnetic field was modified in the presence of HRS dots array.

In the $\mathrm{RNiO}_{3}$ case, additionally to the CSD route for the growth of epitaxial films, we have also investigated the RS capabilities of RF-sputtered films. Samples grown by both methodologies show bipolar RS characteristics with large resistance ratios of $10^{3}-10^{5}$ between the pristine LRS initial state and the generated HRS. We have analysed the RS parameters for both growth techniques, as well as their possible relation with the microstructural defects, showing that the electronic transport might be strongly influenced by them.

In the particular case of our CSD-RNO films, we have suggested a correlation between the presence of secondary phases (NiO aggregates), Ruddlesden-Popper Faults (RPF) and oxygen vacancies with the RS characteristics of the films. Oxygen vacancies might account for a non-metallic initial state that requires an initialization step by the application of a large positive bias. The presence of $\mathrm{NiO}$ and RPF might account for the variability observed in the generated HRS, which produces a random distribution of $\mathrm{R}_{\mathrm{OFF}} / \mathrm{R}_{\mathrm{ON}}$ ratios. 
In the case of the sputtered films, a lower density of defects is expected from the sharp MIT transitions observed in the resistivity plots. The RS characteristics of the films have been carefully analysed by C-AFM through statistical analysis to evaluate the threshold transition voltages and the resistance values of the LRS and the HRS. A fine correlation of the RS parameters with the initial position MIT of the films, mainly determined by the Re size cation and the biaxial strain, is observed. The closer the MIT to the operating temperature (room temperature), the lower the threshold voltages and the larger the resistance ratios obtained. Resistance ratios of $10^{4}-10^{5}$ are generated for the NNO samples, while a ratio of $10^{3}$ is observed in the $\mathrm{LNO} / \mathrm{LAO}$ case.

In summary, we have verified that strongly correlated metallic perovskite oxides are a unique class of materials very promising for RS applications due to its intrinsic MIT properties that boosts a robust volumetric resistive switching effect. Still several parameters should be properly tested and tuned for applications needs (like lower threshold voltages, endurance, switching speed, retention studies). This thesis settles down the framework to understand the RS effect in these strongly correlated pervoskites, which could eventually lead to a new generation of devices exploiting the intrinsic MIT of these systems. 



\section{Appendix A}

\section{Experimental techniques}

In this section we provide an overview of the working principles of the experimental techniques used for the analysis of the structural and physical properties of our samples. It includes Scanning Electron Microscopy (SEM), X-Ray Diffraction (XRD), Physical Properties Measurement system (PPMS) and Superconducting Quantum Interference Device (SQUID). Some of these techniques were operated with the help of the technical staff of the scientific-technical services of ICMABCSIC.

\section{A.1 Field Emission Scanning Electron Microscopy}

In the basic setup of a Scanning Electron Microscopy (SEM), the sample is analysed using an electron beam with an energy in the range of $\mathrm{keV}$ of $1-20 \mathrm{keV}$. An SEM typical setup is depicted in figure A.1. This is an extremely useful tool to reveal information about the specimen's topography, grain size, porosity, particle distribution and inhomogeneities. In addition, it can provide compositional information from the average atomic number, as well as surface potential distribution, magnetic domains or crystal orientation and defects. The main drawback of the basic SEM setup is that the samples must be electrical conductors. Insulating samples can be studied if they are properly coated with a thin conducting film. However, this issue is overcome in the Field Emission SEM (FESEM), which provides a significant improvement in resolution, as well as the possibility to work with non-conducting samples. In short, FESEM provide narrower beams, resulting in an enhancement of the spatial resolution (down to $5 \mathrm{~nm}$ ), and a reduction in sample charging and damage. The interaction of the primary electrons from the source with the sample results in different information that can be obtained through backscattered electrons, secondary electrons and characteristic X-rays. Secondary electrons (energy $<1-50 \mathrm{eV}$ ) provide information about surface features. Backscattered electrons are the ones with a strong interaction with atom nuclei (energy $>50 \mathrm{eV}$ ), which provide compositional information (z-contrast). Furthermore, the primary electron beam generates characteristic $X$-rays which can be used to give more accurate data about composition, also known as Energy-dispersive X-ray spectroscopy (EDX). FESEM images were made using a FEI QUANTA 200 (FEG-ESEM). 


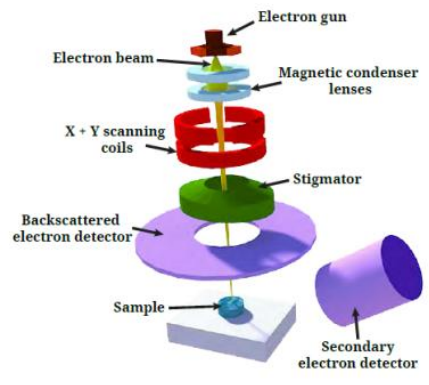

FIGURE A.1: Diagram describing the general set-up of a SEM.

\section{A.2 X-ray diffraction}

X-rays are electromagnetic waves with wavelengths in the same order as interatomic distances in solids $(0.1-100 \AA)$, thus allowing their interaction with the electronic arrangement of atoms in a solid, and giving information about its internal distribution. When X-rays hit a crystalline sample, they are scattered due to the interaction with the electrons surrounding the atoms. If the scattered X-rays fulfil the Bragg condition

$$
n \lambda=2 d_{h, k, l} \sin (\theta)
$$

where $\lambda$ is the wavelength, $d$ is the distance between each adjacent crystal plane ( $d$ spacing), and $\theta$ is the Bragg angle at which one observes a diffraction, a constructive interference will be produced and a diffraction peak will be detected. Figure A.2 (a) shows an schematic representation of X-ray measurement and the Bragg's law. A diffraction pattern can be thus measured upon variations in the incidence angle of X-rays. The obtained diffraction pattern is a characteristic fingerprint of a particular material. In this sense, the position and intensity of of each Bragg peak in a diffraction pattern essentially depends on the characteristics of the unit cell (symmetry and size), the particular arrangement of the atomic species within it and on the radiation employed. In the specific case of highly oriented specimens such as single crystals or epitaxial thin films, one can only find specific families of $h k l$ planes satisfying the diffraction conditions. Modifying the relative position between detector and sample, the specific diffraction conditions can be achieved (figure A.2 (b). In this thesis, we have performed one dimensional $\theta-2 \theta$ scans, which gives information about the crystallographic orientation of our films. These measurements were carried out in a Rigaku Rotaflex RU-200BV diffractometer with a $C u_{K_{\alpha}}=1.5418 \AA$ located at ICMAB-CSIC facilities. The typical step size employed in the measurements was $0.02^{\circ}$.

a)

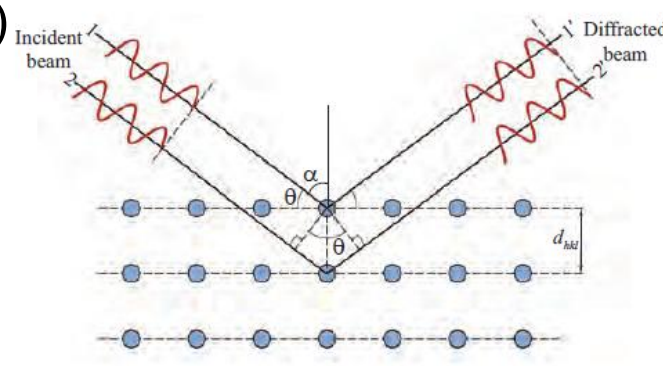

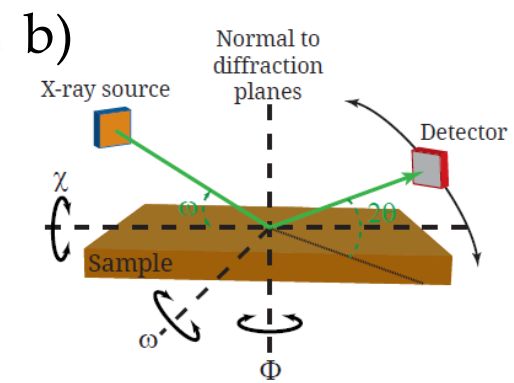

FIGURE A.2: (a) Schematic description of the Bragg's diffraction law.

(b) General set up and angle nomenclature in XRD measurements. 


\section{A.3 Physical Properties Measurement System}

The electric transport measurements as a function of temperature and field show in this thesis have been performed using a Physical Properties Measurements System (PPMS) from Quantum Design, located at the ICMAB-CSIC. The setup is equipped with 9T (resolution of $0.1 \mathrm{mT}$ ) superconducting magnet and a helium cryostat working precisely in the temperature range of $1.8 \mathrm{~K}-400 \mathrm{~K}$. The transport properties are sensed with a nano-voltmeter and a AC/DC current source $(1 \mu A-A$ with a resolution of $0.1 \mu A$ ). Vacuum grease is used to mount the sample to the holder in order to provide a good electrical insulation and a good thermal contact. A thermometer is located right below the sample holder space to monitor the sample temperature.

\section{A.4 Superconducting Quantum Interference Device}

A superconducting quantum interference device (SQUID) is a very sensitive magnetometer allowing the measurement of incredibly small magnetic fields. The SQUID itself consists of a superconducting loop with one or two weak links (Josephson junctions) in it. In short, they generate an output voltage signal, which is a periodic function of the magnetic flux through the loop, a geometry known as DC-SQUID. The sample is mounted inside this coil and an uniform dc-magnetic field is applied. Once the sample is magnetized, it is moved through a set of pickup coils. The sample displacement induces a current in the detection system which is proportional to the variation of the magnetic flux. This signal is carried to the SQUID sensor, where it is amplified as a voltage signal. In essence, this generated voltage is proportional to the magnetic moment of the specimen. The described setup is mounted in a helium cryostat to enable temperature dependent measurements. Magnetization curves of LSMO and YBCO thin films were measured at ICMAB facilities using a commercial MPMS XL-7T SQUID DC-magnetometer (Quantum Design) equipped with a 7T superconducting coil and a helium cryostat working in the $1.8 \mathrm{~K}-400 \mathrm{~K}$. 



\section{Bibliography}

[1] IDC. Where in the world is the storage. Byte Density Across the Globe. http: //www.idc.com/downloads/where_is_storage_infographic_ 243338 .pdf. [Online; accessed 30-November-2016]. 2015.

[2] G. E. Moore. "Cramming more components onto integrated circuits". In: Electronics (1965), pp. 114-117. DOI: 10.1109 / JPROC .1998.658762.

[3] C. Y. Lu, K. Y. Hsieh, and R. Liu. "Future challenges of flash memory technologies". In: Microelectronic Engineering 86 (3) (2009), pp. 283-286. DOI: $10.1016 / j$.mee.2008.08.007.

[4] International Roadmap for Semiconductors. Beyond CMOS. http://www. semiconductors. org / clientuploads / Research_Technology / ITRS/2015/6_2015_20ITRS_202.0_20Beyond_20CMOS.pdf. [Online; accessed 30-November-2016]. 2015.

[5] Yoshihisa Fujisaki. "Review of emerging new solid-state non-volatile $\mathrm{m}$ memories". In: Jap. J. Appl. Phys. 52 (2013), p. 40001. DOI: 10.7567 / J JAP . 52.040001 .

[6] J. S. Meena, S. M. Sze, U. Chand, and T.-Y. Tseng. “Overview of emerging nonvolatile memory technologies." In: Nanoscale research letters 9 (1) (2014), p. 526. DOI: $10.1186 / 1556-276 \mathrm{X}-9-526$.

[7] S. Parkin, X. Jiang, C. Kaiser, A. Panchula, K. Roche, and M. Samant. Magnetically engineered spintronic sensors and memory. 2003. DOI: $10.1109 /$ JPROC.2003.811807.

[8] J.-M. Hu, Z. Li, L.-Q. Chen, and C.-W. Nan. "High-density magnetoresistive random access memory operating at ultralow voltage at room temperature". In: Nature Communications 2 (2011), p. 553. DOI: 10.1038 / ncomms 1564.

[9] M. Wuttig and N. Yamada. "Phase-change materials for rewriteable data storage". In: Nature Materials 6 (11) (2007), pp. 824-832. DOI: 10.1038 / nmat2009.

[10] S. Raoux, G. W. Burr, M. J. Breitwisch, C. T. Rettner, Y.-C. Chen, R. M. Shelby, M. Salinga, D. Krebs, S.-H. Chen, H.-L. Lung, and C. H. Lam. "Phase-change random access memory: A scalable technology". In: IBM Journal of Research and Development 52 (4-5) (2008), pp. 465-479.

[11] O. Auciello, J. F. Scott, and R. Ramesh. "The Physics of Ferroelectric Memories". In: Physics Today 51 (7) (1998). DOI: http: / / dx . doi . org/ 10 . $1063 / 1.882324$.

[12] S. Nakamura and H. Ishiwara. "Current status of ferroelectric RandomAccess memory". In: MRS Bulletin 29 (11) (2004), pp. 823-828. DOI: https: //doi.org/10.1557/mrs2004.235.

[13] A. Sawa. "Resistive switching in transition metal oxides". In: Materials Today 11 (6) (2008), pp. 28-36. DOI: $10.1016 /$ S1369-7021 (08) 70119-6.

[14] R. Waser and M. Aono. "Nanoionics-based resistive switching memories." In: Nature materials 6 (11) (2007), pp. 833-40. DOI: 10.1038 / nmat2 023.

[15] R. Waser, R. Dittmann, G. Staikov, and K. Szot. "Redox-Based Resistive Switching Memories - Nanoionic Mechanisms, Prospects, and Challenges". 
In: Advanced Materials 21 (25-26) (2009), pp. 2632-2663. DOI: $10.1002 /$ adma. 200900375.

[16] J. J. Yang, D. B. Strukov, and D. R. Stewart. "Memristive devices for computing." In: Nature nanotechnology 8 (1) (2013), pp. 13-24. DOI: $10.1038 /$ nnano.2012.240.

[17] D.H. Kwon, K. M. Kim, J. H. Jang, J. M. Jeon, M. H. Lee, G. H. Kim, X.-S. Li, G.-S. Park, B. Lee, S. Han, M. Kim, and C. S. Hwang. "Atomic structure of conducting nanofilaments in $\mathrm{TiO}_{2}$ resistive switching memory." In: Nature nanotechnology 5 (2) (2010), pp. 148-53. DOI: 10.1038 / nnano.2009. 456.

[18] J. B. Yun, S. Kim, S. Seo, M. J. Lee, D. C. Kim, S. E. Ahn, Y. Park, J. Kim, and H. Shin. "Random and localized resistive switching observation in $\mathrm{Pt} / \mathrm{NiO} / \mathrm{Pt}$ ". In: Physica Status Solidi - Rapid Research Letters 1 (6) (2007), pp. 280-282. DOI: 10.1002/pssr.200701205.

[19] G.-S. Park, Y. B. Kim, S. Y. Park, X. Shu Li, Sung Heo, Myoung-Jae Lee, Man Chang, Ji H. Kwon, M. Kim, U.-I. Chung, R. Dittmann, R. Waser, and K. Kim. "In situ observation of filamentary conducting channels in an asymmetric $\mathrm{Ta}_{2} \mathrm{O}_{5-x} / \mathrm{TaO}_{2-x}$ bilayer structure." In: Nature communications 4 (2013), p. 2382. DOI: $10.1038 /$ ncomms 3382 .

[20] A. Herpers, C. Lenser, C. Park, F. Offi, F. Borgatti, G. Panaccione, S. Menzel, R. Waser, and R. Dittmann. "Spectroscopic proof of the correlation between redox-state and charge-carrier transport at the interface of resistively switching Ti/PCMO devices". In: Advanced Materials 26 (17) (2014), pp. 2730-2735. DOI: 10.1002 / adma. 201304054.

[21] H. Lee, S. Choi, H. Park, and M. Rozenberg. "A new route to the Mott-Hubbard metal-insulator transition: Strong correlations effects in $\mathrm{Pr}_{0.7} \mathrm{Ca}_{0.3} \mathrm{MnO}_{3}{ }^{\prime \prime}$. In: 3 (2013), p. 1704. DOI: $10.1038 /$ s rep 01704.

[22] H. S. Lee and H. H. Park. "The observation of valence band change on resistive switching of epitaxial $\mathrm{Pr}_{0.7} \mathrm{Ca}_{0.3} \mathrm{MnO}_{3}$ film using removable liquid electrode". In: Applied Physics Letters 107 (23) (2015), pp. 1-6. DOI: $10.1063 /$ 1.4937011.

[23] C. Lenser, A. Koehl, I. Slipukhina, H. Du, M. Patt, V. Feyer, C. M. Schneider, M. Lezaic, R. Waser, and R. Dittmann. "Formation and movement of cationic defects during forming and resistive switching in $\mathrm{SrTiO}_{3}$ Thin Film devices". In: Advanced Functional Materials 25 (40) (2015), pp. 6360-6368. DOI: 10.1002 /adfm.201500851.

[24] K. Szot, W. Speier, G. Bihlmayer, and R. Waser. "Switching the electrical resistance of individual dislocations in single-crystalline SrTiO3." In: Nature materials 5 (4) (2006), pp. 312-20. DOI: $10.1038 /$ nmat 1614.

[25] F. Messerschmitt, M. Kubicek, S. Schweiger, and J. L. M. Rupp. “Memristor kinetics and diffusion characteristics for mixed anionic-electronic $\mathrm{SrTiO} 3$ bits: The memristor-based cottrell analysis connecting material to device performance". In: Advanced Functional Materials 24 (47) (2014), pp. 7448 7460. DOI: $10.1002 /$ adfm.201402286.

[26] F. Messerschmitt, M. Kubicek, and J. L. M. Rupp. "How does moisture affect the physical property of memristance for anionic-electronic resistive switching memories?" In: Advanced Functional Materials 25 (32) (2015), pp. 51175125. DOI: $10.1002 / \mathrm{adfm} .201501517$.

[27] X. Lian, X. Cartoixa, E. Miranda, L. Perniola, R. Rurali, S. Long, M. Liu, and Jordi Sune. "Multi-scale quantum point contact model for filamentary conduction in resistive random access memories devices". In: Journal of Applied Physics 115 (24) (2014). DOI: $10.1063 / 1.4885419$. 
[28] I. Valov, R. Waser, J. R. Jameson, and M. N. Kozicki. “Electrochemical metallization memories-fundamentals, applications, prospects." In: Nanotechnology 22 (25) (2011), p. 254003. DOI: $10.1088 / 0957-4484 / 22 / 28 / 289502$.

[29] R. Buzio, A. Gerbi, A. Gadaleta, L. Anghinolfi, F. Bisio, E. Bellingeri, A. S. Siri, and D. Marre. "Modulation of resistance switching in $\mathrm{Au} / \mathrm{Nb}: \mathrm{SrTiO}_{3}$ Schottky junctions by ambient oxygen". In: Applied Physics Letters 101 (24) (2012). DOI: $10.1063 / 1.4771603$.

[30] S. Tsui, A. Baikalov, J. Cmaidalka, Y. Y. Sun, Y. Q. Wang, Y. Y. Xue, C. W. Chu, L. Chen, and A. J. Jacobson. "Field-induced resistive switching in metaloxide interfaces". In: Applied Physics Letters 85 (2) (2004), pp. 317-319. DOI: $10.1063 / 1.1768305$.

[31] T. Oka and N. Nagaosa. "Interfaces of correlated electron systems: Proposed mechanism for colossal electroresistance". In: Physical Review Letters 95 (26) (2005), pp. 1-4. DOI: 10.1103/PhysRevLett.95.266403.

[32] E. Janod, J. Tranchant, B. Corraze, M. Querre, P. Stoliar, M. Rozenberg, T. Cren, D. Roditchev, V. T. Phuoc, M. P. Besland, and L. Cario. "Resistive Switching in Mott Insulators and Correlated Systems". In: Advanced Functional Materials 25 (40) (2015), pp. 6287-6305. DOI: 10 . 1002 / adfm . 201500823.

[33] D. S. Jeong, R. Thomas, R. S. Katiyar, J. F. Scott, H. Kohlstedt, A. Petraru, and C. S. Hwang. "Emerging memories: resistive switching mechanisms and current status". In: Reports on Progress in Physics 75 (2012), p. 076502. DOI: $10.1088 / 0034-4885 / 75 / 7 / 076502$.

[34] D. S. Jeong, H. Schroeder, and R. Waser. "Coexistence of Bipolar and Unipolar Resistive Switching Behaviors in a $\mathrm{PtTiO}_{2} \mathrm{Pt}$ Stack". In: Electrochemical and Solid-State Letters 10 (8) (2007), G51-G53. DOI: $10.1149 / 1.2742989$.

[35] L. Goux, J. G. Lisoni, M. Jurczak, D. J. Wouters, L. Courtade, and Ch. Muller. "Coexistence of the bipolar and unipolar resistive-switching modes in $\mathrm{NiO}$ cells made by thermal oxidation of Ni layers". In: Journal of Applied Physics 107 (2) (2010). DOI: $10.1063 / 1.3275426$.

[36] S. Xianwen, L. Guoqiang, Z. Xin'an, D. Linghong, and Z. Weifeng. "Coexistence of the bipolar and unipolar resistive switching behaviours in $\mathrm{Au} / \mathrm{SrTiO}_{3} / \mathrm{Pt}$ cells". In: 44 (12) (2011), p. 125404. DOI: $10.1088 / 0022-$ $3727 / 44 / 12 / 125404$.

[37] T. Yanagida, K. Nagashima, K. Oka, Ma. Kanai, A. Klamchuen, B. H. Park, and T. Kawai. "Scaling effect on unipolar and bipolar resistive switching of metal oxides." In: Scientific reports 3 (2013), p. 1657. DOI: 10 . 1038 / srep 01657.

[38] F. Gomez-Marlasca, N. Ghenzi, M. J. Rozenberg, and P. Levy. “Understanding electroforming in bipolar resistive switching oxides". In: Applied Physics Letters 98 (4) (2011), p. 042901. DOI: $10.1063 / 1.3537957$.

[39] J. Joshua Yang, F. Miao, M. D. Pickett, D. A. A. Ohlberg, D. R. Stewart, C. N. Lau, and R. S. Williams. "The mechanism of electroforming of metal oxide memristive switches." In: Nanotechnology 20 (21) (2009), p. 215201. DOI: $10.1088 / 0957-4484 / 21 / 33 / 339803$.

[40] E. Lortscher, J. W. Ciszek, J. Tour, and H. Riel. "Reversible and controllable switching of a single-molecule junction". In: Small 2 (8-9) (2006), pp. 973977. DOI: $10.1002 / \mathrm{smll} .200600101$.

[41] E. Y. Tsymbal and H. Kohlstedt. "Tunneling across a ferroelectric". In: Science 313 (5784) (2006), pp. 181-3. DOI: $10.1126 /$ science. 1126230. 
[42] S. Raoux, F. Xiong, M. Wuttig, and E. Pop. "Phase change materials and phase change memory". In: MRS Bulletin 39 (2014), pp. 703-710. DOI: 10 . $1557 / \mathrm{mrs} .2014 .139$.

[43] D. Ielmini, R. Bruchhaus, and R. Waser. "Thermochemical resistive switching: materials, mechanisms, and scaling projections". In: Phase Transitions 84 (7) (2011), pp. 570-602. DOI: $10.1080 / 01411594.2011 .561478$.

[44] J. G. Simmons and R. R. Verderber. "New Conduction and Reversible Memory Phenomena in Thin Insulating Films". In: Proceedings of the Royal Society of London. Series A. Mathematical and Physical Sciences 301 (1464) (1967), 77 LP $-102$.

[45] A. Sawa, T. Fujii, M. Kawasaki, and Y. Tokura. "Interface resistance switching at a few nanometer thick perovskite manganite active layers". In: Applied Physics Letters 88 (23) (2006), pp. 2004-2007. DOI: $10.1063 / 1.2211147$.

[46] A Asamitsu, Y Tomioka, H Kuwahara, and Y Tokura. "Current switching of resistive states in magnetoresistive manganites". In: Nature 388 (6637) (1997), pp. 50 -2. DOI: http: / /dx.doi.org/10.1038/40363.

[47] P. Stoliar, L. Cario, E. Janod, B. Corraze, C. Guillot-Deudon, S. SalmonBourmand, V. Guiot, J. Tranchant, and M. Rozenberg. "Universal electricfield-driven resistive transition in narrow-gap Mott insulators." In: Advanced materials 25 (23) (2013), pp. 3222-6. DOI: 10 . 1002 / adma . 201301113.

[48] Ch. Jooss, L. Wu, T. Beetz, R. F. Klie, M. Beleggia, M. A. Schofield, S. Schramm, J. Hoffmann, and Y. Zhu. "Polaron melting and ordering as key mechanisms for colossal resistance effects in manganites." In: Proceedings of the National Academy of Sciences of the United States of America 104 (34) (2007), pp. 13597-602. DOI: 10.1073 /pnas.0702748104.

[49] A. Sawa. "Resistive switching in transition metal oxides". In: Materials Today 11 (6) (2008), pp. 28-36.

[50] C. Moreno, C. Munuera, S. Valencia, F. Kronast, X. Obradors, and C. Ocal. "Reversible resistive switching and multilevel recording in $\mathrm{La}_{0.7} \mathrm{Sr}_{0.3} \mathrm{MnO}_{3}$ thin films for low cost nonvolatile memories". In: Nano Letters 10 (10) (2010), pp. 3828-3835. DOI: 10.1021/n11008162.

[51] Cesar Moreno. "New features in solution derived $\mathrm{La}_{0.7} \mathrm{Sr}_{0.3} \mathrm{MnO}_{3}$ thin films : spontaneous outcropping and nanoscale reversible resistive switching". PhD thesis. Universitat Autonoma de Barcelona, 2010.

[52] N. F. Mott. "Metal-insulator transition". In: Reviews of Modern Physics 40 (4) (1968), pp. 677-683. DOI: $10.1103 /$ RevModPhys . 40.677.

[53] N. F. Mott. Mott90book-chap8.pdf. 2nd. London: Taylor Francis, 1990.

[54] Florian Gebhard. The Mott Metal-Insulator Transition: Models and Methods. London / Berlin: Springer, 1997, p. 317.

[55] M. Imada, A. Fujimori, and Y. Tokura. "Metal-insulator transitions". In: Reviews of Modern Physics 70 (4) (1998), pp. 1039-1263. DOI: $10.1103 /$ RevModPhys.70.1039.

[56] Z. Yang, C. Ko, and S. Ramanathan. "Oxide electronics utilizing ultrafast Metal-Insulator Transitions". In: Annual Review of Materials Research 41 (1) (2011), pp. 337-367. DOI: 10.1146/annurev-matsci-062910-100347.

[57] You Zhou and Shriram Ramanathan. "Correlated electron materials and field effect transistors for logic: a review". In: Critical reviews in solid state and materials sciences 38 (4) (2013), pp. 286-317. DOI: $10.1080 / 10408436$. 2012.719131. 
[58] P. W. Anderson. "Absence of diffusion in certain random lattices". In: Physical Review 109 (5) (1958), pp. 1492-1505. DOI: 10.1103 / PhysRev . 109. 1492.

[59] G. A. Thomas, Y. Ootuka, S. Katsumoto, S. Kobayashi, and W. Sasaki. "Evidence for localization effects in compensated semiconductors". In: Physical Review B 25 (6 1982), pp. 4288-4290. DOI: 10.1103 / PhysRevB. 25.4288.

[60] M. A. Paalanen, T. F. Rosenbaum, G. A. Thomas, and R. N. Bhatt. "Stress tuning of the metal-insulator transition at millikelvin temperatures". In: Physical Review Letters 48 (18 1982), pp. 1284-1287. DOI: 10 . 1103 / PhysRevLett. 48.1284.

[61] S. Waffenschmidt, C. Pfleiderer, and H. v. Lohneysen. "Critical behaviour of the conductivity of Si:P at the metal-insulator transition under uniaxial stress". In: Physical Review Letters 83 (15 1999), pp. 3005-3008. DOI: 10 . 1103 /PhysRevLett.83.3005.

[62] R. E. Peierls. Quantum theory of solids. Oxford: Clarendon Press, 1956, p. 229.

[63] O. Groning, O. M. Kuttel, P. Groning, and L. Schlapbach. "Field emission from DLC films". In: Applied Surface Science 111 (1997), pp. 135-139. DOI: 10.1016/S0169-4332(96)00713-1.

[64] J. Chaussy, P. Haen, J.C. Lasjaunias, P. Monceau, G. Waysand, A. Waintal, A. Meerschaut, P. Molinie, and J. Rouxel. "Phase transitions in $\mathrm{NbSe}_{3}$ ". In: Solid State Communications 20 (8) (1976), pp. 759-763. DOI: $10.1016 / 0038$ 1098 ( 76 ) 90289-1.

[65] W. Fogle and J. H. Perlstein. "Semiconductor-to-metal transition in the blue potassium molybdenum bronze, $\mathrm{K}_{0.30} \mathrm{MoO}_{3}$; example of a possible excitonic insulator". In: Physical Review B 6 (4 1972), pp. 1402-1412. DOI: $10.1103 /$ PhysRevB.6.1402.

[66] N. F. Mott. "The Basis of the electron theory of metals, with special reference to the transition metals". In: Proceedings of the Physical Society. Section A 62 (7) (1949), pp. 416-422. DOI: $10.1088 / 0370-1298 / 62 / 7 / 303$.

[67] J. Hubbard. "Electron correlations in narrow energy bands. II. The degenerate band case". In: Proceedings of the Royal Society of London A: Mathematical, Physical and Engineering Sciences 277 (1369) (1964), pp. 237-259.

[68] L. Medarde. "Structural, magnetic and electronic properties of perovskites $\left(\mathrm{R}=\right.$ rare earth): structural , magnetic and electronic properties of $\mathrm{RNiO}_{3}$ perovskites". In: Journal of Physics: Condensed Matter 9 (1997), pp. 1679-1707. DOI: $10.1088 / 0953-8984 / 9 / 8 / 003$.

[69] X. Obradors, L.M. Paulius, M. B. Maple, J.B. Torrance, A.I. Nazzal, J. Fontcuberta, and X. Granados. "Pressure dependence of the metal-insulator transition in the charge-transfer oxides $\mathrm{RNiO}_{3}\left(\mathrm{R}=\mathrm{Pr}, \mathrm{Nd}, \mathrm{Nd}_{0.7} \mathrm{La}_{0.3}\right)^{\prime \prime}$. In: Physical Review B 47 (18) (1993), pp. 353-356. DOI: https: / / doi.org/10.1103/ PhysRevB.47.12353.

[70] K. Gupta, B. Mandal, and P. Mahadevan. "Strain-induced metal-insulator transition in ultrathin films of $\mathrm{SrRuO}_{3}$ ". In: Physical Review B 90 (12) (2014), p. 125109. DOI: 10.1103/PhysRevB.90.125109.

[71] S. Catalano, M. Gibert, V. Bisogni, O. E. Peil, F. He, R. Sutarto, M. Viret, P. Zubko, R. Scherwitzl, A. Georges, G. A. Sawatzky, T. Schmitt, and J. M. Triscone. "Electronic transitions in strained $\mathrm{SmNiO}_{3}$ thin films". In: APL Materials 2 (11) (2014). DOI: $10.1063 / 1.4902138$.

[72] R. S. Dhaka, Tanmoy Das, N. C. Plumb, Z. Ristic, W. Kong, C. E. Matt, N. Xu, Kapildeb Dolui, E. Razzoli, M. Medarde, L. Patthey, M. Shi, M. Radovic, and 
Joel Mesot. "Tuning the metal-insulator transition in $\mathrm{NdNiO}_{3}$ heterostructures via Fermi surface instability and spin fluctuations". In: Physical Review B 92 (3) (2015), p. 035127. DOI: $10.1103 /$ PhysRevB.92.035127.

[73] A. Urushibara, T. Arima, A. Asamitsu, G. Kido, and Y. Tokura. "Insulatormetal transition and giant magnetoresistance in $\mathrm{La}_{1-x} \mathrm{Sr}_{x} \mathrm{MnO}_{3}$ ". In: Physical Review B 51 (20) (1995), pp. 14103-14109. DOI: 10.1103 / Phys RevB . 51.14103.

[74] R. Liang, D. A. Bonn, and W. N. Hardy. "Evaluation of $\mathrm{CuO}_{2}$ plane hole doping in $\mathrm{YBa}_{2} \mathrm{Cu}_{3} \mathrm{O}_{6+x}$ single crystals". In: Physical Review B 73 (18) (2006), pp. 1-4. DOI: 10.1103 /PhysRevB.73.180505.

[75] M. A. Hossain, J. D. F. Mottershead, D. Fournier, A. Bostwick, J. L. McChesney, E. Rotenberg, R. Liang, W. N. Hardy, G. A. Sawatzky, I. S. Elfimov, D. A. Bonn, and A. Damascelli. "In situ doping control of the surface of hightemperature superconductors". In: Nature Physics 4 (7) (2008), pp. 527-531. DOI: $10.1038 /$ nphys 998.

[76] A. Chikamatsu, H. Wadati, H. Kumigashira, M. Oshima, A. Fujimori, M. Lippmaa, K. Ono, M. Kawasaki, and H. Koinuma. "Gradual Disappearance of the Fermi Surface near the Metal-Insulator Transition in $\mathrm{La}_{1-x} \mathrm{Sr}_{x} \mathrm{MnO}_{3}$ ". In: Physical Review B 76 (2007), p. 201103. DOI: 10.1103 / PhysRevB . 76 . 201103.

[77] J. Torrance, P. Lacorre, A. Nazzal, E. Ansaldo, and Ch. Niedermayer. "Systematic study of insulator-metal transitions in perovskites $\mathrm{RNiO}_{3}$ $(\mathrm{R}=\mathrm{Pr}, \mathrm{Nd}, \mathrm{Sm}, \mathrm{Eu})$ due to closing of charge-transfer gap". In: Physical Review B 45 (14) (1992), pp. 8209-8212. DOI: 10.1103 / PhysRevB. 45.8209.

[78] S. Catalano, M. Gibert, V. Bisogni, F. He, R. Sutarto, M. Viret, P. Zubko, R. Scherwitzl, G. A Sawatzky, T. Schmitt, and J.M. Triscone. "Tailoring the electronic transitions of NdNiO3 films through ( 111 ) pc oriented interfaces". In: APL Materials 3 (May) (2015), p. 062506. DOI: $10.1063 / 1.4919803$.

[79] I. H. Inoue and M. J. Rozenberg. "Taming the Mott Transition for a Novel Mott Transistor". In: Advanced Functional Materials 18 (16) (2008), pp. 2289 2292. DOI: $10.1002 / \mathrm{adfm} .200800558$.

[80] V. Dubost, T. Cren, C. Vaju, L. Cario, E. Janod, and D. Roditchev. "Resistive Switching at the Nanoscale in the Mott Insulator Compound $\mathrm{GaTa}_{4} \mathrm{Se}_{8}$ ". In: Nano Lett. 13 (2013), pp. 3648 -3653. DOI: dx . doi . org/10 . 1021 / n1401510p.

[81] V. Guiot, L. Cario, E. Janod, B. Corraze, V. T. Phuoc, M. Rozenberg, P. Stoliar, T. Cren, and D. Roditchev. "Avalanche breakdown in $\mathrm{GaTa}_{4} \mathrm{Se}_{8-x} \mathrm{Te}_{x}$ narrow-gap Mott insulators." In: Nature communications 4 (2013), p. 1722. DOI: $10.1038 /$ ncomms 2735 .

[82] J. Wang, J. B. Neaton, H. Zheng, V. Nagarajan, S. B. Ogale, B. Liu, D. Viehland, V. Vaithyanathan, D. G. Schlom, U. V. Waghmare, N. A. Spaldin, K. M. Rabe, M. Wuttig, and R. Ramesh. "Epitaxial $\mathrm{BiFeO}_{3}$ multiferroic thin film heterostructures." In: Science 299 (5613) (2003), pp. 1719-1722. DOI: 10.1126/science.1080615.

[83] D. I. Khomskii and G. A. Sawatzky. "Interplay between spin, charge and orbital degrees of freedom in magnetic oxides". In: Solid State Communications 102 (2-3) (1997), pp. 87-99. DOI: 10.1016/S $0038-1098$ (96) $00717-X$.

[84] E. Dagotto, T. Hotta, and A. Moreo. "Colossal magnetoresistant materials: the key role of phase separation". In: 344 (1-3) (2001), pp. 1-153. DOI: 10 . 1016/S0370-1573(00)00121-6. 
[85] J.G. Bednorz and K.A. Muller. "Possible high $\mathrm{T}_{c}$ superconductivity in the Ba-La-Cu-O system". In: Zeitschrift fuer Physik B: Condensed Matter 64 (1986), pp. 189-193. DOI: $10.1007 /$ BF 01303701.

[86] G.H. Jonker and J.H. Van Santen. "Ferromagnetic compounds of manganese with perovskite structure". In: Physica XVI 16 (3) (1950), pp. 337-349. DOI: 10.1016/0031-8914(50)90033-4.

[87] R. Von Helmolt, J. Wecker, B. Holzapfel, L. Schultz, and K. Samwer. "Giant negative magnetoresistance in perovskite like $\mathrm{La}_{2 / 3} \mathrm{Ba}_{1 / 3} \mathrm{MnO}_{3}$ ferromagnetic films". In: Physical Review Letters 71 (14) (1993), pp. 2331-2333. DOI: https://dx.doi.org/10.1103/PhysRevLett.71.2331.

[88] S. Jin, T. H. Tiefel, M. McCormack, R. A. Fastnacht, R. Ramesh, and L. H. Chen. "Thousandfold change in resistivity in magnetoresistive La-Ca-Mn-O Films". In: Science 264 (5157) (1994), pp. 413-415. DOI: 10.1126 / science. 264.5157 .413$.

[89] A.P. Ramirez. "Colossal magnetoresistance". In: Journal of Physics: Condensed Matter 9 (39) (1997), pp. 8171-8199. DOI: https : / / doi . org/10 . 1088 / 0953-8984/9/39/005.

[90] M. B Salamon and M. Jaime. "The physics of manganites : Structure and transport". In: Review of Modern Physics 73 (July) (2001), pp. 583-628.

[91] Y Tokura. "Critical features of colossal magnetoresistive manganites". In: Reports on Progress in Physics 69 (3) (2006), pp. 797-851. DOI: 10.1088 / 0034-4885/69/3/R06.

[92] H. A. Jahn and E. Teller. "Stability of polyatomic molecules in degenerate electronic states. I. Orbital Degeneracy". In: Proceedings of the Royal Society A: Mathematical and Physical Sciences 16t1 (906) (1937), pp. 220-235. DOI: $10.1098 /$ rspa.1937.0142.

[93] Clarence Zener. "Interaction between the d-Shells in the Transition Metals. II. Ferromagnetic compounds of manganese with perovskite structure". In: Physical Review B 82 (3) (1951), pp. 403-405. DOI: 10.1103 / PhysRev . 82. 403.

[94] H. Y. Hwang, S. W. Cheong, P. G. Radaelli, M. Marezio, and B. Batlogg. "Lattice effects on the magnetoresistance in doped $\mathrm{LaMnO}_{3}{ }^{\prime \prime}$. In: Physical Review Letters 75 (5) (1995), pp. 914-917. DOI: 10.1103 / PhysRevLett. 75.914.

[95] A.S. Alexandrov and A.M. Bratkovsky. "Theory of colossal magnetoresistance in doped manganites". In: Journal of Physics: Condensed Matter 11 (8) (1999).

[96] A.S. Alexandrov and A.M. Bratkovsky. "Carrier density collapse and colossal magnetoresistance in doped manganites". In: Physical Review Letters 82 (1) (1999). DOI: 10.1103 / Phy sRevLett. 82.141.

[97] F. Conchon, A. Boulle, R. Guinebretiere, C. Girardot, S. Pignard, J. Kreisel, F. Weiss, E. Dooryhee, and J.-L. Hodeau. "Effect of tensile and compressive strains on the transport properties of $\mathrm{SmNiO}_{3}$ layers epitaxially grown on (001) $\mathrm{SrTiO}_{3}$ and $\mathrm{LaAlO}_{3}$ substrates". In: Applied Physics Letters 91 (19) (2007). DOI: http://dx.doi.org/10.1063/1.2800306.

[98] G. Catalan, R. M. Bowman, and J. M. Gregg. "Transport properties of $\mathrm{NdNiO}_{3}$ thin films made by pulsed-laser deposition". In: Journal of Applied Physics 87 (1) (2000), p. 606. DOI: $10.1063 / 1.371912$.

[99] K. M. Satyalakshmi, R. M. Mallya, K. V. Ramanathan, X. D. Wu, B. Brainard, D. C. Gautier, N. Y. Vasanthacharya, and M. S. Hegde. "Epitaxial metallic $\mathrm{LaNiO}_{3}$ thin films grown by pulsed laser deposition". In: Applied Physics Letters 62 (11) (1993), pp. 1233-1235. DOI: $10.1063 / 1.109612$. 
[100] R. Scherwitzl, S. Gariglio, M. Gabay, P. Zubko, M. Gibert, and J.-M. Triscone. "Metal-Insulator Transition in Ultrathin $\mathrm{LaNiO}_{3}$ films". In: Physical Review Letters 106 (24) (2011), p. 246403. DOI: 10.1103 / PhysRevLett. 106 . 246403.

[101] H. Miyazaki, T. Goto, Y. Miwa, T. Ohno, H. Suzuki, T. Ota, and M. Takahashi. "Preparation and evaluation of $\mathrm{LaNiO}_{3}$ thin film electrode with chemical solution deposition". In: Journal of the European Ceramic Society 24 (6) (2004), pp. 1005-1008. DOI: 10.1016/S0955-2219(03)00382-0.

[102] A. Queralto, M. de la Mata, J. Arbiol, X. Obradors, and T. Puig. “Disentangling epitaxial growth mechanisms of solution derived functional oxide thin films". In: Advanced Materials Interfaces (2016). DOI: 10 . 1002 / admi . 201600392.

[103] G. Catalan. "Progress in perovskite nickelate research". In: Phase Transitions 81 (7-8) (2008), pp. 729-749. DOI: $10.1080 / 01411590801992463$.

[104] J.L. Garcia-Munoz, J. Rodriguez-Carvajal, and P. Lacorre. "Neutrondiffraction study of the magnetic ordering in the insulating regime of the perovskites $\mathrm{RNiO}_{3}(\mathrm{R}=\mathrm{Pr}$ and Nd)". In: Physical Review B 50 (2) (1994).

[105] M. Medarde, C. Dallera, M. Grioni, B. Delley, F. Vernay, J. Mesot, M. Sikora, J. A. Alonso, and M. J. Martinez-Lope. "Charge disproportionation in $\mathrm{RNiO}_{3}$ perovskites". In: Physical Review B 80 (24) (2009), p. 245105. DOI: $10.1103 /$ PhysRevB.80.245105.

[106] U. Staub, G. I. Meijer, F. Fauth, R. Allenspach, J. G. Bednorz, J. Karpinski, S. M. Kazakov, L. Paolasini, and F. D'Acapito. "Direct observation of charge order in an epitaxial $\mathrm{NdNiO}_{3}$ Film". In: Physical Review Letters 88 (12) (2002), p. 126402. DOI: $10.1103 /$ PhysRevLett.88.126402.

[107] V. Scagnoli, U. Staub, A. M. Mulders, M. Janousch, G. I. Meijer, G. Hammerl, J. M. Tonnerre, and N. Stojic. "Role of magnetic and orbital ordering at the metal-insulator transition in $\mathrm{NdNiO}_{3}$ ". In: Physical Review B 73 (10) (2006), pp. 1-4. DOI: 10.1103 /PhysRevB.73.100409.

[108] I. Vobornik, L. Perfetti, M. Zacchigna, M. Grioni, G. Margaritondo, J. Mesot, M. Medarde, and P. Lacorre. "Electronic-structure evolution through the metal-insulator transition in $\mathrm{RNiO}_{3}$ ". In: Physical Review B 60 (12) (1999), R8426. DOI: 10.1103 /PhysRevB.60.R8426.

[109] J.-S. Tsai. "Toward a superconducting quantum computer". In: Proceedings of the Japan Academy, Series B 86 (4) (2010), pp. 275-292. DOI: 10.2183 / pjab.86.275.

[110] Y. Zhang, Y. Tavrin, M. Muck, A.I. Braginski, C. Heiden, S. Hampson, C. Pantev, and T Elbert. "Magnetoencephalography using high temperature rf SQUIDs". In: Brain Topography 5 (4) (1993), pp. 379-382.

[111] S. Anders, M. G. Blamire, F.I. Buchholz, D.-G. Crete, R. Cristiano, P. Febvre, L. Fritzsch, a. Herr, E. Il'ichev, J. Kohlmann, J. Kunert, H.-G. Meyer, J. Niemeyer, T. Ortlepp, H. Rogalla, T. Schurig, M. Siegel, R. Stolz, E. Tarte, H.J.M. ter Brake, H. Toepfer, J.-C. Villegier, Zagoskin A. M., and A. B. Zorin. "European roadmap on superconductive electronics - status and perspectives". In: Physica C: Superconductivity 470 (23-24) (2010), pp. 2079-2126. DOI: $10.1016 / j \cdot$ physc.2010.07.005.

[112] G. W. Mitschang. "Space applications and implications of high temperature superconductivity". In: IEEE Transactions on Applied Superconductivity 5 (2) (1995), pp. 69-73. DOI: $10.1109 / 77.402510$. 
[113] D. Larbalestier, A. Gurevich, D. M. Feldmann, and A. Polyanskii. "High$\mathrm{T}_{c}$ superconducting materials for electric power applications." In: Nature 414 (6861) (2001), pp. 368-377. DOI: $10.1038 / 35104654$.

[114] X. Obradors and T. Puig. "Coated conductors for power applications: materials challenges". In: Superconductor Science and Technology 27 (2014), p. 044003. DOI: $10.1088 / 0953-2048 / 27 / 4 / 044003$.

[115] E. Fradkin and S. A. Kivelson. "High-temperature superconductivity: Ineluctable complexity". In: Nature Physics 8 (12) (2012), pp. 864-866. DOI: $10.1038 /$ nphys2498.

[116] N. Barisic, M. K Chan, Y. Li, G. Yu, X. Z., M. Dressel, A. Smontara, and $\mathrm{M}$. Greven. "Universal sheet resistance and revised phase diagram of the cuprate high-temperature superconductors." In: Proceedings of the National Academy of Sciences of the United States of America 110 (30) (2013), pp. 1223540. DOI: $10.1073 /$ pnas. 1301989110.

[117] M. Tinkham. Introduction to superconductivity. Second Edition. Dover Publications, 2004.

[118] J R Waldram. Superconductivity of metals and cuprates. Taylor \& Francis, 1996.

[119] E. Dagotto. "Complexity in strongly correlated electronic systems." In: Science 309 (5732) (July 2005), pp. 257-62. DOI: 10.1126 / science. 1107559.

[120] R. Guzman, J. Gazquez, V. Rouco, A. Palau, C. Magen, M. Varela, J. Arbiol, $\mathrm{X}$. Obradors, and T. Puig. "Strain-driven broken twin boundary coherence in $\mathrm{YBa}_{2} \mathrm{Cu}_{3} \mathrm{O}_{7-\delta}$ nanocompositethinfilms". In: Applied Physics Letters 102 (8) (2013). DOI: $10.1063 / 1.4793749$.

[121] J. D. Jorgensen, B. W. Veal, a. P. Paulikas, L.J. Nowicki, G. W. Crabtree, H. Claus, and W. K. Kwok. "Structural properties of oxygen-deficient $\mathrm{YBa}_{2} \mathrm{Cu}_{3} \mathrm{O}_{7-\delta}$ ". In: Physical Review B 41 (4) (1990), p. 1863. DOI: $10.1103 /$ PhysRevB.41.1863.

[122] L. Malavasi, C. A. J. Fisher, and M. S. Islam. "Oxide-ion and proton conducting electrolyte materials for clean energy applications: structural and mechanistic features". In: Chemical Society Reviews 39 (11) (2010), pp. 43704387. DOI: $10.1039 /$ b915141a.

[123] C. H. Cheng, H. H. Hsu, W. B. Chen, Albert Chin, and F. S. Yeh. “Characteristics of cerium oxide for metal-insulator-metal capacitors". In: Electrochemical and Solid-State Letters 13 (1) (2010), H16. DOI: $10.1149 / 1.3258042$.

[124] S. Schweiger, M. Kubicek, F. Messerschmitt, C. Murer, and J. L. M. Rupp. "A microdot multilayer oxide device: Let us tune the strain-ionic transport interaction". In: ACS Nano 8 (5) (2014), pp. 5032-5048. DOI: $10.1021 /$ nn501128y.

[125] X. Obradors, T. Puig, A. Pomar, F. Sandiumenge, N. Mestres, M. Coll, A. Cavallaro, N. Roma, J. Gazquez, J. C. Gonzalez, O. Castano, J. Gutierrez, A. Palau, K. Zalamova, S. Morlens, A. Hassini, M. Gibert, S. Ricart, J. M. Moreto, S. Pinol, D. Isfort, and J. Bock. "Progress towards all-chemical superconducting $\mathrm{YBa}_{2} \mathrm{Cu}_{3} \mathrm{O}_{7}$-coated conductors". In: Superconductor Science and Technology 19 (3) (2006), S13.

[126] B. C. Steele and A. Heinzel. "Materials for fuel-cell technologies." In: Nature 414 (11) (2001), pp. 345-352. DOI: $10.1038 / 35104620$.

[127] M. Mogensen, N. M. Sammes, and G. A. Tompsett. "Physical, chemical and electrochemical properties of pure and doped ceria". In: Solid State Ionics 129 (1) (2000), pp. 63-94. DOI: $10.1016 /$ S0167-2738 (99) 00318-5. 
[128] S. P. S. Badwal, D. Fini, F. T. Ciacchi, C. Munnings, J. A. Kimpton, and J. Drennan. "Structural and microstructural stability of ceria - gadolinia electrolyte exposed to reducing environments of high temperature fuel cells". In: Journal of Materials Chemistry A 1 (36) (2013), p. 10768. DOI: 10.1039 / c3ta11752a.

[129] D. A. Andersson, S. I. Simak, N. V. Skorodumova, I. A Abrikosov, and B. Johansson. "Optimization of ionic conductivity in doped ceria." In: Proceedings of the National Academy of Sciences of the United States of America 103 (10) (2006), pp. 3518-21. DOI: $10.1073 /$ pnas. 0509537103.

[130] A Ohtomo and H Y Hwang. "A high-mobility electron gas at the LAO/STO heterointerface." In: Nature 427 (6973) (2004), pp. 423-426. DOI: $10.1038 /$ nature 02308.

[131] J. Garcia-Barriocanal, A. Rivera-Calzada, M. Varela, Z. Sefrioui, E. Iborra, C. Leon, S. J. Pennycook, and J. Santamaria. "Colossal ionic conductivity at interfaces of epitaxial $\mathrm{ZrO}_{2}: \mathrm{Y}_{2} \mathrm{O}_{3} / \mathrm{SrTiO}_{3}$ heterostructures". In: Science 321 (5889) (2008), pp. 676-680. DOI: $10.1126 /$ science. 1156393.

[132] J. M. Phillips. "Substrate selection for high-temperature superconducting thin films". In: Journal of Applied Physics 79 (4) (1996), p. 1829. DOI: 10 . $1063 / 1.362675$.

[133] D. Pesquera, G. Herranz, A. Barla, E. Pellegrin, F. Bondino, E. Magnano, F. Sanchez, and J. Fontcuberta. "Surface symmetry-breaking and strain effects on orbital occupancy in transition metal perovskite epitaxial films". In: $\mathrm{Na}$ ture Communications 3 (2012). DOI: 10.1038 / ncomms 2189.

[134] B. Dam, J. M. Huijbregtse, and J. H. Rector. "Strong pinning linear defects formed at the coherent growth transition of pulsed-laser-deposited $\mathrm{YBa}_{2} \mathrm{Cu}_{3} \mathrm{O}_{7-\delta}$ films". In: Physycal Review B 65 (6 2002), p. 064528. DOI: $10.1103 /$ PhysRevB.65.064528.

[135] S. Gariglio, N. Reyren, A. D. Caviglia, and J.-M. Triscone. "Superconductivity at the $\mathrm{LaAlO}_{3} / \mathrm{SrTiO}_{3}$ interface". In: Journal of Physics: Condensed Matter 21 (16) (2009), p. 164213. DOI: $10.1088 / 0953-8984 / 21 / 16 / 164213$.

[136] Y. S. Kim, D. J. Kim, T. H. Kim, T. W. Noh, J. S. Choi, B. H. Park, and J.G. Yoon. "Observation of room-temperature ferroelectricity in tetragonal strontium titanate thin films on SrTiO3 (001) substrates". In: Applied Physics Letters 91 (4) (2007). DOI: http://dx.doi.org/10.1063/1.2764437.

[137] R. A. McKee, F. J. Walker, and M. F. Chisholm. "Physical structure and inversion charge at a semiconductor interface with a crystalline oxide". In: Science 293 (5529) (2001), pp. 468-471. DOI: 10.1126 / science. 293. 5529.468.

[138] R. A. McKee, F. J. Walker, and M. F. Chisholm. "Crystalline oxides on silicon: The tirst five monolayers". In: Physical Review Letters 81 (14 1998), pp. 30143017. DOI: 10.1103 /PhysRevLett.81.3014.

[139] K. Szot, R. Dittmann, W. Speier, and R. Waser. "Nanoscale resistive switching in SrTiO3 thin films". In: physica status solidi (RRL) - Rapid Research Letters 1 (2) (2007), R86-R88. DOI: 10.1002 /pssr. 200701003.

[140] S. Menzel, M. Waters, A. Marchewka, U. Bottger, R. Dittmann, and R. Waser. "Origin of the ultra-nonlinear switching kinetics in oxide-based resistive switches". In: Advanced Functional Materials 21 (23) (2011), pp. 4487-4492. DOI: 10.1002 /adfm.201101117.

[141] H R Mitchell, R A Chakhmouradian, and M P Woodward. "Crystal chemistry of perovskite-type compounds in the tausonite-loparite series, 
$\left(\mathrm{Sr}_{12 x} \mathrm{Na}_{x} \mathrm{La}_{x}\right) \mathrm{TiO}_{3}{ }^{\prime \prime} . \quad$ In: Physics and Chemistry of Minerals 27 (8) (2000), pp. 583-589. DOI: $10.1007 / \mathrm{s} 002690000103$.

[142] S. Bueble, K. Knorr, E. Brecht, and W. W. Schmahl. "Influence of the ferroelastic twin domain structure on the 100 surface morphology of $\mathrm{LaAlO}_{3}$ HTSC substrates". In: Surface science 400 (1-3) (), pp. 345-355. DOI: http : //dx.doi.org/10.1016/S0039-6028(97)00891-1.

[143] G. Koster, B. L. Kropman, G. J. H. M. Rijnders, D. H. A. Blank, and H. Rogalla. "Quasi-ideal strontium titanate crystal surfaces through formation of strontium hydroxide". In: Applied Physics Letters 73 (20) (1998). DOI: $10.1063 / 1.122630$.

[144] Z. L. Wang. "Steps and facets on annealed $\mathrm{LaAlO}_{3} 100$ and 110 surfaces". In: Surface Science 360 (1) (1996), pp. 180-186. DOI: http: / / dx . doi . org/ 10 . 1016/0039-6028(96)00583-3.

[145] J Zabaleta. "Growth and advanced characterization of solution-derived nanoscale $\mathrm{La}_{0.7} \mathrm{Sr}_{0.3} \mathrm{MnO}_{3}$ heteroepitaxial systems". $\mathrm{PhD}$ thesis. Universitat Autonoma de Barcelona, 2012.

[146] Albert Queralto. "Growth of functional oxide heterostructures from chemical solutions using advanced processing methodologies". PhD thesis. Universitat Autonoma de Barcelona, 2015.

[147] A.-M. Haghiri-Gosnet and J.-P. Renard. "CMR manganites: physics, thin films and devices". In: Journal of Physics D: Applied Physics 36 (8) (2003), R127-R150. DOI: 10.1088/0022-3727/36/8/201.

[148] C. Moreno, P. Abellan, A. Hassini, A. Ruyter, A. Perez del Pino, F. Sandiumenge, M.-J. Casanove, J. Santiso, T. Puig, and X. Obradors. "Spontaneous outcropping of self-sssembled insulating nanodots in solutionderived metallic ferromagnetic $\mathrm{La}_{0.7} \mathrm{Sr}_{0.3} \mathrm{MnO}_{3}$ films". In: Advanced Functional Materials 19 (13) (2009), pp. 2139-2146. DOI: 10 . 1002 / adfm . 200900095.

[149] R W Schwartz. "Chemical solution deposition of perovskite thin films". In: Chemistry of Materials 9 (11) (1997), pp. 2325-2340. DOI: Doi10.1021/ Cm970286f.

[150] R. W. Schwartz, T. Schneller, and R. Waser. "Chemical solution deposition of electronic oxide films". In: Comptes Rendus Chimie 7 (5) (2004), pp. 433461.

[151] "Chemical solution route to self-assembled epitaxial oxide nanostructures." In: Chemical Society Reviews 43 (7) (2014), pp. 2200-25. DOI: 10 . 1039 / c3cs60365b.

[152] X. Obradors, T. Puig, A. Pomar, F. Sandiumenge, N. Mestres, M. Coll, A. Cavallaro, N. Roma, J. Gazquez, J. C. Gonzalez, O. Castano, J. Gutierrez, A. Palau, K. Zalamova, S. Morlens, A. Hassini, M. Gibert, S. Ricart, J. M. Moreto, S. Pinol, D. Isfort, and J. Bock. "Progress towards all-chemical superconducting $\mathrm{YBa}_{2} \mathrm{Cu}_{3} \mathrm{O}_{7}$-coated conductors". In: Superconductor Science and Technology 19 (3) (2006), S13. DOI: https:// doi .org/10 . 1088 / 0953-2048/19/3/003.

[153] A. Queralto, A. Perez del Pino, M. de la Mata, J. Arbiol, M. Tristany, A. Gomez, X. Obradors, and T. Puig. "Growth of ferroelectric $\mathrm{Ba}_{0.8} \mathrm{Sr}_{0.2} \mathrm{TiO}_{3}$ epitaxial films by ultraviolet pulsed laser irradiation of chemical solution derived precursor layers". In: Applied Physics Letters 106 (26) (2015), p. 262903. 
[154] C. Moreno, P. Abellan, F. Sandiumenge, M.-J. Casanove, and X. Obradors. "Nanocomposite lanthanum strontium manganite thin films formed by using a chemical solution deposition". In: Applied Physics Letters 100 (2) (2012). DOI: http://dx.doi.org/10.1063/1.3675461.

[155] J. Zabaleta, M. Jaafar, P. Abellan, C. Monton, O. Iglesias-Freire, F. Sandiumenge, C. A Ramos, R. D. Zysler, T. Puig, A. Asenjo, N. Mestres, and $X$. Obradors. "Nanoscale magnetic structure and properties of solutionderived self-assembled $\mathrm{La}_{0.7} \mathrm{Sr}_{0.3} \mathrm{MnO}_{3}$ islands". In: Journal of Applied Physics 111 (2) (2012). DOI: http://dx.doi.org/10.1063/1.3677985.

[156] Rafael Ortega-Hernandez. "Resistive Memory Devices based on Complex Oxides". PhD thesis. Universitat Autònoma de Barcelona, 2015, p. 153.

[157] L. J. Van der Pauw. "A method of measuring the resistivity and hall coefficient on laellae of arbitrary shape". In: Philips Technical Review 13 (1) (1958), pp. 1-9.

[158] A. Monsen, J. E. Boschker, F. Macia, J. W. Wells, P. Nordblad, A. D. Kent, R. Mathieu, T. Tybell, and E. Wahlstrom. "Thickness dependence of dynamic and static magnetic properties of pulsed laser deposited $\mathrm{La}_{0.7} \mathrm{Sr}_{0.3} \mathrm{MnO}_{3}$ films on $\mathrm{SrTiO}_{3}$ (001)". In: Journal of Magnetism and Magnetic Materials 369 (2014), pp. 197 -204. DOI: $10.1016 / j$.jmmm.2010.12.007.

[159] B. Kim, D. Kwon, J. Hyun, Y. Hikita, B. G. Kim, and H. Y. Hwang. "Finite size effect and phase diagram of ultra-thin $\mathrm{La}_{0.7} \mathrm{Sr}_{0.3} \mathrm{MnO}_{3}$ ". In: Solid State Communications 150 (13-14) (2010), pp. 598-601. DOI: 10 . 1016/ j.ssc . 2009.12 .041$.

[160] L. Qiao and X. Bi. "Direct observation of oxygen vacancy and its effect on the microstructure, electronic and transport properties of sputtered $\mathrm{LaNiO}_{3 \delta}$ films on Si substrates". In: Thin Solid Films 519 (2) (2010), pp. 943-946. DOI: $10.1016 / j . t s f .2010 .08 .129$.

[161] A Ikeda, T. Manabe, and M. Naito. "Epitaxial strain effect in perovskite $\mathrm{RENiO}_{3}$ films (RE = La-Eu) prepared by metal organic decomposition". In: Physica. C. Superconductivity 505 (), pp. 24-31. DOI: http: / / dx.doi .org/ $10.1016 / j \cdot$ physc.2014.07.005.

[162] N. Ihzaz, S. Pignard, J. Kreisel, H. Vincent, J. Marcus, J. Dhahri, and M. Oumezzine. "Synthesis and characterization of $\mathrm{SmNiO}_{3}$ thin films". In: physica status solidi (c) 1 (7) (2004), pp. 1679-1682. DOI: 10.1002 / pssc . 200304422.

[163] M. Zinkevich and F. Aldinger. "Thermodynamic analysis of the ternary La-Ni-O system". In: Journal of Alloys and Compounds 375 (1-2) (2004), pp. 147-161. DOI: http://dx.doi.org/10.1016/j.jallcom.2003. 11.138.

[164] E. Detemple, Q. M. Ramasse, W. Sigle, G. Cristiani, H. U. Habermeier, B. Keimer, and P. A. Van Aken. "Ruddlesden-Popper faults in $\mathrm{LaNiO}_{3} / \mathrm{LaAlO}_{3}$ superlattices". In: Journal of Applied Physics 112 (1) (2012), pp. 1-6. DOI: $10.1063 / 1.4731249$.

[165] M Lorenz, H Hochmuth, D Natusch, H Borner, G Lippold, K Kreher, and W Schmitz. "Large-area double-side pulsed laser deposition of $\mathrm{YBa}_{2} \mathrm{Cu}_{3} \mathrm{O}_{7-x}$ thin films on 3-in sapphire wafers". In: Applied Physics Letters 68 (23) (1996), pp. 3332-3334. DOI: $10.1063 / 1.116048$.

[166] R. K. Singh and D. Kumar. "Pulsed laser deposition and characterization of high-T-c $\mathrm{YBa}_{2} \mathrm{Cu}_{3} \mathrm{O}_{7-x}$ superconducting thin films". In: 22 (4) (1998), pp. 113-185. DOI: 10.1016/S0927-796X(97)00019-3. 
[167] D. M. Feldmann, O. Ugurlu, B. Maiorov, L. Stan, T. G. Holesinger, L. Civale, S. R. Foltyn, and Q. X. Jia. "Influence of growth temperature on critical current and magnetic flux pinning structures in $\mathrm{YBa}_{2} \mathrm{Cu}_{3} \mathrm{O}_{7-x}$ ". In: Applied Physics Letters 91 (16) (2007), p. 162501. DOI: $10.1063 / 1.2799875$.

[168] A. Goyal, S. Kang, K. J. Leonard, P. M. Martin, A. A. Gapud, M. Varela, M. Paranthaman, A. O. Ijaduola, E. D. Specht, J. R. Thompson, D. K. Christen, S. J. Pennycook, and F. A. List. "Irradiation-free, columnar defects comprised of self-assembled nanodots and nanorods resulting in strongly enhanced flux-pinning in $\mathrm{YBa}_{2} \mathrm{Cu}_{3} \mathrm{O}_{7-\delta}$ films". In: Superconductor Science and Technology 18 (11) (2005), pp. 1533-1538. DOI: $10.1088 / 0953-2048 / 18 / 11 / 021$.

[169] B. Dam, J. Huijbregtse, and J. Rector. "Strong pinning linear defects formed at the coherent growth transition of pulsed-laser-deposited $\mathrm{YBa}_{2} \mathrm{Cu}_{3} \mathrm{O}_{7-\delta}$ films". In: Physical Review B 65 (6) (2002), pp. 1-8. DOI: $10.1103 /$ PhysRevB.65.064528.

[170] B. Maiorov, S. a Baily, H. Zhou, O. Ugurlu, J. a Kennison, P. C. Dowden, T. G. Holesinger, S. R. Foltyn, and L. Civale. "Synergetic combination of different types of defect to optimize pinning landscape using $\mathrm{BaZrO}_{3}$-doped $\mathrm{YBa}_{2} \mathrm{Cu}_{3} \mathrm{O}_{7}$." In: Nature Materials 8 (5) (2009), pp. 398-404. DOI: 10.1038 / nmat2 208.

[171] L. Civale, B. Maiorov, A. Serquis, J.O. Willis, J.Y. Coulter, H. Wang, Q. X. Jia, P. N. Arendt, M. Jaime, J. L. MacManus-Driscoll, M. P. Maley, and S. R. Foltyn. "Understanding high critical currents in YBCO thin films and coated conductors". In: 135 (1/2) (2004), pp. 87-98.

[172] S. R. Foltyn, P. N. Arendt, Q. X. Jia, H. Wang, J. L. MacManus-Driscoll, S. Kreiskott, R. F. DePaula, L. Stan, J. R. Groves, and P. C. Dowden. "Strongly coupled critical current density values achieved in coated YBCO conductors with near-single-crystal texture". In: Applied Physics Letters 82 (25) (2003), pp. 4519-4521. DOI: $10.1063 / 1.1584783$.

[173] R. Eason. Pulsed Laser Deposition of Thin Films. 2007.

[174] H. M. Christen and G. Eres. "Recent advances in pulsed-laser deposition of complex oxides." In: Journal of Physics. Condensed Matter 20 (26) (2008), p. 264005. DOI: $10.1088 / 0953-8984 / 20 / 26 / 264005$.

[175] J Schou. "Physical aspects of the pulsed laser deposition technique: The stoichiometric transfer of material from target to film". In: Applied Surface Science 255 (10) (2009), pp. 5191-5198. DOI: 10 . 1016/j . apsusc. 2008 . 10.101.

[176] B. Dam, J. Rector, M. F. Chang, S. Kars, D. G. de Groot, and R. Griessen. "Laser ablation threshold of $\mathrm{YBa}_{2} \mathrm{Cu}_{3} \mathrm{O}_{6+x}$ ". In: Applied Physics Letters 65 (12) (1994), p. 1581. DOI: $10.1063 / 1.112921$.

[177] C. P. Bean. "Magnetization of hard superconductors". In: Physical Review Letters 8 (6) (1962), pp. 250-253. DOI: 10.1103/Phys RevLett. 8 . 250.

[178] Charles P. Bean. "Magnetization of high-field superconductors". In: Reviews of Modern Physics 36 (1) (1964), pp. 31-39. DOI: 10.1103 / RevModPhys . 36.31 .

[179] M. Coll, J. Gazquez, R. Huhne, B. Holzapfel, Y. Morilla, J. Garcia-Lopez, A. Pomar, F. Sandiumenge, T. Puig, and X. Obradors. "All chemical YBa2Cu3O7 superconducting multilayers: Critical role of $\mathrm{CeO}_{2}$ cap layer flatness". In: Journal of Materials Research 24 (4) (2011), pp. 1446-1455. DOI: $10.1557 / \mathrm{jmr} .2009 .0160$. 
[180] Yu. A Boikov, T Claeson, D Erts, F Bridges, and Z Kvitky. " $\mathrm{CeO}_{2}$ compatibility with $\mathrm{YBa}_{2} \mathrm{Cu}_{3} \mathrm{O}_{7-d}$ in superconducting-film multilayers". In: Phys. Rev. B 56 (17) (1997), pp. 11312-11319. DOI: 10.1103 /PhysRevB. 56.11312.

[181] S. V. Kalinin, A. Borisevich, and D. Fong. "Beyond condensed matter physics on the nanoscale: The role of ionic and electrochemical phenomena in the physical functionalities of oxide materials". In: ACS Nano 6 (12) (2012), pp. 10423-10437. DOI: $10.1021 /$ nn304930x.

[182] M. Coll, A. Palau, J. C. Gonzalez-Rosillo, J. Gazquez, X. Obradors, and T. Puig. "Integration of atomic layer deposition $\mathrm{CeO} 2$ thin films with functional complex oxides and 3D patterns". In: Thin Solid Films 553 (2014), pp. 7-12. DOI: $10.1016 / j . t s f .2013 .08 .131$.

[183] S. M. George. "Atomic layer deposition: An overview". In: Chemical Reviews 110 (1) (2010), pp. 111-131. DOI: 10.1021 / cr900056b.

[184] M. D. Groner, F. H. Fabreguette, J. W. Elam, and S. M. George. "LowTemperature Al2O3 Atomic Layer Deposition". In: Chemistry of Materials 16 (4) (2004), pp. 639-645. DOI: 10.1021 / cm0 304546.

[185] M. Coll, J. Gazquez, A. Palau, M. Varela, X. Obradors, and T. Puig. "Low temperature epitaxial oxide ultrathin films and nanostructures by atomic layer deposition". In: Chemistry of Materials 24 (19) (2012), pp. 3732-3737. DOI: $10.1021 / \mathrm{cm} 301864 \mathrm{c}$.

[186] M. Coll, J. M. Montero Moreno, J. Gazquez, K. Nielsch, X. Obradors, and T. Puig. "Low temperature stabilization of nanoscale epitaxial spinel ferrite thin films by atomic layer deposition". In: Advanced Functional Materials 24 (34) (2014), pp. 5368-5374. DOI: 10.1002 /adfm. 201400517.

[187] O. Sneh, R. B. Clark-Phelps, A. R. Londergan, J. Winkler, and T. E. Seidel. "Thin film atomic layer deposition equipment for semiconductor processing". In: Thin Solid Films 402 (1-2) (2002), pp. 248-261. DOI: 10 . $1016 /$ S0040-6090(01)01678-9.

[188] B. J. Choi, D. S. Jeong, S. K. Kim, C. Rohde, S. Choi, J. H. Oh, H. J. Kim, C. S. Hwang, K. Szot, R. Waser, B. Reichenberg, and S. Tiedke. "Resistive switching mechanism of $\mathrm{TiO}_{2}$ thin films grown by atomic-layer deposition". In: Journal of Applied Physics 98 (3) (2005), pp. 1-10. DOI: $10.1063 / 1$. 2001146.

[189] R Zazpe, M. Ungureanu, F. Golmar, P. Stoliar, R. Llopis, F. Casanova, D. F. Pickup, C. Rogero, and L. E Hueso. "Resistive switching dependence on atomic layer deposition parameters in $\mathrm{HfO}_{2}$-based memory devices". In: J. Mater. Chem. C 2 (17) (2014), pp. 3204-3211. DOI: 10.1039 / c3tc31819b.

[190] L.-G. Wang, X. Qian, Y.-Q. Cao, Z.-Y. Cao, G.-Y. Fang, A.-D. Li, and D. $\mathrm{Wu}$. "Excellent resistive switching properties of atomic layer-deposited $\mathrm{Al}_{2} \mathrm{O}_{3} / \mathrm{HfO}_{2} / \mathrm{Al}_{2} \mathrm{O}_{3}$ trilayer structures for non-volatile memory applications". In: Nanoscale Research Letters 10 (1) (2015), p. 135. DOI: $10.1186 /$ s11671-015-0846-y.

[191] L. Pain, S. Tedesco, and C. Constancias. "Direct write lithography: the global solution for R\&D and manufacturing". In: Comptes Rendus Physique 7 (8) (2006), pp.910-923. DOI: $10.1016 /$ j.crhy.2006.10.003.

[192] D M Mattox. Handbook of Physical Vapor Deposition (PVD) Processing. Elsevier Science, 2010.

[193] Binning H. and Rohrer, G. "Scanning Tunnelling Microscopy". In: Helvetica Physica Acta 55 (6) (1982), pp. 726-735. 
[194] G. Binning, C. F. Quate, and C. Gerber. "Atomic Force Microscope”. In: Physical Review Letters 56 (9) (1986), pp. 930-933. DOI: 10.1103 / Phy sRevLett. 56.930 .

[195] Roland Wiesendanger. Scanning Probe Microscopy and Spectroscopy: Methods and Applications. 1994.

[196] Joachim Loos. "The art of SPM: Scanning probe microscopy in materials science". In: Advanced Materials 17 (15) (2005), pp. 1821-1833. DOI: 10 . 1002 /adma. 200500701.

[197] R. Garcia, A. W. Knoll, and E. Riedo. "Advanced Scanning Probe Lithography." In: Nature Nanotechnology 9 (8) (2014), p. 577. DOI: 10.1038 / nnano . 2014.157.

[198] J. P. Rabe and S. Bucholz. "Commensurability and Mobility in TwoDimensional Molecular Patterns on Graphite". In: Science 253 (5018) (1991), pp. 424-427.

[199] E. Laegsgaard, L. Osterlund, P. Thostrup, P. B. Rasmussen, I. Stensgaard, and F. Besenbacher. "A high-pressure scanning tunneling microscope". In: Review of Scientific Instruments 72 (9) (2001), pp. 3537-3542. DOI: 10.1063 / 1.1389497.

[200] J. A. Dagata, J. Schneir, H. H. Harary, C. J. Evans, M. T. Postek, and J. Bennett. "Modification of hydrogenpassivated silicon by a scanning tunneling microscope operating in air". In: Applied Physics Letters 56 (20) (1990). DOI: http://dx.doi.org/10.1063/1.102999.

[201] R. J. Hamers, R. M. Tromp, and J. E. Demuth. "Surface Electronic Structure of Si (111)-(7x7) Resolved in Real Space". In: Physical Review Letters 56 (18) (1986), pp. 1972-1975. DOI: 10.1103/PhysRevLett.56.1972.

[202] O. Fischer, M. Kugler, I. Maggio-Aprile, C. Berthod, and C. Renner. "Scanning tunneling spectroscopy of high-temperature superconductors". In: Reviews of Modern Physics 79 (1) (2007), pp. 353-419.

[203] G. Meyer and N. M. Amer. "Simultaneous measurement of lateral and normal forces with an opticalbeamdeflection atomic force microscope". In: Applied Physics Letters 57 (20) (1990). DOI: $10.1063 / 1.102985$.

[204] S. Alexander, L. Hellemans, O. Marti, J. Schneir, V. Elings, P. K. Hansma, M. Longmire, and J. Gurley. "An atomicresolution atomicforce microscope implemented using an optical lever". In: Journal of Applied Physics 65 (1) (1989).

[205] G. Meyer and N. M. Amer. "Opticalbeamdeflection atomic force microscopy: The $\mathrm{NaCl}$ (001) surface". In: Applied Physics Letters 56 (21) (1990).

[206] B. Voigtlander. Scanning Probe Microscopy. 2015, p. 375. DOI: $10.1007 /$ 978-3-662-45240-0.

[207] R. Garcia and R. Perez. "Dynamic atomic force microscopy methods". In: Surface Science Reports 47 (6-8) (2002), pp. 197-301. DOI: 10.1016 / S 0167 5729(02) $00077-8$.

[208] R. Garcia, R. V Martinez, and J. Martinez. "Nano-chemistry and scanning probe nanolithographies". In: Chemical Society Reviews 35 (1) (2005), pp. 2938. DOI: $10.1039 /$ b501599p.

[209] R. Garcia, M. Calleja, and H. Rohrer. "Patterning of silicon surfaces with noncontact atomic force microscopy: Field-induced formation of nanometer-size water bridges". In: Journal of Applied Physics 86 (4) (1999), pp. 1898-1903. DOI: $10.1063 / 1.370985$. 
[210] S. Kalinin. Scanning Probe Microscopy Electrical and Electromechanical Phenomena at the Nanoscale. Vol. II. 4. Springer, 2007, p. 980. DOI: $10.1007 / 978-$ $0-387-28668-6$.

[211] S. V. Kalinin, A. N. Morozovska, L. Q. Chen, and B. J. Rodriguez. "Local polarization dynamics in ferroelectric materials". In: Reports on Progress in Physics 73 (5) (2010), p. 056502. DOI: $10.1088 / 0034-4885 / 73 / 5 /$ 056502 .

[212] I. Gaponenko, P. Tückmantel, J. Karthik, L. W. Martin, and P. Paruch. “Towards reversible control of domain wall conduction in $\mathrm{PbZr}_{0.2} \mathrm{Ti}_{0.8} \mathrm{O}_{3}$ thin films". In: Applied Physics Letters 106 (16) (2015), pp. 0-4. DOI: $10.1063 / 1$. 4918762.

[213] N. Domingo, L. Lopez-Mir, M. Paradinas, V. Holy, J. Zelezny, D. Yi, S. J. Suresha, J. Liu, C. Rayan Serrao, R. Ramesh, C. Ocal, X. Marti, and G. Catalan. "Giant reversible nanoscale piezoresistance at room temperature in $\mathrm{Sr}_{2} \mathrm{IrO}_{4}$ thin films". In: Nanoscale 7 (8) (2015), pp. 3453-3459. DOI: 10 . 1039/C4NR06954D.

[214] T. Jia, H. Kimura, Z. Cheng, and H. Zhao. "Switching of both local ferroelectric and magnetic domains in multiferroic $\mathrm{Bi}_{0.9} \mathrm{La}_{0.1} \mathrm{FeO}_{3}$ thin film by mechanical force". In: Scientific Reports 6 (August) (2016), p. 31867. DOI: $10.1038 /$ srep31867.

[215] Ph Avouris, R. Marte, T. Hertel, and R. Sandstrom. "AFM-tip-induced and current-induced local oxidation of silicon andmetals". In: Applied Physics A: Materials Science and Processing 66 (1998). DOI: 10.1007 /s 003390051218.

[216] S. Rozhok, P. Sun, R. Piner, M. Lieberman, and C. A. Mirkin. “AFM study of water meniscus formation between an AFM tip and $\mathrm{NaCl}$ substrate". In: Journal of Physical Chemistry B 108 (23) (2004), pp. 7814-7819. DOI: 10.1021 / jp0401269.

[217] Lawrence D. Fitch and Vernon L. Burdick. "Water corrosion of $\mathrm{YBa}_{2} \mathrm{Cu}_{3} \mathrm{O}_{7}$ superconductors". In: Journal of the American Ceramic Society 72 (10) (1989), pp. 2020-2023.

[218] R. Zhao and S. Myhra. "Environmental degradation of $\mathrm{YBa}_{2} \mathrm{Cu}_{3} \mathrm{O}_{7-x}$ : Adescriptiveandpredictivemodel". In: Physica C 230 (1-2) (1994), pp. 75-81.

[219] P. Staszczuk, G. W. Ch, and D. Sternik. "Thermogravimetric and microgravimetric studies of the surface properties of a high-temperature superconductor". In: Journal of thermal analysis and calorimetry 62 (2000), pp. 451-459.

[220] S. Q. Liu, N. J. Wu, and A. Ignatiev. "Electric-pulse-induced reversible resistance change effect in magnetoresistive films". In: Applied Physics Letters 76 (2000) (2000), p. 2749. DOI: $10.1063 / 1.126464$.

[221] aA. Baikalov, Y. Q. Wang, B. Shen, B. Lorenz, S. Tsui, Y. Y. Sun, Y. Y. Xue, and $\mathrm{C} . \mathrm{W}$. Chu. "Field-driven hysteretic and reversible resistive switch at the Ag- $\mathrm{Pr}_{0.7} \mathrm{Ca}_{0.7} \mathrm{MnO}_{3}$ interface". In: Applied Physics Letters 83 (5) (2003), p. 957. DOI: $10.1063 / 1.1590741$.

[222] A. Sawa, T. Fujii, M. Kawasaki, and Y. Tokura. "Hysteretic current-voltage characteristics and resistance switching at a rectifying $\mathrm{Ti} / \mathrm{Pr}_{0.7} \mathrm{Ca}_{0.3} \mathrm{MnO}_{3}$ interface". In: Applied Physics Letters 85 (18) (2004), pp. 4073-4075. DOI: $10.1063 / 1.1812580$.

[223] A. Odagawa, T. Kanno, and H. Adachi. "Transient response during resistance switching in $\mathrm{AgPr}_{0.7} \mathrm{Ca}_{0.3} \mathrm{MnO}_{3} \mathrm{Pt}$ thin films". In: Journal of Applied Physics 99 (1) (2006), p. 016101.

[224] Y. B. Nian, J. Strozier, N. J. Wu, X. Chen, and A. Ignatiev. "Evidence for an oxygen diffusion model for the electric pulse induced resistance change 
effect in transition-metal oxides". In: Physical Review Letters 98 (14) (2007), pp. 3-6. DOI: 10.1103/PhysRevLett.98.146403.

[225] F. Borgatti, C. Park, A. Herpers, F. Offi, R. Egoavil, Y. Yamashita, A. Yang, M. Kobata, K. Kobayashi, J. Verbeeck, G. Panaccione, and R. Dittmann. "Chemical insight into electroforming of resistive switching manganite heterostructures." In: Nanoscale 5 (9) (2013), pp. 3954-60. DOI: 10.1039 / c3nr00106g.

[226] J.-O. Krisponeit, C. Kalkert, B. Damaschke, V. Moshnyaga, and K. Samwer. "Nanoscale resistance switching in manganite thin films: Sharp voltage threshold and pulse-width dependence". In: Physical Review B 82 (14) (2010), p. 144440. DOI: 10.1103 /PhysRevB.82.144440.

[227] N. Ghenzi, M. J. Sanchez, F. Gomez-Marlasca, P. Levy, and M. J. Rozenberg. "Hysteresis switching loops in Ag-manganite memristive interfaces". In: Journal of Applied Physics 107 (9) (2010). DOI: $10.1063 / 1.3372617$.

[228] J.-O. Krisponeit, C. Kalkert, B. Damaschke, V. Moshnyaga, and K. Samwer. "Time-resolved resistive switching on manganite surfaces: Creep and $1 / \mathrm{f}_{\alpha}$ noise signatures indicate pinning of nanoscale domains". In: Physical Review B 87 (12) (2013), p. 121103. DOI: 10.1103/PhysRevB.87.121103.

[229] X. Chen, N. Wu, J. Strozier, and A. Ignatiev. "Spatially extended nature of resistive switching in perovskite oxide thin films". In: Applied Physics Letters 89 (6) (2006), p. 063507. DOI: $10.1063 / 1.2236213$.

[230] H. K. Lau and C. W. Leung. "Nonvolatile multilevel memory effect by resistive switching in manganite thin films". In: Journal of Applied Physics 104 (12) (2008). DOI: $10.1063 / 1.3043801$.

[231] D. Liu, N. Wang, G. Wang, Z. Shao, X. Zhu, C. Zhang, and H. Cheng. "Nonvolatile bipolar resistive switching in amorphous Sr-doped $\mathrm{LaMnO}_{3}$ thin films deposited by radio frequency magnetron sputtering". In: Applied Physics Letters 102 (2013), p. 134105. DOI: 10.1063/1.4800229.

[232] Y. W. Xie, J. R. Sun, D. J. Wang, S. Liang, and B. G. Shen. "Reversible electroresistance at the $\mathrm{Ag} / \mathrm{La}_{0.67} \mathrm{Sr}_{0.33} \mathrm{MnO}_{3}$ interface". In: Journal of Applied Physics 100 (3) (2006), pp. 1-6. DOI: $10.1063 / 1.2222069$.

[233] X.D. Luo, R.L. Gao, C.L. Fu, W. Cai, G. Chen, X.L. Deng, H.R Zhang, and J.R. Sun. "Resistance switching mechanism of $\mathrm{La}_{0.8} \mathrm{Sr}_{0.2} \mathrm{MnO}_{3-\delta}$ thin films". In: Physica B: Condensed Matter 483 (2016), pp. 99-102. DOI: $10.1016 / j$. physb.2015.12.031.

[234] R. Munstermann, J. J. Yang, J. P. Strachan, G. Medeiros-Ribeiro, R. Dittmann, and R. Waser. "Morphological and electrical changes in $\mathrm{TiO}_{2}$ memristive devices induced by electroforming and switching". In: physica status solidi (RRL) - Rapid Research Letters 4 (1-2) (2010), pp. 16-18. DOI: 10 . 1002 / pssr.200903347.

[235] J. Y. Son and Y. H. Shin. "Direct observation of conducting filaments on resistive switching of $\mathrm{NiO}$ thin films". In: Applied Physics Letters 92 (22) (2008), pp. 10-13. DOI: $10.1063 / 1.2931087$.

[236] F. Nardi, D. Deleruyelle, S. Spiga, C. Muller, B. Bouteille, and D. Ielmini. "Switching of nanosized filaments in $\mathrm{NiO}$ by conductive atomic force microscopy". In: Journal of Applied Physics 112 (6) (2012), pp. 0-6. DOI: 10 . $1063 / 1.4752032$.

[237] Z. Xu, K. Jin, L. Gu, Y. Jin, C. Ge, C. Wang, H. Guo, H. Lu, R. Zhao, and G. Yang. "Evidence for a crucial role played by oxygen vacancies in $\mathrm{LaMnO}_{3}$ resistive switching memories." In: Small 8 (8) (2012), pp. 1279-84. DOI: 10 . $1002 / \operatorname{smll} .201101796$. 
[238] L. Yao, S. Inkinen, and S. van Dijken. "Direct observation of oxygen vacancydriven structural and resistive phase transitions in $\mathrm{La}_{2 / 3} \mathrm{Sr}_{1 / 3} \mathrm{MnO}_{3}$ ". In: Nature Communications 8 (2017), p. 14544. DOI: 10.1038 /ncomms 14544.

[239] J. Kim, C. Ko, A. Frenzel, S. Ramanathan, and J. E. Hoffman. "Nanoscale imaging and control of resistance switching in $\mathrm{VO}_{2}$ at room temperature". In: Applied Physics Letters 96 (21) (2010), pp. 10-13. DOI: 10 . $1063 / 1$. 3435466.

[240] M. Scherff, J. Hoffmann, B. Meyer, T. Danz, and C. Jooss. "Interplay of crossplane polaronic transport and resistive switching in $\mathrm{Pt}_{-} \mathrm{Pr}_{0.67} \mathrm{Ca}_{0.33} \mathrm{MnO}_{3}-\mathrm{Pt}$ heterostructures". In: New Journal of Physics 15 (2013). DOI: 10 . $1088 /$ $1367-2630 / 15 / 10 / 103008$.

[241] J. Song and J. Lee. "Self-assembled nanostructured resistive switching memory devices fabricated by templated bottom-up growth". In: Scientific Reports (2016), pp. 1-7. DOI: $10.1038 /$ srep18967.

[242] K. Min Kim, B. J. Choi, B. W. Koo, S. Choi, D. S. Jeong, and C. S. Hwang. "Resistive switching in $\mathrm{PtAl}_{2} \mathrm{O}_{3} \mathrm{TiO}_{2} \mathrm{Ru}$ stacked structures". In: Electrochemical and Solid-State Letters 9 (12) (2006), G343-G346.

[243] S. O. Kang, I. Hwang, J. Choi, S. Hong, J. S. Kim, I. S. Byun, J. H. Bahng, J. Y. Koo, and B. H. Park. "Direct investigation on conducting nanofilaments in single-crystalline $\mathrm{Ni} / \mathrm{NiO}$ core/shell nanodisk arrays". In: Applied Physics Letters 96 (5) (2010), pp. 10-13. DOI: $10.1063 / 1.3301620$.

[244] C. Yoshida, K. Kinoshita, T. Yamasaki, and Y. Sugiyama. "Direct observation of oxygen movement during resistance switching in $\mathrm{NiO} / \mathrm{Pt}$ film". In: $\mathrm{Ap}$ plied Physics Letters 93 (4) (2008), pp. 2006-2009. DOI: $10.1063 / 1.2966141$.

[245] M. H. Lee, S. J. Song, K. M. Kim, G. H. Kim, J. Y. Seok, J. H. Yoon, and C. S. Hwang. "Scanning probe based observation of bipolar resistive switching NiO films". In: Applied Physics Letters 97 (6) (2010), pp. 107-110. DOI: 10 . $1063 / 1.3479526$.

[246] H. Shima, F. Takano, H. Muramatsu, M. Yamazaki, H. Akinaga, and A. Kogure. "Local chemical state change in $\mathrm{Co}-\mathrm{O}$ resistance random access memory". In: physica status solidi (RRL) - Rapid Research Letters 2 (3) (2008), pp. 99-101. DOI: 10.1002/pssr.200802003.

[247] Min Hwan Lee and Cheol Seong Hwang. "Resistive switching memory: observations with scanning probe microscopy." In: Nanoscale 3 (2) (2011), pp. 490-502. DOI: $10.1039 / \mathrm{c} 0 \mathrm{nr} 00580 \mathrm{k}$.

[248] R. Ortega-Hernandez, M. Coll, J. Gonzalez-Rosillo, A. Palau, X. Obradors, E. Miranda, T. Puig, and J. Sune. "Resistive switching in $\mathrm{CeO}_{2} / \mathrm{La}_{0.8} \mathrm{Sr}_{0.2} \mathrm{MnO}_{3}$ bilayer for non-volatile memory applications". In: Microelectronic Engineering 147 (2015), pp. 37-40. DOI: $10.1016 /$ j.mee.2015.04.042.

[249] C. H. Ahn, J.-M. Triscone, and J. Mannhart. "Electric field effect in correlated oxide systems." In: Nature 424 (6952) (2003), pp. 1015-8. DOI: $10.1038 /$ nature 01878.

[250] T. K. Ghanem, E. D. Williams, and M. S. Fuhrer. "Characterization of the electrical contact between a conductive atomic force microscope cantilever and a carbon nanotube". In: Journal of Applied Physics 110 (5) (2011), pp. 1-7. DOI: $10.1063 / 1.3626811$.

[251] J. Alvarez, I. Ngo, M.-E. Gueunier-Farret, J.-. Kleider, L. Yu, P. R. Cabarrocas, S. Perraud, E. Rouviere, C. Celle, C. Mouchet, and J.-P. Simonato. "Conductive-probe atomic force microscopy characterization of silicon nanowire." In: Nanoscale research letters 6 (1) (2011), p. 110. DOI: $10.1186 /$ 1556-276X-6-110. 
[252] K H Ahn, T Lookman, and A R Bishop. "Strain-induced metal-insulator phase coexistence in perovskite manganites". In: Nature 428 (2004) (2004), pp. 401-404. DOI: 10.1038/nature02411.1..

[253] S. V. Trukhanov, L. S. Lobanovski, M. V. Bushinsky, V. a. Khomchenko, N. V. Pushkarev, I. O. Troyanchuk, a. Maignan, D. Flahaut, H. Szymczak, and R. Szymczak. "Influence of oxygen vacancies on the magnetic and electrical properties of $\mathrm{La}_{1-x} \mathrm{Sr}_{x} \mathrm{MnO}_{3-x / 2}$ manganites". In: The European Physical Journal B - Condensed Matter and Complex Systems 42 (2004), pp. 51-61. DOI: $10.1140 /$ epjb/e2004-00357-8.

[254] T. Becker, C. Streng, Y. Luo, V. Moshnyaga, B. Damaschke, N. Shannon, and K. Samwer. "Intrinsic inhomogeneities in manganite thin films investigated with Scanning Tunneling Spectroscopy". In: Physical Review Letters 89 (23) (2002), p. 237203. DOI: 10.1103/PhysRevLett.89.237203.

[255] C. Renner, G. Aeppli, B.-G. Kim, Y.-A. Soh, and S.-W. Cheong. "Atomicscale images of charge ordering in a mixed-valence manganite." In: Nature 416 (6880) (2002), pp. 518-21. DOI: $10.1038 / 416518$ a.

[256] Y. Xu, K. Steenbeck, P. Das, J. Wei, M. Koblischka, and U. Hartmann. "Local electronic properties of manganite films studied by STM/STS under ambient conditions". In: Journal of Magnetism and Magnetic Materials 272-276 (2004), pp. 875-876. DOI: $10.1016 / j$.jmmm.2003.12.196.

[257] R. Di Capua, C. A. Perroni, V. Cataudella, F. Miletto Granozio, P. Perna, M. Salluzzo, U. Scotti di Uccio, and R. Vaglio. "Direct observation of spectroscopic inhomogeneities on $\mathrm{La}_{0.7} \mathrm{Sr}_{0.3} \mathrm{MnO}_{3}$ thin films by scanning tunnelling spectroscopy". In: Journal of Physics: Condensed Matter 18 (35) (2006), pp. 8195-8204. DOI: $10.1088 / 0953-8984 / 18 / 35 / 007$.

[258] S. Seiro, Y. Fasano, I. Maggio-Aprile, O. Kuffer, and O. Fischer. "Homogeneous spectroscopic properties in manganite films". In: Journal of Magnetism and Magnetic Materials 310 (2) (2007), pp. 243-245. DOI: 10 .1016/ j. jmmm. 2006.10 .1088$.

[259] J. X. Ma, D. T. Gillaspie, E. W. Plummer, and J. Shen. "Visualization of localized holes in manganite thin films with atomic resolution". In: Physical Review Letters 95 (23) (2005), pp. 1-4. DOI: 10.1103 / PhysRevLett. 95. 237210.

[260] H. M. Ronnow, C. Renner, G. Aeppli, T. Kimura, and Y. Tokura. "Polarons and confinement of electronic motion to two dimensions in a layered manganite." In: Nature 440 (7087) (2006), pp. 1025-1028. DOI: 10 . 1038 / nature 04650.

[261] U. R. Singh, A. K. Gupta, G. Sheet, V. Chandrasekhar, H. W. Jang, and C. B. Eom. "Pseudogap formation in the metallic state of $\mathrm{La}_{0.7} \mathrm{Sr}_{0.3} \mathrm{MnO}_{3}$ thinfilms". In: Applied Physics Letters 93 (21) (2008), p. 212503.

[262] S. Seiro, Y. Fasano, I. Maggio-Aprile, E. Koller, O. Kuffer, and O. Fischer. "Polaronic signature in the metallic phase of $\mathrm{La}_{0.7} \mathrm{Ca}_{0 . e} \mathrm{MnO}_{3}$ films detected by scanning tunneling spectroscopy". In: Physical Review B 77 (2) (2008), p. 020407. DOI: 10.1103/PhysRevB.77.020407.

[263] S. Seiro, Y. Fasano, I. Maggio-Aprile, E. Koller, R. Lortz, and O. Fischer. “Fingerprint of dynamical spin-charge correlations in the tunneling spectra of colossal magnetoresistive manganites". In: Physical Review B 80 (10) (2009), p. 100404. DOI: $10.1103 /$ PhysRevB.80.100404.

[264] M. Moors, K. K. Adepalli, Q. Lu, A. Wedig, C. Baumer, K. Skaja, B. Arndt, H. L. Tuller, R. Dittmann, R. Waser, B. Yildiz, and I. Valov. "Resistive switching 
mechanisms on $\mathrm{TaO}_{x}$ and $\mathrm{SrRuO}_{3}$ thin-film surfaces probed by scanning tunneling microscopy". In: ACS Nano 10 (2016), pp. 1481 -1492. DOI: 10 . 1021 /acsnano.5b07020.

[265] Y. L. Chen, J. Wang, C. M. Xiong, R. F. Dou, J. Y. Yang, and J. C. Nie. “Scanning tunneling microscopy/spectroscopy studies of resistive switching in $\mathrm{Nb}$-doped $\mathrm{SrTiO}_{3}{ }^{\prime \prime}$. In: Journal of Applied Physics 112 (2) (2012), p. 023703. DOI: $10.1063 / 1.4733999$.

[266] A. Wedig, M. Luebben, M. Moors, D. Y. Cho, K. Skaja, T. Hasegawa, K. K. Adepalli, B. Yildiz, R. Waser, and I. Valov. "Nanoscale cation motion in $\mathrm{TaO}_{x}, \mathrm{HfO}_{x}$ and $\mathrm{TiO}_{x}$ memristive systems". In: Nature Nanotechnology 11 (1) (2015), pp.67-74. DOI: $10.1038 /$ nnano.2015.221.

[267] E. L. Wolf. Principles of Electron Tunneling Spectroscopy: Second Edition. 2nd ed. Oxford University Press, 2011, p. 616. DOI: $10.1093 /$ acprof : oso/ 9780199589494.001 .0001$.

[268] A. Biswas, S. Elizabeth, A. K. Raychaudhuri, and H. L. Bhat. “Density of states of hole-doped manganites: A scanning-tunneling-microscopy / spectroscopy study". In: Physical Review B 59 (8) (1999), pp. 5368-5376. DOI: https://doi.org/10.1103/PhysRevB.59.5368.

[269] Lina Chen. "Microscopic study of structure, chemical composition and local conductivity of $\mathrm{La}_{2 / 3} \mathrm{Sr}_{1 / 3} \mathrm{MnO}_{3}$ films". PhD thesis. Louisiana State University, 2016.

[270] D. S. Jeong, H. Schroeder, U. Breuer, and R. Waser. "Characteristic electroforming behavior in $\mathrm{Pt} / \mathrm{TiO}_{2} / \mathrm{Pt}$ resistive switching cells depending on atmosphere". In: Journal of Applied Physics 104 (12) (2008). DOI: 10.1063 / 1.3043879.

[271] L. Goux, P. Czarnecki, Y. Y. Chen, L. Pantisano, X. P. Wang, R. Degraeve, B. Govoreanu, M. Jurczak, D. J. Wouters, and L. Altimime. "Evidences of oxygen-mediated resistive-switching mechanism in TiN/ $\mathrm{HfO}_{2} / \mathrm{Pt}$ cells". In: Applied Physics Letters 97 (24) (2010), pp. 24-27. DOI: $10.1063 / 1$. 3527086.

[272] Jr Jian Ke, Zi Jheng Liu, Chen Fang Kang, Su Jien Lin, and Jr Hau He. "Surface effect on resistive switching behaviors of $\mathrm{ZnO}^{\prime}$. In: Applied Physics Letters 99 (19) (2011), pp. 103-106.

[273] J. P. Strachan, J. J. Yang, R. Muenstermann, A. Scholl, G. Medeiros-Ribeiro, D. R. Stewart, and R. Stanley Williams. "Structural and chemical characterization of $\mathrm{TiO}_{2}$ memristive devices by spatially-resolved NEXAFS." In: Nanotechnology 20 (48) (2009), p. 485701. DOI: 10 .1088/0957-4484/20/ $48 / 485701$.

[274] R. Yang, K. Terabe, T. Tsuruoka, T. Hasegawa, and M. Aono. “Oxygen migration process in the interfaces during bipolar resistance switching behavior of $\mathrm{WO}_{3-x}-$ basednanoionicsdevices". In: Applied Physics Letters 100 (23) (2012), pp. 1-5. DOI: $10.1063 / 1.4726084$.

[275] M. Shirpour, G. Gregori, R. Merkle, and J. Maier. "On the proton conductivity in pure and gadolinium doped nanocrystalline cerium oxide". In: Physical Chemistry Chemical Physics 13 (3) (2011), pp. 937-940. DOI: $10.1039 /$ c0 $0 \mathrm{cp} 01702 \mathrm{~g}$.

[276] A. Feng, F. El Gabaly, X. Ye, Z.-X. Shen, and W. C. Chueh. "Fast vacancymediated oxygen ion incorporation across the ceria-gas electrochemical interface". In: Nature Communications 5 (2014), p. 4374. DOI: $10.1038 /$ ncomms 5374. 
[277] S. Tappertzhofen, I. Valov, T. Tsuruoka, T. Hasegawa, R. Waser, and M. Aono. "Generic Relevance of Counter Charges for Cation-Based Nanoscale Resistive Switching Memories". In: ACS Nano 7 (7) (2013), pp. 6396-6402.

[278] R. Merkle and J. Maier. "How is oxygen incorporated into oxides? A comprehensive kinetic study of a simple solid-state reaction with $\mathrm{SrTiO}_{3}$ as a model material". In: Angewandte Chemie 47 (21) (2008), pp. 3874-3894. DOI: 10.1002 /anie.200700987.

[279] Y. A. Mastrikov, R. Merkle, E. Heifets, E. A. Kotomin, and J. Maier. "Pathways for oxygen incorporation in mixed conducting perovskites: A DFTbased Mechanistic analysis for (La,Sr) $\mathrm{MnO}_{3-x}$ ". In: Journal of Physical Chemistry C 114 (7) (2010), pp. 3017-3027. DOI: 10.1021 / jp 909401 g.

[280] S.C. Singhal. "Solid oxide fuel cells". In: The Electrochemical Society Interface (2007), pp. 41-44. DOI: $10.1039 /$ b105764m.

[281] M. M. Kuklja, E. a Kotomin, R. Merkle, Y. a Mastrikov, and J. Maier. “Combined theoretical and experimental analysis of processes determining cathode performance in solid oxide fuel cells." In: Physical chemistry chemical physics 15 (15) (2013), pp. 5443-5471. DOI: 10.1039 / c3cp 44363 a.

[282] V. Alexandrov, S. Piskunov, Y. F. Zhukovskii, E. A Kotomin, and J. Maier. "First-principles modeling of oxygen interaction with $\mathrm{SrTiO}_{3}(001)$ surface: Comparative density-functional LCAO and plane-wave study". In: Integrated Ferroelectrics 123 (2011), pp. 10-17. DOI: $10.1080 / 10584587.2011$. 570586.

[283] J. Fleig, R. Merkle, and J. Maier. "The $\mathrm{p}\left(\mathrm{O}_{2}\right)$ dependence of oxygen surface coverage and exchange current density of mixed conducting oxide electrodes: model considerations." In: Physical chemistry chemical physics 9 (21) (2007), pp. 2713-2723. DOI: $10.1039 /$ b618765j.

[284] A. Plecenik, M. Grajcar, P. Seidel, S. Takacs, A. Matthes, M. Zuzcak, and S. Beňacvka. "Influence of bias voltage history on conductance properties of YBaCuO/normal metal junctions". In: Physica C: Superconductivity 301 (3-4) (1998), pp. 234-242. DOI: 10.1016/S0921-4534(98)00116-6.

[285] H. J. Zhang, X. P. Zhang, J. P. Shi, H. F. Tian, and Y. G. Zhao. "Effect of oxygen content and superconductivity on the nonvolatile resistive switching in $\mathrm{YBa}_{2} \mathrm{Cu}_{3} \mathrm{O}_{6+x} / \mathrm{Nb}$-doped $\mathrm{SrTiO}_{3}$ heterojunctions". In: Applied Physics Letters 94 (9) (2009), p. 092111. DOI: $10.1063 / 1.3095493$.

[286] C. Acha and M. J. Rozenberg. "Non-volatile resistive switching in the dielectric superconductor $\mathrm{YBa}_{2} \mathrm{Cu}_{3} \mathrm{O}_{7-\delta}$ ". In: Journal of physics. Condensed matter 21 (4) (2009), p. 045702. DOI: 10 . 1088/0953-8984/21/4/045702.

[287] C. Acha. "Electric pulse-induced resistive switching in ceramic interfaces". In: Physica B: Condensed Matter 404 (18) (2009), pp. 2746-2748. DOI: 10 . 1016/j.physb.2009.06.111.

[288] A. Plecenik, M. Tomasek, T. Plecenik, M. Truchly, J. Noskovic, M. Zahoran, T. Roch, M. Belogolovskii, M. Spankova, S. Chromik, and P. Kus. "Studies of resistance switching effects in metal/ $\mathrm{YBa}_{2} \mathrm{Cu}_{3} \mathrm{O}_{7-x}$ interface junctions". In: Applied Surface Science 256 (18) (2010), pp. 5684-5687. DOI: 10.1016 / j. apsusc.2010.03.018.

[289] C. Acha. "Dynamical behaviour of the resistive switching in ceramic YBCO/metal interfaces". In: Journal of Physics D: Applied Physics 44 (34) (2011), p. 345301. DOI: $10.1088 / 0022-3727 / 44 / 34 / 345301$.

[290] M. Tomasek, T. Plecenik, M. Truchly, J. Noskovic, T. Roch, M. Zahoran, S. Chromik, M. Spankova, P. Kus, and A Plecenik. "Temperature dependence of the resistance switching effect studied on the metal/ $\mathrm{YBa}_{2} \mathrm{Cu}_{3} \mathrm{O}_{6+x}$ planar 
junctions". In: Journal of Vacuum Science \& Technology B: Microelectronics and Nanometer Structures 29 (1) (2011), 01AD04. DOI: $10.1116 / 1.3521408$.

[291] A. Schulman and C. Acha. "Resistive switching effects on the spatial distribution of phases in metal-complex oxide interfaces". In: Physica B: Condensed Matter 407 (16) (2012), pp. 3147-3149. DOI: 10 . $1016 /$ j . physb . 2011.12 .049$.

[292] B. O'Reagan and M. Gratzel. "Screw dislocations in high-Tc films". In: Nature 350 (1991), pp. 279-280.

[293] M. E. Hawley, Ian D. Raistrick, Jerome G. Beery, and Robert J. Houlton. "Growth mechanism of sputtered films of $\mathrm{YBa}_{2} \mathrm{Cu}_{3} \mathrm{O}_{7}$ Studied by scanning tunneling microscopy". In: Science 251 (1991), pp. 1587-1589.

[294] J. Gazquez, M. Coll, N. Roma, F. Sandiumenge, T. Puig, and X. Obradors. "Structural defects in trifluoroacetate derived $\mathrm{YBa}_{2} \mathrm{Cu}_{3} \mathrm{O}_{7}$ thin films". In: Superconductor Science \& Technology 25 (6) (2012), p. 65009.

[295] H. Castro and G. Deutscher. "Anomalous Fermi liquid behavior of overdoped high-Tc superconductors". In: Physical Review B - Condensed Matter and Materials Physics 70 (17) (2004), pp. 1-8. DOI: 10.1103 / PhysRevB . 70.174511.

[296] A. Palau, C. Monton, V. Rouco, X. Obradors, and T. Puig. "Guided vortex motion in $\mathrm{YBa}_{2} \mathrm{Cu}_{3} \mathrm{O}_{7}$ thin films with collective ratchet pinning potentials". In: Physical Review B 85 (1) (2012), pp. 1-5. DOI: 10.1103 / PhysRevB . 85 . 012502.

[297] R. Scherwitzl, P. Zubko, C. Lichtensteiger, and J. M. Triscone. "Electric-field tuning of the metal-insulator transition in ultrathin films of $\mathrm{LaNiO}_{3}{ }^{\prime \prime}$. In: Applied Physics Letters 95 (22) (2009), pp. 1-4. DOI: $10.1063 / 1.3269591$.

[298] J. Shi, S. D. Ha, Y. Zhou, F. Schoofs, and S. Ramanathan. "A correlated nickelate synaptic transistor". In: Nature Communications 4 (Oct. 2013), p. 2676. DOI: $10.1038 /$ ncomms 3676 .

[299] J. Shi, Y. Zhou, S. Ramanathan, and J. Shi. "Colossal resistance switching and band gap modulation in a perovskite nickelate by electron doping." In: Nature communications 5 (2014), p. 4860. DOI: 10.1038 / ncomms 5860.

[300] James M. Rondinelli, Steven J. May, and John W. Freeland. "Control of octahedral connectivity in perovskite oxide heterostructures: An emerging route to multifunctional materials discovery". In: MRS Bulletin 37 (03) (2012), pp. 261-270. DOI: $10.1557 / \mathrm{mrs} .2012 .49$. 